\title{
Analysis of Radiographic Contrast Markers for X-ray Digital Image Correlation of Tissue-Simulants under Dynamic Load
}

\author{
by \\ Stéphane Magnan
}

A thesis submitted to the Faculty of Graduate Studies and Research in partial fulfilment of the requirements for the degree of

\author{
Master of Applied Science \\ in \\ Mechanical Engineering
}

Ottawa-Carleton Institute for Mechanical and Aerospace Engineering

Department of Mechanical and Aerospace Engineering

Carleton University

Ottawa, Ontario, Canada

August 2018

Copyright $(\subset$

2018 - Stéphane Magnan 
The undersigned recommend to the Faculty of Graduate Studies and Research acceptance of the Thesis

\title{
Analysis of Radiographic Contrast Markers for X-ray Digital Image Correlation of Tissue-Simulants under Dynamic Load
}

\author{
Submitted by Stéphane Magnan \\ in partial fulfilment of the requirements for the degree of \\ Master of Applied Science
}

O.E. Petel, Thesis Supervisor

J. Laliberte, M\&AE

J. Green, SYSC

A. Weck, UOttawa

R. Miller, Department Chair

Carleton University

2018 


\section{Abstract}

The study of traumatic brain injury is critical to the improvement of protective equipment. Numerical models of brain deformation require real-world data for validation. In preparation for upcoming cadaver studies, a novel method of measuring displacement and strain fields of optically inaccessible internal planes using high-speed X-ray, embedded contrast markers and digital image correlation (DIC) is presented herein. An uncoupled scintillator and optimally-selected highspeed camera enable continuous X-ray imaging through a human head at 10,000 fps. As varying composition creates radiographic contrast, contrast within a human brain is limited, therefore, artificial contrast markers are required. Markers must be dynamically coupled to the bulk material and provide sufficient $X$-ray contrast. An analytical tool was developed to design of contrast markers. The impact of contrast-to-noise ratio and out-of-plane motion on DIC accuracy were quantified. Finally, a feasibility study using a biofidelic headform subjected to a NOCSAE drop test is presented. 


\section{Acknowledgments}

As I turn the page on this chapter of my life, I cannot help but to reflect on the enormous contributions of an incredible team of collaborators that made it all possible. The completion of this work is due in no small part to my advisor, Professor Oren Petel. His support and passion for my research, made this experience enjoyable. You have provided me with unique opportunities to explore my field over the last two years, encouraging personal growth, and leading me to ask the important question. I am sincerely grateful for your insight and guidance in this work.

Construction of the high-speed X-ray facility would not have been possible without the technical recommendations of support staff in the department of mechanical and aerospace engineering at Carleton University. Specifically, the expertise and guidance of Alex Proctor, Ian Lloyd, Kevin Sangster, and Stephan Bilijan, contributed enormously to this project.

Preliminary testing would not have been possible without Professors Paul Johns and Tong $\mathrm{Xu}$, of the Department of Physics at Carleton University, for allowing access to their X-ray system until our facility was operational. Tong, your passion for discussions on X-ray physics helped establish the foundation I required for my research.

Unique opportunities to explore other research facilities have complemented my studies and contributed to my personal growth as a researcher. Special thanks 
to Simon Ouellet, of Defence Research and Development Canada (DRDC) Valcatier, for access to the $\mathrm{BI}^{2} \mathrm{PED}$ headform, and discussion of radiographic contrast marker integration; and Rohan Banton and Thuvan Piehler of the U.S. Army Research Laboratory, for headforms and brain surrogate materials.

The first people to share in my successes and support my failures were often my coworkers. Specifically, shout-outs to Ashley Mazurkiewicz, for always listening to the latest updates and sharing stellar LOTR memes as encouragement, and to Scott Dutrisac for solving so many of my problems, and helping me with more rebuilds than the Edmonton Oilers.

My journey over the past two years would not have been possible without the unwavering support of those closest to me. To Mom and Dad, I would not be where I am today without your constant belief that I would always eventually succeed and overcome the countless setbacks along the way. To my sister Chantal, your excitement and pride in my work always kept me going, encouraging me to face challenges head-on. To my fiancée Stéphanie, you've been there for me every step of the way, pushing me to realize my full potential while putting up with long hours and weeks of "just one chapter left in my thesis".

Merci à tous. 


\section{Table of Contents}

Abstract $\quad$ iii

Acknowledgments $\quad$ iv

Table of Contents vi vi

List of Tables $\quad$ x

List of Figures $\quad$ xi

$\begin{array}{ll}\text { Nomenclature } & \text { xvii }\end{array}$

1 Introduction $\quad 1$

1.1 Motivation . . . . . . . . . . . . . . . . . 1

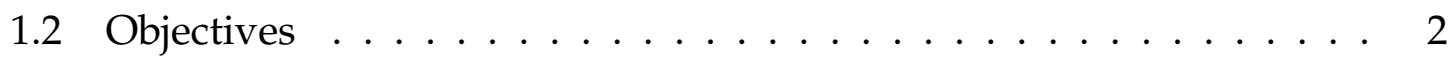

1.3 Focus of the Present Study . . . . . . . . . . . . . 3

2 X-ray Fundamentals $\quad 4$

2.1 X-ray Production ..................... 5

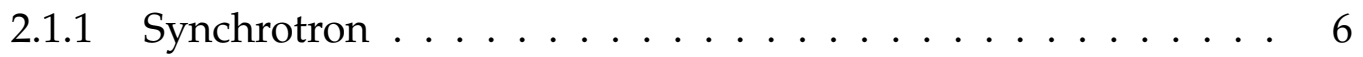

2.1 .2 Tube Generator . . . . . . . . . . . . . . 7

2.1.3 X-ray Photon Production . . . . . . . . . . . . . 10

2.1.4 X-ray Magnification . . . . . . . . . . . . . 12 
2.2 X-ray Photon-Material Interaction $\ldots \ldots \ldots \ldots$

2.2.1 Attenuation Coefficients . . . . . . . . . . . . . . . 23

2.2.2 Spectral Material Attenuation Response . . . . . . . . . . . 25

2.3 X-ray Contrast . . . . . . . . . . . . . . . . . 27

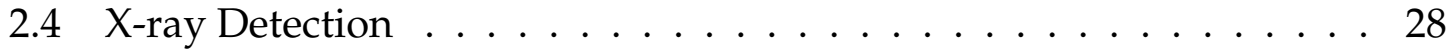

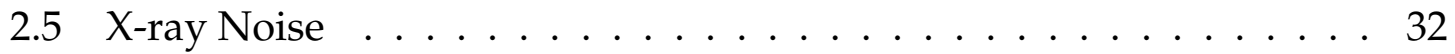

3 Digital Image Correlation $\quad 38$

3.1 Strain Measurement Techniques . . . . . . . . . . . . . . . . . . 38

3.2 Digital Image Correlation $\ldots \ldots \ldots \ldots$

3.2.1 Mathematical Basis . . . . . . . . . . . . . . . . . . . 41

3.2.2 Parameter Space . . . . . . . . . . . . . . . . . 45

3.2 .3 Sources of Error . . . . . . . . . . . . . . . . 47

3.3 X-ray Digital Image Correlation . . . . . . . . . . . . . . . . 52

3.3.1 X-ray Imaging Methods $\ldots \ldots$

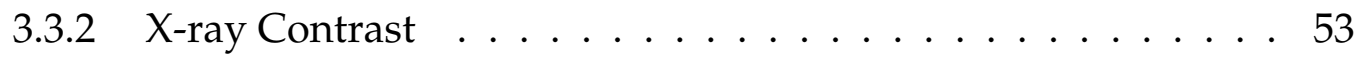

4 Analytical Models $\quad 60$

4.1 Modelling of Marker Contrast . . . . . . . . . . . . . . . 60

4.1.1 Material Attenuation . . . . . . . . . . . . . . . 61

4.1 .2 Layout Dimensions . . . . . . . . . . . . . . . . . 63

$4.1 .3 \quad$ X-ray Spectrum . . . . . . . . . . . . . . . . . 63

4.1.4 Camera Parameters . . . . . . . . . . . . . . . . . . 65

4.1 .5 Specimen Layers . . . . . . . . . . . . . . . . . . 69

4.1.6 Scintillator Properties . . . . . . . . . . . . . 70

4.1.7 Limitations/Assumptions . . . . . . . . . . . . . . . 70

4.2 Out-of-Plane Motion $\ldots \ldots \ldots \ldots \ldots$

4.2 .1 Derivation . . . . . . . . . . . . . . . . 74 
5 Experimental Details $\quad 84$

5.1 X-ray Imaging System . . . . . . . . . . . . . . . . . . . . 84

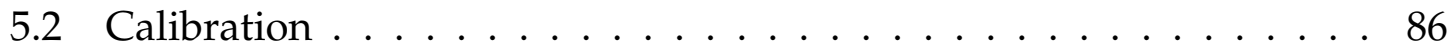

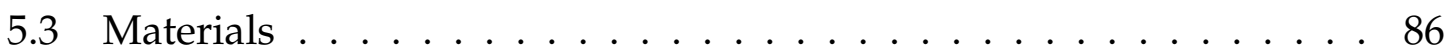

5.3.1 Tissue Surrogate Materials . . . . . . . . . . . . . . . . . . 87

5.3.2 Marker Composition . . . . . . . . . . . . . . 87

5.3.3 Specimen Preparation ................. 88

5.4 Measurement Method . . . . . . . . . . . . . . . . . . 92

5.5 Digital Image Correlation Software . . . . . . . . . . . . . . . 93

6 Results and Discussion $\quad 96$

6.1 Out-of-Plane Motion . . . . . . . . . . . . . . . . . 96

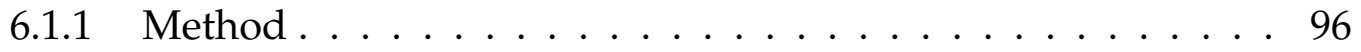

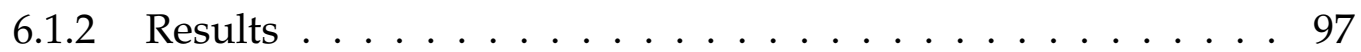

6.1.3 Discussion . . . . . . . . . . . . . . . . 104

6.2 Analytical CNR Tool Validation . . . . . . . . . . . . . . . . 107

6.2.1 CNR DIC Error . . . . . . . . . . . . . . . . 109

6.2.2 Experimental DIC Error . . . . . . . . . . . . . . 117

6.2.3 Analytical Model Validation . . . . . . . . . . . . . . 122

6.3 Preliminary Headform Study . . . . . . . . . . . . . . . . 127

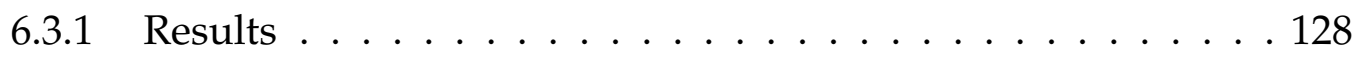

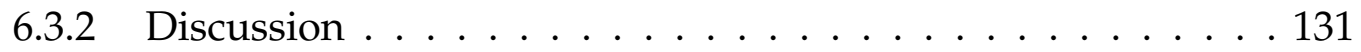

$\begin{array}{lll}7 & \text { Conclusion } & 132\end{array}$

$\begin{array}{ll}\text { List of References } & 137\end{array}$

Appendix A X-ray-Matter Interactions $\quad 144$

A.1 Rayleigh (Coherent) Scatter . . . . . . . . . . . . . . . . . 144 
A.2 Compton (Incoherent) Scatter . . . . . . . . . . . . . . . 145

A.3 Photoelectric Effect . . . . . . . . . . . . . . . . . . . . . 146

$\begin{array}{lll}\text { Appendix B Out-of-Plane Motion: Error Derivation } & 148\end{array}$

$\begin{array}{lll}\text { Appendix C Error Contribution Summary } & 169\end{array}$

C.1 Contrast-to-Noise Ratio . . . . . . . . . . . . . . . . . . . . . . . 169

C.2 Out-of-Plane Motion . . . . . . . . . . . . . . . . . . . 169

C.2.1 Out-of-Plane Translation . . . . . . . . . . . . . . . . . . 170

C.2.2 Out-of-Plane Rotation (1 axis) . . . . . . . . . . . . . . . 171

Appendix D Headform Displacement Plots 176 


\section{List of Tables}

1 Layout dimension definitions $\ldots \ldots \ldots \ldots$

2 Camera software parameters $\ldots \ldots \ldots \ldots \ldots$

3 Camera hardware parameters . . . . . . . . . . . . 67

4 Attenuation path definitions . . . . . . . . . . . . . . . . 69

5 Densities of PDMS-BaSO ${ }^{4}$ Mixtures. . . . . . . . . . . . . . . . . . . . 89

6 Parameters used in DIC out-of-plane translation analysis . . . . . . 98

7 Parameters used in DIC out-of-plane rotation analysis . . . . . . . . 104

8 Single-pixel CNR for artificial images of set marker and background intensities . . . . . . . . . . . . . . . . . . . 110

9 Parameters used in DIC analysis of artificially produced images . . . 112

10 Measured pixel intensity and noise and corresponding CNR estimates118

11 Measured CNR and RMS residual error at several input power levels.119

12 Measured pixel intensity and noise and analytical approximations at several power levels. . . . . . . . . . . . . . . . . . . . . 124

13 Parameters used in DIC analysis of BI ${ }^{2}$ PED headform. . . . . . . . . 129 


\section{List of Figures}

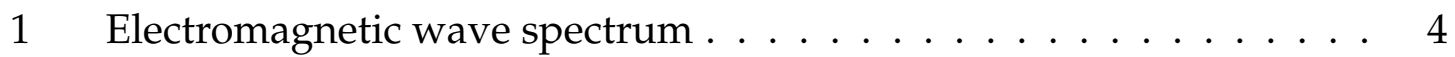

2 Optical and radiographic image of a human hand . . . . . . 5

3 Canada Light Source synchrotron facility $\ldots \ldots \ldots \ldots$

$4 \quad$ X-ray tube generator $\ldots \ldots \ldots \ldots \ldots \ldots$

5 Flash X-ray images of an aluminum sphere impacting aluminum and ceramic fabric . . . . . . . . . . . . . . . . . . 9

6 Rotating anode tube generator schematic . . . . . . . . . . 10

7 Spectrum generated from a tungsten anode generator at $120 \mathrm{kV}_{p} \quad \ldots 11$

8 Process generating bremsstrahlung spectrum . . . . . . . . . . 12

9 Object magnified projection from anode to image on sensor. . . . . . 13

10 Magnification of an element after in-plane translation . . . . . . . 15

11 Infinitesimal first-order strain element and projection definition. . . 16

12 Magnification of an element after in-plane strain . . . . . . . . . 17

13 Magnification element from four points on the focal spot. . . . . . . 19

14 Visualization of edge blur variation through the field of view . . . . 20

15 Anti scatter grid . . . . . . . . . . . . . . . . . 22

16 Illustration of X-ray photon attenuation. . . . . . . . . . . . . . 24

17 Illustration of X-ray photon attenuation through multiple layers of material. . . . . . . . . . . . . . . . . . . . . . . . 24 
18 Incident spectrum and transmitted spectrum through $2.5 \mathrm{~mm}$ aluminum at $140 \mathrm{kV}_{p}$ and 1 mGy KERMA . . . . . . . . . . . . 26

19 X-ray transmission variation through two different cross-sectional

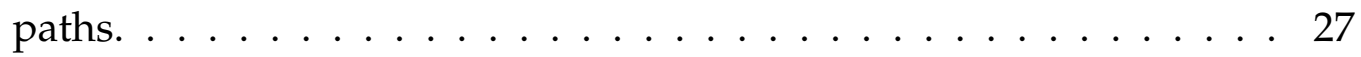

20 Effect of scintillator thickness and reflective back layer on perceived signal at scintillator surface $\ldots \ldots \ldots$

$21 \quad$ X-ray resolution test pattern . . . . . . . . . . . . . . . 31

22 Strain gauge transducer $\ldots \ldots \ldots \ldots \ldots \ldots$

23 Geometric Moiré fringes created by a shift between a reference and a mobile specimen template . . . . . . . . . . . . . . . . . . 39

24 High-contrast painted surface speckle pattern required for DIC . . 40

25 Effect of out-of-plane translation on perceived feature location . . . 49

26 Effect of telecentric lens on perceived image . . . . . . . . . . . 51

27 Effect of out-of-plane rotation on perceived feature location . . . . . 51

28 X-ray phase contrast image of aluminum with surface etching for crack propagation and DIC plots . . . . . . . . . . . . . 54

29 CT reconstruction of aluminum foam . . . . . . . . . . . . 54

30 CT reconstruction of argillaceous rock using naturally occuring contrast and corresponding von-Mises strain . . . . . . . . . . . 55

31 CT reconstruction of wide-grained sand in triaxial compression . . . 56

32 X-ray analysis of ballistic dwell in $\mathrm{SiC}$ penetration . . . . . . . . 57

33 Flash X-ray of steel sphere penetrating polyester with embedded lead shavings $\ldots \ldots \ldots \ldots \ldots \ldots \ldots$

34 X-ray image of embedded tin/polystyrene contrast markers in a

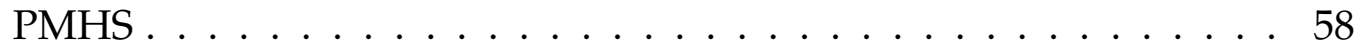

35 Matlab GUI to compute custom material attenuation coefficients. . . 62

36 Matlab GUI to specify experiment dimensions . . . . . . . . . . . 64 
37 Matlab GUI to specify X-ray parameters . . . . . . . . . . . 66

38 Matlab GUI to specify camera parameters . . . . . . . . . . . . . 66

39 Matlab GUI to specify specimen cross section composition . . . . . 70

40 Matlab GUI for scintillator selection and to display representative

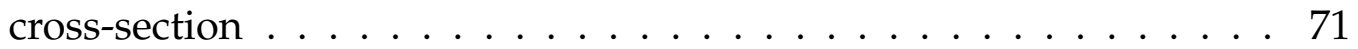

41 Heel effect cause in anode . . . . . . . . . . . . . . . . 72

42 Heel effect intensity distribution $\ldots \ldots \ldots \ldots \ldots 72$

43 First order strain element . . . . . . . . . . . . . . . . . . . 74

44 Projection of the strained element after out-of-plane translation and biaxial rotation. . . . . . . . . . . . . . . . . 75

45 Projection of the strained element after out-of-plane translation. . . . 81

46 Overhead photograph of the high-speed X-ray system at Carleton University. . . . . . . . . . . . . . . . . 86

47 Linear and mass attenuation profile of Barium Sulphate with PDMS at varying concentrations $\ldots \ldots \ldots \ldots \ldots$

48 Photograph of radiographic contrast markers (50 wt $\% \mathrm{BaSO}_{4}, 50$ wt $\%$ Sylgard 184) before embedding in a specimen . . . . . . . . . 90

49 Pre-cast contrast sheet (50 wt $\% \mathrm{BaSO}_{4}, 50 \mathrm{wt} \%$ Humimic gel). . . . . 90

50 Photograph of the rectangular specimen with embedded contrast slice. 91

51 Photograph of the Brain Injury Protection Evaluation Device (BI $\left.{ }^{2} \mathrm{PED}\right)$ headform . . . . . . . . . . . . . . . . . . 92

52 Photograph of the NOCSAE drop used for preliminary impact ex-

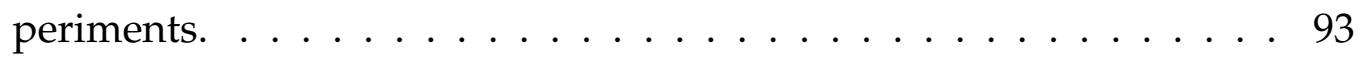

53 Photographs of the Cadex Linear Impactor and mounting fixture . . 94

54 Perceived displacement plots from out-of-plane translation . . . . . 95

55 Quiver plots created from Ncorr displacements plots . . . . . . . . 95

56 DIC strain fields from pure out-of-plane translation by $-3.25 \% \ldots 98$ 
57 Average measured and predicted values of perceived strains caused by out-of-plane translation . . . . . . . . . . . . . . . . . . 99

58 Residual standard error of perceived strains for various relative outof-plane translation . . . . . . . . . . . . . . 100

59 DIC strain fields for out-of-plane rotation by $2.5^{\circ} \ldots \ldots \ldots \ldots$

60 Average measured and predicted values of perceived strains caused by out-of-plane rotation . . . . . . . . . . . . . . . 102

61 Residual standard error perceived strains for various relative outof-plane rotations . . . . . . . . . . . . . . . . 103

62 Flowchart demonstrating the purpose of the analytical model. . . . 108

63 Flowchart demonstrating the application of the analytical model. . . 109

64 Examples of images used in DIC CNR error quantification analysis . 111

65 Sample normal strain fields computed for artificial images . . . . . 113

66 Relative standard error of residual pixel value versus total contrastto-noise ratio with varying strains $\ldots \ldots \ldots \ldots$

67 Flowchart demonstrating the steps required for analytical model calibration. . . . . . . . . . . . . . . . . . . . 117

68 Reference images for experimental error analysis . . . . . . . . . . 120

69 Measured residual RMSe of DIC analyses versus pixel-level contrast-to-noise ratio . . . . . . . . . . . . . . . . 121

70 Root mean squared error of residual (predicted-measured) as a function of pixel-level CNR for artificially and experimentally produced

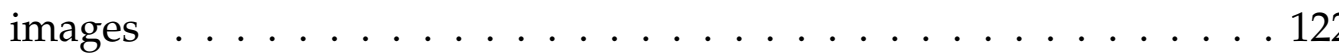

71 Root mean squared error of residual (predicted-measured) as a function of image-level CNR for strain applied to artificially generated images and experimentally produced images . . . . . . . . . . 123 
72 Pixel intensity as a function of electron count estimated by the analytical model camera gain calibration. . . . . . . . . . . . 125

73 Measured versus actual $C N R_{p x}$ for a range of energy levels. . . . . 126

74 Modulation Transfer Function at 2000 frames per second . . . . . . 127

75 Top view of $\mathrm{BI}^{2} \mathrm{PED}$ brain sectioned for marker integration . . . . . 128

76 X-ray image of the BIPED headforms with individually placed markers integrated in a pre-cast brain . . . . . . . . . . . . 129

77 Displacement quiver plot from DIC data for the $\mathrm{BI}^{2} \mathrm{PED}$ headform after a $55 \mathrm{~cm}$ drop captured at $1000 \mathrm{fps} . \quad \ldots \ldots$. . . . . . . . 130

A.1 Illustration of Compton scatter probabilistic emission direction . . 146

B.1 Projection of the strained element after out-of-plane translation and biaxial rotation. . . . . . . . . . . . . . . . . . . . . . . . . . 149

B.2 Infinitesimal strain element and projection definitions . . . . . . 150

C.1 Root mean squared error of residual as a function of image-level CNR for strain applied to artificially generated images and experimentally produced images . . . . . . . . . . . . . . . . 170

C.2 Perceived normal strain versus relative out-of-plane translation $\ldots 170$

C.3 Perceived normal strain parallel to the axis of rotation . . . . . . . 171

C.4 Perceived normal strain perpendicular to the axis of rotation for uniaxial rotation of $2.5^{\circ} \ldots \ldots \ldots \ldots \ldots \ldots \ldots$

C.5 Perceived normal strain perpendicular to the axis of rotation for uniaxial rotation of $5.0^{\circ} \ldots \ldots \ldots \ldots \ldots \ldots$

C.6 Perceived normal strain perpendicular to the axis of rotation for uni-

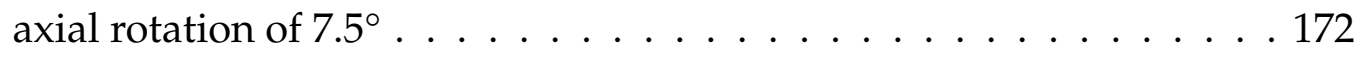

C.7 Perceived normal strain perpendicular to the axis of rotation for uniaxial rotation of $10.0^{\circ} \ldots \ldots \ldots \ldots \ldots \ldots$ 
C.8 Perceived normal strain perpendicular to the axis of rotation for uniaxial rotation of $12.5^{\circ} \ldots \ldots \ldots \ldots \ldots$

C.9 Perceived normal strain perpendicular to the axis of rotation for uniaxial rotation of $15.0^{\circ} \ldots \ldots \ldots \ldots$

C.10 Perceived normal strain perpendicular to the axis of rotation for uniaxial rotation of $17.5^{\circ} \ldots \ldots \ldots$. . . . . . . . . . . . . . . .

C.11 Perceived normal strain perpendicular to the axis of rotation for uniaxial rotation of $20.0^{\circ} \ldots \ldots \ldots \ldots \ldots$

C.12 Perceived normal strain perpendicular to the axis of rotation for uniaxial rotation of $22.5^{\circ} \ldots \ldots \ldots \ldots \ldots$

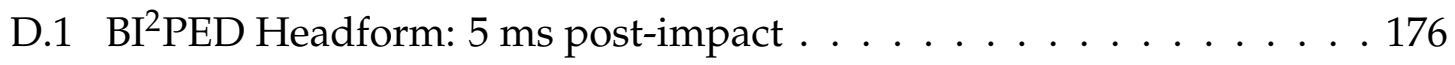

D.2 BI ${ }^{2}$ PED Headform: 10 ms post-impact . . . . . . . . . . . . 177

D.3 BI ${ }^{2}$ PED Headform: 15 ms post-impact . . . . . . . . . . . 177

D.4 BI ${ }^{2}$ PED Headform: 20 ms post-impact $\ldots \ldots \ldots \ldots \ldots \ldots$

D.5 BI ${ }^{2}$ PED Headform: 25 ms post-impact $\ldots \ldots \ldots \ldots \ldots \ldots \ldots$

D.6 BI ${ }^{2}$ PED Headform: 30 ms post-impact . . . . . . . . . . . . . . 179

D.7 BI ${ }^{2}$ PED Headform: 35 ms post-impact . . . . . . . . . . . . . . 179

D.8 BI ${ }^{2}$ PED Headform: 40 ms post-impact . . . . . . . . . . . . 180

D.9 BI ${ }^{2}$ PED Headform: 45 ms post-impact . . . . . . . . . . . . 180

D.10 BI ${ }^{2}$ PED Headform: 50 ms post-impact . . . . . . . . . . . . . 181 


\section{Nomenclature}

\section{Latin Characters}

\begin{tabular}{lll} 
Symbol & Description & Units \\
\hline$A_{p s}$ & Area of speckle on scintillator & {$\left[\mathrm{mm}^{2}\right]$} \\
$a$ & Acceleration & {$\left[\mathrm{m} / \mathrm{s}^{2}\right]$} \\
$a_{i j}$ & Element $i, j$ in rotation matrix $\left[R_{z y x}\right]$ & {$[-]$} \\
$C$ & Contrast & {$[-]$} \\
$C_{C}$ & Cross-Correlation criterion & {$[-]$} \\
$C_{S S D}$ & Sum of Squared Differences Correlation criterion & {$[-]$} \\
$C_{Z N C}$ & Zero-Normalized Cross-Correlation criterion & {$[-]$} \\
$C_{Z N S S D}$ & Zero-Normalized Sum of Squared Differences & \\
& Correlation criterion & {$[-]$} \\
& & {$[-]$} \\
$C N R$ & Contrast-to-Noise Ratio & {$[\mathrm{m} / \mathrm{s}]$} \\
$C$ & Speed of light & {$[\mathrm{mm}]$} \\
$D_{W}$ & Working distance from lens to object.
\end{tabular}




\begin{tabular}{|c|c|c|}
\hline$F$ & Force & {$[\mathrm{N}]$} \\
\hline$F_{f}$ & Camera sensor fill factor & {$[\%]$} \\
\hline$f_{z}$ & Relative out-of-plane translation magnitude & {$[-]$} \\
\hline$f(x, y)$ & Reference subset intensity & {$[-]$} \\
\hline G & Total gain from absorbed energy to pixel value & {$[1 / \mathrm{keV}]$} \\
\hline$g$ & Gain of a specific process & {$[-]$} \\
\hline$g(x, y)$ & Deformed subset intensity & {$[-]$} \\
\hline$h_{s}$ & Camera sensor dimension & {$[\mathrm{mm}]$} \\
\hline$I$ & X-ray flux intensity & [photons] \\
\hline$I_{d}$ & X-ray flux intensity at distance $d$ & [photons] \\
\hline KERMA & Kinetic Energy Released per Mass Air & [Gy] \\
\hline$k$ & Constant: $1.6021 \times 10^{-} 13$ & {$[\mathrm{~Gy} / \mathrm{keV}]$} \\
\hline$L$ & Lens focal length & {$[\mathrm{mm}]$} \\
\hline$m$ & Mass & {$[\mathrm{g}]$} \\
\hline$N(E)$ & Number of photons of energy $E$ & {$[-]$} \\
\hline NPS & Noise Power Spectrum & {$[-]$} \\
\hline$n_{p x}$ & Area spanned by speckle & [pixels] \\
\hline$O_{r}$ & Location of centre of rotation $\left(O_{r, x}, O_{r, y}, O_{r, z}\right)$ & {$[-]$} \\
\hline$P$ & Location of strain element $\left(P_{x}, P_{y}, P_{z}\right)$ & {$[-]$} \\
\hline
\end{tabular}




\begin{tabular}{|c|c|c|}
\hline$P^{\prime}$ & Location of strain element after & \\
\hline & deformation: $\left(P_{x}^{\prime}, P_{y}^{\prime}, P_{z}^{\prime}\right)$ & {$[-]$} \\
\hline$P^{\prime \prime}$ & Location of strain element after deformation and & \\
\hline & out-of-plane motion $\left(P_{x}^{\prime \prime}, P_{y}^{\prime \prime}, P_{z}^{\prime \prime}\right)$ & {$[-]$} \\
\hline$P X$ & Pixel intensity & {$[-]$} \\
\hline$Q$ & Projected location of strain element $\left(Q_{x}, Q_{y}\right)$ & {$[-]$} \\
\hline$Q^{\prime}$ & Projected location of strain element after & \\
\hline & deformation: $\left(Q_{x}^{\prime}, Q_{y^{\prime}}^{\prime}\right)$ & {$[-]$} \\
\hline$Q^{\prime \prime}$ & Projected location of strain element after & \\
\hline & deformation and out-of-plane motion $\left(Q_{x}^{\prime \prime}, Q_{y}^{\prime \prime}\right)$ & {$[-]$} \\
\hline$Q E$ & Camera quantum efficiency & {$[\%]$} \\
\hline$R$ & Image location of strain element $\left(R_{x}, R_{y}\right)$ & {$[-]$} \\
\hline$R^{\prime}$ & Image location of strain element after & \\
\hline & deformation: $\left(R_{x}^{\prime}, R_{y}^{\prime}\right)$ & {$[-]$} \\
\hline$R^{\prime \prime}$ & Image location of strain element after deformation & \\
\hline & and out-of-plane motion $\left(R_{x}^{\prime \prime}, R_{y}^{\prime \prime}\right)$ & {$[-]$} \\
\hline$\left[R_{z} y x\right]$ & $3 \mathrm{D}$ rotation matrix about $z^{\prime}, y^{\prime}$, then $x^{\prime}$ axes & {$[-]$} \\
\hline$r$ & Record frame rate & [frames/s] \\
\hline$S$ & Signal & {$[-]$} \\
\hline
\end{tabular}




$\begin{array}{lll}T & \text { Thickness } & {[\mathrm{cm}]} \\ u(x, y)_{n} & \text { n-order x-deformation shape function } & {[\mathrm{mm}]} \\ V_{i} & \text { volume fraction } & {[-]} \\ v(x, y)_{n} & \text { n-order y-deformation shape function } & {[\mathrm{mm}]} \\ W_{i} & \text { mass fraction } & {[-]} \\ X & \text { Location of point } Q \text { on scintillator in } x \text { direction } & {[\mathrm{mm}]} \\ x & \text { Location of point } P \text { in 3D space along } x \text { axis } & {[\mathrm{mm}]} \\ Y & \text { Location of point } Q \text { on scintillator in } y \text { direction } & {[\mathrm{mm}]} \\ y & \text { Location of point } P \text { in 3D space along } y \text { axis } & {[\mathrm{mm}]} \\ Z & \text { Distance from source to scintillator } & {[\mathrm{mm}]} \\ Z_{a} & \text { atomic number } & {[-]} \\ Z & \text { Location of point } P \text { in 3D space along } z \text { axis } & {[\mathrm{mm}]}\end{array}$

\section{Greek Characters}

\begin{tabular}{lll} 
Symbol & Description & Units \\
\hline$\Delta$ & Variation & {$[-]$} \\
$\varepsilon$ & Appliedstrain & {$[-]$} \\
$\varepsilon^{\prime}$ & Perceived strain & {$[-]$}
\end{tabular}




$\begin{array}{lll}\zeta & \text { Deformation shape function in the y direction } & {[\text { length }]} \\ \eta_{x p} & \text { X-ray production efficiency } & {[-]} \\ \theta_{i} & \text { Rotation angle about } i \text { axis } & {\left[{ }^{\circ}\right]} \\ \mu & \text { Linear attenuation coefficient } & {[1 / \mathrm{cm}]} \\ \mu / \rho & \text { Mass attenuation coefficient } & {\left[\mathrm{cm}^{2} / \mathrm{g}\right]} \\ \mu / \rho_{e n} & \text { Mass energy absorption coefficient } & {\left[\mathrm{cm}^{2} / \mathrm{g}\right]} \\ v & \text { Poissons ratio } & {[-]} \\ \xi & \text { Deformation shape function in the x direction } & {[\mathrm{length}]} \\ \rho & \text { Density } & {\left[\mathrm{g} / \mathrm{cm}^{3}\right]} \\ \sigma^{2} & \text { Variance } & {[-]} \\ \Phi(E) & \text { Spectral photon fluence } & {\left[\mathrm{photons} / \mathrm{mm}^{2}\right]} \\ \Psi(E) & \text { Spectral energy fluence } & {\left[\mathrm{keV} / \mathrm{mm}^{2}\right]} \\ \Omega & \text { Solid angle } & {[\mathrm{steradians}]}\end{array}$

\section{Subscripts}

Symbol Description

$0 \quad$ Original, incident

$a b \quad$ Absorbed by the scintillator

at Attenuated by a material 


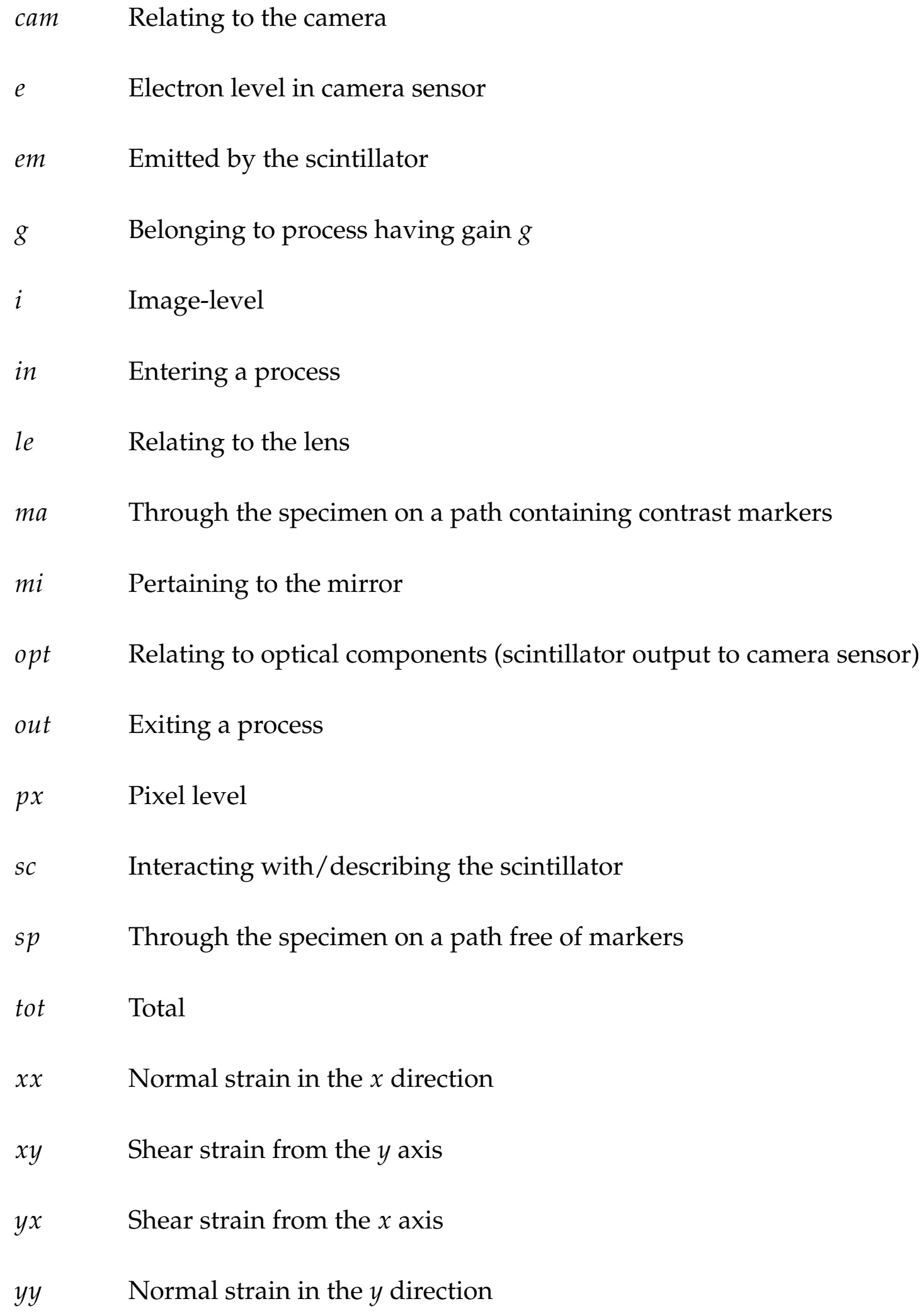


List of Abbreviations

\begin{tabular}{|c|c|}
\hline Abbreviation & Description \\
\hline $\mathrm{BaSO}_{4}$ & Barium Sulphate \\
\hline $\mathrm{BI}^{2} \mathrm{PED}$ & Brain Injury Protection Evaluation Device \\
\hline $\mathrm{CC}$ & Cross Correlation \\
\hline CCD & Charge-Couple Device \\
\hline CLS & Canada Light Source \\
\hline CMOS & Complementary Metal-Oxide-Semiconductors \\
\hline CNR & Contrast-to-Noise Ratio \\
\hline CsI(Tl) & Thallium-doped Cesium Iodide \\
\hline $\mathrm{CT}$ & Computed Tomography \\
\hline CTF & Contrast Transfer Function \\
\hline DIC & Digital Image Correlation \\
\hline DRDC & Defence Research and Development Canada \\
\hline DVC & Digital Volume Correlation \\
\hline FOV & Field of View \\
\hline FPS & Frames per Second \\
\hline GUI & Graphical User Interface \\
\hline IEC & International Electrotechnical Commission \\
\hline
\end{tabular}




$\begin{array}{ll}\text { KERMA } & \text { Kinetic Energy Released per Mass Air } \\ \text { MTF } & \text { Modulation Transfer Function } \\ \text { NIST } & \text { National Institue of Standards and Technology } \\ & \text { National Operating Committee on } \\ \text { NPS } & \text { Standards for Athletic Equipment } \\ \text { PCI } & \text { Noise Power Spectrum } \\ \text { PDMS } & \text { Polydimethylsiloxane } \\ \text { PIV } & \text { Particle Image Velocimetry } \\ \text { PMHS } & \text { Post-Mortem Human Surrogate } \\ \text { PMMA } & \text { Poly(methyl methacrylate) } \\ \text { SSD } & \text { Sum of Squared Differences } \\ \text { TASMIP } & \text { Tungsten Anode Spectral Model using } \\ \text { XDIC } & \text { Interpolating Polynomials } \\ \text { UV } & \text { UltraViolet } \\ & \text { Photon Cross Sections Database } \\ \text { X-ray Digital Image Correlation }\end{array}$




\section{Chapter 1}

\section{Introduction}

\subsection{Motivation}

Characterizing and understanding material behaviour are the foundations of engineering science. Notably, correct prediction of the deformation and strain of a material under static or dynamic loading conditions has allowed for the construction of complex structures and systems. Much of the basis of material science was built by examining the results of physical experiments. In many studies, particularly for dynamic applications, these experiments involve irreversible plastic deformation or destructive testing, making repeated tests costly or infeasible.

Computational models have been developed as an alternative to physical experimentation as a wide range of conditions can be simulated, including dynamic impact events; however, computational models require validation using real-world experimental data to provide a basis for extrapolation to novel loading conditions. The high-speed X-ray facility at Carleton University was designed to generate unique head-injury data in cadaveric studies. Impact-induced brain deformation will be measured to increase understanding of tissue material properties in dynamic conditions, with the intention of improving understanding of 
the mechanical causes of concussion and head-injury. The material models developed during these experiments will be used by researchers developing advanced biofidelic head injury models for validation and verification.

This thesis describes the unique high-speed X-ray facility at Carleton University, including its design, implementation, and limitations. A method of quantifying displacement and strain fields of optically inaccessible internal structures including brain tissue was developed and is presented herein. The implementation and errors of the novel cineradiographic measurement method must be understood in preparation for upcoming cadaveric studies and future helmet evaluation using biofidelic headforms.

\subsection{Objectives}

The work described in this thesis is intended to assist in the design of experiments using the high-speed X-ray facility. The large parameter space of such studies requires in-depth understanding of the constraints, limitations and assumptions used to develop the system. Therefore, the optimal experimental set-up will require compromise between image quality, experimental objectives, physical limitations and reproducible results. The balance of input parameters must be understood in preparation for upcoming cadaveric studies. Specifically, this work aims to accomplish the following objectives:

1. To finalize the design and implementation of the high-speed X-ray lab.

2. To develop a method of designing radiographic contrast markers for displacement and strain measurement of optically inaccessible planes.

3. To validate experimental methodology in preparation for post-mortem head surrogate studies and for helmet evaluation using biofidelic headforms.

4. To identify limitations and quantify expected experimental errors to manage their influence on results to generate the highest quality data possible. 
5. To provide technical recommendations for future experiments conducted using the high-speed X-ray facility.

\subsection{Focus of the Present Study}

The present study is comprised of the following chapters to discuss background theory, relevant literature, development and validation of analytical models, experimental methodology, results and limitations:

Chapter 1: Introduction - Underlying motivation for facility development and research objectives.

Chapter 2: X-ray Fundamentals - Physical explanation of X-ray production, interaction with matter and detection methods.

Chapter 3: Digital Image Correlation - Introduction to DIC, including mathematical basis, parameters, and sources of error. Special focus on X-ray applications.

Chapter 4: Analytical Models - Development of the contrast-to-noise ratio computation tool, and out-of-plane motion error derivation.

Chapter 5: Experimental Details - Technical details regarding the high-speed X-ray facility, specimen preparation and analysis software.

Chapter 6: Results and Discussion - Noise and out-of-plane motion error analyses. Preliminary discussion on implementation with biofidelic headforms.

Chapter 7: Conclusion - Closing remarks and technical recommendations for future implementation with considerations for upcoming cadaveric studies. 


\section{Chapter 2}

\section{X-ray Fundamentals}

X-rays are electromagnetic waves having more energy than ultraviolet (UV) waves and typically less than $\gamma$ waves, as shown on the electromagnetic spectrum in Fig. 1 [1]. Both $X$ - and $\gamma$-rays are types of ionizing radiation, having sufficient energy strip electrons from atoms, though they differ in origin [2]. X-rays are produced during interactions with the atomic shells (i.e., electrons) and $\gamma$-rays are generated from processes involving the nucleus (ie. radioactive decay) [2].

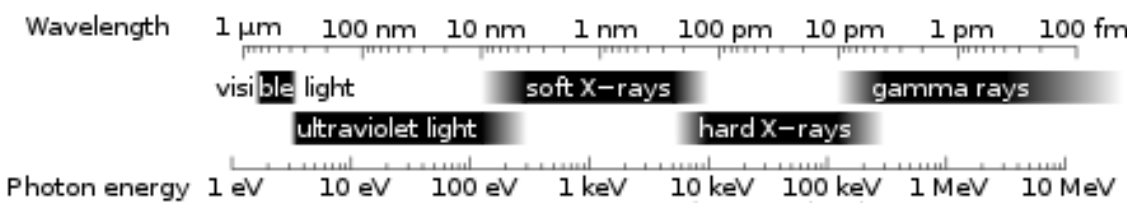

Figure 1: Electromagnetic wave spectrum. Adapted from [1]

X-rays are widely implemented for medical diagnostics; projection radiography, for example, is the measurement of photons transmitted through (or, absorbed) by an object [3]. The imaging specimen is located between the $X$-ray source and sensor. Individual projected images (radiography) or 3D computed tomography $(\mathrm{CT})$ reconstructions using several images captured at different angles are commonly used for medical diagnosis [3]. 

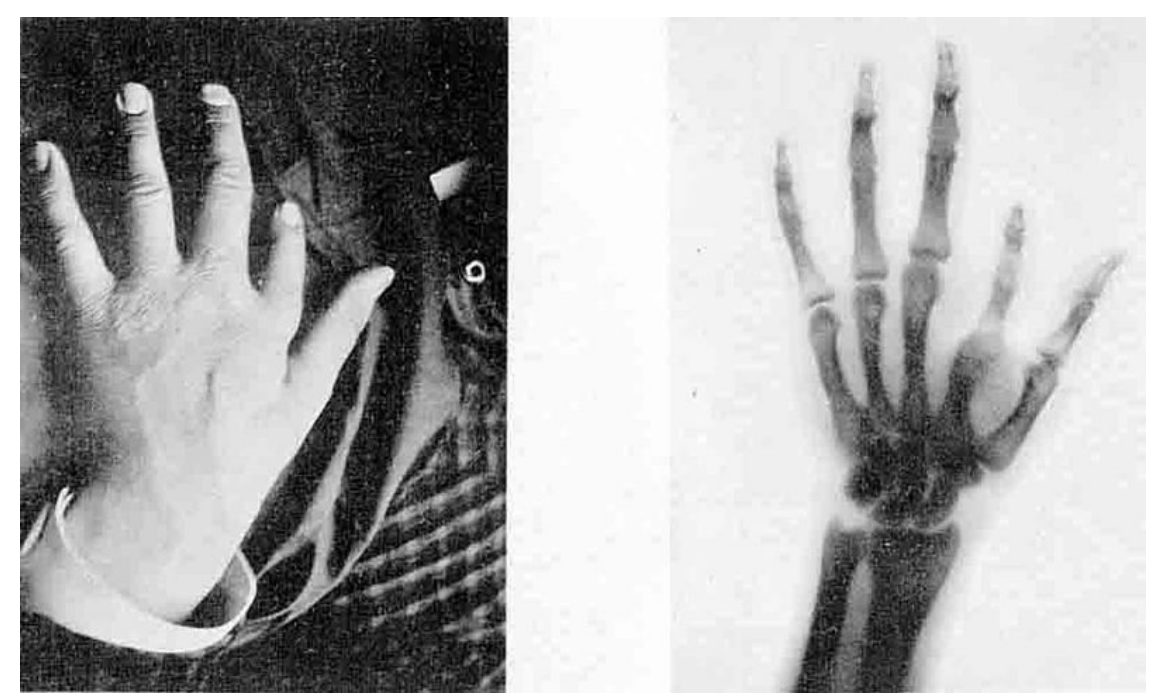

Figure 2: Optical and radiographic image of a human hand. Published in 1896 [4].

The hand imaged optically and radiographically in Fig. 2 illustrates the use of both methods. Electromagnetic wavelength determine both the probability and type of interactions with matter [3]. Higher energy waves (smaller wavelength) are less likely to interact with matter. Therefore X-rays, having relatively high energy levels may be transmitted through a material that would cause visible light to scatter [2], allowing radiography to image optically inaccessible internal structures such as bone in Fig. 2. As a result, methods used to produce and detect electromagnetic waves vary depending on their energy levels.

The radiograph in Fig. 2 demonstrates the clear contrast between bone and soft tissue (i.e., muscle and fat) [4]. This contrast between materials allows medical professionals to identify fractures in bone or to help diagnose other conditions such as cancer or pneumonia.

\subsection{X-ray Production}

X-rays are used in several fields including for medical diagnostic and material composition analyses. Based on experimental and practical requirements, $\mathrm{X}$-rays 
can be produced using one of two methods: Synchrotrons or tube generators.

\subsubsection{Synchrotron}

Synchrotron facilities, such as the Canada Light Source (CLS), in Saskatoon, Canada, are particle accelerators that produce brilliant light (infrared, visible, ultraviolet, or X-ray). Charged particles (i.e., electrons) are accelerated to approach the speed of light, and their trajectories are subsequently deviated using magnetic fields [5], [6]. The acceleration of charged particles as their trajectory is deviated results in the emission of photons at specific wavelengths. Synchrotrons are able to produce high flux beams of specific spectra. The 05ID-2 beamline at the CLS for example, which is closest in emission levels to medical X-rays (25-150 keV), produces sharp spectra with small variation $\left(\Delta \mathrm{E} / \mathrm{E}: 10^{-} 3\right)$ [5], [7].

Synchrotron use has practical limitations for some medical and diagnostic applications. For example, the Canada Light Source (Fig. 3) is a large multi-use facility. Gaining regular access to a synchrotron is difficult and experiments must be planned in advance, thus it is not feasible to conduct a comprehensive study involving fresh cadaveric specimens at a synchroton. Second, the field of view is limited. The 05ID-2 beamline, discussed above, has a field of view of only $220 \mathrm{~mm} \times 11 \mathrm{~mm}$ [5], and require several sequential exposures to image larger specimens. This is impractical in dynamic studies when the specimen is larger than the field of view and there is expected variability between repeated tests. The limitations of synchrotron $\mathrm{X}$-ray generators require an alternate production method for certain applications. 


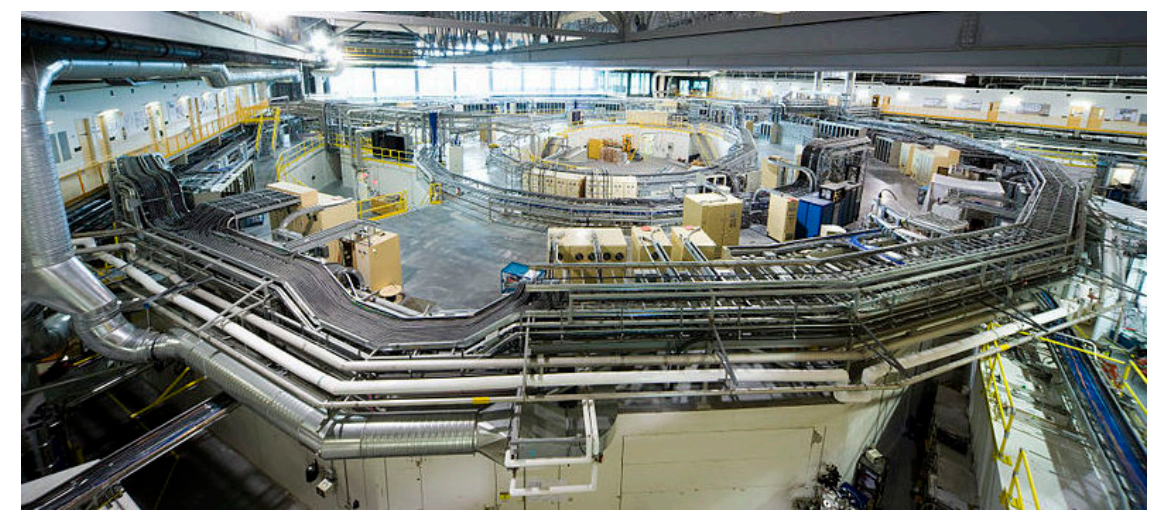

Figure 3: Canada Light Source synchrotron facility [6].

\subsubsection{Tube Generator}

An alternate method of X-ray production to synchrotron facilities is the X-ray tube generator, shown schematically in Figure 4 [7]. Electrons are accelerated in a tube under vaccum from a cathode toward an anode, reaching a precise location called the focal spot. Interactions between the incident electrons and the atoms comprising the focal spot generate X-ray radiation. The electron flux (current) represents the quantity of electrons traveling from cathode to anode, and thus, the quantity of X-ray photons produced. The tube voltage (potential) influences the acceleration rate, and therefore the energy acquired by electrons before reaching the anode. Higher energy incident electrons results in emmitance of higher energy $X$-rays [7].

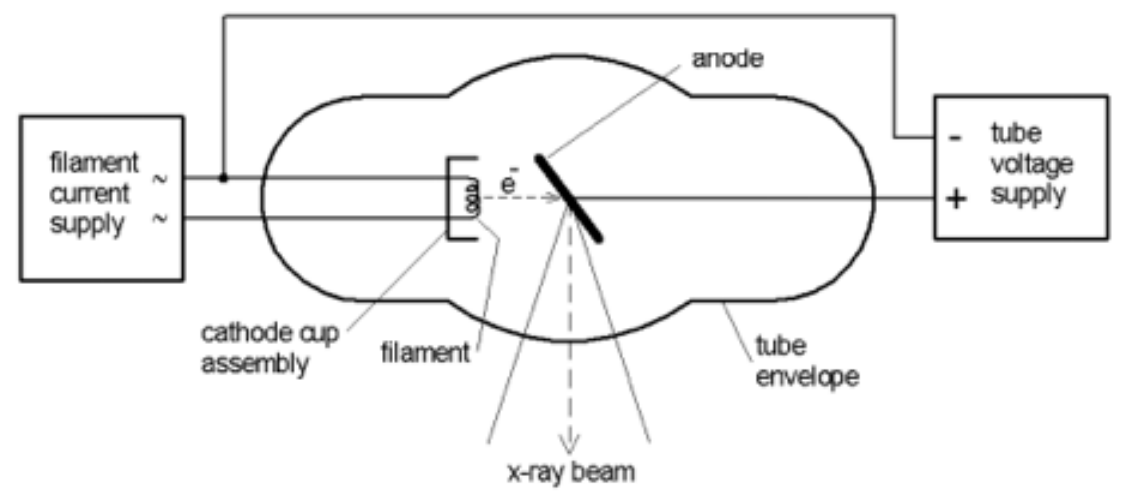

Figure 4: X-ray tube generator, OIAEA [8]. 
Projection radiography is named for the conical expansion of $X$-ray beams generated from electrons hitting the focal spot. The projection angle is dependent on the angle of incidence on the focal spot, which is typically rotated $6-22^{\circ}$ from perpendicular to the incident electron flux in medical applications [7] . Geometry of the focal spot has a significant influence on image shape, quality, and intensity distribution, as discussed later in Sec. 2.1.4 [7].

X-ray production using tube generators is inefficient; only a fraction of the energy of electrons incident on the focal spot contributes to X-ray photon production, with the remainder lost as heat. The $\mathrm{X}$-ray production efficiency, $\eta_{x p}$, is the ratio of electron energy used to generate X-rays to the electron energy lost as heat, and is approximated using the following equation [9]:

$$
\eta_{x p}=k V \cdot Z_{a} \cdot 10^{-6}
$$

The energy transfer efficiency is proportional to the atomic number of the anode, $Z_{a}$ and input voltage, $\mathrm{kV}$, expressed in kilovolts. For medical diagnostics, the input power levels are typically in the range of $25-150 \mathrm{keV}$ [7]. Eq. 1 highlights the extreme inefficiency of tube generators, in fact, during X-ray production at high power levels, the energy lost as heat can melt the focal spot. Therefore, to allow for higher power applications and increased production efficiency, the anode is typically produced of a material such as tungsten with a high atomic number (74) and melting temperatures $\left(3,422^{\circ} \mathrm{C}\right)$ to prevent tube damage and maximize conversion efficiency [9].

A typical tube used for a single rapid exposure is called a flash X-ray generator. For example, Scandiflash systems are used for high power 20-35 ns exposures [10]. Although input voltage of 75-1,200 kV and amperage of 2-10 kA are achievable, 
the high energy limits exposure to very short times to prevent focal spot damage [10]. The nanosecond scale exposure times of flash $X$-ray allow for imaging of high-speed dynamic events including ballistics with no motion blur. For example, three sequential X-ray images from different angles show the result of an aluminum sphere penetrating layers of aluminum and ceramic fabric in Fig. 5 [11]. Multiple orthogonal flash systems are required to produce sequential images with fixed fiducial markers used for image alignment.

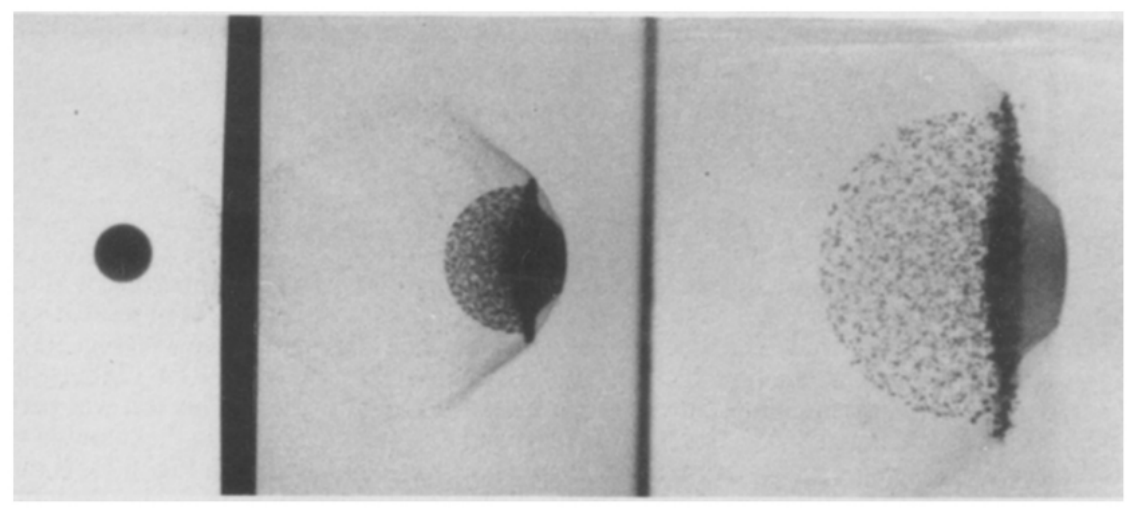

Figure 5: Sequential flash $X$-ray images of an aluminum sphere impacting aluminum and ceramic fabric at $6.6-6.9 \mathrm{~km} / \mathrm{s}$ [11].

Rotating anode generators are used for continuous or fluoroscopic cineradiography. Rotating at high speeds increases the total focal spot area for better heat dissipation while maintaining the same X-ray production window. An schematic of a rotating anode is shown in Fig. 6 [12].

The size of the focal spot is tube specific, and is selected based on the application and influences the imaging quality and peak power level. Increasing the focal spot size allows for greater heat dissipation, allowing for a higher incident energy flux. Energy levels are dependent on the current, influencing the quantity of X-ray photons created, the voltage, changing the energy level of generated photons, and the exposure period. The result of increasing current and voltage is an increase in 


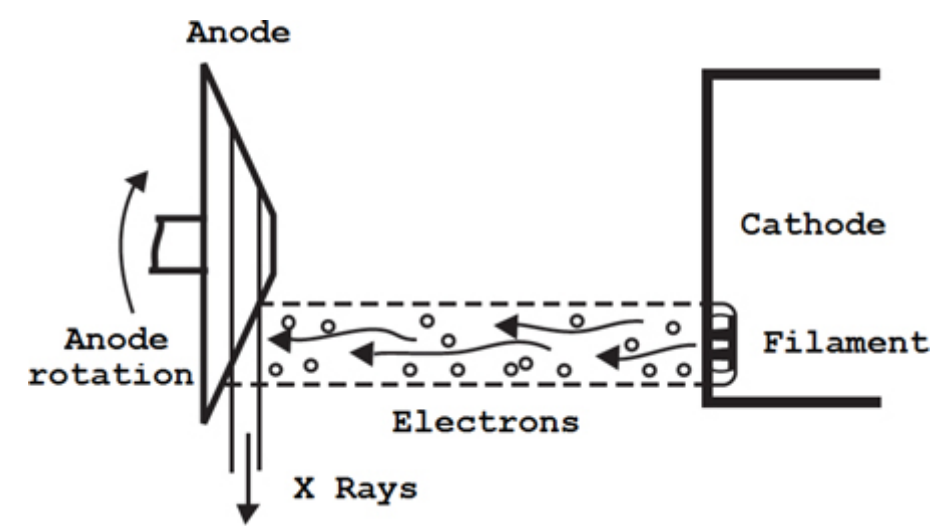

Figure 6: Rotating anode tube generator schematic [12].

brightness and contrast, respectively [2]. Therefore, increasing focal spot size will allow for more brilliant $\mathrm{X}$-ray beams; however, it will also decrease image sharpness.

\subsubsection{X-ray Photon Production}

Focal spot composition plays a critical role in photon generation. Contrarily to synchrotron facilities that can generate precise energy beams, anodes create broad spectra as shown in Fig. 7 using [13] with data from [14].

The spectral output of an X-ray tube, as shown in Figure 7, is the superposition of two principle components: The bremsstrahlung (braking radiation), is the wide peak between approximately $10 \mathrm{keV}$ and the maximum photon energy or peak power (defined in $\mathrm{kV}_{p}$ ), originating from electron-atom interaction; and the characteristic peaks, caused by electron-electron interactions, are unique based on the focal spot composition [13], [2]. The bremsstrahlung is generated by accelerated electrons passing close to the atomic nuclei of anode. The close proximity results in a strong electrostatic attraction between the negative electron and positive nucleus [3]. The electron is decelerated as its trajectory is deviated, resulting in the 


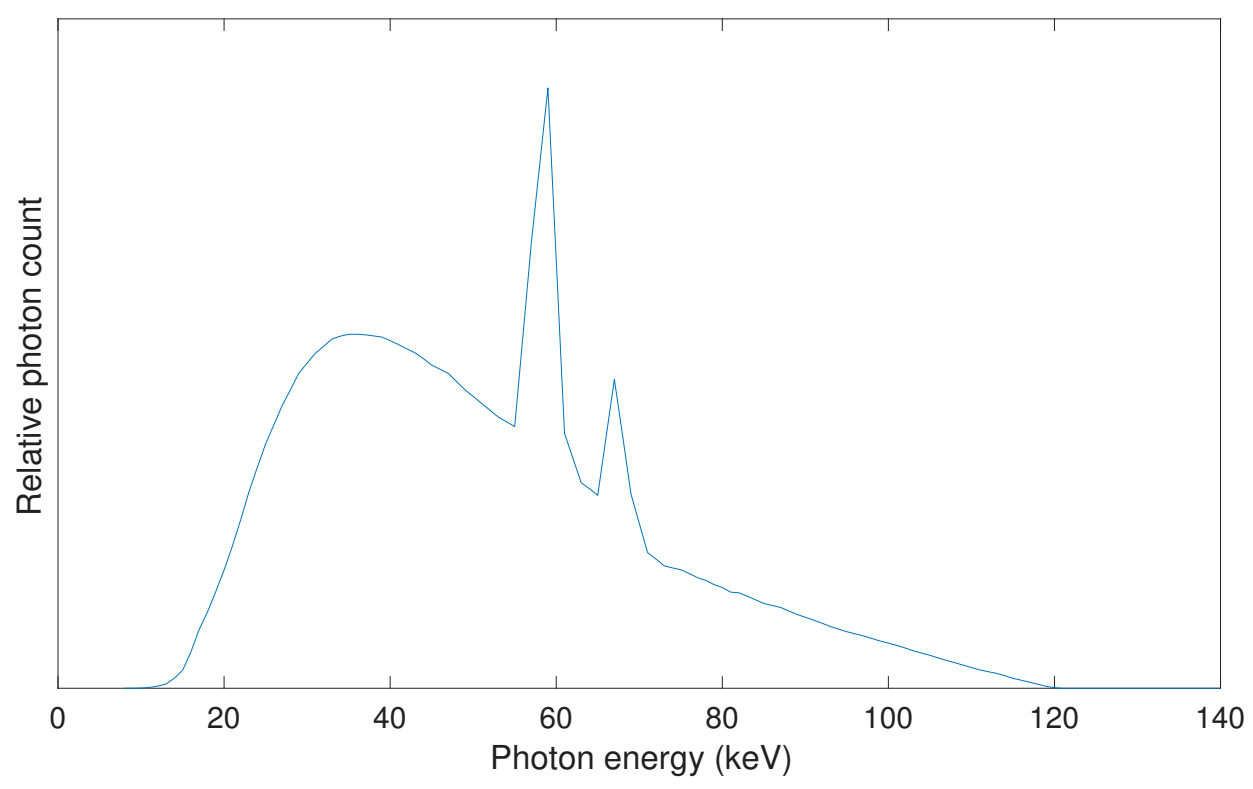

Figure 7: Spectrum generated from a tungsten anode generator at $120 \mathrm{kV}_{p}$ [13].

emission of an X-ray photon due to conservation of energy as shown in Fig. 8 [2].

The characteristic peaks are dependent on the atomic composition of the anode. During X-ray production, an incident electron has sufficient energy to ionize an electron from an inner orbital. When this occurs, an electron from a higher orbital must take its place, resulting in the emission of a photon. Due to conservation of energy, the energy of the emitted photon will be equal to the difference in energy lost by the electron as it moved to a lower orbital [3]. If the orbital transition energy is of sufficiently high, the emitted photon will be an X-ray. As there are a finite number of orbitals, each with known energy levels for a given element, there are also a finite number of possible orbital transition energies for a specific element, making them characteristic energies. A focal spot composed of tungsten produces a spectrum such as that shown in Fig. 7, with a bremsstrahlung peak determined by the peak power level and characteristic energies of approximately 55 and $70 \mathrm{keV}$ [13], [2].

The remainder of the discussion in this thesis will focus on a rotating anode 


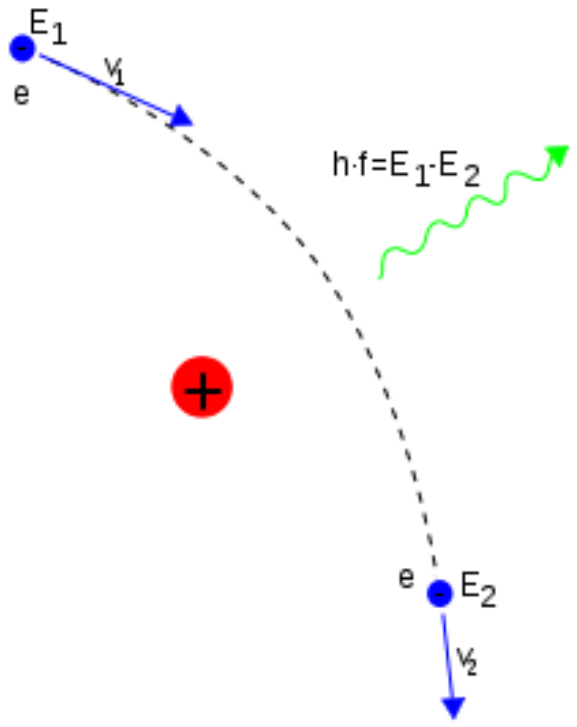

Figure 8: Process generating bremsstrahlung spectrum [2].

X-ray tube generator, reflecting our experimental facility at Carleton University.

\subsubsection{X-ray Magnification}

The location of the object relative to the source and sensor will influence the perceived image. X-ray photons produced at the focal spot are projected towards the object in a cone-like expansion [2]. The projection shape results in linear magnification of the object on the image plane [3]. In order to understand the effects of object placement on image magnification, the focal spot will be approximated as a point source as shown in Fig. 9, and the object is represented as an infinitesimal strain element located at $P$ with dimensions $d x$ by $d y$. The following is an extention of $1 \mathrm{D}$ discussion in [3], [2], and [15], where the object is now shown as 2D.

The coordinates of point, $P$ is

$$
P=\left(P_{x}, P_{y}, P_{z}\right)=(x, y, z)
$$




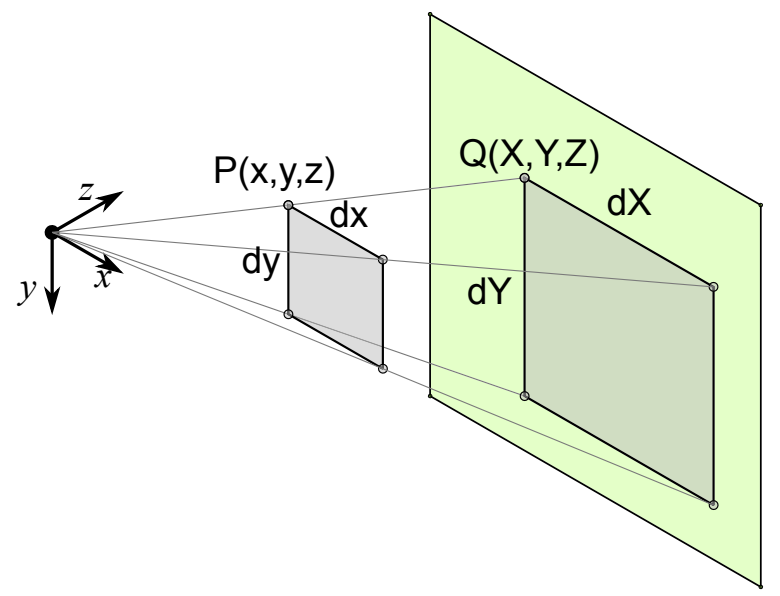

Figure 9: Object magnified projection from anode to image on sensor.

The above point coordinates represent the physical location of the object; however, X-rays produced from a tube project the object, linearly magnifying it onto the sensor plane at $Z$, using the following [3]:

$$
Q=(X, Y, Z)=\left(P_{x} \frac{Z}{P_{z}}, P_{y} \frac{Z}{P_{z}}, Z\right)=\left(x \frac{Z}{z}, y \frac{Z}{z}, Z\right)
$$

The strain element is deformed through directional position dependent functions, $u(x, y)$ and $v(x, y)$. Point $P$ is deformed to $P^{\prime}$ by:

$$
P^{\prime}=P+(u(x, y), v(x, y), 0)=(x+u(x, y), y+v(x, y), z)
$$

From Eq. 3, the object projection on the sensor plane after deformation becomes:

$$
Q^{\prime}=\left(P_{x}^{\prime} \frac{Z}{P_{z}^{\prime}}, P_{y}^{\prime} \frac{Z}{P_{z}^{\prime}}, Z\right)=\left((x+u(x, y)) \frac{Z}{z},(y+v(x, y)) \frac{Z}{z}, Z\right)
$$

Expressed differently, the deformation from original points $P$ and $Q$ to final points $P^{\prime}$ and $Q^{\prime}$ are: 


$$
\begin{aligned}
& u(x, y)=P_{x}^{\prime}-P_{x} \\
& v(x, y)=P_{y}^{\prime}-P_{y}
\end{aligned}
$$

and

$$
\begin{aligned}
& U(x, y)=Q_{x}^{\prime}-Q_{x}=\left(P_{x}^{\prime}-P_{x}\right) \frac{Z}{z} \\
& V(x, y)=Q_{y}^{\prime}-Q_{y}=\left(P_{y}^{\prime}-P_{y}\right) \frac{Z}{z}
\end{aligned}
$$

Where $U(x, y)$ and $V(x, y)$ are the perceived deformations on the sensor plane. The displacement of point $P$ to $P^{\prime}$ is trivially expressed as a zero-order deformation function corresponding to a two-directional in-plane translation of $u$ and and $v$, in the $x$ and $y$ directions respectively, as shown in Fig. 10.

$$
\begin{aligned}
& u_{0}(x, y)=u \\
& v_{0}(x, y)=v
\end{aligned}
$$

For which the resulting translation of the sensor projection becomes:

$$
\begin{aligned}
U_{0}(x, y) & =u \frac{Z}{z} \\
V_{0}(x, y) & =v \frac{Z}{z}
\end{aligned}
$$

Alternatively, the displacement may not be uniform throughout the field (i.e., $u(x, y) \neq u$ and $v(x, y) \neq v)$. For example, a first-order deformation is applied to 


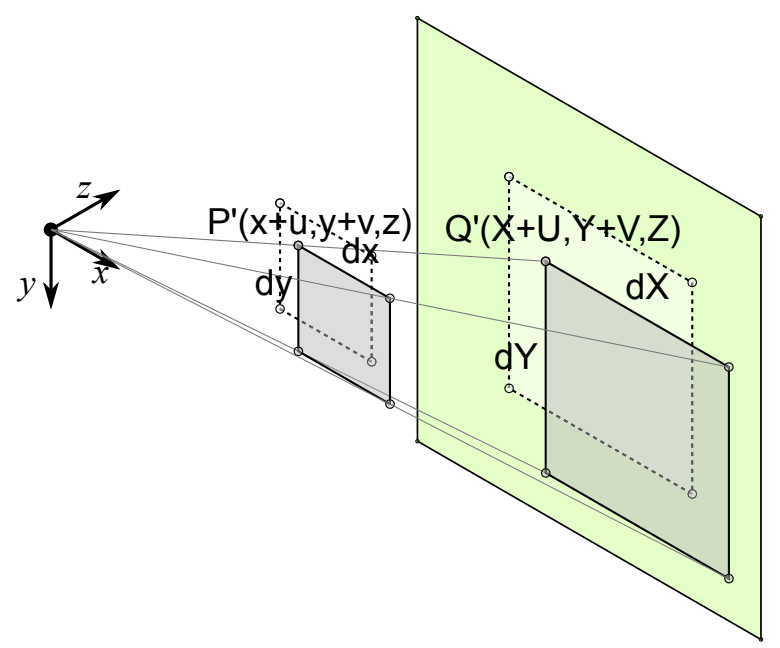

Figure 10: Magnification of an element after in-plane translation

the element shown in Fig. 11 with projection shown in Fig. 12, using the following first-order function:

$$
\begin{aligned}
& u_{1}(x, y)=u+\frac{\partial u}{\partial x} d x+\frac{\partial u}{\partial y} d y \\
& v_{1}(x, y)=v+\frac{\partial v}{\partial x} d x+\frac{\partial v}{\partial y} d y
\end{aligned}
$$

From Fig. 11 and Eq. 16 and 17, the associated first-order strains (i.e., displacement gradients) become the following::

$$
\begin{array}{r}
\varepsilon_{x x}=\frac{\partial u}{\partial x} \\
\varepsilon_{y y}=\frac{\partial v}{\partial y} \\
\varepsilon_{x y} \approx \tan \varepsilon_{x y}=\frac{\frac{\partial u}{\partial y}}{1+\frac{\partial v}{\partial y}} \\
\varepsilon_{y x} \approx \tan \varepsilon_{y x}=\frac{\frac{\partial v}{\partial x}}{1+\frac{\partial u}{\partial x}}
\end{array}
$$




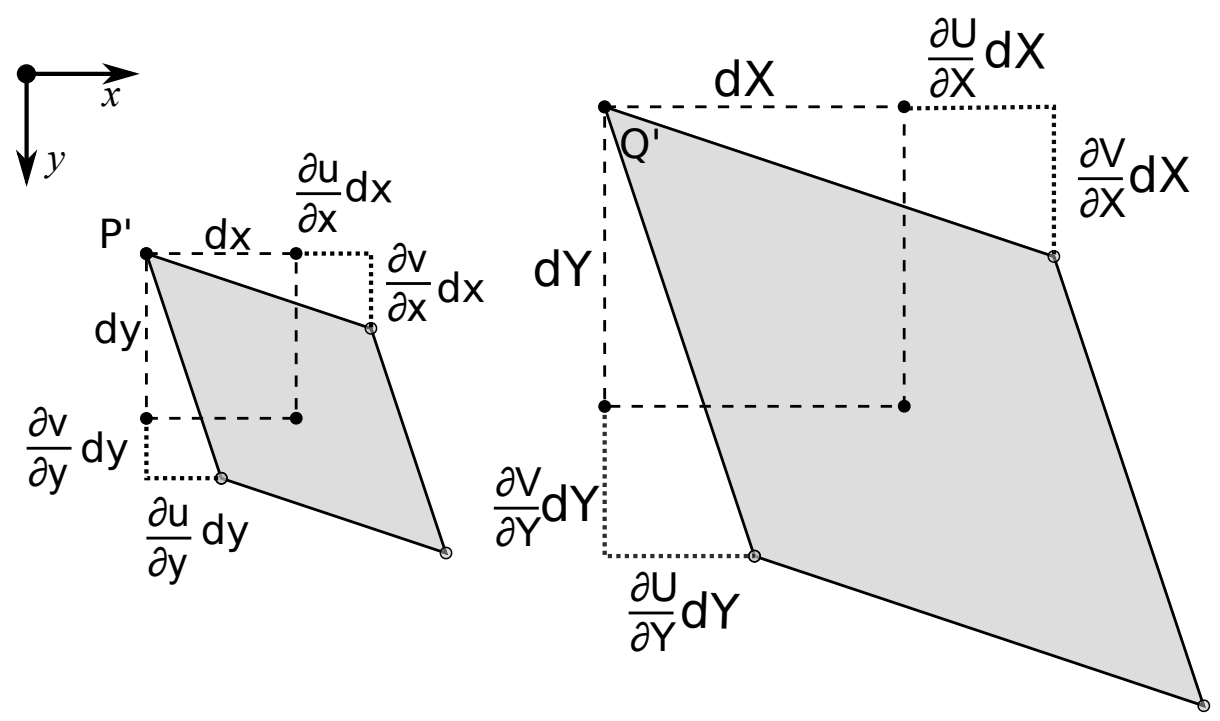

Figure 11: Infinitesimal first-order strain element and projection definition.

Extending Eq. 9 and 10, the following displacements and strains are determined:

$$
\begin{aligned}
& U(x, y)=u(x, y) \frac{Z}{z} \\
& V(x, y)=v(x, y) \frac{Z}{z}
\end{aligned}
$$

The partial derivatives are expressed in terms of $X$ and $Y$ to determine the firstorder strains relative to the original projection onto the sensor plane: 


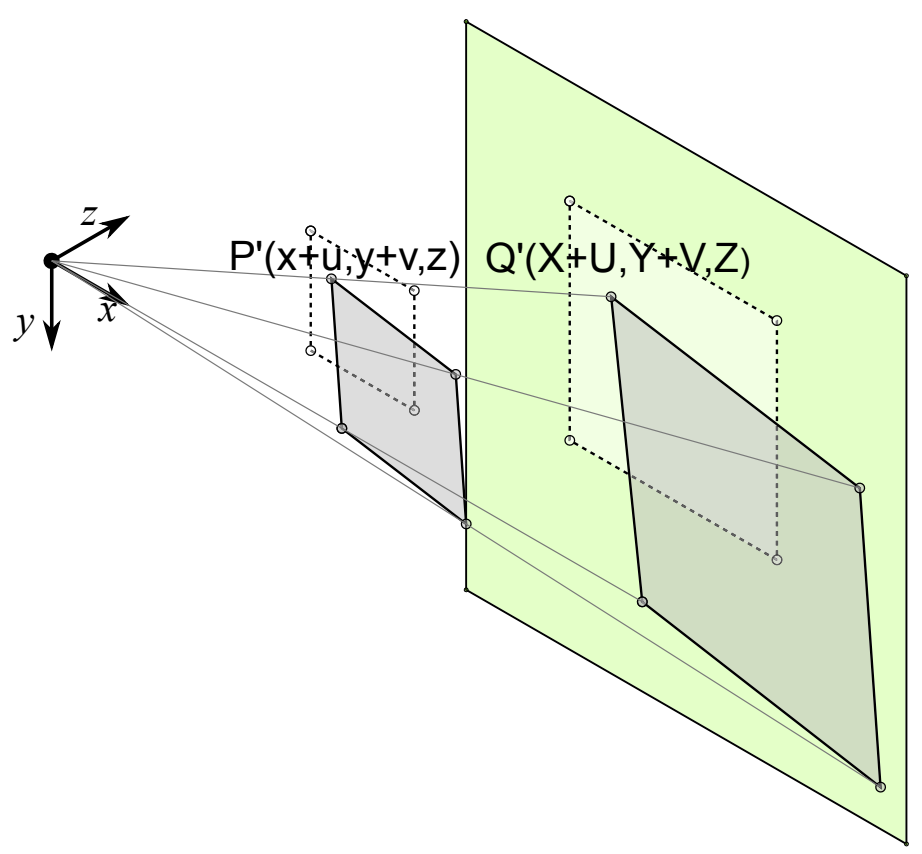

Figure 12: Magnification of an element after in-plane strain

$$
\begin{array}{r}
\frac{\partial U}{\partial Y}=\frac{\partial U}{\partial y} \frac{\partial y}{\partial Y}=\left(\frac{\partial u}{\partial y} \frac{Z}{z}\right)\left(\frac{z}{Z}\right)=\frac{\partial u}{\partial y} \\
\frac{\partial V}{\partial X}=\frac{\partial v}{\partial x} \\
\left(1+\frac{\partial U}{\partial X}\right) d X=\left(1+\frac{\partial u}{\partial x}\right) d x \\
\left(1+\frac{\partial V}{\partial Y}\right) d Y=\left(1+\frac{\partial v}{\partial y}\right) d y
\end{array}
$$

The equations derived above show the linear relationship between relative object placement and image size. Due to the projection magnification, the relationship between actual and perceived object displacements has a scaling factor of $\frac{Z}{Z}$, 
yet the strains remain equivalent.

$$
\begin{aligned}
& \varepsilon_{x x}=\varepsilon_{X X} \\
& \varepsilon_{y y}=\varepsilon_{Y Y} \\
& \varepsilon_{x y}=\varepsilon_{X Y} \\
& \varepsilon_{y x}=\varepsilon_{Y X}
\end{aligned}
$$

The equations developed to show the effect of magnification on perceived object size, displacements and strains were developped assuming a point-source focal spot. In practice, the focal spot has finite size, measured as per International Electrotechnical Commission (IEC) standard 60336 [16]. The nominal focal spot size, specified by the manufacturer corresponds to the size of the region on the anode covered by the incident electron beam. X-ray photons will have a slightly different trajectory to a specific point on the object based on their location of origin on the focal spot. Fig. 13 demonstrates the different projections of the element on the image plane from $X$-rays originating at each of the four corners of the focal spot. 

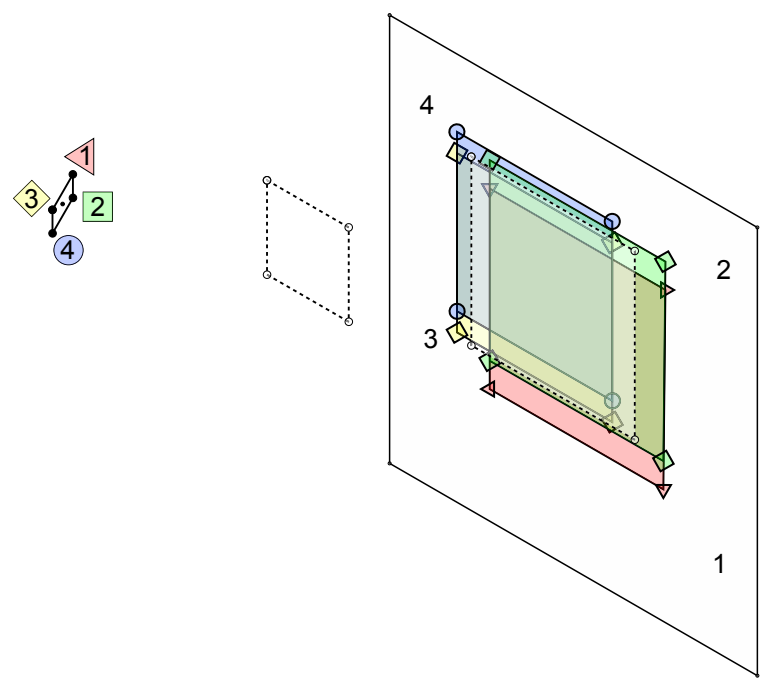

Figure 13: Magnification element from four points on the focal spot.

The Fig. 13 demonstrates the effect of a finite focal spot on object projection. The object size and placement relative to the focal spot in Fig. 13 are not representative of a realistic system as the focal spot is significantly larger than expected in practice to emphasize edge effects; however, it is important to consider different possible projections and their effect on image quality. The image will include contributions from all photons originating on the focal spot, regardless of their specific point of origin. The net effect of slight variations in photon trajectory result in blurring of feature edges (unsharpness) on the image [17]. In practice, electrons incident on the focal spot are normally distributed around its centre, resulting in the majority of X-ray photons following trajectories similar to that of an optimal point source [9]. Although the blur profile is tube dependent, a Gaussian distribution of electrons on the focal spot results in a sigmoid edge blur [7]. The magnitude and shape of edge blur is dependent on the location of the object relative to the focal spot, as shown in Fig. 14.

As shown in Fig. 13 and Fig. 14, the same physical feature can appear at different locations based on the focal spot size and location of the imaging feature 
(a)
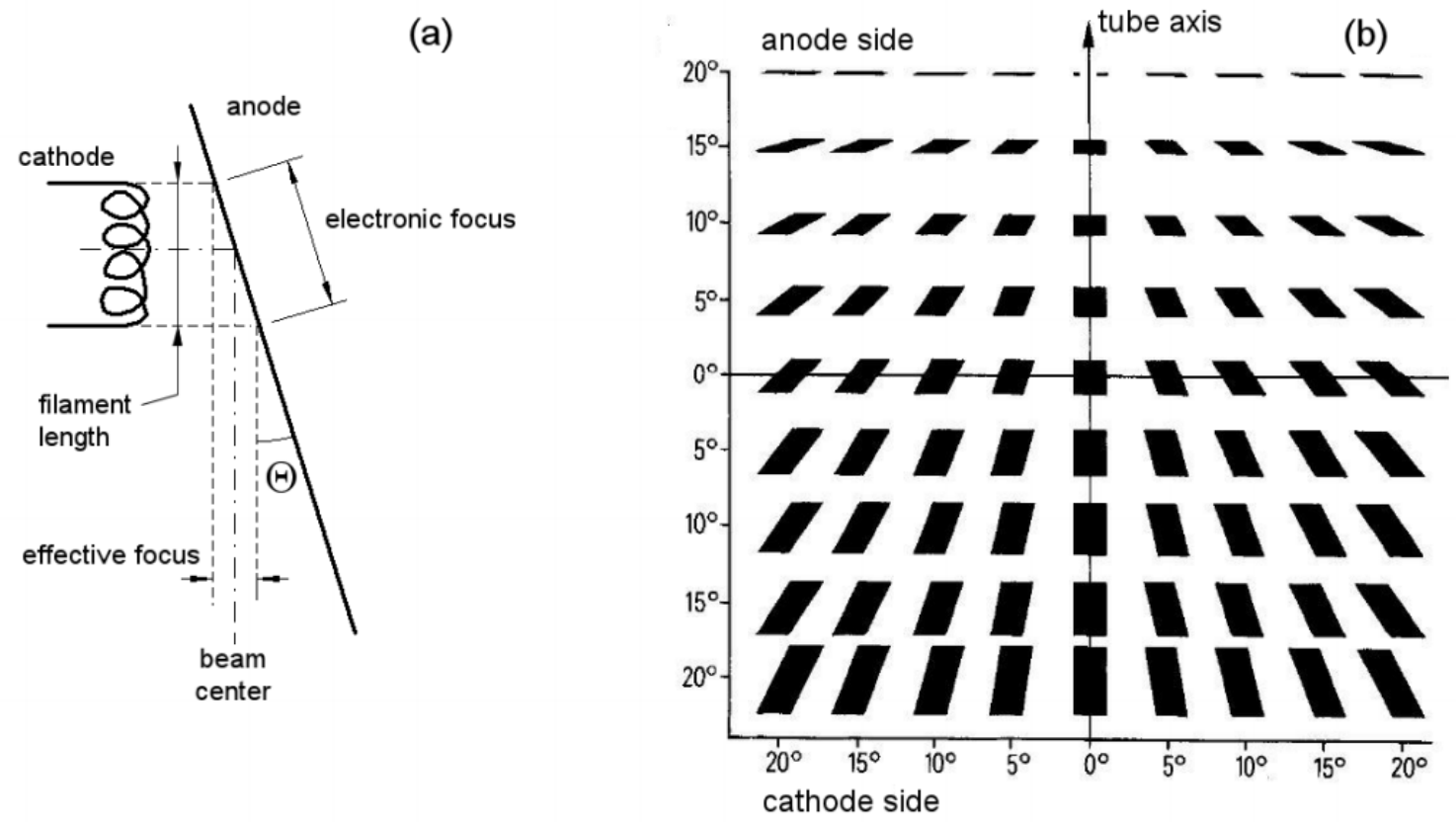

Figure 14: Visualization of edge blur variation through the field of view, OIAEA [18].

relative to the generator and the image acquisition system. An additional geometric consideration is the linear magnification of blur due to location of a feature relative to the imaging sensor caused by the conical shape of the X-ray distribution. A feature imaged close to the sensor and far from the source will have less magnification and sharper detail than when placed closer to the source [7], [2] .

\subsection{X-ray Photon-Material Interaction}

As $\mathrm{X}$-rays pass through a material, there is a potential for interaction, with the probability of photon-material interactions decreasing as the photon energy increases. There are four possible types of X-ray interaction with material, Rayleigh scatter, Compton scatter, photoelectric effect, and pair production. A brief description of these processes is provided here; however, an in-depth physical explanation is provided in Appendix A. 
Rayleigh scattering occurs when an incident $X$-ray wave interacts with a whole atom. X-ray absorption causes synchronized electron vibration due to the oscillating electromagnetic wave. This vibration results in instantaneous re-emission of the photon at the same energy level, but on a deviated trajectory [2].

Compton scatter involves a collision between a photon and an electron. The photon will transfer a portion of its energy to the electron, resulting in ionization of the electron. The electron is ejected with additional kinetic energy. As only a portion of the photon energy is transferred to the electron, the scattered X-ray will have a higher wavelength (i.e., lower energy) and a deviated trajectory [2] . Both Compton and Rayleigh scatter are detrimental to image quality due to photons reaching the sensor via non-linear pathways, contributing to image noise [19] .

Similarly to Compton scatter, the photoelectric effect describes interactions between X-ray photons and electrons. Here, a photon loses all of its energy after interacting with an electron. The photon is absorbed and the energy is first used to overcome the energy binding the electron to the nucleus, with the balance being transformed to kinetic energy. The photoelectric effect is not detrimental to image quality as interacting photons are completely absorbed and thus do not reach the sensor [2].

Pair production involves a photon-atom interaction in which sufficient energy is transferred to create an electron-positron pair. However, from $E=m c^{2}$, we know this cannot occur below energy levels of $1.02 \mathrm{MeV}$, well above the power levels that can be achieved using a rotating anode generator [2].

Understanding photon-matter interactions is critical to maintaining sufficient image quality. For example, scattered photons will reduce image quality by incorrectly representing the the $2 \mathrm{D}$ projection of a feature. As scattered photons can be detrimental to image quality, methods such as incorporation of an anti-scatter grid 
can be used to preferentially attenuate photons deviated from their original trajectories as shown in Fig. 15. Although an anti-scatter grid will result in a darker image as less photons are reaching the image acquisition system, the recorded information contains less noise.

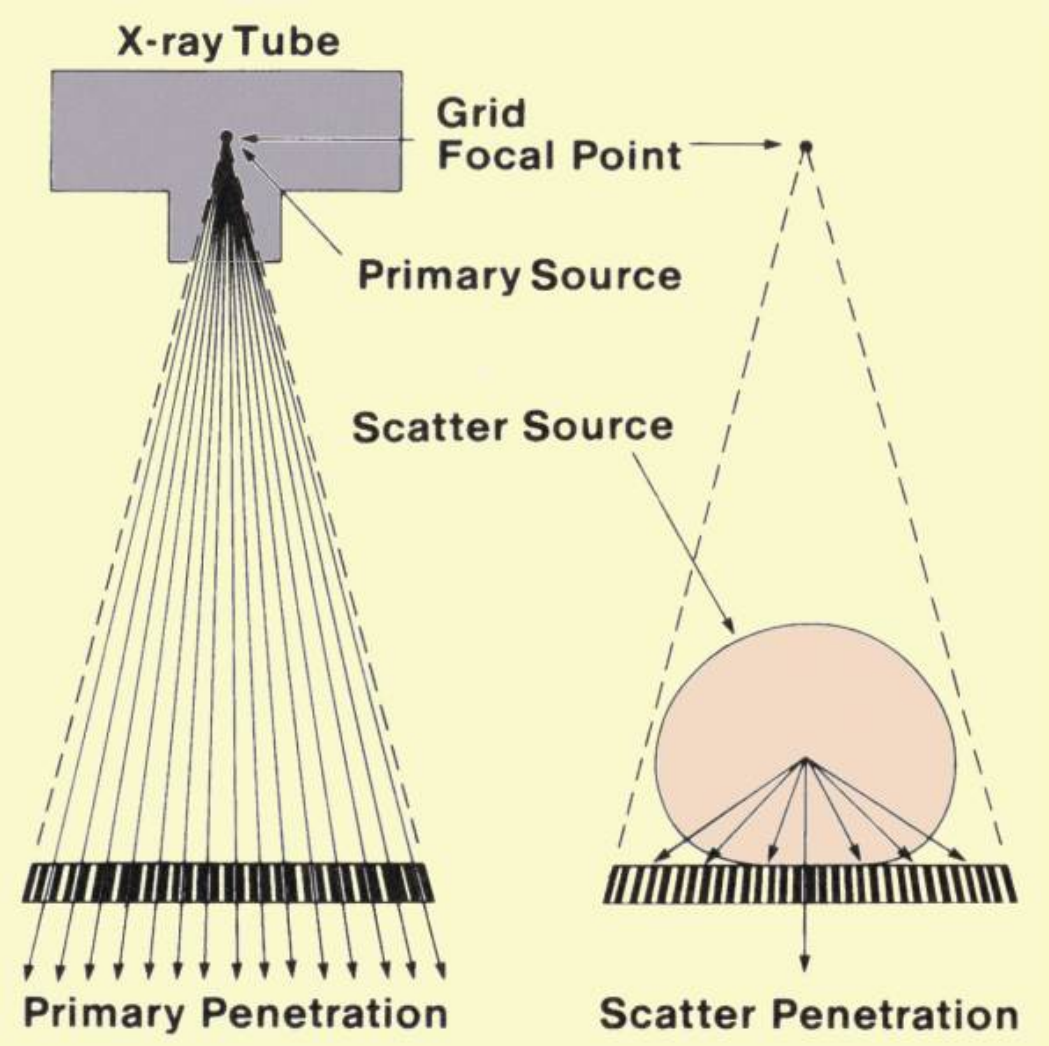

Figure 15: Anti scatter grid preventing scattered photons from reaching the sensor plane, increasing image quality [9]. 


\subsubsection{Attenuation Coefficients}

A photon incident on a material is either attenuated (ie. scattered or absorbed) or transmitted through the material. The potential for material interactions is quantified using attenuation coefficients. The mass attenuation coefficient has contributions from Compton and Rayleigh scatter, the photoelectric effect and pair production [2].

$$
\mu / \rho=\mu / \rho_{\text {Rayleigh }}+\mu / \rho_{\text {Compton }}+\mu / \rho_{\text {photoelectric }}+\mu / \rho_{\text {pairproduction }}
$$

The mass attenuation represents the quantity of photons that are scattered or absorbed as the beam passes through a material. The probability of interaction is dependent on the cross-sectional area density (i.e., relative 2D area occupied by atoms) [2]. The National Institue of Standards and Technology (NIST) published an open-access X-ray attenuation profile database (XCOM) for the first 100 elements for energy levels of $1 \mathrm{keV}$ to $100 \mathrm{GeV}$ [20].

A monocromatic X-ray beam with an initial photon fluence, $N_{0}$, is attenuated as it penetrates a material, resulting in a lower transmitted photon fluence of $N$ (Figure 16). The fraction of photons transmitted through the material is related to the penetration thickness, $t$, material density, $\rho$, and mass attenuation coefficient, $\mu / \rho$, via the Beer-Lambert Law (Eq. 34) [2]

$$
N=N_{0} e^{-(\mu / \rho) \rho t}
$$

Therefore, of two materials with similar mass attenuation coefficients, the higher density material will have greater X-ray attenuation. The exponential decay 


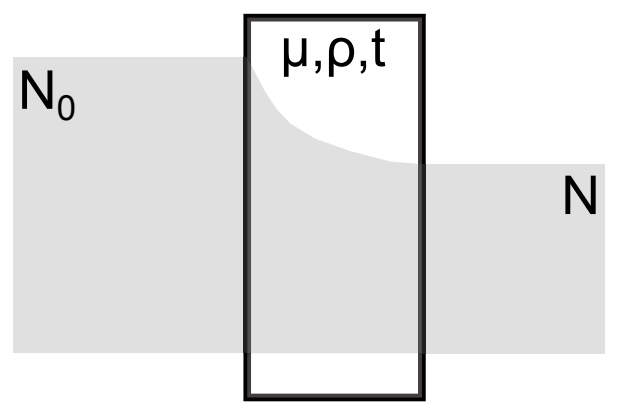

Figure 16: Illustration of $X$-ray photon attenuation.

(Eq. 34) can also be written in the following form [2]:

$$
N=N_{0} e^{-\mu t}
$$

where $\mu$ is now the linear attenuation coefficient. Additionally, Eq. 35 can be extended for the case where the specimen is composed of $i$ layers of different materials as shown in Fig. 17, resulting in Eq. 36.

$$
N=N_{0} e^{-\mu_{1} t_{1}} e^{-\mu_{2} t_{2}} \ldots e^{-\mu_{i} t_{i}}=N_{0} e^{-\sum_{i} \mu_{i} t_{i}}
$$

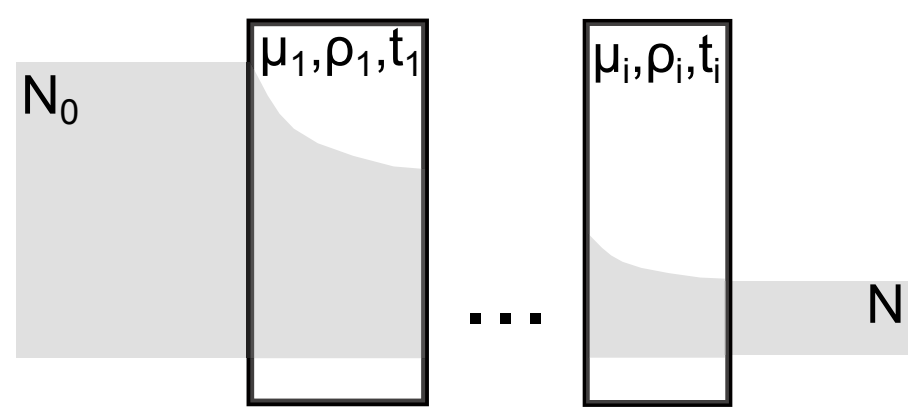

Figure 17: Illustration of $X$-ray photon attenuation through multiple layers of material.

Although it is possible to express the transmission levels in number of photons (i.e., $N$ ), it is more commonly expressed as a total energy, or the product of photon 
count and specific energy [3]:

$$
E_{\text {tot }}=E \cdot N(E)
$$

As photons are attenuated by the various types of scatter, a fraction of their energy is absorbed by the material, while the remaining energy has contributions from deviated (i.e.,scattered) and unscattered photons. The mass energy absorption coefficient, $(\mu / \rho)_{e n}$, represents the fraction of the attenuated energy absorbed by the material, including all the energy from the photoelectric effect, and the fraction of energy transferred to electrons in primary Compton scatter as shown in Eq. 38 [21].

$$
\frac{E_{a b}}{E_{a t}}=\frac{\mu / \rho_{e n}}{\mu / \rho}
$$

\subsubsection{Spectral Material Attenuation Response}

Eq. 36, describing the exponential decay of photon fluence was derived for a monochromatic beam of energy $E$. When the incident beam is a spectrum, Eq. 36 must be expanded to consider the attenuation coefficient for all photon energy levels [2]. The spectral fluence, in photons $/ \mathrm{mm}^{2}$, is defined as $\Phi(E)$.

$$
\Phi(E)=\sum_{E} \Phi_{0}(E) e^{-\sum_{i} \mu_{i, E} t_{i}}
$$

The total photon energy in $\mathrm{keV} / \mathrm{mm}^{2}, \Psi(E)$, must now consider photon quantity across all energy levels, modifying Eq. 37 to 40 .

$$
E_{\text {tot }}=\sum_{E} \Psi(E)=\sum_{E} \Phi(E) E
$$

The incident and transmitted energies are represented by the area under the photon-fluence versus power spectrum curve. A simulated of a $100 \mathrm{kV}_{p}$ and 1 
mGy KERMA incident spectrum and the resulting transmission through $2.5 \mathrm{~mm}$ of aluminum are shown in Fig. 18. Kinetic EneRgy per MAss of air (KERMA) is a measure of $\mathrm{X}$-ray exposure. A change in incident KERMA is equivalent to scaling the number of photons in the spectrum [22]. In air, the KERMA is expressed as the following, with energy bins of $1 \mathrm{keV}$ [2]:

$$
K E R M A \approx k \sum_{E} \Phi(E) \frac{\mu(E)}{\rho} E
$$

where the constant, $k$, is equal to $1.6021 \times 10^{-} 13$ (Gray/keV). KERMA is a measure of radiation exposure which is inversely proportional to distance squared:

$$
\frac{K E R M A_{1}}{Z_{1}^{2}}=\frac{K E R M A_{2}}{Z_{2}^{2}}
$$

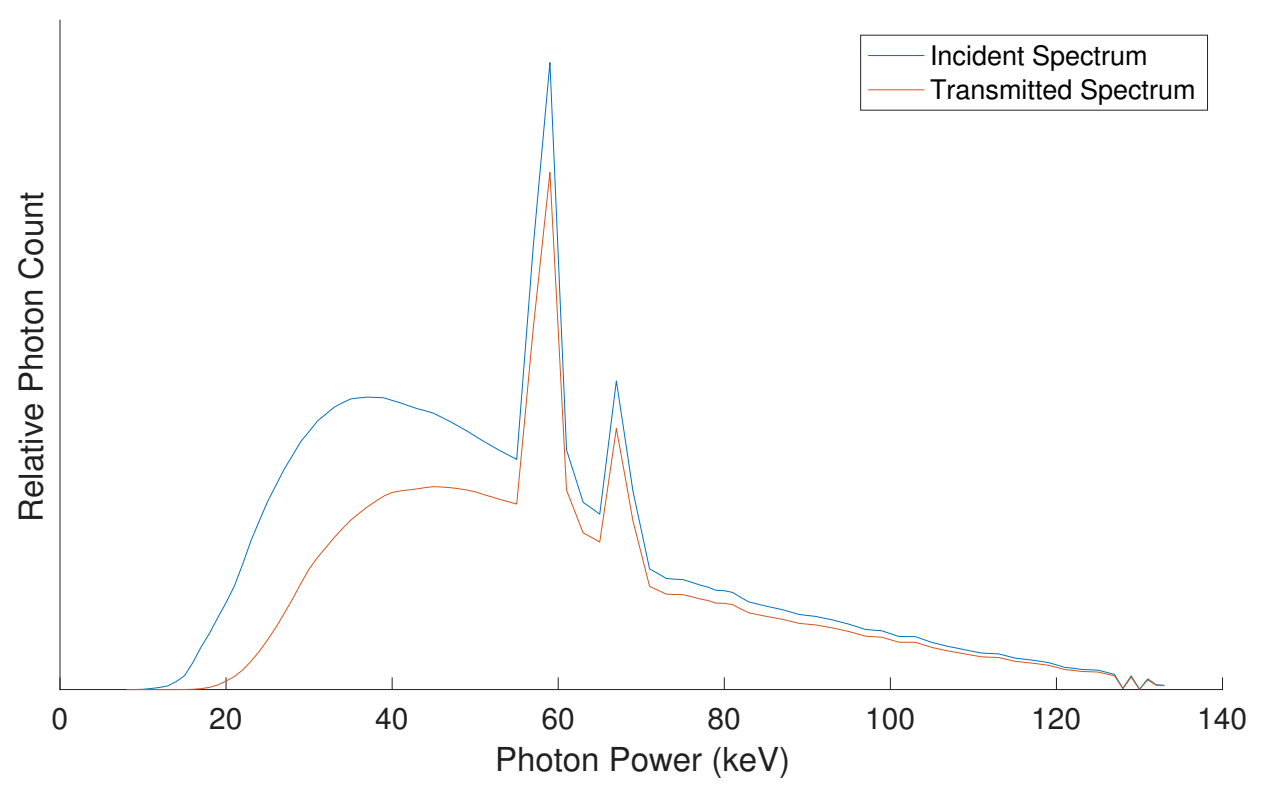

Figure 18: Incident spectrum and transmitted spectrum through $2.5 \mathrm{~mm}$ aluminum at $140 \mathrm{kV}_{p}$ and $1 \mathrm{mGy}$ KERMA, data from [13].

As shown in Fig. 18, lower power photons are more likely to interact with 
atoms, resulting in a greater loss of energy at the lower end of the spectrum. Based on Eq. 40, the perceived contrast in transmitted energy between the two spectra shown in Fig. 18 is the area between the two curves.

\subsection{X-ray Contrast}

Contrast created by two identical parallel X-ray beams having different paths through a specimen is shown in Fig 19. The quantity of X-rays either transmitted through the bulk specimen, $\left(\mathrm{N}_{s p}\right)$, or along a path containing a radio-contrast marker, $\left(\mathrm{N}_{m a}\right)$, will be different.

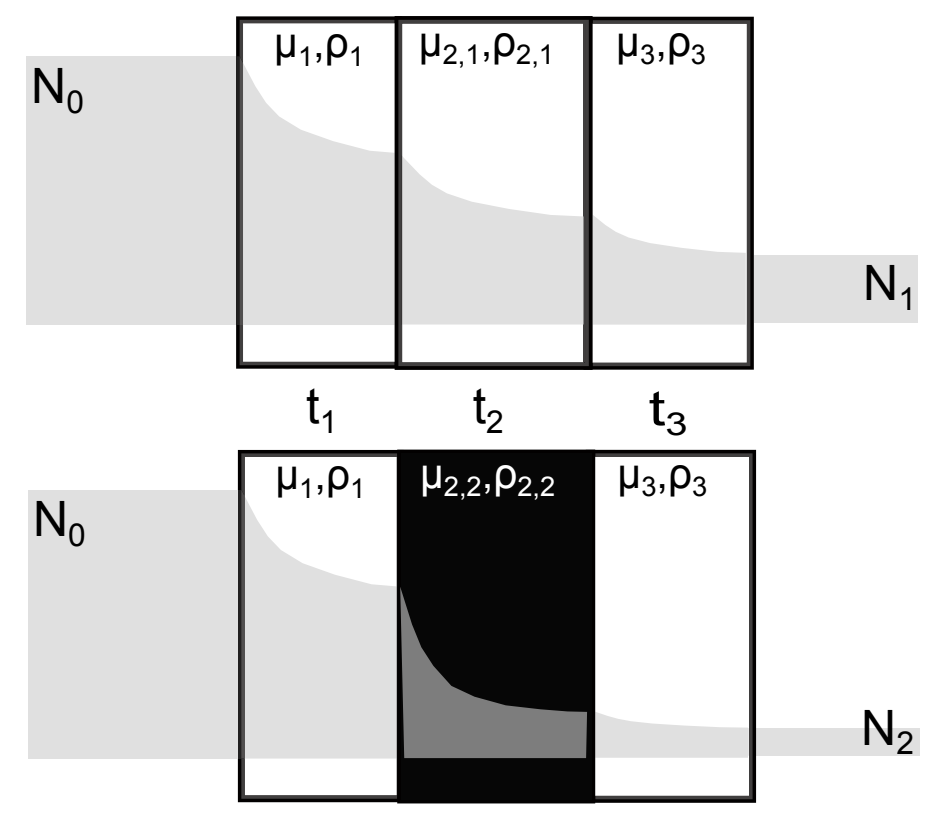

Figure 19: X-ray transmission variation through two different cross-sectional paths.

The bulk specimen and marker are composed of different materials having different attenuation profiles. The radio-contrast marker can either have a higher or lower attenuation profile than the bulk medium, however we will discuss the case where it has increased attenuation. The energy contrast at the sensor between the 
two paths (specimen with $i$ layers and markers with $j$ layers) is presented below in terms of both transmitted and initial photon counts [3].

$$
\begin{array}{r}
C=\sum_{E} \Psi_{s p}(E)-\Psi_{m a}(E)=\sum_{E} \Phi_{s p}(E) E-\Phi_{m a}(E) E \\
C=\sum_{E} \Phi_{0}(E) E\left(e^{-\sum_{i} \mu_{i}(E) t_{i}}-e^{-\sum_{j} \mu_{j}(E) t_{j}}\right)
\end{array}
$$

\subsection{X-ray Detection}

Methods of measuring electromagnetic waves vary by wavelength. Two main types of X-ray detectors are used, where either spatial information is conserved, or cumulative exposure is measured. The former, used for imaging, will be discussed here; the latter, used to quantify exposure dose, relies on similar principles will not be addressed as it cannot be used for imaging.

The fundamental concept used in X-ray sensors is scintillation, a process by which a material emits light as a result of absorbed radiation [23]. Depending on the application, organic or inorganic scintillators are used, each producing a characteristic output spectrum, with different conversion efficiencies (photons/keV), and decay times [23].

The luminescence produced by a scintillating material is captured using ana$\log$ or digital techniques. For dynamic events, several flash X-ray generators are configured in 3D space to record a single frame each from several angles with a small delay between exposures. Image registration is performed using fixed fiducial markers, allowing for deformation comparison between images [24]. Alternatively, a rotating anode generator can be used to produce X-ray continuously over longer periods of time.

Current $\mathrm{X}$-ray technologies capture images digitally using flat-panel detectors, 
generally composed of a scintillator and digital sensor. The digital sensor is coupled to optical sensors, commonly either a charge-coupled device (CCD), or a complementary metal-oxide-semiconductors (CMOS), [23]. As these are digital sensors, continuous recordings are possible. Commercial X-ray sensor panels are designed and built primarily for medical applications and their specifications generally match those of typical diagnostic imaging tests.

Commercial sensor panels are insufficient for certain applications such as studying dynamic impacts of internal planes as the frame rates required to study high-speed dynamic events cannot be achieved using commercially available systems. As most uses are medical, minimization of radiation dose absorbed by the patient is critical. For example, dynamic radiography frame rates are typically 2530 fps for fluoroscopy [25].

Uncoupling the photon conversion and image acquisition phases allow for the construction of systems able to achieve significantly higher recording rates. Commercially available low-persistence scintillators and high-speed cameras can therefore be sourced to meet design requirements.

The light output of a scintillator is directly proportional to the absorbed radiation energy. Eq. 39 described the anticipated fraction of photons transmitted through a material. Therefore, the fraction of photons either scattered or absorbed by the scintillator of thickness, $t_{s c}$, and mass attenuation coefficient $\mu / \rho_{s c}(E)$ is

$$
\Phi_{s c}(E)=\sum_{E} \Phi_{0}(E)\left(e^{-\sum_{i} \mu_{i}(E) t_{i}}\right)\left(1-e^{-\mu_{s c}(E) t_{s c}}\right)
$$

however, as per Eq. 38, only a portion of scattered photon energy is absorbed by the scintillator. The spectral absorbed energy signal becomes the following:

$$
\Psi_{a b}(E)=\sum_{E} \Phi_{0}(E) E\left(e^{-\sum_{i} \mu_{i}(E) t_{i}}\right)\left(1-e^{-\mu_{s c}(E) t_{s c}}\right) \frac{\mu / \rho_{s c, e n}(E)}{\mu / \rho_{s c}(E)}
$$


The contrast of energy absorbed by the scintillator therefore becomes:

$$
C_{a b}=\sum_{E} \Phi_{0}(E) E\left(e^{-\sum_{i} \mu_{i}(E) t_{i}}-e^{-\sum_{j} \mu_{j}(E) t_{j}}\right)\left(1-e^{-\mu_{s c}(E) t_{s c}}\right) \frac{\mu / \rho_{s c, e n}(E)}{\mu / \rho_{s c}(E)}
$$

From Eq. 47, the contrast will increase with the scintillator thickness, $t_{s c}$. In practice this is not an ideal solution. As scintillator thickness increases, more Xray photons are absorbed, therefore more visible light is emitted; however, spatial resolution decreases because the light will scatter before reaching the surface of the scintillator. A reflective back layer can be added to the scintillator surface closest to the source, directing more light towards the sensor, yet this method also decreases spatial resolution. The effects of scintillator thickness and adding a back layer are shown in Fig. 20 [2].

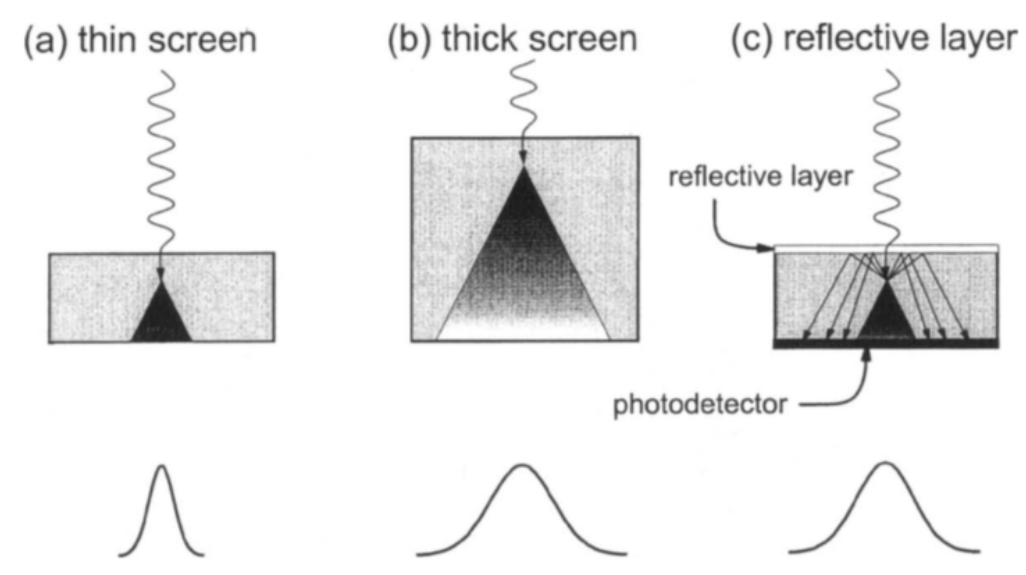

Figure 20: Effect of scintillator thickness and reflective back layer on perceived signal at scintillator surface [2].

The spatial resolution of an X-ray system is quantified using the modultation transfer function (MTF), a measure of minimum perceived variation in an image [26]. An X-ray image of a lead phantom used to measure distinguishable line-pairs 


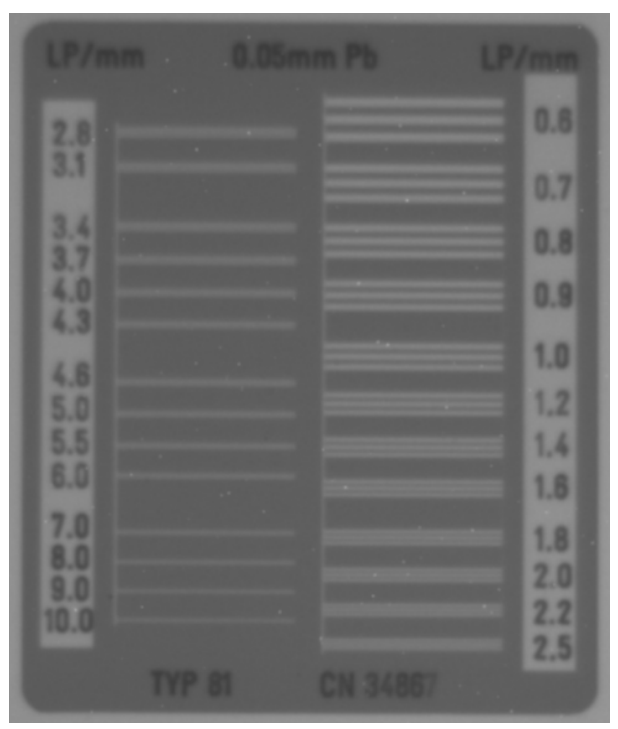

Figure 21: X-ray resolution test pattern (Leeds Test Object, North Yorkshire, UK), used to quantify system spatial resolution in line-pairs per $\mathrm{mm}$.

per $\mathrm{mm}$ is shown in Fig. 21. Here, the resolution distinguishable is $\approx 1.8-2 \mathrm{LP} / \mathrm{mm}$.

The contrast transfer function (CTF), the ability to distinguish square waves, is directly measured using line pairs per milimeter; however, the MTF is the ability to measure sinusoidal waves, and thus cannot be directly determined using a standard image phantom. An increase in scintillator thickness lowers the CTF and MTF of an imaging system, therefore the balance between spatial resolution and X-ray stopping power (i.e., light output) must be considered [26].

In our application, the signal provided in the scintillator is captured using a high-speed camera. Therefore, all aspects from energy absorbed by the scintillator to image pixel value are considered to be part of the image acquisition system. The optical system gain, $G$, of this system is described by the following equation, including gain contributions from the scintillator, optics, and camera:

$$
G=g_{s c} g_{o p t} g_{c a m}=g_{s c}\left(\sum_{E} \frac{N(E)}{\sum_{E} N(E)} \frac{\Omega}{4 \pi} g_{m i}(E) g_{l e}(E) F_{F} Q E(E)\right) g_{c a m}
$$


where the scintillator conversion gain, $g_{s c}$, is measured in photons emitted per absorbed energy (photons $/ \mathrm{keV}$ ), $N(E) / \sum_{E} N(E)$ is the normalized scintillator emission spectrum of the scintillator, the spectral mirror reflectivity gain, $g_{m i}(E)$, the light reaching each sensor pixel, $\frac{\Omega}{4 \pi}$ (in steradians), the camera fill factor, or sensor area/total area, $F_{F}$, sensor quantum efficiency, $Q E(E)$, and camera gain $g_{c a m}$. These values can either be determined from manufacturer specifications, of approximated using a combination of available data and experimental calibration. The signal, read as pixel values is

$$
S=G \sum_{E} \Phi_{0}(E) E\left(e^{-\sum_{i} \mu_{i}(E) t_{i}}\right)\left(1-e^{-\mu_{s c}(E) t_{s c}}\right) \frac{\left(\mu / \rho_{s c, e n}(E)\right.}{\mu / \rho_{s c}(E)}
$$

with corresponding contrast:

$$
C=G \sum_{E} E \Phi_{0}(E)\left(e^{-\sum_{i} \mu_{i}(E) t_{i}}-e^{-\sum_{j} \mu_{j}(E) t_{j}}\right)\left(1-e^{-\mu_{s c}(E) t_{s c}}\right) \frac{\mu / \rho_{s c, e n}(E)}{\mu / \rho_{s c}(E)}
$$

\subsection{X-ray Noise}

Optical DIC implements two methods to ensure high image quality: high contrast speckles (black on white), and increasing ambient light as needed to increase photon flux even with very short exposure times. Both of these methods ensure high image contrast. Increasing radiographic photon flux or peak voltage to increase brightness and contrast is not always feasible due to focal spot limitations. Therefore, images in high-speed analyses will appear darker if the specimen has high X-ray attenuation or thick penetration depth. For this reason, the contrast-to-noise ratio (CNR) cannot be neglected.

The broad definition of CNR that will be developed in this chapter relates the 
difference in two signals, $\Delta S$, to the background noise power spectrum, $N P S_{b g}$ [26].

$$
C N R=\frac{\Delta S}{N P S}=\frac{\left|S-S_{b g}\right|}{\sqrt{N P S_{b g}}}
$$

The background signal is defined as penetrating through the specimen alone $\left(S_{s p}\right)$ while the second signal passes through the specimen and contrast markers $\left(S_{m a}\right)$. Eq. 51 is then written as:

$$
C N R=\frac{S_{s p}-S_{m a}}{\sqrt{N P S_{s p}}}
$$

The signals are determined using Eq. 49, while the noise power spectrum will be determined by describing the variance in processes from photon generation to pixel value. X-ray photon production is considered to be a Poisson random process, therefore, the variance in photons is the same as the signal [26]:

$$
\sigma_{\text {poisson }}^{2}=N
$$

NPS is defined in the frequency domain; however, as the X-ray distribution is uniform and uncorrelated, only the zero-frequency contributions are considered. For this special case, the photon distribution has variance equivalent to the noise power spectrum [26]:

$$
N P S=\sigma^{2}
$$

The variance in photons incident on the scintillator, $\sigma_{s c}^{2}$, should be expressed as a function of absorbed energy. The number of photons incident on the scintillator (Eq. 45) follows a Poisson distribution with variance equal to the mean expected value (Eq. 53). The multiplicative terms in Eq. 46 are constant scalar terms for each 
energy level resulting in mono-wavelength absorbed energy variance of:

$$
\sigma_{a b}^{2}(E)=\sigma_{s c}^{2}(E)\left(E_{a b} \frac{(\mu / \rho)_{s c, e n}(E)}{(\mu / \rho)_{s c}(E)}\right)^{2}
$$

where the total energy variance is the sum over all wavelengths:

$$
\sigma_{a b}^{2}=\sum_{E} \sigma_{a b}^{2}(E)
$$

The noise power spectrum of energy deposited on the scintillator is then:

$$
N P S_{a b}=\sigma_{a b}^{2}
$$

The next stage in the image acquisition system is photon conversion where lower energy photons are emitted, resulting in quantum amplification. The conversion efficiency, $g_{s c}$, is expressed in number of photons produced per unit energy deposited. This process is also a Poisson random process, with mean and variance of $g_{s c}$ [3]. The cascading of two Poisson random processes must be considered in the next step of the NPS. Generally, a quantum amplification stage has the following effect on noise power spectrum:

$$
N P S_{\text {out }}=g^{2} N P S_{\text {in }}+S_{\text {in }} \sigma_{g}^{2}
$$

where $S_{i n}$ is the incident signal the quantum amplification has mean gain $\bar{g}$, and variance $\sigma_{g}^{2}$. The noise power spectrum of photons emitted by the scintillator becomes [26] :

$$
N P S_{e m}=g_{s c}^{2} \sigma_{a b}^{2}+E_{a b} g_{s c}
$$

Photons created in the scintillator have a quantum amplification dependent on 
MTF:

$$
N P S_{\text {out }}=\left(N P S_{\text {in }}-S_{\text {in }}\right) M T F^{2}+S_{\text {in }} \approx N P S_{\text {in }}
$$

As the markers size used in experiments will be significantly larger than the resolution limit of the system, the MTF term can be approximated using low-order terms and effectively assumed to be unity.

Next, the quantity of light reaching the sensor is required. For every photon created at the scintillator, there is a probability that an electron will be freed in the camera sensor. These are processes with an associated probability of reaching the sensor (solid angle), being reflected by the mirror, transmitted by the lens, and converted to electrons (quantum efficiency). Each is a binomial process with a probability of occurrence between 0 and 1 [26]. The spectral response must also be considered, including the contribution from the scintillator output. Therefore, the probability that a photon created at the scintillator will reach the sensor and be converted to an electron is:

$$
g_{\text {opt }}=\sum_{E} \frac{N(E)}{\sum_{E} N(E)} \frac{\Omega}{4 \pi} g_{m i}(E) g_{l e}(E) F_{F} Q E(E)
$$

A binomial process has a variance of [26]:

$$
\sigma_{o p t}^{2}=g_{o p t}\left(1-g_{o p t}\right)
$$

The effect of the binomial optical gain on noise power function of electrons, from Eq. 58 is [26] :

$$
N P S_{e}=g_{o p t}^{2} N P S_{e m}+E_{e m} g_{o p t}\left(1-g_{o p t}\right)=g_{o p t}^{2}\left(N P S_{e m}-E_{e m}\right)+E_{e m} g_{o p t}
$$

Finally, the effect of camera gain from electrons in the sensor to pixel value 
should be evaluated. Noise contributions in the camera include fixed pattern, which is corrected in post-processing, and readout and dark current, which are negligible compared to contributions from photon shot noise in our application. As this is a multiplicative gain with no variance, the pixel-level noise power spectrum becomes:

$$
N P S_{p x}=g_{\text {cam }}^{2} N P S_{e}
$$

The pixel-level contrast-to-noise ratio is then:

$$
C N R_{p x}=\frac{G \sum_{E} \Phi_{0}(E) E\left(e^{\left(-\sum_{i} \mu_{i}(E) t_{i}\right)_{s p}}-e^{\left(-\sum_{j} \mu_{j}(E) t_{j}\right)_{m a}}\right)\left(1-e^{-\mu_{s c}(E) t_{s c}}\right) \frac{\mu_{e n, s c}(E)}{\mu_{s c}(E)}}{\sqrt{g_{c a m}^{2}\left(g_{o p t}^{2}\left(\left(g_{s c}^{2} \sigma_{e n}^{2}+E_{s c} g_{s c}\right)-E_{s c}\right)+E_{s c} g_{o p t}\right)}}
$$

The image-level contrast-to-noise ratio, $C N R_{i}$, is dependent on the number of pixels, $n_{p x}$ spanned by a specific feature (i.e., marker), and the pixel-level $C N R_{p x}$ [3].

$$
C N R_{i}=\sqrt{n_{p x}} C N R_{p x}
$$

Therefore, the image-level CNR is

$$
C N R_{i}=\sqrt{n_{p x}} \cdot \frac{G \sum_{E} \Phi_{0}(E) E\left(e^{\left(-\sum_{i} \mu_{i}(E) t_{i}\right)_{s p}}-e^{\left(-\sum_{j} \mu_{j}(E) t_{j}\right)_{m a}}\right)\left(1-e^{-\mu_{s c}(E) t_{s c}}\right) \frac{\mu_{e n, s c}(E)}{\mu_{s c}(E)}}{\sqrt{\bar{g}_{c a m}^{2}\left(g_{o p t}^{2}\left(\left(\eta_{s c}^{2} \sigma_{e n}^{2}+E_{s c} \eta_{s c}\right)-E_{s c}\right)+E_{s c} g_{o p t}\right)}}
$$

As the number of photons reaching the sensor in high-speed analyses through thick specimens when operating near maximum power cannot be readily increased, there are a limited number of factors that can increase $C N R_{i}$. By default, a large aperture lens should always be used, as it will maximize light incident on the sensor, and a large depth of field is not required to image a flat scintillator.

If the specimen, source-to-scintillator dimensions and camera placement, cannot be modified due to experimental requirements, the only remaining method to 
increase contrast is to optimize the spectral response of all components. Eq. 48 is the product of many factors (scintillator emission, mirror reflectivity, lens transmission, and sensor quantum efficiency. To maximize $G$, components should be selected such that the spectral peaks occur at similar wavelengths.

Several assumptions were required to develop the CNR equations in this section, therefore it should be taken as an idealized upper bound. The reduction in image quality (image noise) from scattered X-ray photons reaching the sensor along a deviated trajectory was not considered. In practice, an anti-scatter grid will significantly reduce this error, however a small contribution may still exist. More complex methods including Monte Carlo simulations could be used to increase the CNR estimate, however this is beyond the scope this work. 


\section{Chapter 3}

\section{Digital Image Correlation}

\subsection{Strain Measurement Techniques}

The relationship between applied load and deformation behaviour of materials is fundamental to the engineering profession. Therefore, many experimental and analytical methods have been developed to measure strain.

A classic example of strain measurement device is the strain gauge transducer. A transducer, such as the one shown in Fig. 22, monitors electrical resistance. Variations in electrical resistance, caused by strain within the transducer wire, are calibrated to provide accurate strain measurements [27]. Although a transducer only provides strain information at a finite point, arrays of transducers in different orientations are used to measure strain at several locations on a specimen.

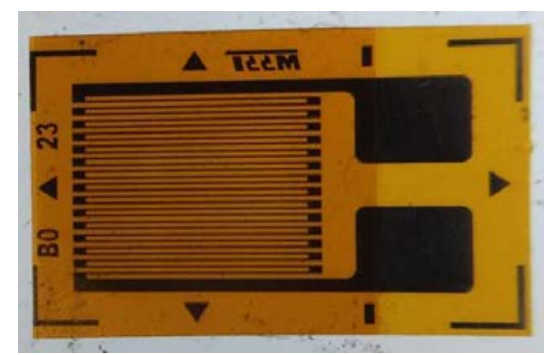

Figure 22: Strain gauge transducer [27]. 
Optical methods including interferometry are widely used for full-field displacement and strain measurements [28]. Interferometry typically uses the superposition of electromagnetic waves following two different paths to the specimen to measure deformation. The phase difference results in either constructive or destructive interference, producing a fringe pattern, an example of which is shown in Fig. 23 [29] .

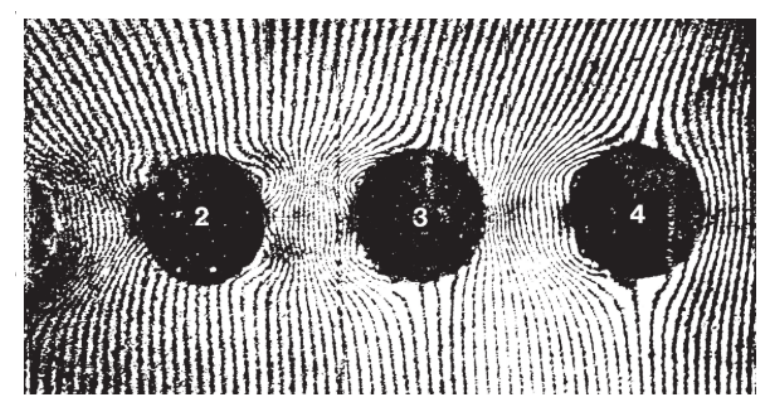

Figure 23: Geometric Moiré fringes created by a shift between a reference and a mobile specimen template. [29].

Geometric Moiré fringes, in Fig. 23, were created by superimposing a fixed master grating and a specimen grating. As the specimen is deformed, alignment of the gratings varies, creating darker and lighter regions. Geometric Moire is one of many methods developed for full field deformation measurement using interferometry [28] .

Most recently, with advances in computer technology and image processing, digital image correlation (DIC) has become an important method for full field displacement and strain measurements [15] . DIC is similar to Particle Image Velocimetry (PIV), where movement of particles allows for flow visualization in transparent fluids. The particles are tracked optically using computer algorithms to determine displacements and velocities. Rather than tracking particles moving in a fluid, whereas DIC tracks changes in the relative position of high-contrast surface finish, for example black speckles on white paint [30]. Fig. 24 shows a speckle 
pattern such as typically seen in DIC.

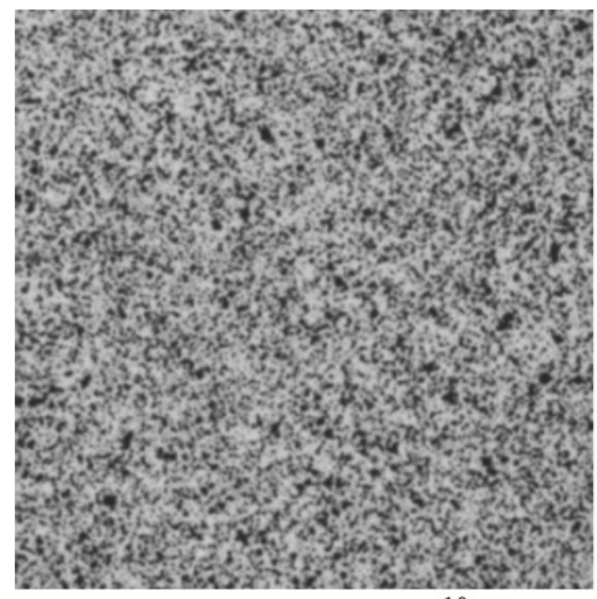

$10 \mathrm{~mm}$

Figure 24: High-contrast painted surface speckle pattern for DIC [15].

\subsection{Digital Image Correlation}

Digital image correlation is a commonly used surface measurement method. It requires monoscopic or stereoscopic image acquisition systems for $2 \mathrm{D}$ or $3 \mathrm{D}$ analyses, respectively [30]. Although there are several published applications and algorithms for 3D DIC, this section will discuss the basics of 2D systems [30]. Digital Volume Correlation (DVC), not to be confused with 3D DIC, is based on tomographic reconstruction of 3D objects [31].

DIC encompasses several algorithm variants used to compute displacement and strain fields in a series of sequential images. As such, DIC itself is independent of image acquisition method, therefore optical, thermal, ultrasound and X-ray have all been used with DIC, see examples in [15], [32], [33], [34]. Analysis rates are limited by the image acquisition system rather than the DIC software. 


\subsubsection{Mathematical Basis}

The foundation of DIC is to determine the transformation between a reference and deformed image(s). The general form of the equations solved by any DIC software are the following [30], [35]:

$$
\begin{aligned}
& x_{i}^{\prime}=x_{i}+u\left(x_{i}, y_{i}\right) \\
& y_{i}^{\prime}=y_{i}+v\left(x_{i}, y_{i}\right)
\end{aligned}
$$

Where the transformation from original points $\mathrm{P}\left(x_{i}, y_{i}\right)$ of a subset centred about $\mathrm{P}_{0}\left(x_{0}, y_{0}\right)$ to $\mathrm{P}^{\prime}\left(x_{i}^{\prime}, y_{i}^{\prime}\right)$ is described by directional shape functions $\xi$ and $\zeta$. In the most basic form, a zero-order shape function is used to describe pure translation as $u$ in the $x$ direction and $v$ in the $y$ direction [30]:

$$
\begin{aligned}
& \xi_{0}\left(x_{i}, y_{i}\right)=u \\
& \zeta_{0}\left(x_{i}, y_{i}\right)=v
\end{aligned}
$$

These equations can only be used for pure translation. The more commonly used shape function formulation includes contributions of first-order displacement gradients $\left(u_{x}, u_{y}, v_{x}, v_{y}\right)$, and position relative to the subset centre $\Delta x=$ $x_{i}-x_{0}$ and $\Delta y=y_{i}-y_{0}[30]$.

$$
\begin{aligned}
& \xi_{1}\left(x_{i}, y_{i}\right)=u(x, y)+u_{x} \Delta x+u_{y} \Delta y \\
& \zeta_{1}\left(x_{i}, y_{i}\right)=v(x, y)+v_{x} \Delta x+v_{y} \Delta y
\end{aligned}
$$


In some cases where large, non-homogeneous deformations occur, secondorder shape functions are used [30].

$$
\begin{gathered}
\xi_{2}\left(x_{i}, y_{i}\right)=u+u_{x} \Delta x+u_{y} \Delta y+\frac{1}{2} u_{x x} \Delta x^{2}+\frac{1}{2} u_{y y} \Delta y^{2}+\frac{1}{2} u_{x y} \Delta x \Delta y \\
\zeta_{2}\left(x_{i}, y_{i}\right)=v+v_{x} \Delta x+v_{y} \Delta y+\frac{1}{2} v_{x x} \Delta x^{2}+\frac{1}{2} v_{y y} \Delta y^{2}+\frac{1}{2} v_{x y} \Delta x \Delta y
\end{gathered}
$$

where the second-order displacement gradients are $u_{x x}, u_{y y}, u_{x y}, v_{x x}, v_{y y}$, and $v_{x y}$.

In practice, image correlation is performed in several steps. First, a pixel-level deformation is determined, followed by a sub-pixel refinement algorithm. The initial estimation is computed using coarse-to-fine or nested-search schemes though frequency domain analyses are also used; correlation after a fast fourier transform (FFT) is significantly less expensive computationally [30]. Certain algorithms are sensitive to small rotation/strain and will not match well in these cases. This is especially true for the FFT as translation is implicitely assumed. Sub-pixel registration is performed using a second algorithm. The pixel-level estimation is required to ensure convergence of the optimization algorithm (typically Newton-Raphson) as it can have a small radius of convergence [36].

Two types of algorithms are used for sub-pixel registration, derived either assuming no deformation of the subset, or including deformation terms in the computation. Coarse-fine search, where the optimal solution is found in a gradually decreasing seach space, and peak finding, where the correlation coefficients are interpolated to determine a maximum are examples of the former [30] . Algorithms implicitely assuming subset deformation are iterative spatial domain cross-correlation (e.g., Newton-Raphson), genetic algorithms and B-spline or finite element-based approaches [30] . Here, discussion will be limited to noting that strain computation occurs either as a final step after deformation fields have been computed, or during the refined registration step. A complete review of both 
algorithm types is presented in [30].

The theoretical derivation of DIC algorithms defines strain as the derivative of displacement (i.e., displacement gradient). In practice, the derivative of the discrete points forming image pixels is unstable. Strain (or, displacement gradient) has high sensitivity to image noise. Therefore, strain fields, are generally computed by first smoothing the displacement field, then performing pixel-wise least-square fit in a local neighbourhood (strain subset) [30].

Although this briefly discusses the algorithms used, the criterion used for matching should also be considered. At each step, correlation is performed by determining an optimal solution that minimizes a cost function. Correlation is performed by evaluating either the the Cross-Correlation $\left(\mathrm{C}_{C C}\right)$ or Sum of Square Differences $\left(C_{S S D}\right)$ criterion [37] .

$$
\begin{array}{r}
C_{C C}=\sum_{i=-M}^{M} \sum_{j=-M}^{M}\left[f\left(x_{i}, y_{j}\right) g\left(x_{i}^{\prime}, y_{j}^{\prime}\right)\right] \\
C_{S S D}=\sum_{i=-M}^{M} \sum_{j=-M}^{M}\left[f\left(x_{i}, y_{j}\right)-g\left(x_{i}^{\prime}, y_{j}^{\prime}\right)\right]^{2}
\end{array}
$$

These correlation coefficients are used to evaluate square subsets measuring $(2 \mathrm{M}+1) \times(2 \mathrm{M}+1)$ with intensity $f$ in the reference image and $g$ in the deformed image [37] .

Various forms of normalized correlation coefficients exist. The zero-normalized formulations $\left(C_{Z N C C}\right.$ and $\left.C_{Z N S S D}\right)$ are invariant to lighting variation between frames and are therefore more robust with noise [37] . 


$$
\begin{array}{r}
C_{Z N C C}=\sum_{i=-M}^{M} \sum_{j=-M}^{M} \frac{\left[f\left(x_{i}, y_{j}\right)-f_{m}\right] \times\left[g\left(x_{i}^{\prime}, y_{j}^{\prime}\right)-g_{m}\right]}{\Delta f \Delta g} \\
C_{Z N S S D}=\sum_{i=-M}^{M} \sum_{j=-M}^{M}\left[\frac{f\left(x_{i}, y_{j}\right)-f_{m}}{\Delta f}-\frac{g\left(x_{i}^{\prime}, y_{j}^{\prime}\right)-g_{m}}{\Delta g}\right]^{2}
\end{array}
$$

The normalization is performed by subtracting the mean subset value, $f_{m}$ and $g_{m}$ defined as the following:

$$
\begin{aligned}
& f_{m}=\frac{1}{(2 M+1)^{2}} \sum_{i=-M}^{M} \sum_{j=-M}^{M} f\left(x_{i}, y_{j}\right) \\
& g_{m}=\frac{1}{(2 M+1)^{2}} \sum_{i=-M}^{M} \sum_{j=-M}^{M} g\left(x_{i}^{\prime}, y_{j}^{\prime}\right)
\end{aligned}
$$

$\Delta f$ and $\Delta g$ are the mean squared difference within a subset, defined as:

$$
\begin{aligned}
\Delta f & =\sqrt{\sum_{i=-M}^{M} \sum_{j=-M}^{M}\left[f\left(x_{i}, y_{j}\right)-f_{m}\right]^{2}} \\
\Delta g & =\sqrt{\sum_{i=-M}^{M} \sum_{j=-M}^{M}\left[g\left(x_{i}^{\prime}, y_{j}^{\prime}\right)-g_{m}\right]^{2}}
\end{aligned}
$$

The two correlation criteria are related by

$$
C_{\text {ZNSSD }}=2\left[1-C_{\text {ZNCC }}\right]
$$

where $C_{Z N S S D}$ is minimized and $C_{Z N C C}$ is maximized for optimal correlation [37], [38]. 
Although there are several DIC algorithm variants and implementation techniques used to improve computational efficiency, this discussion is limited to a preliminary overview of general DIC methods.

\subsubsection{Parameter Space}

Implementations of DIC algorithms require certain assumptions based on practical limitations. The first significant difference between the theoretical derivation and real-world implementation is continuity. Finite image resolution cannot capture the physical speckle continuity. Ideally, each pixel is easily recognized in subsequent images based on its unique, constant intensity value. In practice, the aperture problem whereby multiple features have the same intensity value in the deformed image, leads to several possible matches [30]. Therefore, rather than determining motion by searching for one pixel value, a neighbourhood of surrounding pixels in the reference image is used to identify the location of a specific pixel in the deformed images. The neighbouring pixels form a subset surrounding the pixel of interest. The subset is typically defined as either a square or circle in the reference image, and changes shape, matching the local object deformation [30] .

The subset size is an important parameter in DIC. Selection of an small subset window leads to the correspondence problem, a more general formulation of the aperture problem. The correspondence problem is the failure to identify a unique solution for a reference subset in the deformed image. The correspondence problem occurs independently of subset size in two cases: if the image has periodically repeating features, or insufficient features (i.e., a smooth image) [30]. Therefore, a random speckle pattern is required to avoid the correspondence problem. Furthermore, random speckle patterns allow for the use of smaller subset windows as 
the odds of incorrect matching are significantly lower than in a non-random pattern [39]. To maximize information density, a 50\% area coverage of high contrast (i.e., white on black) should be targeted. Methods including speckle entropy [40] or sum of squares of subset intensity gradients have been proposed for determining optimal subset size based on the speckle pattern; however, it is generally recommended that the subset window should fully encompass at least three speckle features to overcome the convergence problem [30], [37].

Although a large subset window is more unique and more likely to match correctly; however, a first-order deformation function may not be suitable to describe a large subset [30]. An alternative method of reducing solution time is by implementing subset spacing. In this case, the deformation is not solved for at every reference pixel, rather at a set of pixels of uniform spacing throughout the region of interest. For example, a subset spacing of 1 indicates pixels $(1,1),(3,1),(1,3)$, $(3,3)$, etc. would be solved. A subset spacing of 0 is equivalent to solving every pixel. Typically, the subset spacing is significantly smaller than the subset size to ensure no information is lost as data from unsolved pixels is used in several subset windows despite not being solved individually [41].

A similar discussion regarding subset size is required for strain computation. As strain window for displacement differentiation increases, neglected higherorder derivatives may become apparent and cause error as the computed results will be smoothed [39]. Therefore, it is necessary to consider the effects of strain window size on physical accuracy and smoothness. For best results, the strain window will be selected to reflect a known physical deformation field. If the deformation is linear or constant, a large window can be used [30]. 


\subsubsection{Sources of Error}

DIC is a powerful tool that can be implemented in a wide range of scenarios to generate full-field displacement and strain plots. Several commercial and open-source packages are available, making it accessible and relatively easy to use. As colourful, and seemingly meaningful, surface plots can be generated despite improper parameter selection or experimental set-up, it is important to consider possible sources of error in the analysis and their influence on results. Many algorithms achieve sub-pixel accuracy based on their interpolation methods, which implies negligible error; however, this is not feasible unless experimental conditions are carefully controlled and parameters are properly selected. The two most important sources of error in 2D DIC are out-of-plane motion and image quality [30].

\section{Image Quality}

Image quality has been noted by several authors as an important consideration in DIC [42], [43], [44], [45] . Traditional optical DIC typically uses black speckles on a white background to provide a high-contrast surface finish for tracking. Given black and white speckles and enough ambient light, high contrast images can be captured at very high frame rates. Unfortunately, this is not always true for highspeed X-ray image of a thick specimen for two reasons. First, rather than high contrast black on white speckles, lower contrast dark grey speckles on a medium grey background would be expected for example. Second, as it is generally a lowlight application, shot noise from the number of photons reaching the sensor will have relatively higher importance. Shot noise follows a Poisson distribution with variance equal to the expected number of photons (Eq. 53).

The effects of noise were studied by [30] who included a random noise term in the correlation coefficient equation [30]. Under the assumptions that the noise is random with constant mean and independent for each pixel, then the applied and 
measured displacements are mathematically identical only if the gradients caused by speckles are significantly larger than the noise gradients.

The effect of intensity pattern noise was studied in [44]. An analytical model was developed to predict the error on displacement. Several important conclusions were found in this study where the intensity pattern noise was the addition of Gaussian noise of constant mean and variance. The standard deviation of measurement error for displacement 1) decreases as subset size increases, 2) is not sensitive to mean noise, and 3) increases with noise variance.

If noise gradients become important relative to speckle gradients, the implementation of sub-pixel interpolation algorithms becomes challenging as peak locations may vary from frame to frame. Therefore, certain studies recommend smoothing the image before performing DIC analyses [46], and was found to be effective in reducing accuracy for uniform displacement [42]. Although smoothing using a Gaussian kernel was found to be most effective when the standard deviation was between 0.75 and 1.25 for bias error reduction, it is further suggested by [42] that optimal Gaussian smoothing parameters are dependent on the DIC algorithm used.

Although several papers mention a high contrast-to-noise ratio requirement, and effects of image noise on DIC results, no studies specifically quantifying DIC strain error with shot noise were found.

\section{Out-of-Plane Motion}

Out-of-plane motion has contributions from translation and rotation. Analyses for optical DIC consider the effects of object motion on the sensor plane and include a lens in the derivation, as shown in Fig. 25 [15] . 


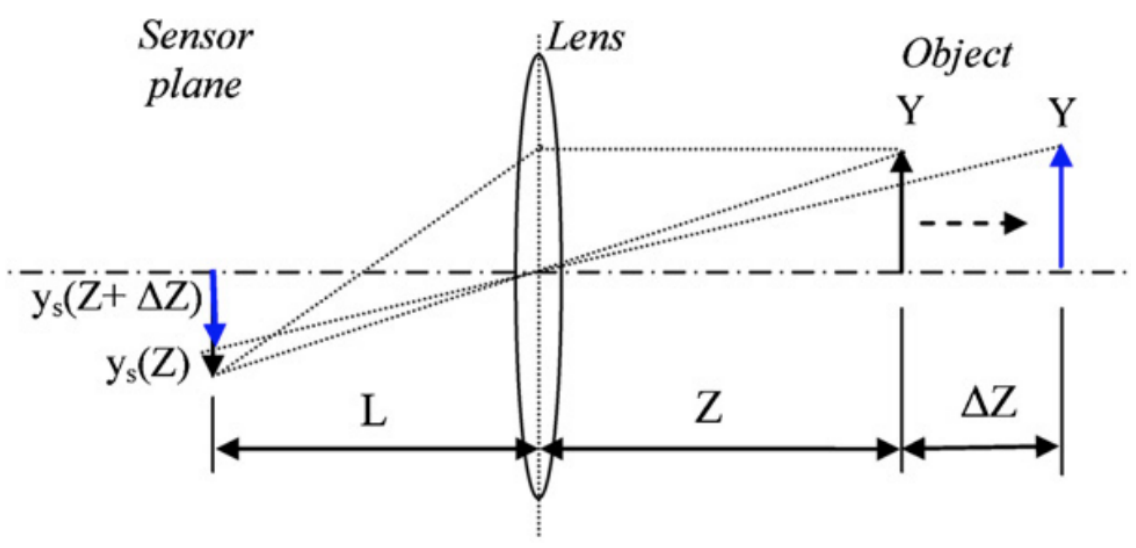

Figure 25: Effect of out-of-plane translation on perceived feature location [15].

Where the reference location of a feature on the sensor is

$$
\begin{aligned}
& x_{s}(Z)=-\frac{L}{Z} X \\
& y_{s}(Z)=-\frac{L}{Z} Y
\end{aligned}
$$

Similarly, the deformed coordinates after out-of-plane motion become [15] :

$$
\begin{gathered}
x_{s}(Z+\Delta Z)=-\frac{L}{Z+\Delta Z} X=-\frac{L}{Z\left(1+\frac{1}{\Delta Z}\right)} X \approx \frac{L X}{Z}\left(1-\frac{\Delta Z}{Z}\right) \\
y_{s}(Z+\Delta Z)=-\frac{L}{Z+\Delta Z} Y=-\frac{L}{Z\left(1+\frac{1}{\Delta Z}\right)} Y \approx-\frac{L Y}{Z}\left(1-\frac{\Delta Z}{Z}\right)
\end{gathered}
$$

Therefore, the perceived displacement caused by out-of-plane motion is

$$
\begin{aligned}
& U(\Delta Z)=x_{s}(Z+\Delta Z)-x_{s}(Z) \approx-\frac{L}{Z} X\left(-\frac{\Delta Z}{Z}\right)=x_{s}\left(-\frac{\Delta Z}{Z}\right) \\
& V(\Delta Z)=y_{s}(Z+\Delta Z)-y_{s}(Z) \approx-\frac{L}{Z} Y\left(-\frac{\Delta Z}{Z}\right)=y_{s}\left(-\frac{\Delta Z}{Z}\right)
\end{aligned}
$$


The strains, calculated as the derivatives of displacements, from [15], are:

$$
\begin{aligned}
& \varepsilon_{x x}=\frac{\partial U(\Delta Z)}{\partial x_{s}} \approx-\frac{\Delta Z}{Z} \\
& \varepsilon_{y y}=\frac{\partial V(\Delta Z)}{\partial x_{s}} \approx-\frac{\Delta Z}{Z}
\end{aligned}
$$

During a standard tensile test for DIC, when a camera is positioned normally to the specimen surface, the Poisson's ratio of the specimen will cause slight outof-plane strain. In practice, the specimen is typically placed sufficiently far from the camera that a small strain in the out-of-plane direction will not influence computed values [30] . For monoscopic image acquisition systems, a telecentric lens, a schematic of which is shown in Fig. 26, is used to greatly reduce error caused by out-of-plane motion [15]. The perceived displacements and strains when using a telecentric lens, where the effective object distance $Z_{e f f}$ is several times larger than $Z$, are

$$
\begin{gathered}
U(\Delta Z) \approx x_{s}\left(-\frac{\Delta Z}{Z_{e f f}}\right) \\
V(\Delta Z) \approx y_{s}\left(-\frac{\Delta Z}{Z_{e f f}}\right) \\
\varepsilon_{x x}=\varepsilon_{y y}=-\frac{\Delta Z}{Z_{e f f}}
\end{gathered}
$$

As $Z_{\text {eff }}>>Z$, errors in both the perceived displacement and strain for a monoscopic system are greatly reduced when using a telecentric lens [15].

A stereoscopic system implements triangulation algorithms to isolate feature location in 3D space. As such, out-of-plane error can be significantly reduced when using stereoscopic image acquisition systems [15]. This method does not require telecentric lenses for out-of-plane motion calibration.

Out-of-plane rotation error, as shown in Fig. 27 is derived following an almost 

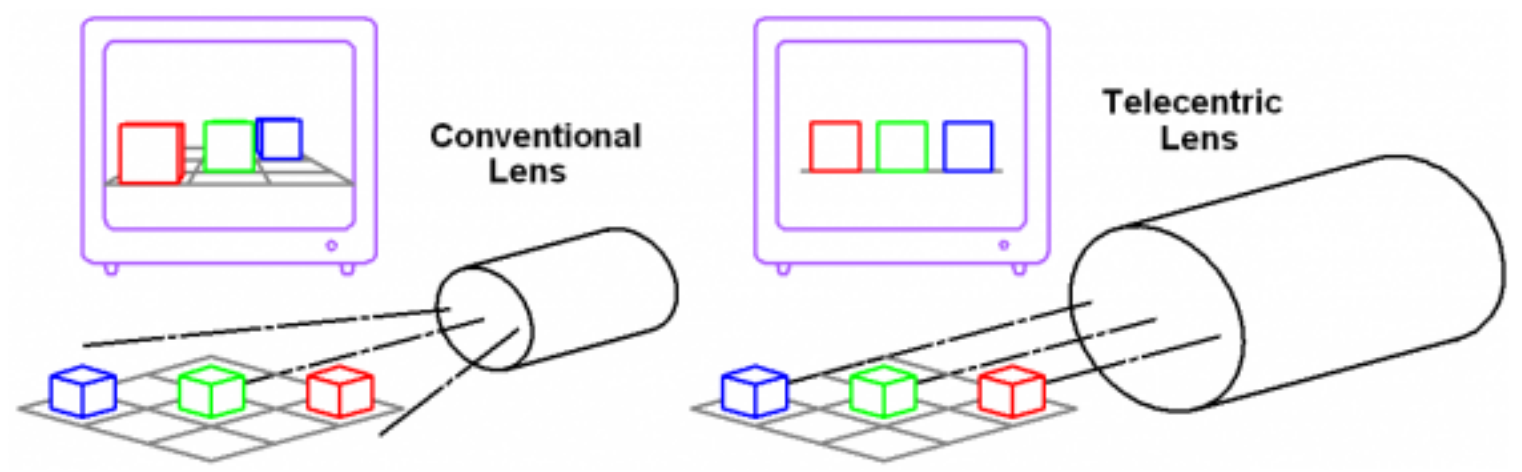

Figure 26: Effect of telecentric lens on perceived image [47].

identical process. The perceived displacements are then [15]:

$$
\begin{array}{r}
U(\Delta Z)=x_{s}(Z+\Delta Z)-x_{s}(Z) \approx-\frac{L}{Z} X\left(-\frac{\Delta Z}{Z}\right)=x_{s}\left(-\frac{Y \sin \theta}{Z}\right) \\
V(\Delta Z)=y_{s}(Z+\Delta Z)-y_{s}(Z) \approx-y_{s}\left(\cos \theta-1-\frac{Y \sin \theta \cos \theta}{Z}\right)
\end{array}
$$

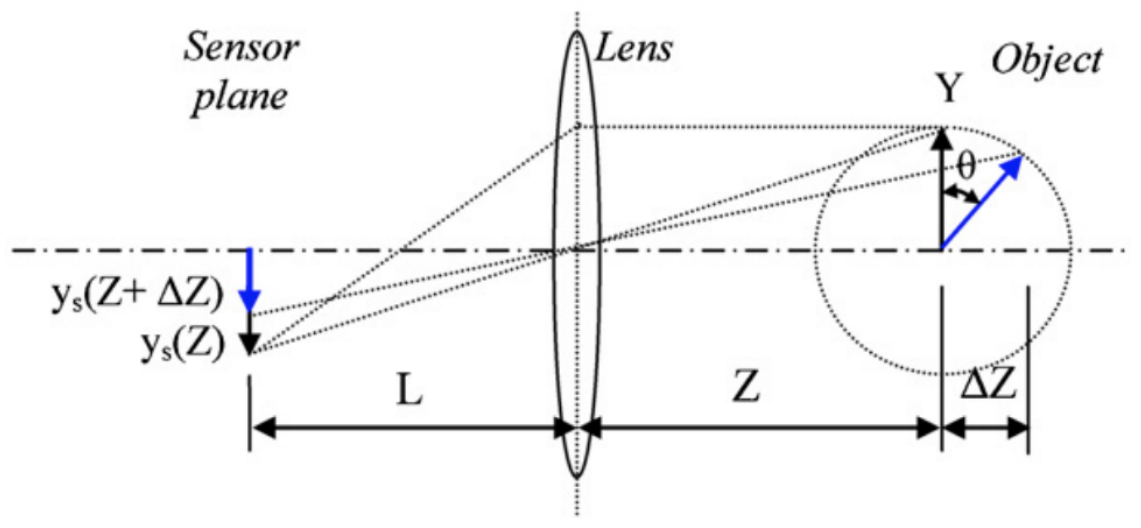

Figure 27: Effect of out-of-plane rotation on perceived feature location [15]. 
Partial derivatives of the displacement are used to derive the strains for out-ofplane rotation as the following:

$$
\begin{array}{r}
\varepsilon_{x x}=\frac{\partial U(\Delta Z)}{\partial x_{s}} \approx-\frac{Y \sin \theta}{Z} \\
\varepsilon_{y y}=\frac{\partial V(\Delta Z)}{\partial x_{s}} \approx \cos \theta-1-\frac{2 Y \sin \theta \cos \theta}{Z}=\cos \theta-1-\frac{Y \sin 2 \theta}{Z}
\end{array}
$$

Although literature suggests the only method of significantly reducing out-ofplane motion is to use a stereoscopic imaging system, this is not always feasible depending on the application. In such cases, artificial correction such as [48] may be implemented.

\subsection{X-ray Digital Image Correlation}

DIC algorithms can compute displacement and strain fields regardless of image acquisition method. In fact, the use of particle tracking and DIC using X-ray images it not a new concept. A variety of radiographic measurement techniques such as X-ray Diffraction, X-ray Phase Contrast Imaging (PCI), and computed tomography have all been used in the context of XDIC, this review will focus primarily on absorption radiography from tube generators (flash and rotating anode) and synchrotrons [34], [49], [50].

\subsubsection{X-ray Imaging Methods}

Flash X-ray is limited to tens of nanosecond exposure times, therefore one generator is insufficient for imaging continuous deformation events. Nonetheless, as early as [51], repeated flash X-ray were used to image unloaded and deformed fibre-reinforced composites quasi-statically. 
High speed dynamic XDIC studies (e.g., ballistics) are mostly performed using several flash X-ray systems capturing an event from several different angles. Image registration for multi-angle frames require high-contrast fiducial markers that provide reference features between the different views for proper alignment [24]. Imaging of dynamic events using a rotating anode generator eliminates the need for fiducial markers as continuous exposures are possible. Although with cineradiography, nanosecond exposure times of flash X-ray are not possible.

\subsubsection{X-ray Contrast}

Multiple methods of generating X-ray contrast were used in literature based on the experimental requirements (e.g., penetration depth, materials). Not all methods presented here are for digital image correlation; however, the methods could be implemented for displacement measurement or volume correlation. Optical DIC typically requires high contrast black and white speckles for proper measurement. This surface pattern is often achieved using spray paint to produce small black speckles on a white background coat. In the context of XDIC, contrast methods included surface applications and natural or artificial internal features.

The closest analogy for painted speckles in XDIC was the use of a rubberized paint containing steel spheres 37,74 , and $98 \mu \mathrm{m}$ in diameter applied on a glassepoxy laminate [51]. The authors also suggest mixing the steel spheres with a clear lacquer for better adhesion. To create surface contrast, it was also demonstrated that etching of aluminum provided sufficient detail for X-ray phase contrast imaging (Fig. 28) [34] .

Although surface methods generate sufficient X-ray contrast for DIC, applying lacquer to or etching the surface of a specimen does not allow for characterization of optically inaccessible internal displacement and strain fields. In these situations, 

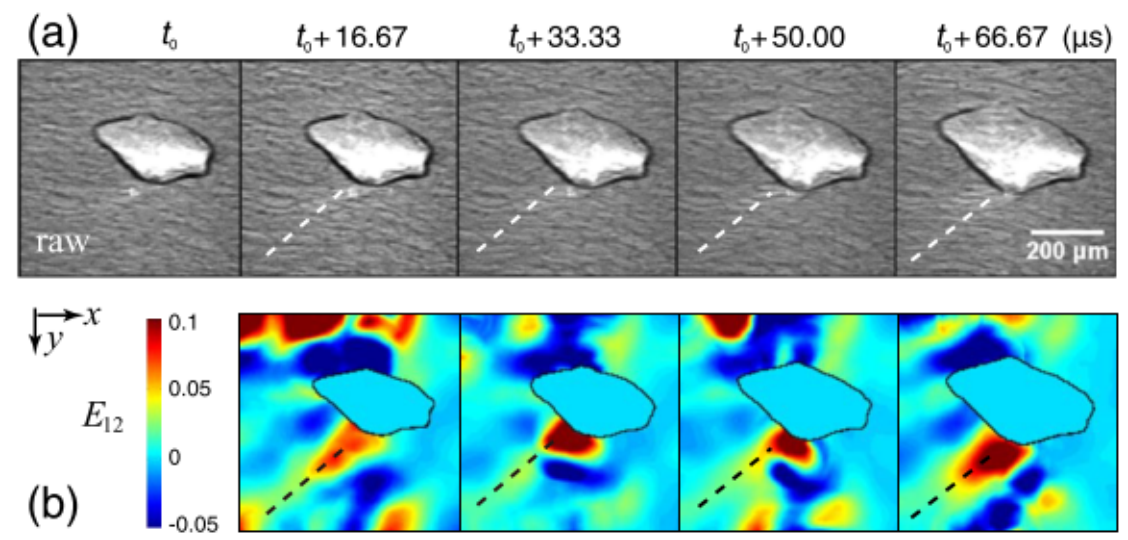

Figure 28: X-ray phase contrast image of aluminum with surface etching for crack propagation and DIC plots [34].

it may be beneficial to simply use optical techniques rather than radiography.

In certain applications, it is possible to take advantage of structural inhomogeneity to create contrast. This is of particular use for 3D CT studying of natural structures. The large difference between attenuation profiles of metals and air allow for naturally-occuring contrast in metal foams [52], [53]. Aluminum foam, shown under compression in CT reconstructions shown in Fig. 29.

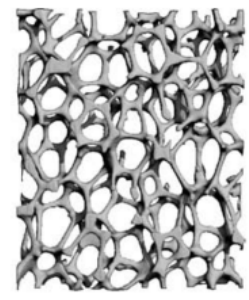

(0)

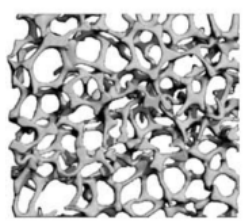

(4)

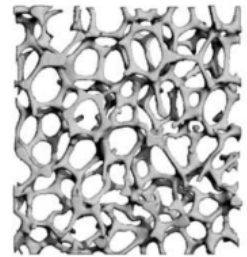

(1)

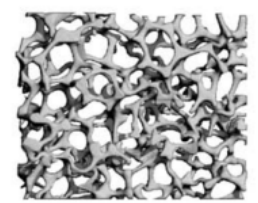

(5)

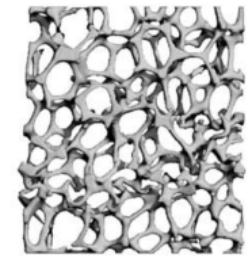

(2)

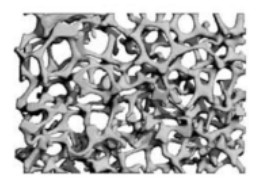

(6)

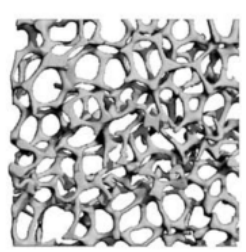

(3)

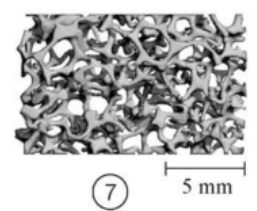

Figure 29: CT reconstruction of aluminum foam [53]. 
In specific applications, where the specimen is naturally composed of different materials, additional contrast may not be required. CT reconstruction of crack propagation in tropical wood was performed in [54]. Variations in attenuation coefficients for fibers within the wood provided sufficient contrast for reconstruction. X-ray microtomography for Volumetric DIC of argillaceous rock was also performed during triaxial compression tests [43]. The clay matrix containing calcite and quartz used density difference of interstratified layers for contrast. A reconstructed slice of argillaceous rock from [43] is shown in Fig. 30.

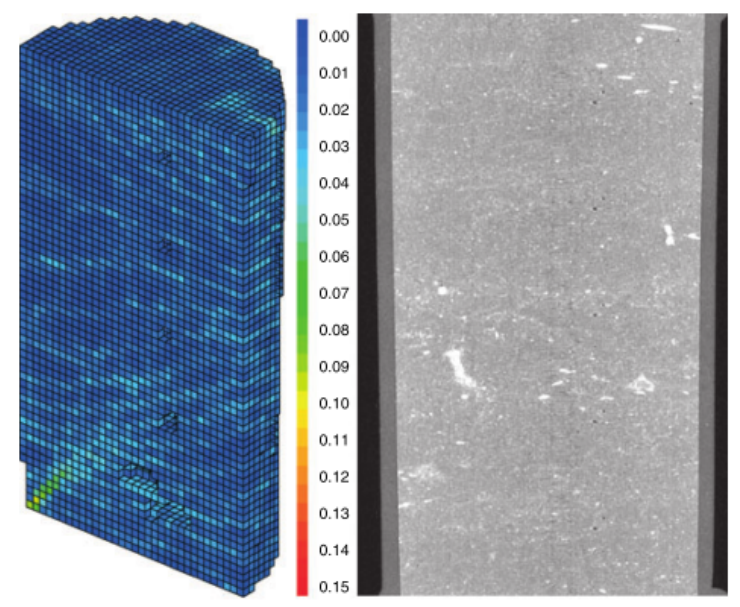

Figure 30: CT reconstruction of argillaceous rock using naturally occuring contrast and corresponding von-Mises strain [43].

Dynamic deformation of materials that cannot be quantified using optical methods or strain gauges is possible using XDIC. For example, granular materials such as sand do not have the continuity required for transducer integration and are not optically transparent. A method used by [55] was to sieve lead fillings in a central plane of sand prior to a gas-gun driven copper rod impact. Sand was also used for CT reconstruction under triaxial compression [56]. Wide-grained sand was shown to have sufficient spacing between particles that air was used as 


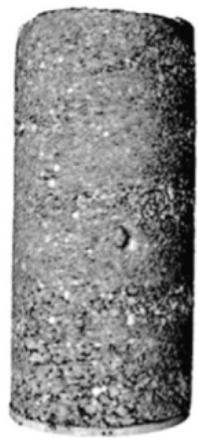

Initial

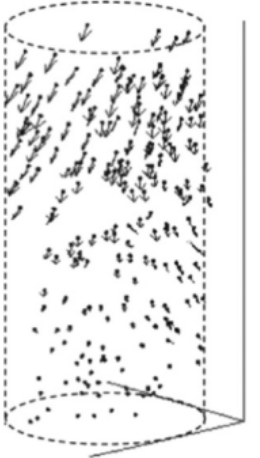

Initial-A

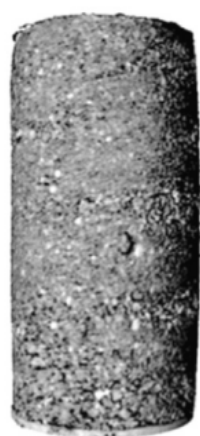

A

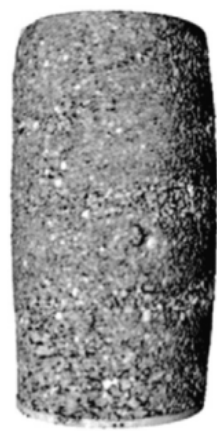

B

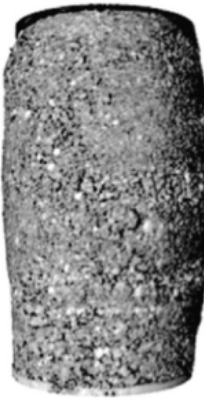

C

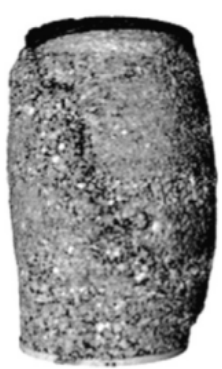

D

Figure 31: CT reconstruction of wide-grained sand in triaxial compression [56].

a contrasting medium.

In the case of ballistic and impact tests, the target itself can provide sufficient contrast relative to the projectile. For example, a steel rod impacting a borosilicate block [57] and projectile dwell analysis of a silicon carbide (SiC) ceramic plate (Fig. 32) [58] are possible using inherent contrast.

The final method of $\mathrm{X}$-ray contrast generation is embedding markers having a different composition within the bulk medium. A polyester specimen subjected penetration by steel spheres $(373.5 \pm 3.0 \mathrm{~m} / \mathrm{s})$ had lead shavings embeded in a plane parallel to the projectile, as shown in Fig. 33, during flash X-ray experiments [24]. Alternatively, borosilicate beads in poly(methyl methacrylate) (PMMA) provided sufficient contrast in X-ray phase contrast imaging for DIC analyses [34]. 


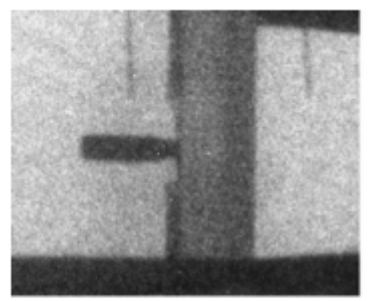

$5.9 \mu \mathrm{s}$

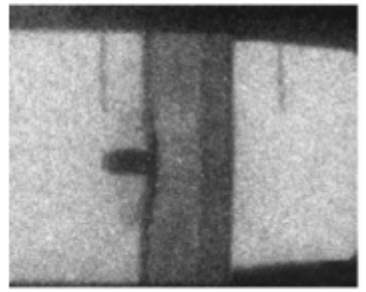

$25.3 \mu \mathrm{s}$

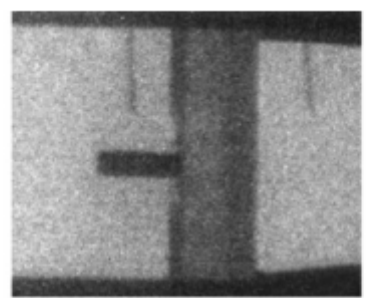

$10.8 \mu \mathrm{s}$

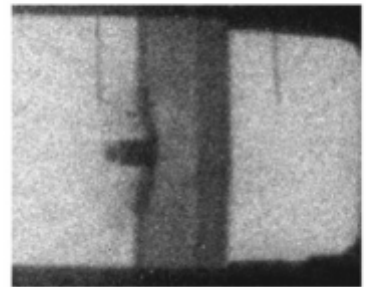

$30.4 \mu \mathrm{s}$

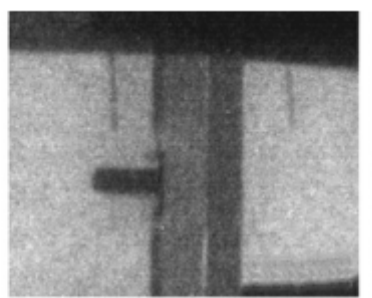

$15.7 \mu \mathrm{s}$

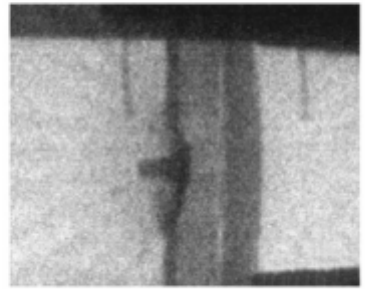

$35.5 \mu \mathrm{s}$

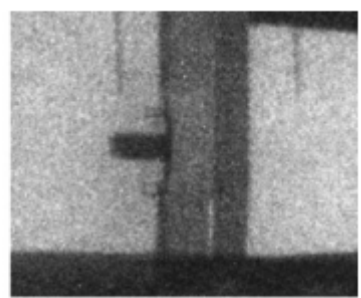

$20.7 \mu \mathrm{s}$

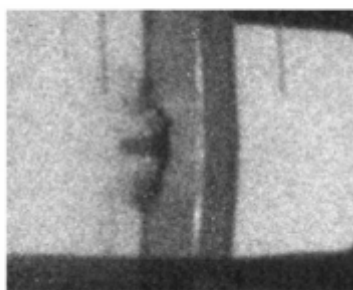

$40.6 \mu \mathrm{s}$

Figure 32: X-ray analysis of ballistic dwell in $\mathrm{SiC}$ penetration. [58].

Tungsten beads were used in [50] as contrast markers in an aluminum cylinder for synchrotron X-ray tomography. Embedding markers is performed during specimen creation in both of these examples, forming an essential component of the casting process.

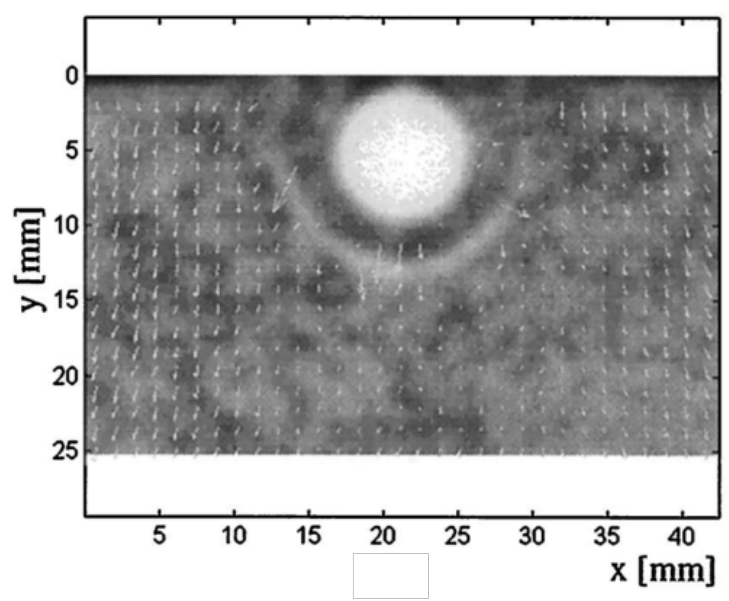

Figure 33: Flash X-ray of steel sphere penetrating polyester with embedded lead shavings [24].

In certain applications, due to practical or physical limitations, the markers 
cannot be cast into a specimen and rather must be inserted. This is the case for biological tissues. In both [59] and [60], radio-opaque markers were embedded in PMHS studies. Radiopaque ink-infused polystyrene foam $\left(\rho \approx 2.0-2.7 \mathrm{~g} / \mathrm{cm}^{3}\right)$ [59] and tin-filled polystyrene tubes $\left(\rho \approx 1.5 \mathrm{~g} / \mathrm{cm}^{3}\right)$ [60] were embedded in brain tissue $\left(\rho \approx 1.0 \mathrm{~g} / \mathrm{cm}^{3}\right)$, the latter is shown in Fig. 34. These markers required surgical insertion before X-ray imaging to characterize brain deformation during the impact.

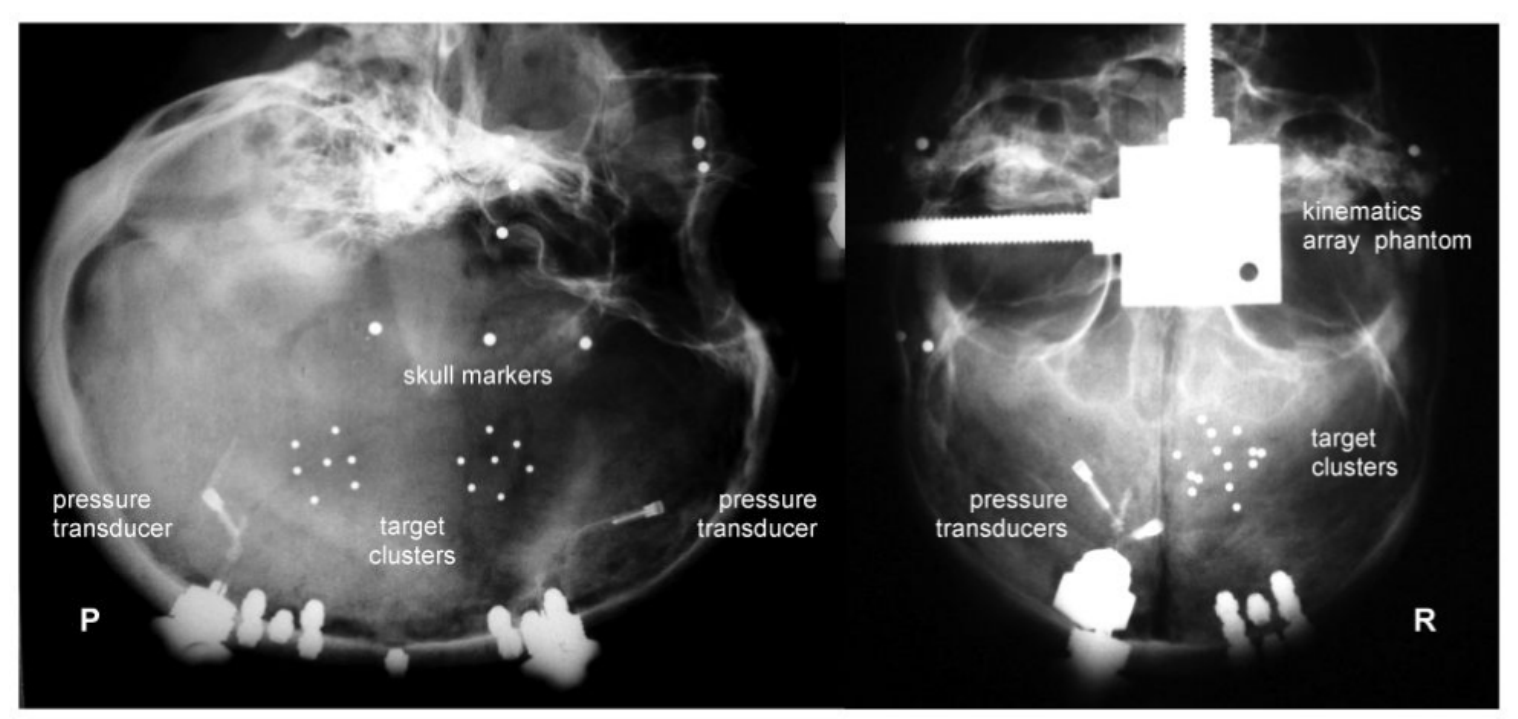

Figure 34: X-ray image of embedded tin/polystyrene contrast markers in a PMHS. [60].

Discussion regarding the importance of marker/specimen dynamic coupling in [60] notes the importance of neutral density markers. As the density difference between the markers and bulk material increases, there will be a corresponding difference in acceleration $(\mathrm{F}=\mathrm{ma})$. Therefore, the marker response may lag that of the bulk medium after large accelerations such as those seen in impact events. As XDIC tracks the projection of the markers and not the bulk material, the results can be unintentionally biased.

The inclusion of neutral density markers in a bulk specimen will increase dynamic coupling; however, further consideration is required for optimal marker 
design. Here, optimal is defined as providing sufficient contrast for accurate measurement while being otherwise minimally obtrusive to the bulk material properties. As this work focuses on the use of tissue simulants, soft polymers are used in all analyses, therefore marker design must consider material stiffness as an additional factor. If marker stiffness is not considered, the relative deformation of the markers will not match that of the bulk specimen. In the case where particles are significantly harder than the bulk medium, Particle Image Velocimetry (PIV) is more suited. PIV measures the displacement of each speckle individually, not providing true full field results.

X-ray contrast must be present for XDIC analyses. As discussed previously, certain applications use naturally occurring variations in specimen structure or material composition. However, in the majority of experiments, artificial contrast markers are required. Lead and tin are popular for their high attenuation coefficients; however, as discussed by [60], dynamic coupling must be considered. The next chapter proposes a method of predicting image quality based on contrast composition with the intent of designing minimally invasive markers. 


\section{Chapter 4}

\section{Analytical Models}

Analytical models were developed to increase the understanding and limitations of the high-speed X-ray facility. The models were developed to quantify measurement uncertainty caused by out-of-plane motion and to provide a method of contrast marker design.

\subsection{Modelling of Marker Contrast}

A critical design parameter for future high-speed X-ray studies of headforms and PMHS is the correct implementation of radiographic contrast markers inside the plane of interest. As discussed previously, there is an optimal marker composition for a given experiment such that the markers minimally influence the behaviour of the brain surrogate material, while providing sufficient contrast for accurate image correlation measurements. Although determining an optimal composition is beyond the scope of this work, it should be noted that increasing doping concentration will change both density and stiffness of the bulk material, while providing higher visibility (i.e., contrast). Thus, a tool to predict contrast-to-noise ratio (CNR) for a prescribed specimen was developed. This section will discuss implementation, use and limitations of the CNR tool developed in MatLab as a Graphical User 
Interface (GUI).

\subsubsection{Material Attenuation}

To quantify predicted CNR, it is first necessary to define materials used to create the specimen and contrast markers. The density and total mass attenuation coefficient for $1-150 \mathrm{keV}$ is required to properly represent the $\mathrm{X}$-ray tube present in our facility.

The XCOM database provides attenuation coefficients (Rayleigh, Compton, photoelectric effect, and pair production) for the first 100 elements at energy levels of $1 \mathrm{keV}$ to $100 \mathrm{GeV}$ [20]. Attenuation profiles for elements, compounds (e.g., $\mathrm{BaSO}_{4}$ ), or mixtures (e.g., 50\%wt $\mathrm{BaSO}_{4}-50 \%$ wt PDMS) can be queried. To simplify compilation of new materials, a Matlab GUI, shown in Fig. 35 was developed.

Although the coherent, incoherent and photoelectric effect attenuations are shown in the Fig. 35 GUI plots, only the total attenuation coefficients are used in computation. The GUI uses elemental data to construct the attenuation profiles of mixtures using the same method as the XCOM database. The attenuation can be approximated using the mass-fraction, $\frac{W i}{W}$, weighted-sum of its components [20] .

$$
\mu / \rho=\sum_{i} \frac{W i}{W}(\mu / \rho)_{i}
$$

The mixture density is required to transform mass attenuation coefficients to linear attenuation coefficients. The density can be specified by the user to represent either a theoretical or measured value. If the density is not specified, the rule of mixtures which assumes no solubility between the components is automatically 

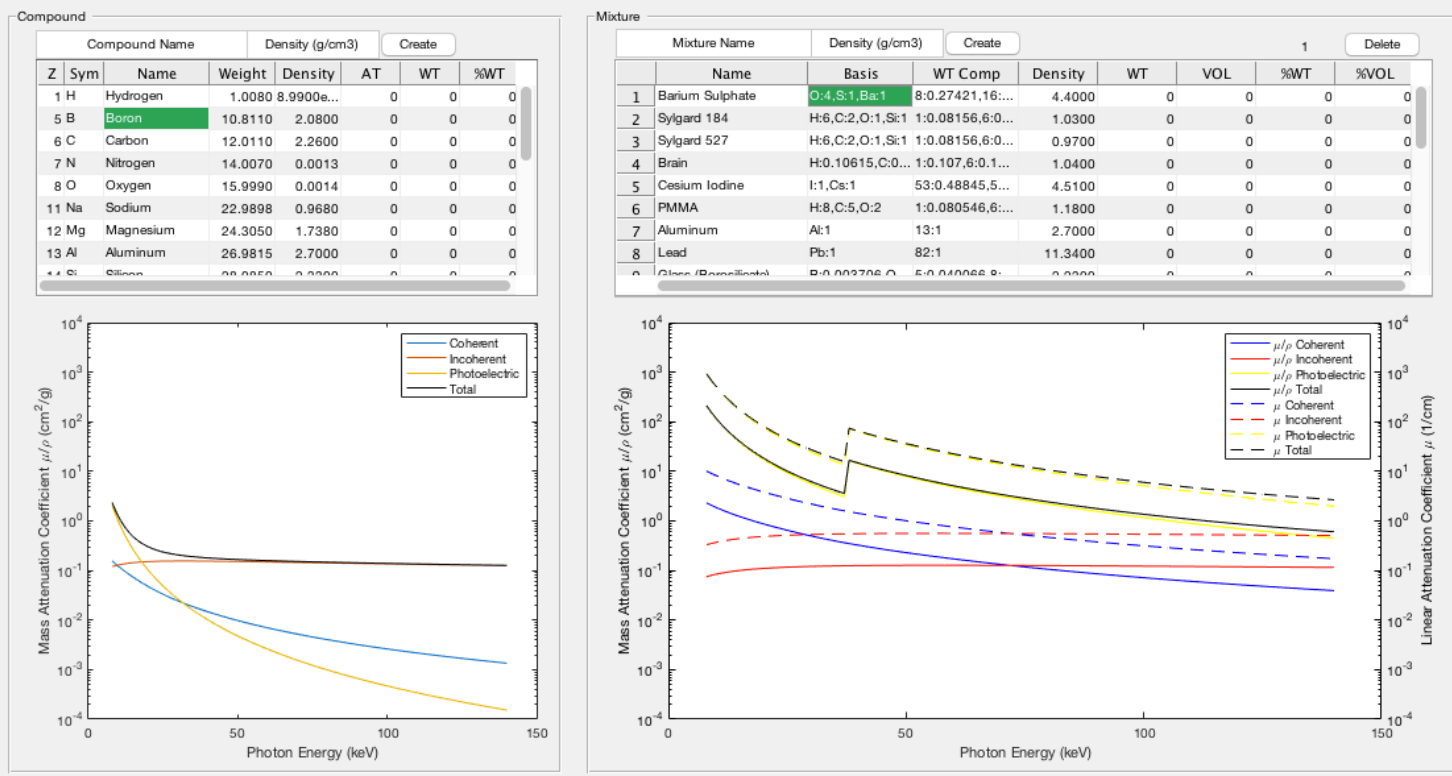

Figure 35: Matlab GUI to compute custom material attenuation coefficients. 
used, where the sum is weighted by volume fraction, $\frac{V_{i}}{V}$ [61].

$$
\rho=\sum_{i} \frac{V_{i}}{V}(\rho)_{i}
$$

\subsubsection{Layout Dimensions}

As several of the parameters, notably air KERMA and camera field of view depend on layout dimensions, CNR prediction inputs include five key dimensions. These dimensions define the locations of the an X-ray source, impact plane, scintillator, mirror, and camera. The purpose of the mirror is to protect the camera from direct exposure to the primary X-ray beam. The distance from the source to 1 " lead bricks designed to attenuate the main beam is also included to ensure the beam does not exceed safe bounds. Fig. 36 shows the layout and important dimensions that are used in the CNR computation tool GUI. Description of dimensions A-E are provided in 1

Table 1: Layout dimension definitions
A $\mid$ X-ray housing face to scintillator, source is inset by $76.2 \mathrm{~mm}$ (3.0 in).
B Impact plane to scintillator
Scintillator to mirror along centre axis
$\mathrm{D}$ Scintillator to lead wall (saftey verification)
E Centre axis to front of camera lens (lens mount panel)

\subsubsection{X-ray Spectrum}

The CNR prediction tool was designed for the equipment currently used in our lab at Carleton University, this includes our $150 \mathrm{kV}_{p}, 80 \mathrm{~kW}, 12^{\circ}$ Tungsten-Rhenium 


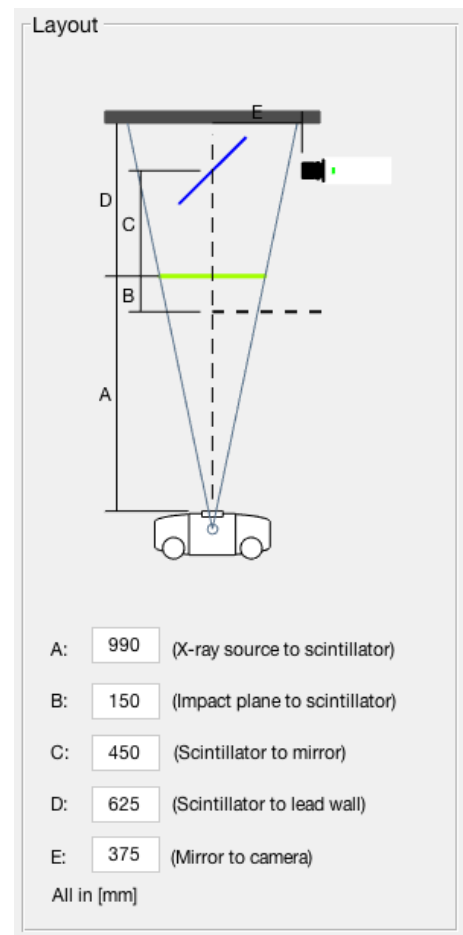

Figure 36: Matlab GUI to specify experiment dimensions

rotating anode $\mathrm{X}$-ray generator. For purposes of the computation tool, the tungsten anode specteral model using interpolatin polynomials (TASMIP), was implemented to produce a realistic spectrum [14]. Siemen's Simulation of X-ray Spectra (Online tool for the simulation of X-ray Spectra) [13] can also be used as it is an as the spectral output is based on work in [14] and [62], and can produce outputs ranging from $30-140 \mathrm{kV}_{p}$ for a Tungsten anode. Although the composition varies slightly and the output from [13] does not reach the $150 \mathrm{kV}_{p}$ of our system, it provides a sufficient estimation for energy levels up to $140 \mathrm{kV}_{p}$. The difference in composition will not significantly influence the spectral output, with only a slight shift in characteristic peaks expected.

By default, the spectral output of [13] has a Kinetic EneRgy per MAss of air 
(air KERMA) of $1 \mathrm{mGy}$. KERMA is an exposure dose, or a scaling factor for the $\mathrm{X}$ ray spectum to represent exposure energy that accounts for several factors including distance from source to detector, exposure time, pixel area, current and peak power. The TASMIP model includes an interpolation function for determining exposure at $1 \mathrm{~m}$ from the source in a tube similar to the one used in our facility [14]. Therefore, spectra from either [14] or [13] is corrected for distance and other factors to approximate the spectral fluence $(\Phi(E))$.

The KERMA can be corrected as follows to account for exposure and layoutspecific quantities [63]. KERMA is proportional to a power of the input voltage to the charge, and to the inverse distance between source and scintillator. The photon fluence per pixel, is a function of TASMIP spectrum at an input voltage $(\mathrm{kV})$ with the following factors:

$$
\Phi_{0}(E)=\Phi_{k V}(E) \frac{i_{g e n} A_{p s}}{Z^{2} f}
$$

where $i_{\text {gen }}$ is the generator current in $\mathrm{mA}, \mathrm{Z}$ is the distance from source to scintillator in metres, $r$ is the frame rate, and $A_{p s}$ is the equivalent area of one pixel projected on the scintillator. The CNR prediciton tool requires two inputs from the user in order to compute the incident X-ray spectrum, tube current and voltage as shown in Fig. 37. The tube voltage in $\mathrm{kV}$ will approximately correspond with a maximum X-ray photon energy in $\mathrm{keV}$.

\subsubsection{Camera Parameters}

A number of factors influence the quantity of light reaching the high-speed camera from the scintillator, including: the lens, camera software, and camera hardware. The GUI for user specified camera parameters is displayed in Fig.38. 


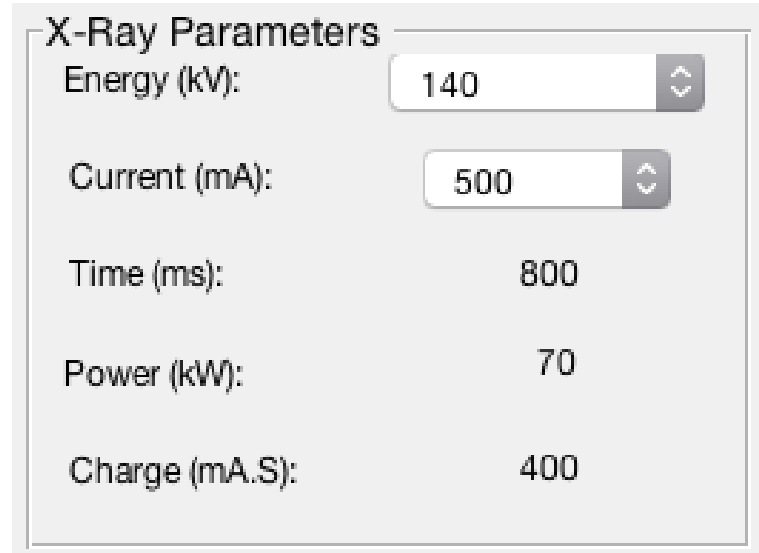

Figure 37: Matlab GUI to specify X-ray parameters

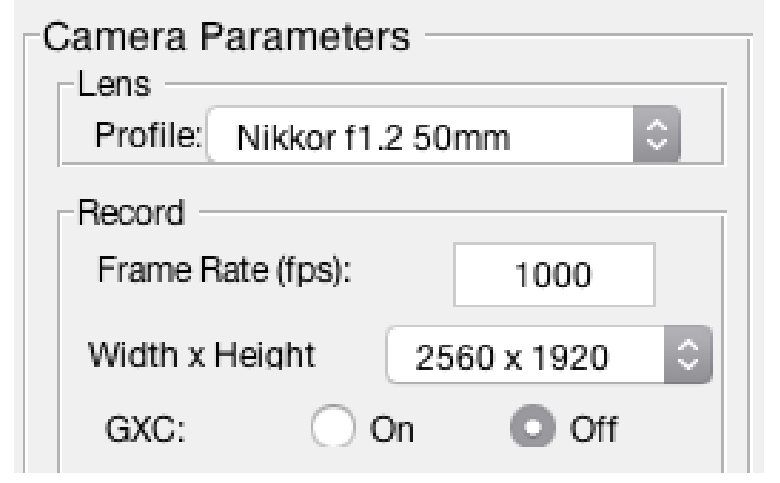

Figure 38: Matlab GUI to specify camera parameters 


\section{Camera Software Parameters}

Software parameters are considered to be recording settings set in camera software. Table 2 describes theses parameters and their use.

Table 2: Camera software parameters

\begin{tabular}{c|c} 
Record rate & Computed exposure time $=1 /$ (record rate $)$ \\
Resolution & Pixel count per frame \\
$2 \times 2$ Pixel binning & Increases brightness, reduces spatial resolution.
\end{tabular}

The resolution and frame rate are limited by the capabilities of the camera. As the frame rate increases, total resolution decreases. This limitation is a result of limited operation frequency on the chip (ie. total operations per second). For example, although the full resolution of $2560 \times 1820$ is possible up to $2000 \mathrm{fps}$, a maximum resolution of $1280 \times 720$ is possible at $10,000 \mathrm{fps}$.

\section{Camera Hardware Parameters}

Hardware parameters reflect built-in properties of the high-speed camera. The properties used are described in Table 3.

Table 3: Camera hardware parameters

\begin{tabular}{c|c} 
Sensor size & Sensor size for all pixels \\
Fill factor & Fraction of sensor area used to capture photons \\
Quantum efficiency & Spectral photon to electron conversion efficiency \\
Gain & Internal software camera gain
\end{tabular}

The properties descried in Table 3 are typically protected proprietary information and therefore their values have not been included in this description. It is 
critical when selecting a high-speed camera or scintillator to match the quantum efficiency curve to the scintillator spectral output curve for maximum light sensitivity. The fill factor is a measure of actual sensor area to total area per pixel.

\section{Camera Lens Parameters}

A significant factor influencing the number of photons reaching the sensor is the lens selection. The focal length, aperture size, and transmission, are all important factors in determining the field of view and light output.

The field of view was determined using the camera position, position of sensor within the camera, focal length, and lens length using the following equation [64].

$$
F O V=h_{\text {sens }} D_{w} / L
$$

Where the FOV represents the field of view, $h_{\text {sens }}$, the sensor size, $L$, the focal length and $D_{w}$, the working distance. The working distance includes the distance from scintillator to mirror and mirror to lens centre. Note the FOV will be different in each direction to reflect the aspect ratio.

The lens transmission is another consideration. A large apeture (low f-number) is beneficial as more photons reach the sensor. In this application, the camera is imaging a flat plane (scintillator), therefore depth of field is not important, a fast lens should be used. The lens dimensions and transmission are required. The lens transmission is measured in T-stops, a value not often provided by manufacturers. Online databases such as DXOmark have experimentally determined T-stop values for a range of lenses, defined as the equivalent F-stop that would result in $100 \%$ transmission [65]. The database values can be used to compute loss factors. For example, an $\mathrm{f} / 1.4$ lens that is evaluated to have a $\mathrm{t} / 2.0$ rating, has a loss factor of 1 full stop $(2.0 / 1.4 \approx \sqrt{2})$ or a $50 \%$ reduction in light transmission. 


\subsubsection{Specimen Layers}

Attenuation profiles are computed for a specimen constructed in layers. Filters occuring before the specimen (e.g., $\mathrm{X}$-ray housing window), and after the specimen (e.g., scintillator mount and anti-scatter grids) can be specified as pre-/post-filters. The specimen is defined by layers containing or absent of contrast markers.

Photon attenuation is computed along three paths: through only filters, through the bulk specimen without markers, and through the specimen along a path containing contrast markers. Table 4 indicates which layers are included in the definition of each path.

Table 4: Attenuation path definitions

\begin{tabular}{|c|c|c|c|c|} 
& Pre-filters & Bulk medium & Contrast markers & Post-filters \\
Filter Path & $\mathrm{x}$ & - & - & $\mathrm{x}$ \\
Specimen Path & $\mathrm{x}$ & $\mathrm{x}$ & - & $\mathrm{x}$ \\
Marker Path & $\mathrm{x}$ & $*$ & $\mathrm{x}$ & $\mathrm{x}$
\end{tabular}

The only distinction between attenuation profiles computed along a specimen path and a marker path is compositions of the marker layer. For example, a headform with a $150 \mathrm{~mm}$-thick PDMS brain containing a $2 \mathrm{~mm}$ slice of $50 \mathrm{wt} \% \mathrm{BaSO}_{4}{ }^{-}$ $50 \mathrm{wt} \%$ PDMS would have a specimen cross section of $150 \mathrm{~mm}$ PDMS, while the marker path has $148 \mathrm{~mm}$ PDMS and $2 \mathrm{~mm}$ of $50 \mathrm{wt} \% \mathrm{BaSO}_{4}-50 \mathrm{wt} \%$ PDMS.

The user specifies pre- and post-specimen filters by defining the material, thickness and location relative to the specimen. The cross-sections are similarly defined by material, dimensions, and marker composition, if present, as shown in Fig. 39. Although the only dimensions used in CNR computation are the penetration depth and marker diameter, the specimen width and marker spacing is also included for 


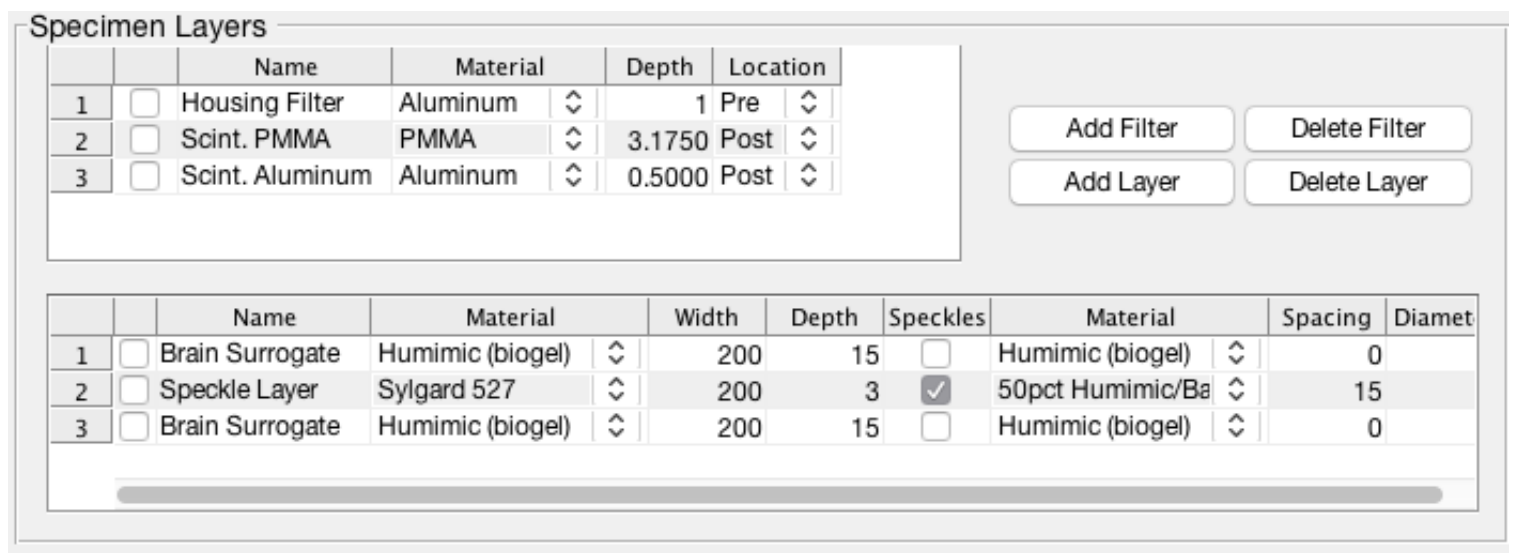

Figure 39: Matlab GUI to specify specimen cross section composition

visualization purposes.

\subsubsection{Scintillator Properties}

The scintillator information required for $\mathrm{CNR}$ computation are the thickness, mass attenuation coefficients, mass energy absorption coefficients, and photon conversion efficiency. The outer scintillator dimensions are also used with the field of view (lens parameters) and specimen dimensions to display a preliminary represenation of the expected image. The previewed render and example dimension calculations are shown in Fig. 40.

\subsubsection{Limitations/Assumptions}

The CNR computation tool described in this chapter is a preliminary tool to assess the validity of a potential marker design. A number of assumptions were required to develop the computation tool, limiting the accuracy of results. When possible, conservative approaches were used; however, the CNR should nonetheless be used as an upper bound on expected contrast marker performance. Literature 

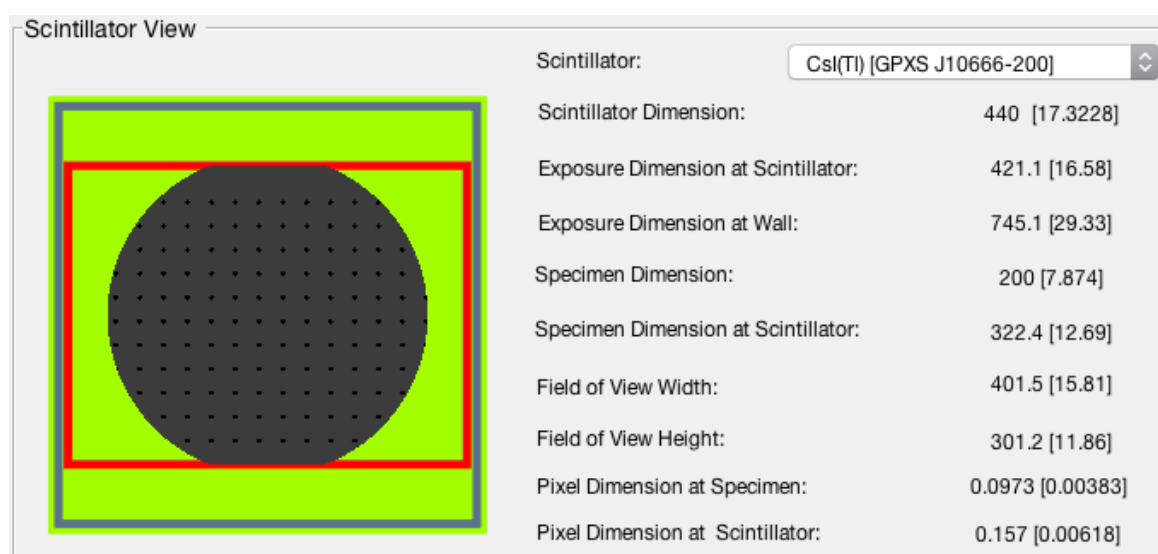

Figure 40: Matlab GUI for scintillator selection and to display computed viewrelated dimensions and representative cross-section. The green square is the scintillator, the grey and red boxes are the exposed field and camera field of view, respectively.

suggests the output of a CsI(Tl) scintillator is $\approx 56-66$ photons $/ \mathrm{keV}$ of absorbed energy [66], [23], [67].

Two geometric factors not included in the simulation that influence image uniformity are the heel effect and conical projection lengths. The heel effect is caused by attenuation within the focal spot, preferentially on the anode side of the image, as shown in Fig. 41, resulting in a reduction in image intensity as per Fig. 42 [68] .

The attenuation is calculated assuming the incident beam is normal to the sensor. In practice, the X-ray intensity will be highest in a straight line from the source, and the edges will be darker. The inverse square law and obliquity result in an image intensity that varies with the field of view according to the following [3]:

$$
I_{d}(x, y)=I_{0} \cos ^{3} x
$$

Eq. 66 requires the number of pixels spanned by a speckle to calculate the final CNR. A theoretical area is calculated using the marker dimension and pixel size projected onto the scintillator. As discussed in Sec. 2.1.4, the pixel span of each 


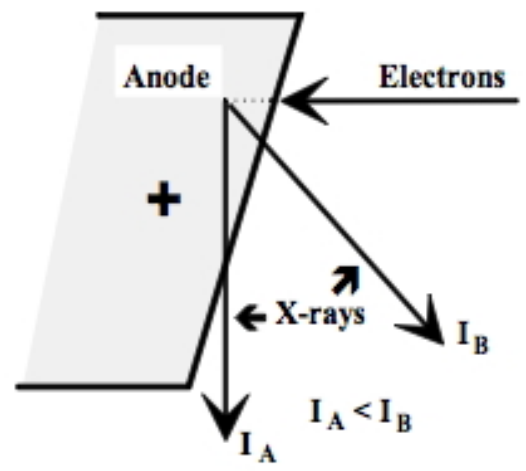

Figure 41: Cause of the heel effect, beams produced in the anode are attenuated, with emission higher attenuation as their angle approaches the anode surface angle caused by travelling a longer path [68].
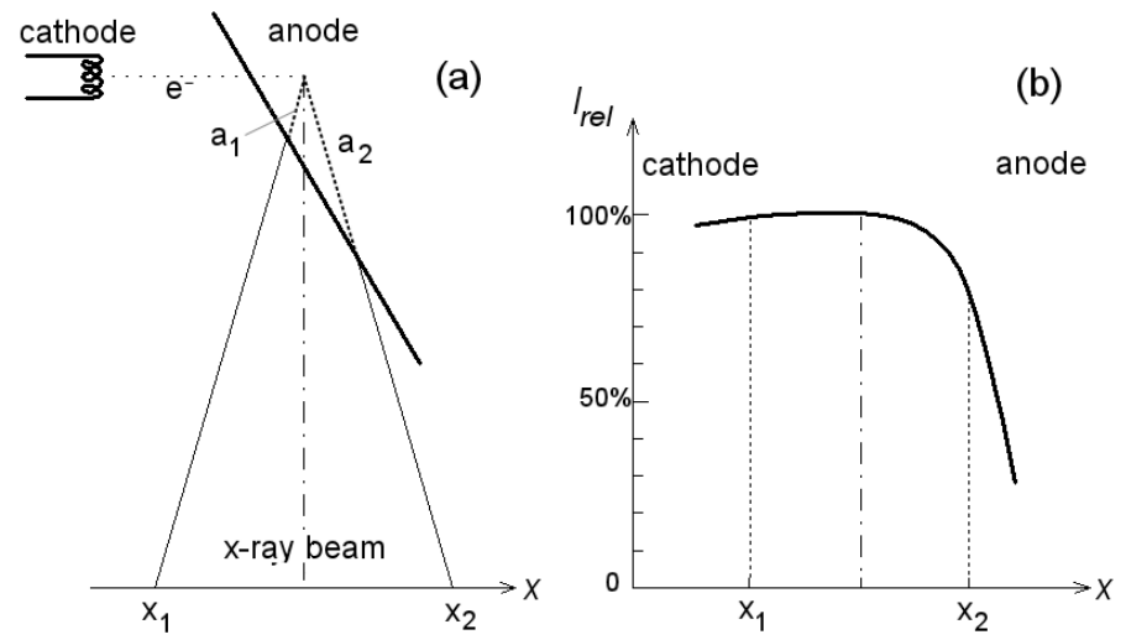

Figure 42: Resulting intensity profile along the sensor caused by the heel effect, OIAEA [69]. 
speckle and the edge sharpness is modified by edge blurring caused by finite focal spot size. The effect of edge sharpness and slight variations in perceived speckle size over the field of view were not quantified.

The contrast is reduced by scattered phtons reaching the sensor along a deviated trajectory after material interactions through either the Rayleigh or Compton processes. This reduction in image quality could be more accurately quantified by implementing Monte Carlo simulations, however, this is beyond the scope of a preliminary computation tool.

Despite several limitations caused by geometry, speckle size and scatter, the contrast-to-noise simulation tool is a valuable tool for preliminary marker design, providing an upper bound on expected contrast.

\subsection{Out-of-Plane Motion}

Out-of-plane motion is a known source of error in 2D DIC, with its impact described in Sec. 3.2.3. Current literature defines a 2D model without applied strain to quantify the expected error between experimental and computed strains [15] . This method has two limitations. First, a 2D model only allows for rotation about one axis, with the strain error computed as normal and parallel to the axis of rotation, and second, the discussion on applied strain with out-of-plane motion has not been quantified. Therefore, a 3D model allowing for out-of-plane translation and rotation about two perpendicular axes was developed to better quantify errors in realistic experimental situations. First, it is assumed that the strain state of an infinitesimal element can be approximated as a first-order model as shown in Fig. 43. 


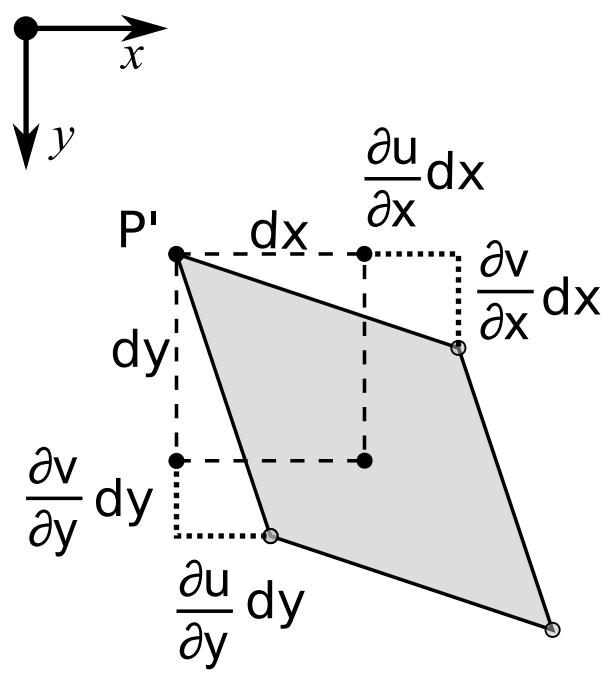

Figure 43: First order strain element

The general form of the out-of-plane motion including a strained element, biaxial rotation and translation is shown in Fig. 43. The notation used earlier in Ch. 2 and in Fig. 43 uses $P$ to describe the undeformed point, $P^{\prime}$ for the strained element, and now Fig. 44 introduces $P^{\prime \prime}$ as the coordinates after out-of-plane motion. Due to different $z$ coordinates of the points forming the strain element, the linear magnification onto the scintillator will not be constant. As such, the shape of the strain element and its projection are not identical, leading to the difference in applied versus perceived strain. In Fig. 44, the difference of shape for the object and its projection is clearly shown.

\subsubsection{Derivation}

This section will summarize the process used to derive perceived strain equations based on out-of-plane motion. This is a simplified version while the full derivation is included in Appendix B. The original location of strain element point $P$ and its 


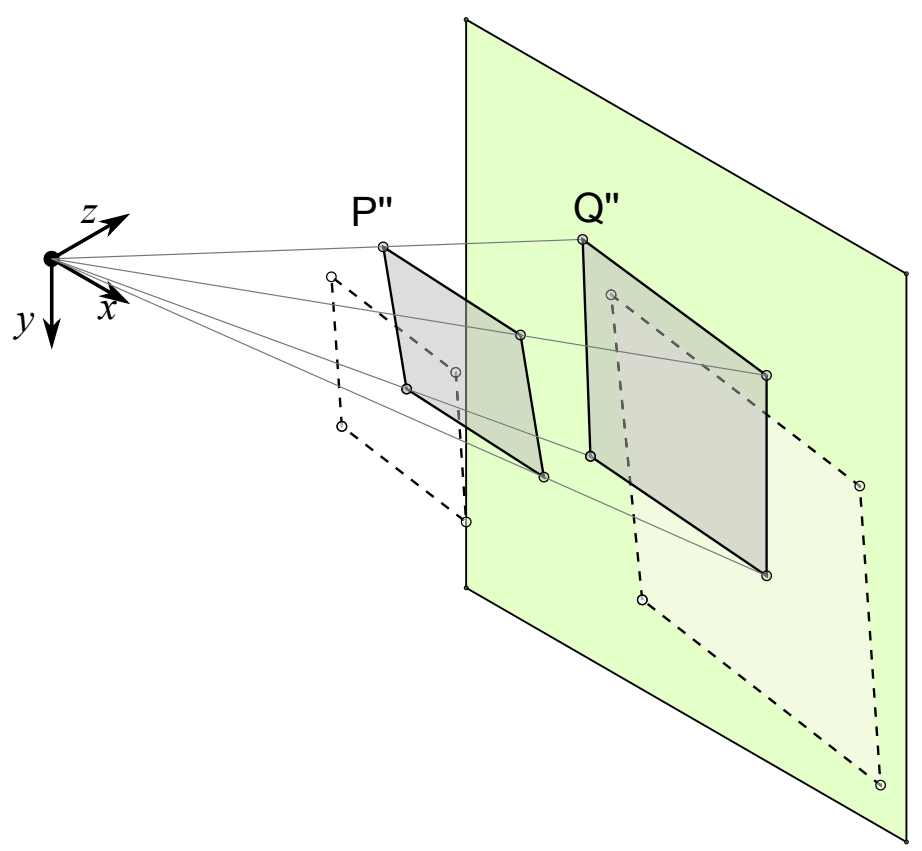

Figure 44: Projection of the strained element after out-of-plane translation and biaxial rotation.

location after in-plane strain is applied are:

$$
\begin{aligned}
P & =(x+u(x, y), y+v(x, y), z) \\
P^{\prime} & =(x+u(x, y), y+v(x, y), z)
\end{aligned}
$$

The applied out-of-plane motion is defined here as rotation about the $y^{\prime}$ axis, followed by rotation about the $x^{\prime}$ axis, followed by a translation along $z^{\prime}$. The $x^{\prime}, y^{\prime}$, and $z^{\prime}$ axes are located at an arbitrary point along the original object plane.

$$
O_{r}=\left(O_{r, x}, O_{r, x}, O_{r, z}\right)=\left(O_{r, x}, O_{r, x}, z\right)
$$

Therefore, the point coordinates after out-of-plane motion is:

$$
\left\{P_{i}^{\prime \prime}\right\}=\left[R_{x y z}\right]\left\{P_{i}^{\prime}-O_{r}\right\}+\left\{O_{r}+\Delta z\right\}
$$


where $\left[R_{z y x}\right]$ is the general 3D rotation matrix. For completeness, the equations were derived to include in-plane rotation about the $z^{\prime}$ axis. The matrix describing $3 \mathrm{D}$ rotation about $z^{\prime}$, then $y^{\prime}$, then $x^{\prime}$ is:

$$
\left[\begin{array}{ccc}
\cos \theta_{y} \cos \theta_{z} & -\cos \theta_{y} \sin \theta_{z} & \sin \theta_{y} \\
\cos \theta_{x} \sin \theta_{z}+\cos \theta_{z} \sin \theta_{x} \sin \theta_{y} & \cos \theta_{x} \cos \theta_{z}-\sin \theta_{x} \sin \theta_{y} \sin \theta_{z} & -\cos \theta_{y} \sin \theta_{x} \\
\sin \theta_{x} \sin \theta_{z}-\cos \theta_{x} \cos \theta_{z} \sin \theta_{y} & \cos \theta_{z} \sin \theta_{x}+\cos \theta_{x} \sin \theta_{y} \sin \theta_{z} & \cos \theta_{x} \cos \theta_{y}
\end{array}\right]
$$

Alternately expressed as:

$$
R_{z y x}=\left[\begin{array}{lll}
a_{11} & a_{12} & a_{13} \\
a_{21} & a_{22} & a_{23} \\
a_{31} & a_{32} & a_{33}
\end{array}\right]
$$

The transformed coordinates of point $P^{\prime}$ become:

$$
\begin{array}{r}
\left\{P^{\prime \prime}\right\}=\left(\left(x+u(x, y)-O_{x}\right) a_{11}+\left(y+v(x, y)-O_{y}\right) a_{12}+O_{x}\right. \\
\left(x+u(x, y)-O_{x}\right) a_{21}+\left(y+v(x, y)-O_{y}\right) a_{22}+O_{y} \\
\left.z+\Delta z+\left(x+u(x, y)-O_{x}\right) a_{31}+\left(y+v(x, y)-O_{y}\right) a_{32}\right)
\end{array}
$$


The linear projection of each coordinate on the scintillator is dependent on the position relative to the source. The general form of which is:

$$
Q_{i}^{\prime \prime}=\left(P_{i, x}^{\prime \prime} \frac{Z}{P_{i, z}^{\prime \prime}}, P_{i, y}^{\prime \prime} \frac{Z}{P_{i, z}^{\prime \prime}}, Z\right)
$$

These coordinates are slightly modified to reflect their use in DIC. To format displacements and strains, a scaling factor in [units]/[pixel] is required as an input parameter. This scaling factor is equivalent to $z / Z$. Therefore, the image coordinates, $R_{i}^{\prime \prime}$ are related to sensor coordinates $Q_{i}^{\prime \prime}$ by

$$
R_{i}^{\prime \prime}=\left(Q_{i, x}^{\prime \prime} \frac{z}{Z}, Q_{i, y}^{\prime \prime} \frac{z}{Z}\right)
$$

leading to

$$
\begin{aligned}
\left\{R^{\prime \prime}\right\}= & \left(\frac{z\left(\left(x+u(x, y)-O_{x}\right) a_{11}+\left(y+v(x, y)-O_{y}\right) a_{12}+O_{x}\right)}{z+\Delta z+\left(x+u(x, y)-O_{x}\right) a_{31}+\left(y+v(x, y)-O_{y}\right) a_{32}},\right. \\
& \left.\frac{z\left(\left(x+u(x, y)-O_{x}\right) a_{21}+\left(y+v(x, y)-O_{y}\right) a_{22}+O_{y}\right)}{z+\Delta z+\left(x+u(x, y)-O_{x}\right) a_{31}+\left(y+v(x, y)-O_{y}\right) a_{32}}\right)
\end{aligned}
$$

The perceived deformation between the original and transformed image points is:

$$
\begin{aligned}
& R_{x}^{\prime \prime}-R_{x}=u^{\prime}(x, y) \\
& R_{y}^{\prime \prime}-R_{y}=v^{\prime}(x, y)
\end{aligned}
$$

with the associated perceived strains expressed as 


$$
\begin{array}{r}
\varepsilon_{x x}^{\prime}=\frac{\partial u^{\prime}}{\partial x} \\
\varepsilon_{y y}^{\prime}=\frac{\partial v^{\prime}}{\partial y} \\
\varepsilon_{x y}^{\prime}=\frac{\frac{\partial u^{\prime}}{\partial y}}{1+\frac{\partial v^{\prime}}{\partial y}} \\
\varepsilon_{y x}^{\prime}=\frac{\frac{\partial v^{\prime}}{\partial x}}{1+\frac{\partial u^{\prime}}{\partial x}}
\end{array}
$$

The displacements expand to:

$$
\begin{aligned}
& u^{\prime}(x, y)=\frac{z\left(\left(x+u(x, y)-O_{x}\right) a_{11}+\left(y+v(x, y)-O_{y}\right) a_{12}+O_{x}\right)}{z+\Delta z+\left(x+u(x, y)-O_{x}\right) a_{31}+\left(y+v(x, y)-O_{y}\right) a_{32}}-x \\
& v^{\prime}(x, y)=\frac{z\left(\left(x+u(x, y)-O_{x}\right) a_{21}+\left(y+v(x, y)-O_{y}\right) a_{22}+O_{y}\right)}{z+\Delta z+\left(x+u(x, y)-O_{x}\right) a_{31}+\left(y+v(x, y)-O_{y}\right) a_{32}}-y
\end{aligned}
$$

To maintain compatibility with typical DIC algorithms, the deformations $u(x, y)$ and $v(x, y)$ are limited to the following first-order definitions:

$$
\begin{aligned}
& u(x, y)=u+\frac{\partial u}{\partial x} d x+\frac{\partial u}{\partial y} d y \\
& y(x, y)=v+\frac{\partial v}{\partial x} d x+\frac{\partial v}{\partial y} d y
\end{aligned}
$$

The derivation of the following strain equations is shown in Appendix B, with the 
following results for general perceived strain equations:

$$
\begin{aligned}
& \varepsilon_{x x}^{\prime}= \\
& \left(1+\frac{\partial u(x, y)}{\partial x}\right)\left((z+\Delta z) a_{11}+\left(y+v(x, y)-O_{y}\right)\left(a_{11} a_{32}-a_{12} a_{31}\right)-a_{31} O_{x}\right) \\
& =z \frac{+\left(\frac{\partial v(x, y)}{\partial x}\right)\left((z+\Delta z) a_{12}+\left(x+u(x, y)-O_{x}\right)\left(a_{12} a_{31}-a_{11} a_{32}\right)-a_{32} O_{x}\right)}{\left(z+\Delta z+\left(x+u(x, y)-O_{x}\right) a_{31}+\left(y+v(x, y)-O_{y}\right) a_{32}\right)^{2}}-1 \\
& \varepsilon_{y y}^{\prime}= \\
& \left(\frac{\partial u(x, y)}{\partial y}\right)\left((z+\Delta z) a_{21}+\left(y+v(x, y)-O_{y}\right)\left(a_{21} a_{32}-a_{22} a_{31}\right)+a_{31} O_{y}\right) \\
& =z \frac{+\left(1+\frac{\partial v(x, y)}{\partial y}\right)\left((z+\Delta z) a_{22}+\left(x+u(x, y)-O_{x}\right)\left(a_{22} a_{31}-a_{21} a_{32}\right)+a_{32} O_{y}\right)}{\left(z+\Delta z+\left(x+u(x, y)-O_{x}\right) a_{31}+\left(y+v(x, y)-O_{y}\right) a_{32}\right)^{2}}-1 \\
& \varepsilon_{x y}^{\prime}= \\
& \left(\frac{\partial u(x, y)}{\partial y}\right)\left((z+\Delta z) a_{11}+\left(y+v(x, y)-O_{y}\right)\left(a_{11} a_{32}-a_{12} a_{31}\right)-a_{31} O_{x}\right) \\
& +\left(1+\frac{\partial v(x, y)}{\partial y}\right)\left((z+\Delta z) a_{12}+\left(x+u(x, y)-O_{x}\right)\left(a_{12} a_{31}-a_{11} a_{32}\right)-a_{32} O_{x}\right) \\
& \left(\frac{\partial u(x, y)}{\partial y}\right)\left((z+\Delta z) a_{21}+\left(y+v(x, y)-O_{y}\right)\left(a_{21} a_{32}-a_{22} a_{31}\right)+a_{31} O_{y}\right) \\
& +\left(1+\frac{\partial v(x, y)}{\partial y}\right)\left((z+\Delta z) a_{22}+\left(x+u(x, y)-O_{x}\right)\left(a_{22} a_{31}-a_{21} a_{32}\right)+a_{32} O_{y}\right)
\end{aligned}
$$




$$
\begin{aligned}
\varepsilon_{y x}^{\prime}= & \\
& \begin{aligned}
(1+ & \left.\frac{\partial u(x, y)}{\partial x}\right)\left((z+\Delta z) a_{21}+\left(y+v(x, y)-O_{y}\right)\left(a_{21} a_{32}-a_{22} a_{31}\right)-a_{31} O_{y}\right) \\
& \quad+\left(\frac{\partial v(x, y)}{\partial x}\right)\left((z+\Delta z) a_{22}+\left(x+u(x, y)-O_{x}\right)\left(a_{22} a_{31}-a_{21} a_{32}\right)-a_{32} O_{y}\right) \\
& \frac{\left(1+\frac{\partial u(x, y)}{\partial x}\right)\left((z+\Delta z) a_{11}+\left(y+v(x, y)-O_{y}\right)\left(a_{11} a_{32}-a_{12} a_{31}\right)-a_{31} O_{x}\right)}{} \\
& +\left(\frac{\partial v(x, y)}{\partial x}\right)\left((z+\Delta z) a_{12}+\left(x+u(x, y)-O_{x}\right)\left(a_{12} a_{31}-a_{11} a_{32}\right)-a_{32} O_{x}\right)
\end{aligned}
\end{aligned}
$$

Although these equations may frighten even the most passionate out-of-plane enthusiasts (i.e., skydivers), they can be reduced to quantify anticipated uncertainties in specific applications. For example, for pure out-of-plane translation with no rotation (Fig. 45), or for uni-axial rotation without translation about the $y^{\prime}$ axis, the equations are simplified.

Pure translation along $z^{\prime}: \theta_{x}=0, \theta_{y}=0, \theta_{z}=0$

$$
\begin{gathered}
a_{11}=1, a_{12}=0, a_{21}=0, a_{22}=1, a_{31}=0, \text { and } a_{32}=0 \\
\varepsilon_{x x}^{\prime}=z \frac{\left(1+\varepsilon_{x x}\right)}{(z+\Delta z)}-1 \\
\varepsilon_{y y}^{\prime}=z \frac{\left(1+\varepsilon_{y y}\right)}{(z+\Delta z)}-1 \\
\varepsilon_{x y}^{\prime}=\frac{\left(\frac{\partial u(x, y)}{\partial y}\right)}{\left(1+\varepsilon_{y y}\right)}=\varepsilon_{x y}
\end{gathered}
$$




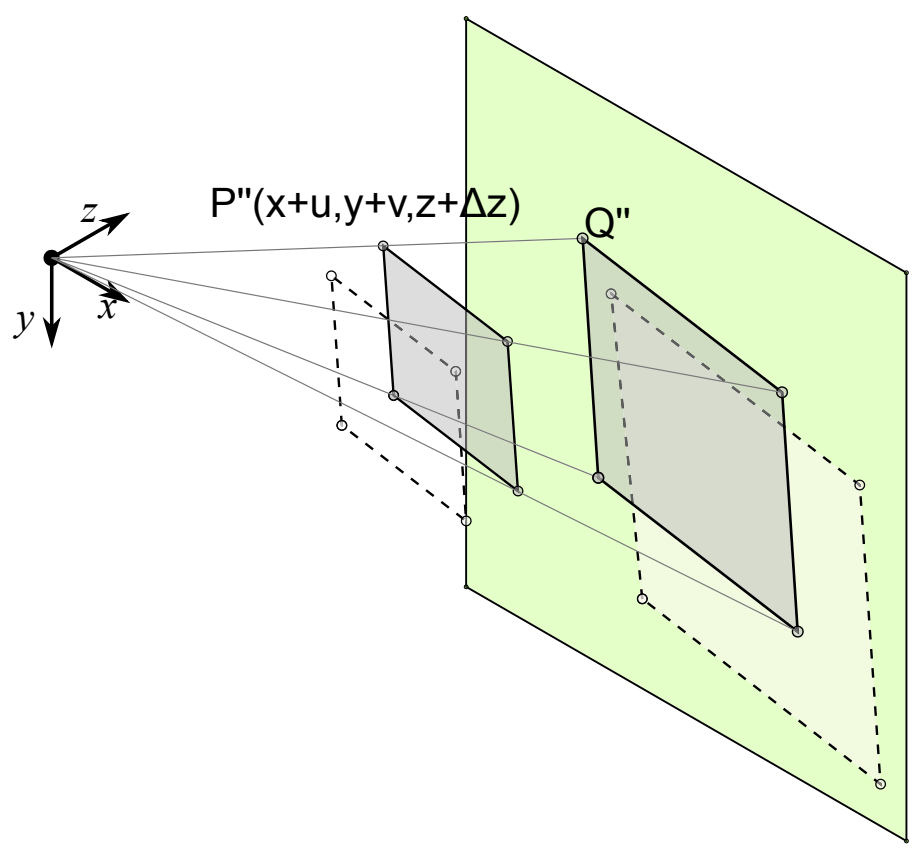

Figure 45: Projection of the strained element after out-of-plane translation.

$$
\varepsilon_{y x}^{\prime}=\frac{\left(\frac{\partial v(x, y)}{\partial x}\right)}{\left(1+\varepsilon_{x x}\right)}=\varepsilon_{y x}
$$

Pure rotation about $y: \theta_{x}=0, \theta_{z}=0, \Delta \mathbf{z}=\mathbf{0}$

$$
\begin{gathered}
a_{11}=\cos \theta_{y}, a_{12}=0, a_{21}=0, a_{22}=1, a_{31}=-\sin \theta_{y}, \text { and } a_{32}=0 . \\
\varepsilon_{x x}^{\prime}=z \frac{\left(1+\varepsilon_{x x}\right)\left(\left(z \cos \theta_{y}+\sin \theta_{y} O_{x}\right)\right.}{\left(z-\sin \theta_{y}\left(x+u(x, y)-O_{x}\right)\right)^{2}}-1
\end{gathered}
$$




$$
\begin{aligned}
& \left(\frac{\partial u(x, y)}{\partial y}\right)(y+v(x, y))\left(\sin \theta_{y}\right) \\
& \varepsilon_{y y}^{\prime}=z \frac{+\left(1+\varepsilon_{y y}\right)\left((z)-\left(x+u(x, y)-O_{x}\right)\left(\sin \theta_{y}\right)\right)}{\left(z-\left(x+u(x, y)-O_{x}\right)\left(\sin \theta_{y}\right)\right)^{2}}-1 \\
& \varepsilon_{x y}^{\prime}= \\
& \left(\frac{\partial u(x, y)}{\partial y}\right)\left((z) \cos \theta_{y}+\left(y+v(x, y)-O_{y}\right)\left(\sin \theta_{y} O_{x}\right)\right. \\
& \left(\frac{\partial u(x, y)}{\partial y}\right)\left(\left(y+v(x, y)-O_{y}\right)\left(\sin \theta_{y}\right)-\sin \theta_{y} O_{y}\right) \\
& +\left(1+\varepsilon_{y y}\right)\left((z)-\left(x+u(x, y)-O_{x}\right)\left(\sin \theta_{y}\right)\right) \\
& \varepsilon_{y x}^{\prime}= \\
& \left(1+\varepsilon_{y y}\right)\left(\left(y+v(x, y)-O_{y}\right)\left(\sin \theta_{y}\right)+\sin \theta_{y} O_{y}\right) \\
& +\left(\frac{\partial v(x, y)}{\partial x}\right)\left((z)+\left(x+u(x, y)-O_{x}\right)\left(\sin \theta_{y}\right)\right) \\
& \left(1+\varepsilon_{x x}\right)\left((z) \cos \theta_{y}+\sin \theta_{y} O_{x}\right)
\end{aligned}
$$

Interestingly, the perceived strain caused by out-of-plane translation is affine. Further, if we define the relative out-of-plane translation by $f_{z}=(\Delta z) / z$, the perceived shear strain is unchanged and the normal strain becomes:

$$
\varepsilon^{\prime}=\frac{\varepsilon-f_{z}}{1+f_{z}}
$$

The perceived strain when experiencing out-of-plane rotation is more complex as the magnification is not constant for the field of view. Therefore, the magnitude of rotation and the location of the rotation axis are both important parameters in quantifying strain errors. 
The derivations described in this section assume the object translates/rotates out-ofplane; however, there is can be a similar parallax effect caused by misalignment (i.e., rotation) between the scintillator and camera. For best results, both should be mounted on an optical breadboard for precise alignment.

Analytical models for anticipated error caused by out-of-plane motion and contrastto-noise ratio have been developed; however, they must be validated with experimental data before use, and will be in the Ch. 5 . The design of experiments including specimen preparation and DIC methods will be discussed in the next chapter. 


\section{Chapter 5}

\section{Experimental Details}

A longterm objective of this work is to prepare for the implementation of XDIC for cadaveric brain studies at Carleton University. For this reason, many experimental details, parameters, and specimens were selected for their relevance to future trials. A range of X-ray experiments described below were conducted using the general methodology underlined in this chapter, with slight variations in specimen design based on experimental objectives.

\subsection{X-ray Imaging System}

A significant portion of my early thesis work involved the design and selection of components required to set-up the high-speed X-ray facility at Carleton University. Although there was some discussion in Ch. 2 regarding the imaging system methods and requirements, a complete description of the system is provided here.

Commercially available X-ray systems have two sub-components: $\mathrm{X}$-ray generator and image acquisition. The X-ray generator power levels and imaging frame rates vary with diagnostic requirements. For example, imaging rates on the order of 25-30 fps are often sufficient for dynamic diagnostic analyses [25]. As radiography is commonly used for medical diagnostics, care is taken to minimize patient exposure to X-rays. Therefore, unnecessarily high image rates that result in higher patient dose are avoided, meaning faster 
imaging systems are not commercially available.

Uncoupling the optical system into two sub-systems, photon conversion and highspeed imaging, makes significantly higher frame rates $\left(10^{3}-10^{5}\right)$ achievable. These frame rates allow for meaningful analyses of material deformation during dynamic impact events. Additionally, as the analyses are conducted for material characterization, significantly higher exposure doses can be implemented than for patient diagnosis.

The specific components used in our X-ray facility are an $80 \mathrm{~kW}$ tungsten-rhenium rotating anode generator, a thalium-doped cesium iodide scintillator, and a high-speed camera. An $80 \mathrm{~kW}$ X-ray tube was selected to provide sufficient contrast for the expected range of experiments anticipated. A balance between transmission through specimen (high $\mathrm{kV}$ ) and efficient absorption on the scintillator (low $\mathrm{kV}$ ) is required. The CsI(Tl) scintillator has high photon output $(\approx 56-66$ photons $/ \mathrm{keV})$, rapid decay time $(0.6-0.9 \mathrm{~ms} 1 / \mathrm{e}$ time constant), and high X-ray stopping power [66], [23], [67]. The most important feature of the system is the optimal spectral response of the high-speed camera relative to the scintillator output. Both the CsI(Tl) emission spectrum and camera quantum efficiency peak near

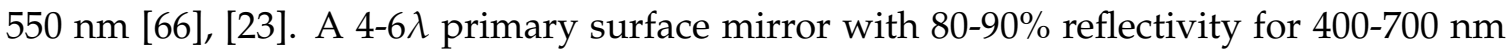
is used to direct scintillator emissions to the camera, placed outside of the direct X-ray beam. Due to proper scintillator emission/quantum efficiency coupling, an image intensifier is not required for this system to generate sufficient light for high-speed applications. The current configuration of the X-ray system including all components described above is shown in Fig. 46. A schematic of the system was shown in the GUI (Fig. 36).

Typical experimental design parameters restrict composition and dimension of the specimen and imaging frame rate. From these requirements, X-ray power levels, record time, field of view, and object placement can be selected. Absorption radiography images a projection of the object rather than the actual surface, therefore it is important to consider the influence of specimen placement relative to the source and scintillator on feature magnification and edge blur, as discussed in Sec. 2.1.4. 


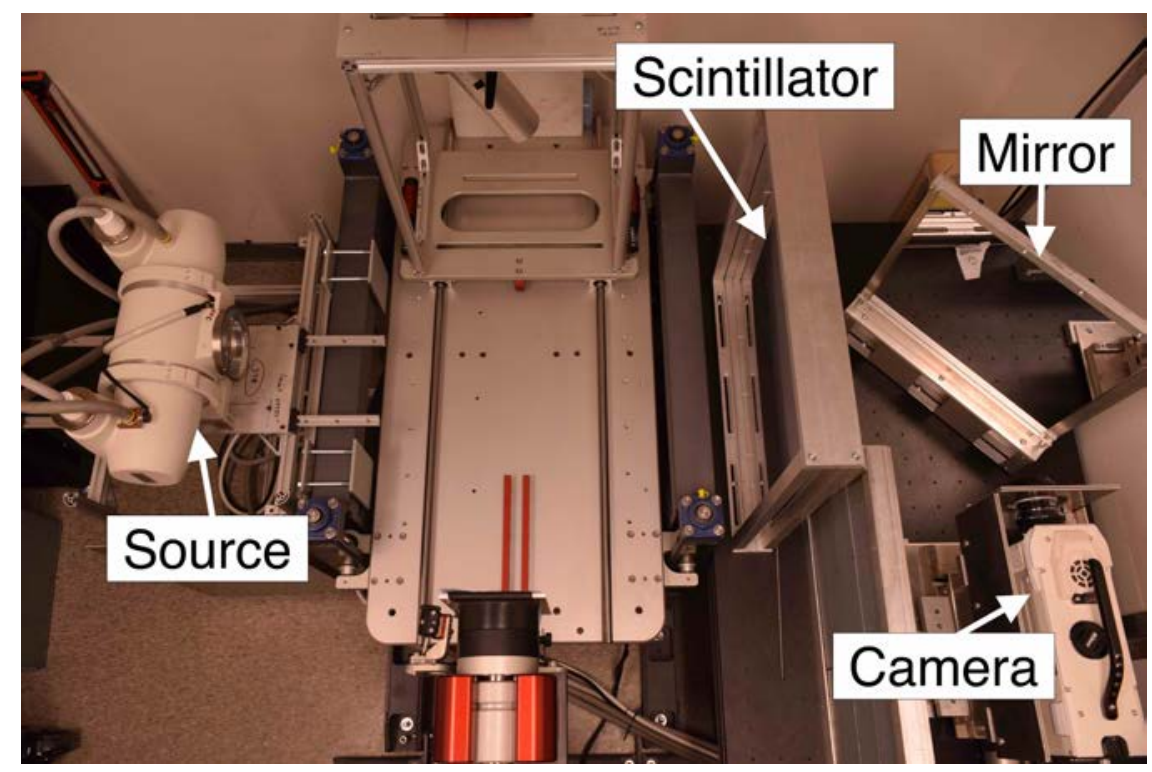

Figure 46: Overhead photograph of the high-speed X-ray system at Carleton University.

\subsection{Calibration}

Variations in image intensity must be isolated to represent the specimen. This is achieved by reducing spatial noise distortions from the system. Black balance and fixed pattern noise may be required. Black balance is first performed by the camera to establish a uniform baseline signal when no light reaches the camera sensor. It is then necessary to correct for fixed pattern noise using a flat-field correction. This process is caused by minute variations in circuitry resulting in slightly different output for each pixel given a specific input. The correction process is equivalent to pixel gain calibration using a series of exposures at different power levels. The resulting correction may reduce the perceived effect of radial intensity variation inherent to X-ray systems as discussed in Sec. 4.1.7.

\subsection{Materials}

The XDIC technique developed as part of this thesis can be implemented either by implanting radiographic contrast markers in existing specimens, or casting specimens such that 
marker integration is an integral step. As my specific work did not require the implantation of contrast markers in pre-cast or biological samples, the discussion below specifically applies to the creation of specimens with markers embedded during casting.

\subsubsection{Tissue Surrogate Materials}

The materials selected for specimen creation were generally selected for their relevance to future PMHS cadaveric brain studies, therefore, elastomers having densities of approximately $1 \mathrm{~g} / \mathrm{cm}^{3}$ or tunable properties were preferred as tissue surrogates. The majority of specimens were composed of polydimethylsiloxane (PDMS) as it has been used in published studies as a tissue surrogate material and its material properties can be finely tuned as required [70]. It has also been used in previous headform studies specifically for the $\mathrm{BI}^{2} \mathrm{PED}$ headform. A commercially produced thermoplastic, Gelatin \# 4 (Humimic Medical, Fort Smith, USA), referred to as "Humimic" for the remainder of this work, was also used as a tissue simulant for its mechanical properties and workability.

\subsubsection{Marker Composition}

As discussed in Sec. 2.3, to generate contrast in X-ray images, a secondary material having different composition and/or density must be embedded in the bulk matrix however, studies by [60] mention the effect of density on dynamic coupling, suggesting contrast is not the only requirement when designing markers.

Therefore, as a less-obtrusive alternative to high-contrast markers such as lead or tin, highly tunable markers composed of the bulk material doped with a specific quantity of barium sulphate were used. Barium sulphate $\left(\mathrm{BaSO}_{4}\right)$ is a widely used medical diagnostic imaging contrast agent due to the high atomic number of barium (56) and density of $4.5 \mathrm{~g} / \mathrm{cm}^{3}$, providing high X-ray attenuation [71]. It is practical for use in our method as it is available in powder form, allowing for easier distribution in brain surrogate materials during the casting phase. Shown in Fig. 47, are the mass and linear attenuation profiles for 


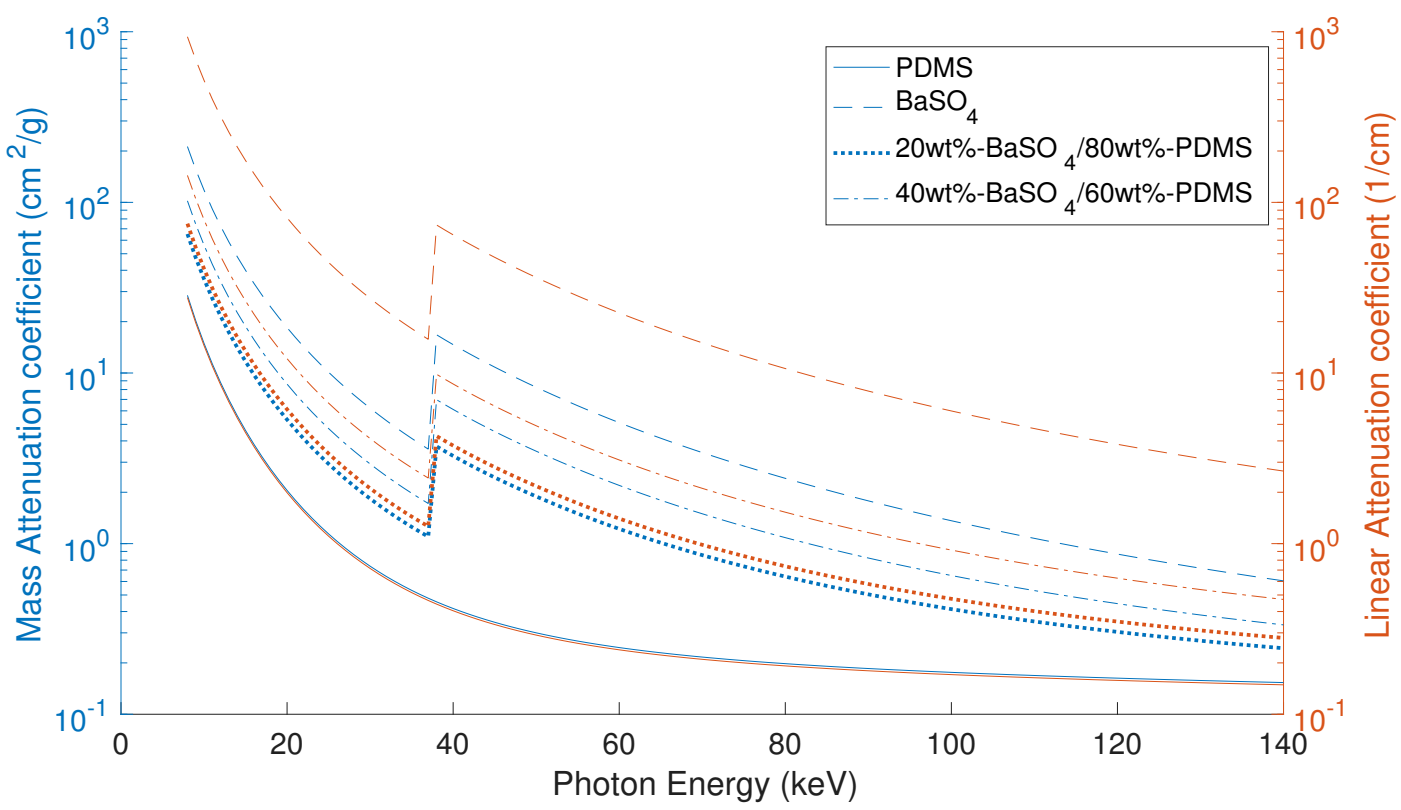

Figure 47: Linear and mass attenuation profile of Barium Sulphate with PDMS at varying concentrations [20].

PDMS mixed with various concentrations of $\mathrm{BaSO}_{4}$.

The effects of increasing $\mathrm{BASO}_{4}$ composition on markers is shown in Fig. 47. The densities of marker compositions in Fig. 47, determined using the rule of mixtures (Eq. 101), is shown in Tab. 5 [61].

\subsubsection{Specimen Preparation}

As the specimens required embedding of contrast markers, the specimens were prepared in several steps. First, a negative mould of the full specimen was created using either rapid prototyped models or silicone cast first as a positive filling a cavity (i.e., skull cavity), then as a silicone negative (i.e., skull lining). Second, the negative mould was partially filled with the bulk material up to the required marker plane. Third, the pre-made contrast markers were layed in place in this internal plane. Immediately following the placement of markers in the target plane, the remainder of the bulk specimen could be cast. The process for casting a specimen remained relatively constant between experiments, however, two 
Table 5: Densities of PDMS-BaSO ${ }^{4}$ Mixtures.

\begin{tabular}{|c|c|}
\hline 100wt \% PDMS & $0.97 \mathrm{~g} / \mathrm{c}$ \\
\hline 90wt $\%$ PDMS-10wt $\% \mathrm{BaSO}_{4}$ & $1.05 \mathrm{~g} / \mathrm{cm}^{3}$ \\
\hline 80wt $\%$ PDMS-20wt $\% \mathrm{BaSO}_{4}$ & $1.15 \mathrm{~g} / \mathrm{cm}^{3}$ \\
\hline $70 w t \%$ PDMS-30wt $\% \mathrm{BaSO}_{4}$ & $1.27 \mathrm{~g} / \mathrm{cm}^{3}$ \\
\hline $60 w t \%$ PDMS-40wt $\% \mathrm{BaSO}_{4}$ & $1.41 \mathrm{~g} / \mathrm{cm}^{3}$ \\
\hline $50 w t \%$ PDMS-50wt $\% \mathrm{BaSO}_{4}$ & $1.60 \mathrm{~g} / \mathrm{cm}^{3}$ \\
\hline $100 \mathrm{wt} \% \mathrm{BaSO}_{4}$ & $4.50 \mathrm{~g} / \mathrm{cm}^{3}$ \\
\hline
\end{tabular}

methods were used for the preparation of contrast markers.

The first method of contrast marker creation consisted of casting sheets of the contrast material (i.e., $\mathrm{PDMS}_{\text {and }} \mathrm{BaSO}_{4}$ ), and cutting approximately evenly sized markers. These markers were then quasi-randomly distributed over the half-cast mould, targetting an optimal surface area coverage for DIC of approximately $50 \%$. In practice, this method proved difficult to implement. Ensuring consistent marker dimensions, area coverage, and spacing proved to be challenging. The markers tended to migrate during the pouring of the second half of the bulk material, influencing their position. Placement of the markers was found to be more accurate if they were positioned before the first layer was fully cured as there was an increase in bonding between bulk specimen and markers. A picture of the markers before embedding in a specimen are shown in Fig. 48.

The second method of contrast marker creation consisted of casting a template of doped material with voids that would create contrast. The mould positive was created using a rapid prototyped model designed with randomly distributed holes having approximately $50 \%$ area coverage. A negative of the slice template was then created using casting silicone. A marker slice cast using this method is layed in place in the half-cast specimen, and the voids are filled during pouring of the second half of the mould. This technique, co-developed by colleague Scott Dutrisac, was my preferred method for future 


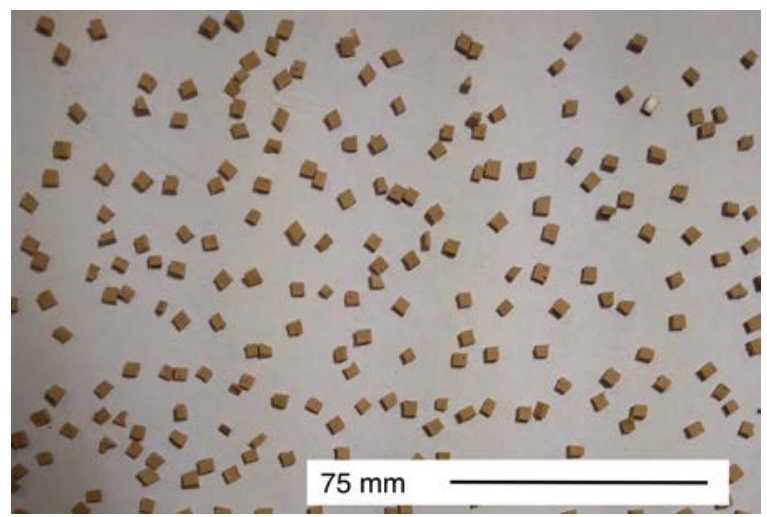

Figure 48: Photograph of radiographic contrast markers (50 wt $\% \mathrm{BaSO}_{4}, 50 \mathrm{wt} \%$ Sylgard 184) before embedding in a specimen

marker integration due to ease of implementation and consistency. A marker slice and corresponding X-ray image after embedding in a specimen are shown in Fig. 49.

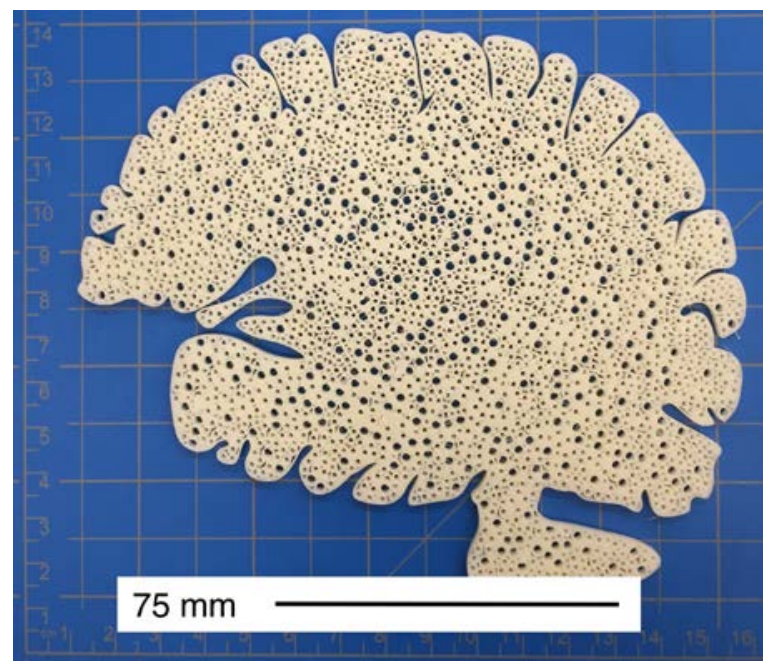

Figure 49: Pre-cast contrast sheet ( $50 \mathrm{wt} \% \mathrm{BaSO}_{4}, 50 \mathrm{wt} \%$ Humimic gel).

A fundamental difference between the two marker creation techniques as discussed above is the apparent contrast. The first method will have dark markers appearing on a light background, while the second method will have a dark background with light markers. Computationally these are equivalent; however, based on the definition of contrast to noise ratio used in Sec. 2.3, light speckles on a dark background are expected to increase CNR and provide higher quality results. 
Specimen preparation varied with the bulk material properties because unique considerations were required when dealing with thermosetting polymers as opposed to thermoplastic polymers. One specific challenge encountered in this context is the thermal loading of markers when pouring the second half of a thermoplastic. The high temperatures required to cast the bulk material can either melt the markers, dispercing their contrast, or heat the previously-poured first half, causing the contrast markers to creep downward. Both of these are challenging as they reduce image quality or planarity of the contrast markers. One method that was found to be successful at preventing these effects in preliminary tests is to pour a thin buffer layer immediately following the markers, and cooling the whole specimen before casting the second phase. This buffer layer is composed of a different thermoplastic with a lower melting temperature than the bulk medium. The second half of the bulk material is subsequently poured to fill the remainder of the mould. The net effect is proper bonding to the marker slice without distortion cuased by the increased temperature. There may be a small effect of the buffer layer on material properties, however, neither this error nor the equivalent error from the embedded marker layer were quantified in this work.

Two types of specimens were used in the analyses described in this paper. The first was a rectangular specimen composed of Humimic gel measuring 150 × 50 × $35 \mathrm{~mm}$ with an embedded pre-case contrast sheet (50 wt $\% \mathrm{BaSO}_{4}, 50 \mathrm{wt} \%$ Humimic). This specimen, used for deformation and out-of-plane measurements is shown in Fig.50.

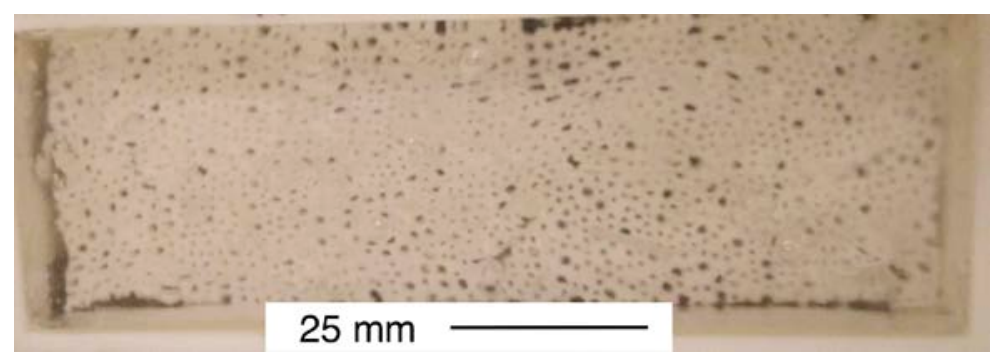

Figure 50: Photograph of the rectangular specimen with embedded contrast slice.

The method was extended to significantly more complex geometry in the Brain Injury 
Protection Evaluation Device ( $\left.\mathrm{BI}^{2} \mathrm{PED}\right)$ headform developped at Defence Research and Development Canada (DRDC) Valcartier and shown here in Fig. 51 [72]. The same three step process, described previously, whereby a pre-cast contrast sheet was placed in a halffull mould before being completely filled was used to cast the $\mathrm{BI}^{2} \mathrm{PED}$ brain. The $\mathrm{BI}^{2} \mathrm{PED}$ brain is PDMS and the contrast sheet used had the same configuration as the rectangular specimen (50 $\mathrm{wt} \% \mathrm{BaSO}_{4}, 50 \mathrm{wt} \%$ Humimic).

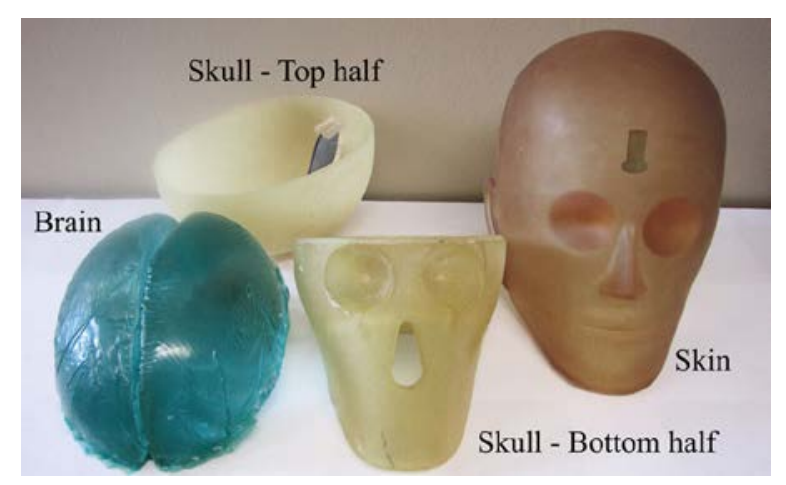

Figure 51: Photograph of the Brain Injury Protection Evaluation Device ( $\left.\mathrm{BI}^{2} \mathrm{PED}\right)$ headform [72].

\subsection{Measurement Method}

The specimen with embedded markers is placed between the source and scintillator for deformation measurements. The source-to-scintillator distance can be adjusted to accomodate experimental requirements (e.g., specimen size, field of view, or magnification). A series of images were recorded during dynamic deformation or in steps for quasi-static analyses, with recording parameters and X-ray exposure determined based on experimental requirements and specimen size.

Preliminary impact experiments conducted used a NOCSAE twin-wire drop apparatus as shown in Fig. 52. Specimens and headforms were mounted to a drop carriage that slides down two parallel wires. A Linear Impact Testing Machine (Cadex, Québec, Canada), 


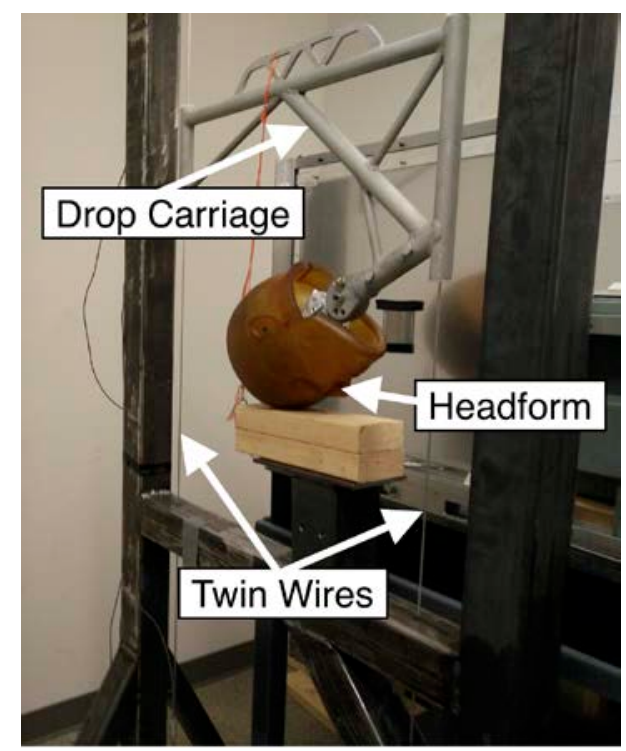

Figure 52: Photograph of the NOCSAE drop used for preliminary impact experiments.

shown in Fig. 53(a), is now used to impact specimens at speeds of 3-10 m/s using a freeflying $13 \mathrm{~kg}$ mass. The linear impactor has a built-in specimen mounting table for precise in- and out-of-plane rotation and translation. The specimen location can be prescribed to ensure proper alignment with the impactor using the mount shown in Fig. 53(b).

Fixtures can be mounted to the impact table as required for quasi-static or dynamic experiments. Quasi-static deformation can either be achieved using a tension/compression apparatus, or by moving the specimen in- or out-of-plane, changing the magnification.

\subsection{Digital Image Correlation Software}

After the collection of a series of images using the in-house X-ray system, displacement and strain fields are then determined using standard DIC software. Although there are many available software packages that are mathematically quite similar, Ncorr open-source DIC software for Matlab (Natick, USA) was used for all analyses [73]. Ncorr was selected due to the open-source and easily edited code allowing for user modifications to automate large, parametric studies. 


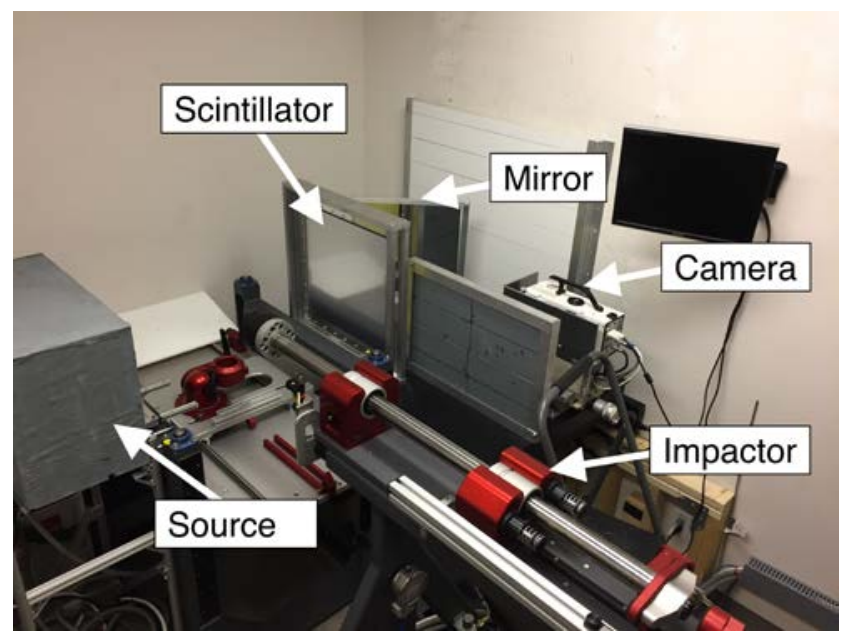

(a)

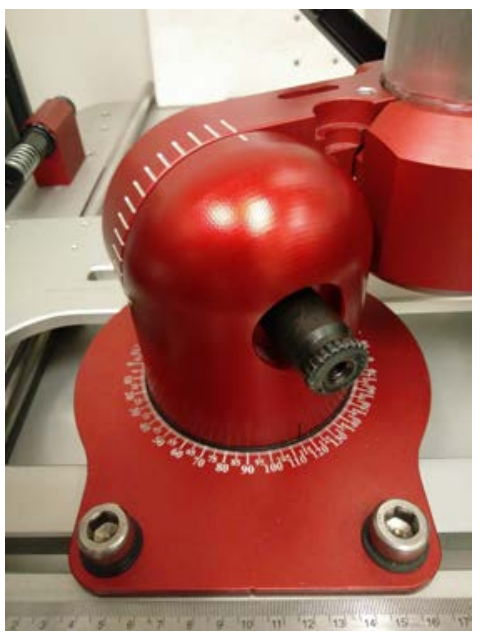

(b)

Figure 53: Photographs of (a) the Cadex Linear Impactor in our facility, and (b), the three degree of freedom mounting fixture.

The displacement and strain plots generated by Ncorr can also be readily modified to express results. A unitless, representative example of output displacement plots for a simple two-image study, where the specimen shown in Fig. 50 was imaged, translated out of plane then imaged again, are shown in Fig. 54(a) and 54(b).

The corresponding displacement fields are shown in Fig. (c) and (d). For better representation of displacement fields, a Matlab script was written to transform displacement surface plots into more easily interpreted quiver plots as shown for the same deformation fields in Fig. 55.

In summary, the experimental procedure consists of specimen preparation, including embedded contrast markers, imaging of dynamic loading (e.g., drop or impact), and DIC displacement/strain analysis. Next, the methodology described in this chapter will be implemented in Ch. 6 to validate analytical models developed in Ch. 4. 


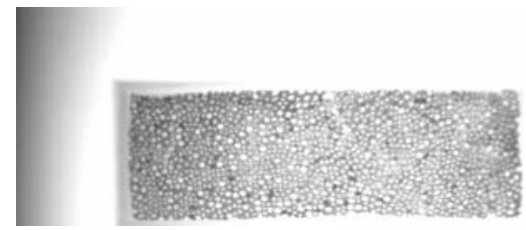

(a)

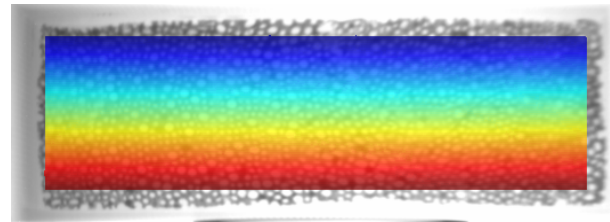

(c)

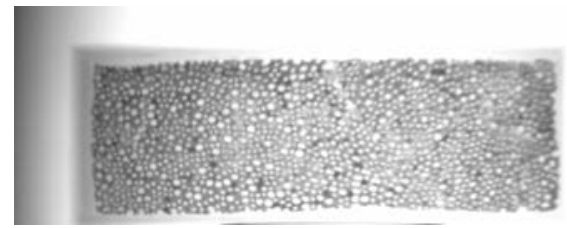

(b)

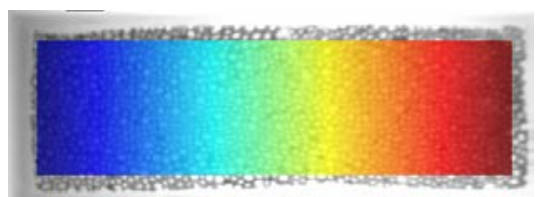

(d)

Figure 54: (a) Original, and (b) of out-of plane translated specimen with perceived displacement plots created by Ncorr DIC software in the (c) vertical; and, (d) horizontal directions.

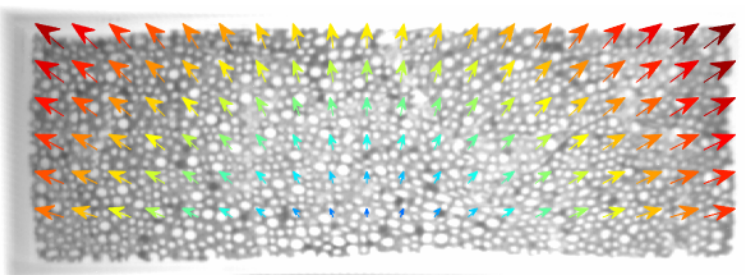

Figure 55: Quiver plots created from Ncorr displacements plots in Fig. 54(c) and $54(d)$ 


\section{Chapter 6}

\section{Results and Discussion}

Both image contrast and out-of-plane motion were previously identified as factors influencing DIC accuracy. This chapter includes discussion on quantifying these errors, validating the analytical model created in Ch. 4, and a preliminary study of surrogate brain deformation in a headform.

\subsection{Out-of-Plane Motion}

An analytical model of DIC strain error caused by out-of-plane motion was developped in Sec. 4.2, with the complete derivation in Appendix B. The model relates perceived strain to applied strain for a strain element experiencing first order deformation, in-plane rotation, two out-of-plane rotations and out-of-plane translation.

\subsubsection{Method}

A specimen composed of Humimic Medical Gel \# 4 with a pre-cast contrast sheet, made of $50 \mathrm{wt} \%-\mathrm{BaSO}_{4}-50 \mathrm{wt} \%$-Humimic \# 4, was used for out-of-plane motion experimental validation. The specimen, shown in Fig. 50, has dimensions of $150 \times 50 \times 35$ mm, with the $3 \mathrm{~mm}$, mid-plane contrast layer embedded from approximately 17 to $19 \mathrm{~mm}$.

The specimen was mounted to the Cadex impactor fixture (Fig. 53(b)), and moved 
and/or rotated out-of-plane. Two sets of experiments were performed: 1) pure out-ofplane translation, 2) pure out-of-plane rotation.

For translation experiments, the specimen was originally located at $615 \mathrm{~mm}$ from the source, and incrementally translated $20 \mathrm{~mm}$ towards the source, resulting in increased magnification, with relative displacements of $20 \mathrm{~mm} \mathrm{(-3.25 \% ),} 40 \mathrm{~mm}(-6.51 \%), 60 \mathrm{~mm} \mathrm{(-}$ $9.76 \%), 80 \mathrm{~mm}(-13.0 \%)$, and $100 \mathrm{~mm}(-16.3 \%)$. The scintillator was located at $990 \mathrm{~mm}$ from the focal spot.

The rotation experiments were similarly performed by placing the specimen and at $619 \mathrm{~mm}$ from the source, with the scintillator at $957 \mathrm{~mm}$. The specimen was rotated counter-clockwise about a point near its centre in $2.5^{\circ}$ increments up to $22.5^{\circ}$.

\subsubsection{Results}

\section{Out-of-Plane Translation}

The analytical model suggests the following strains will be perceived when experiencing pure out-of-plane translation:

$$
\begin{aligned}
& \varepsilon_{x x}^{\prime}=z \frac{\left(1+\varepsilon_{x x}\right)}{(z+\Delta z)}-1=\frac{\left(1+\varepsilon_{x x}\right)}{\left(1+f_{z}\right)}-1=\frac{\left(\varepsilon_{x x}-f_{z}\right)}{\left(1+f_{z}\right)} \\
& \varepsilon_{y y}^{\prime}=z \frac{\left(1+\varepsilon_{y y}\right)}{(z+\Delta z)}-1=\frac{\left(1+\varepsilon_{x x}\right)}{\left(1+f_{z}\right)}-1=\frac{\left(\varepsilon_{x x}-f_{z}\right)}{\left(1+f_{z}\right)}
\end{aligned}
$$

Experimentally, the strain fields in Fig. 56(a) and 56(b) were found for $\varepsilon_{x x}^{\prime}$ and $\varepsilon_{y y}^{\prime}$ when the objects were translated out-of-plane by $-3.25 \%$. The average normal strains were determined for each vertical column in the region of interest. The average $\varepsilon_{y y}^{\prime}$ are plotted in Fig. 57(a), along with the predicted values from Eq. 138. Similarly, Fig. 57(b) has experimental data for $\varepsilon_{y y}^{\prime}$ and analytical data from Eq. 139. The corresponding error between measured and predicted perceived strain are shown in Fig. 58(a) and Fig. 58(b). The DIC 


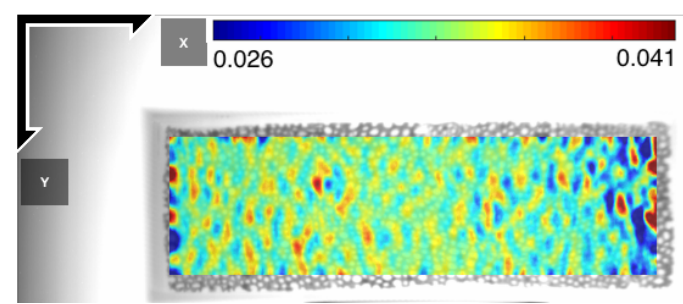

(a)

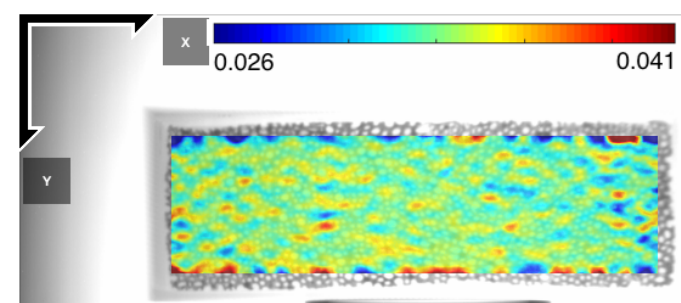

(b)

Figure 56: DIC strain fields from pure out-of-plane translation by $-3.25 \%$ : (a) $\varepsilon_{x x}$ and (b) $\varepsilon_{y y}$. The colourmap is centred about the predicted value, with $\pm 20 \%$ as the bounds.

Table 6: Parameters used in DIC out-of-plane translation analysis

\begin{tabular}{c|c} 
Image Type & $\begin{array}{c}\text { Experimentally acquired } \\
\text { Nikkor f/1.2, 50mm }\end{array}$ \\
Reference ROI dims (pixels) & $1480 \times 468$ \\
Applied Deformation & Out-of-plane translation \\
Speckle Radius (pixels) & $10-15$ \\
Subset Radius (pixels) & 25 \\
Strain Radius (pixels) & 5 \\
Subset Spacing (pixels) & 0 \\
Seeds & 1 \\
DIC Software & Ncorr [73] \\
Interpolation Method & Biquintic B splines
\end{tabular}

parameters used in this analysis are shown in Tab. 7.

\section{Out-of-Plane Rotation}

The analytical model developed previously defines the perceived strains caused by pure rotation about the $y$ axis as the following: 


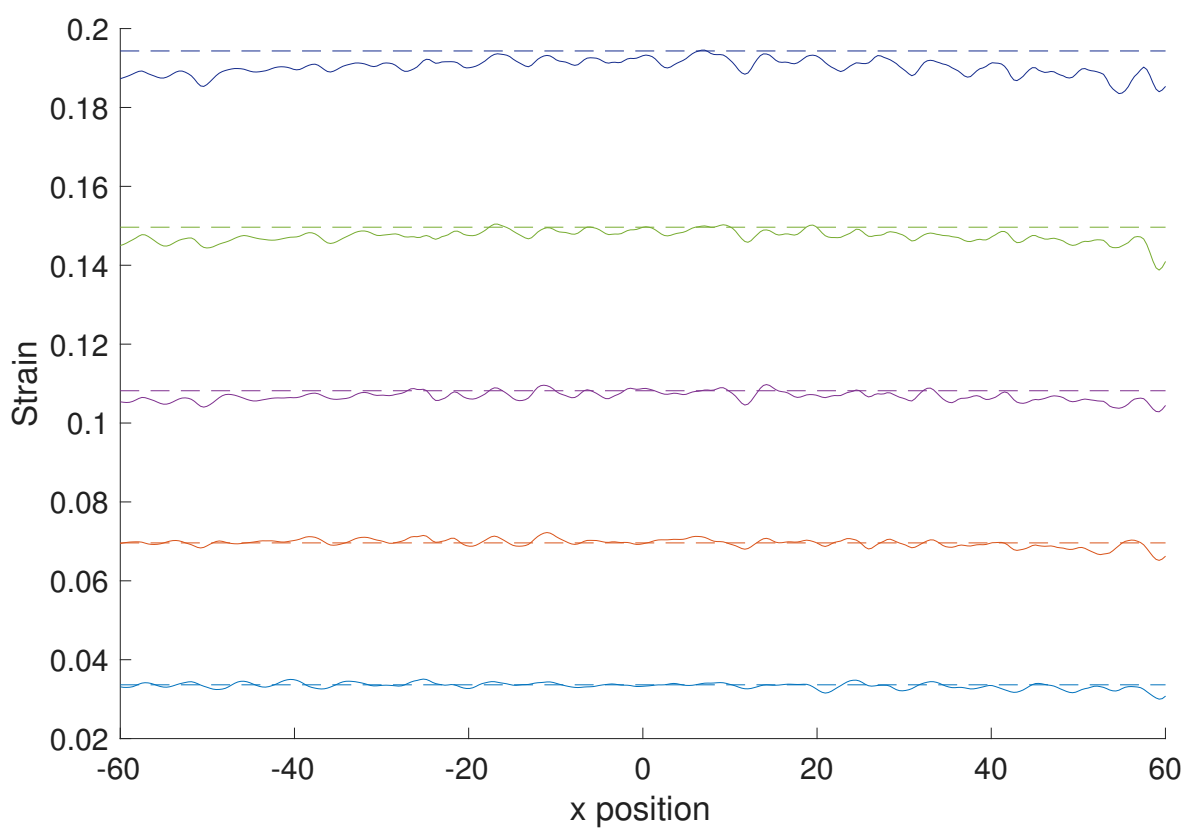

(a)

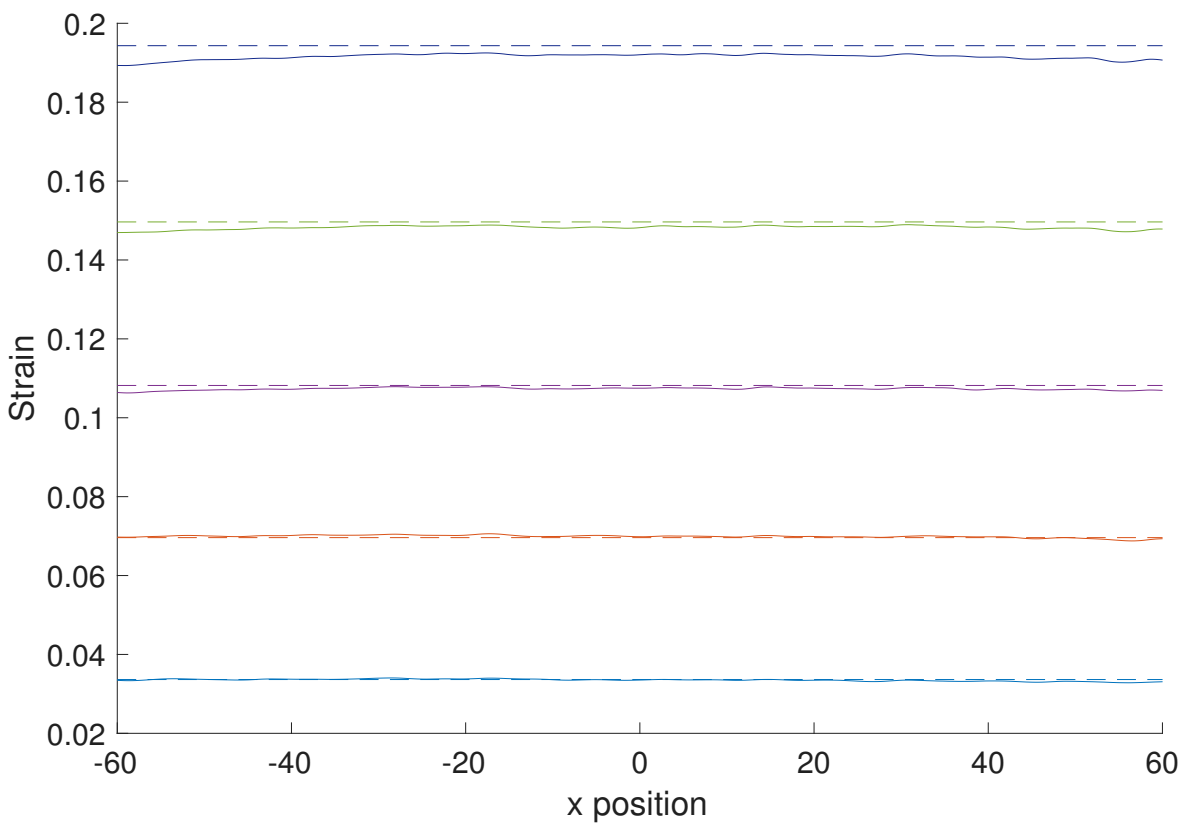

(b)

Figure 57: Average measured and predicted values of perceived strains caused by out-of-plane translation along $x$ of the specimen: (a) $\varepsilon_{x x}$ and (b) $\varepsilon_{y y}$ 


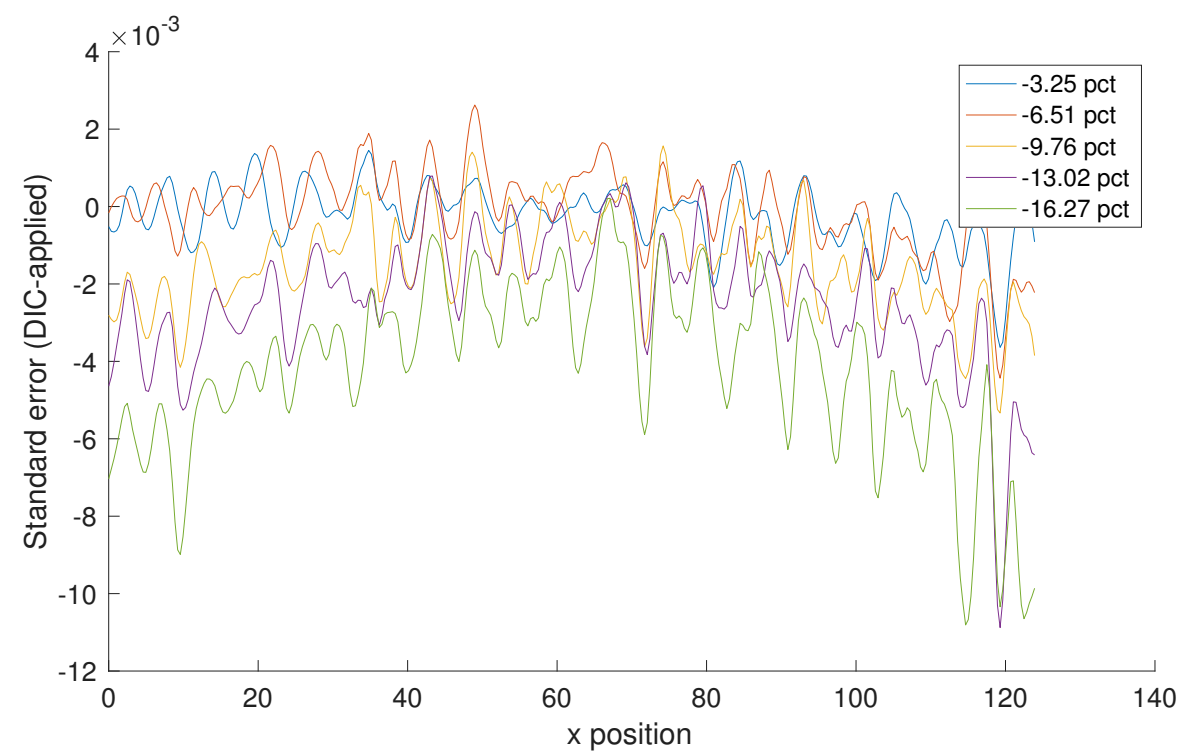

(a)

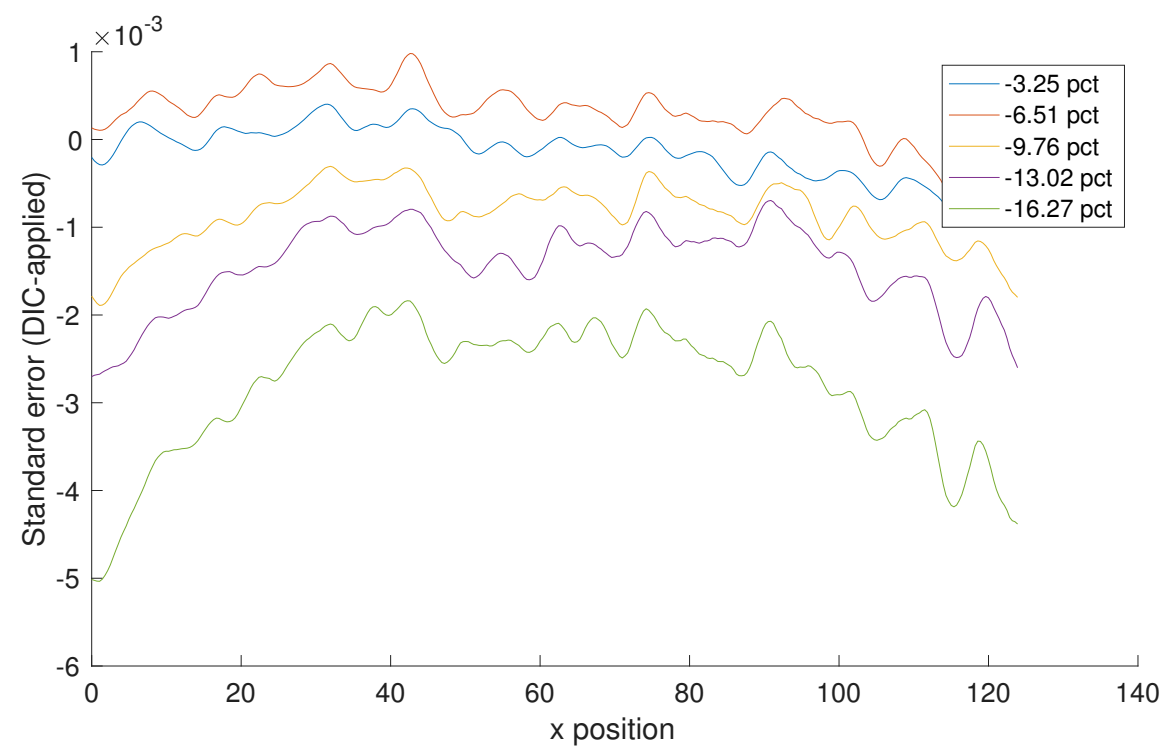

(b)

Figure 58: Residual standard error of perceived strains (measured - predicted) for various relative out-of-plane translation by $x$ position along the specimen: (a) $\varepsilon_{x x}$ and (b) $\varepsilon_{y y}$ 


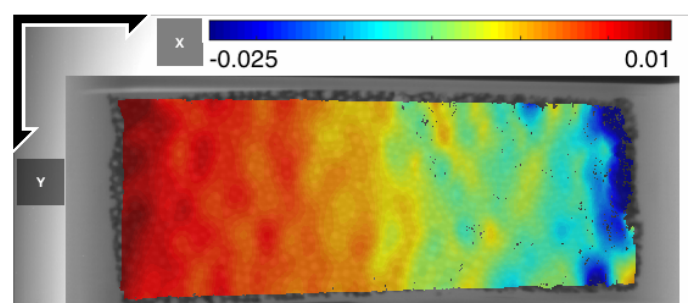

(a)

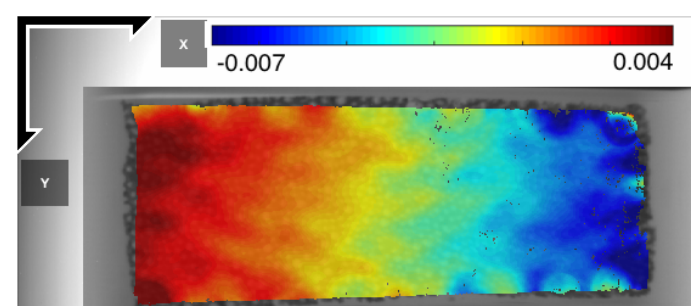

(b)

Figure 59: DIC strain fields for out-of-plane rotation by $2.5^{\circ}$ : (a) $\varepsilon_{x x}$ and (b) $\varepsilon_{y y}$.

$$
\begin{gathered}
\varepsilon_{x x}^{\prime}=z \frac{\left(1+\varepsilon_{x x}\right)\left((z) \cos \theta_{y}+\sin \theta_{y} O_{x}\right)}{\left(z-\sin \theta_{y}\left(x+u_{1}-O_{x}\right)\right)^{2}}-1 \\
\varepsilon_{y y}^{\prime}=z \frac{\left(\frac{\partial u_{1}}{\partial y}\right)\left(y+v_{1}\right)\left(\sin \theta_{y}\right)}{+\left(1+\varepsilon_{y y}\right)\left((z)-\left(x+u_{1}-O_{x}\right)\left(\sin \theta_{y}\right)\right)}-1 \\
\left(z-\sin \theta_{y}\left(x+u_{1}-O_{x}\right)\right)^{2}
\end{gathered}
$$

Experimentally, the strain fields in Fig. 59(a) and 59(b) were found for $\varepsilon_{x x}^{\prime}$ and $\varepsilon_{y y}^{\prime}$ when the objects were translated out-of-plane by $2.5^{\circ}$. The DIC parameters used in this analysis are shown in Tab. 7.

The average strains in the $x$ and $y$ directions were determined for each vertical column in the region of interest. The average $\varepsilon_{y y}^{\prime}$ are plotted in Fig. 60(a), along with the predicted values from Eq. 140, shown here for rotations of $5^{\circ}, 10^{\circ}, 15^{\circ}$, and $20^{\circ}$. Similarly, Fig. 60(b) has experimental data for $\varepsilon_{y y}^{\prime}$ and analytical data from Eq. 141. The difference between predicted and measured strains based on perceived deformation from out-of-plane rotation is shown in Fig. 61(a) and Fig. 61(b). 


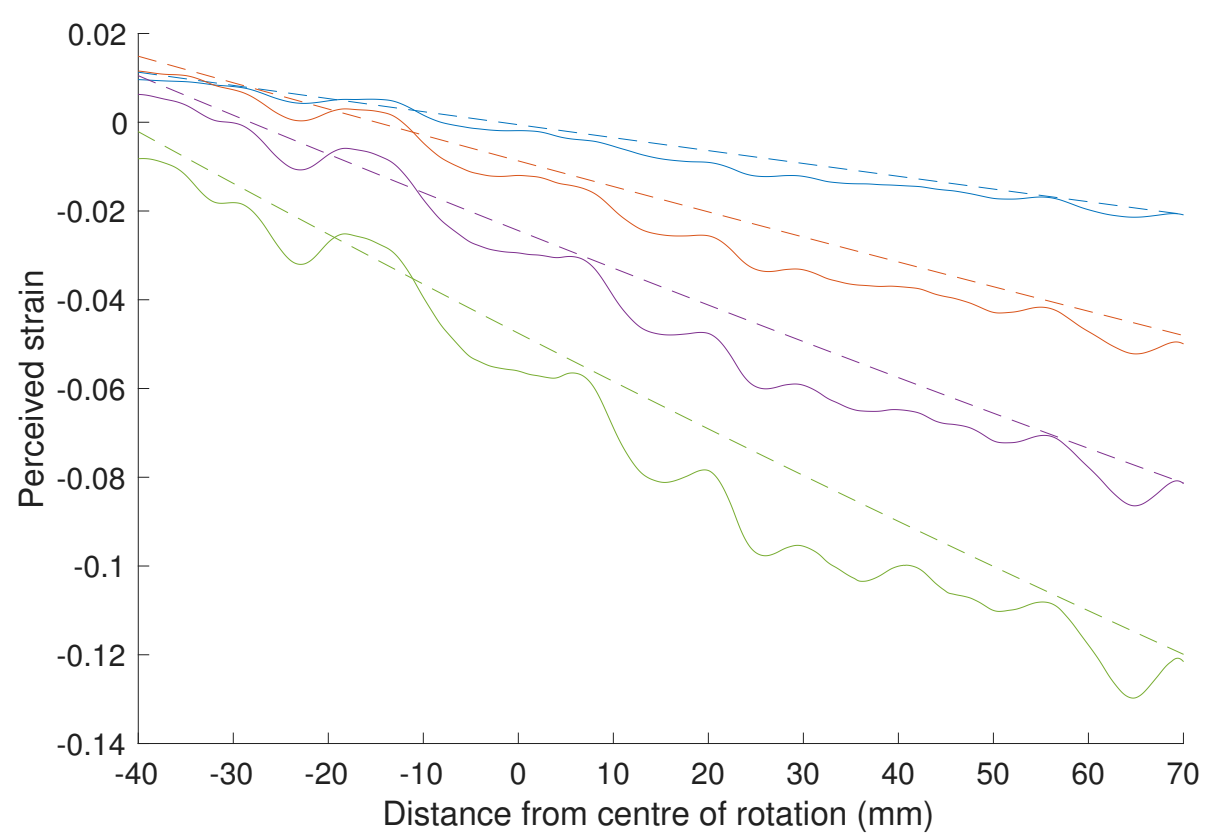

(a)

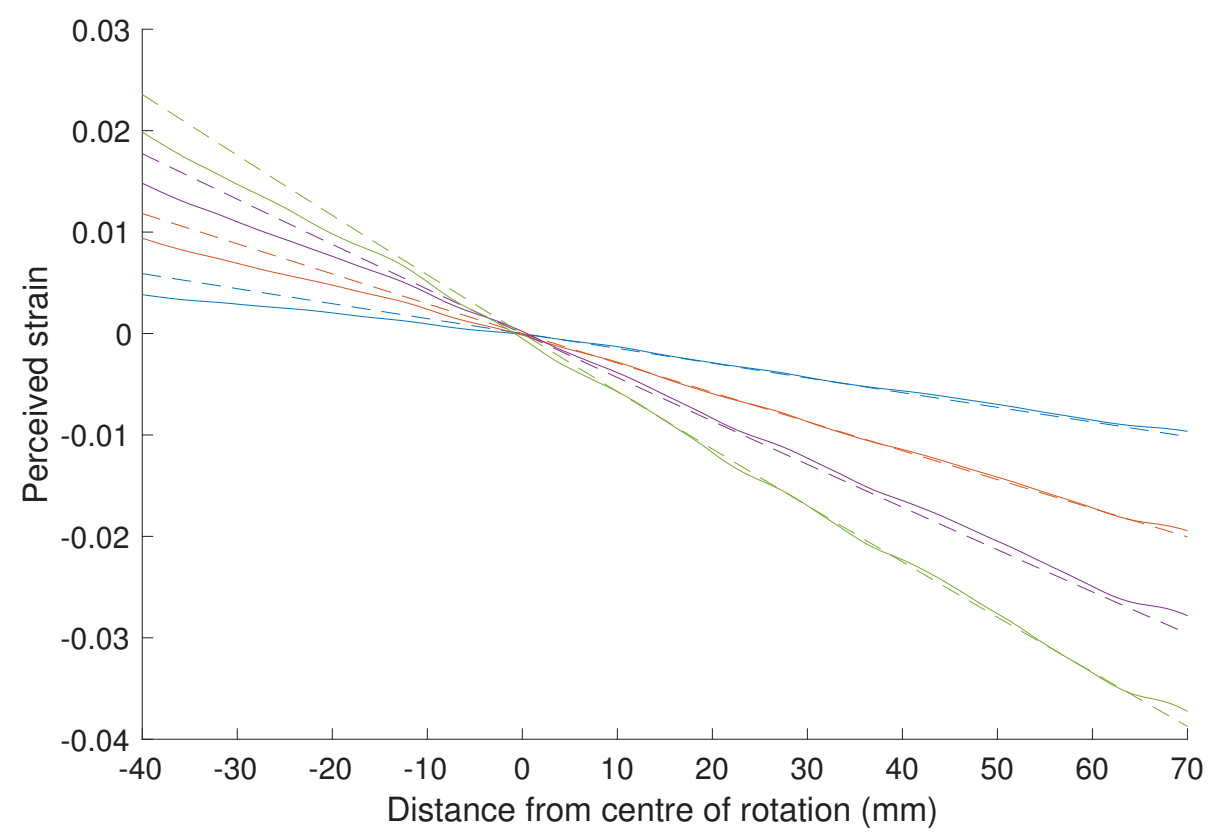

(b)

Figure 60: Average measured and predicted values of perceived strains caused by out-of-plane rotation about the vertical axis along $x$ length of the specimen: (a) $\varepsilon_{x x}$ and (b) $\varepsilon_{y y}$ 


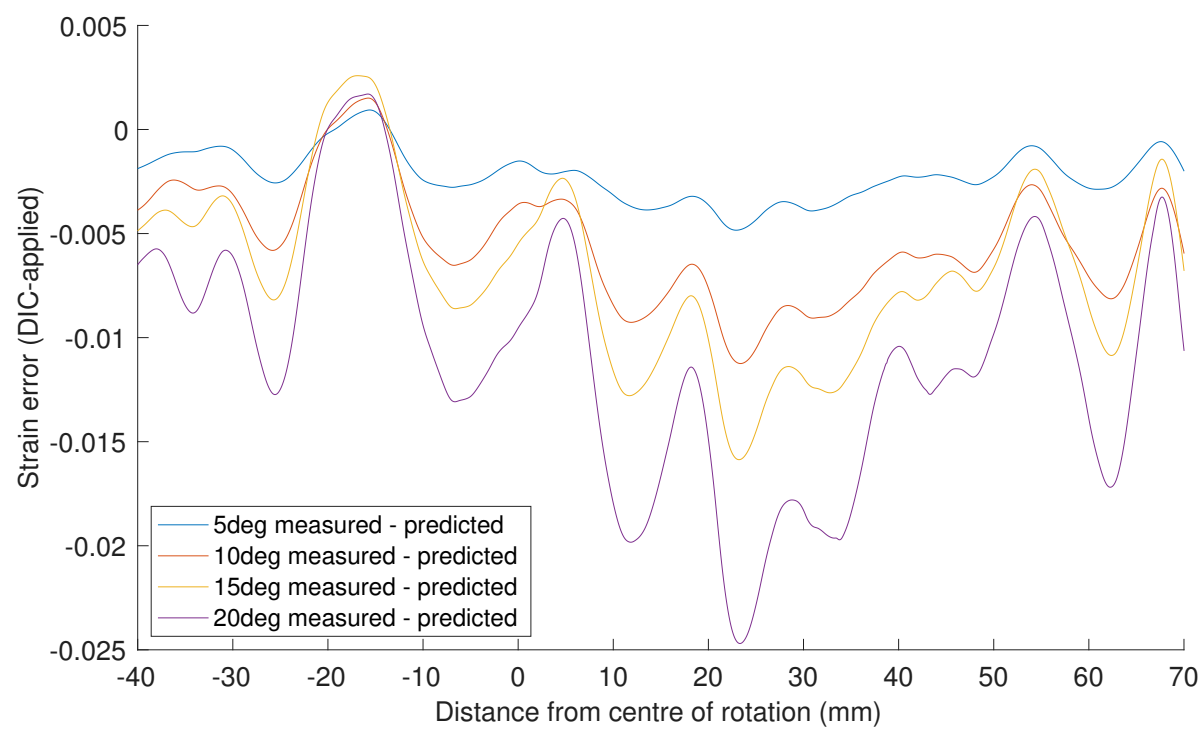

(a)

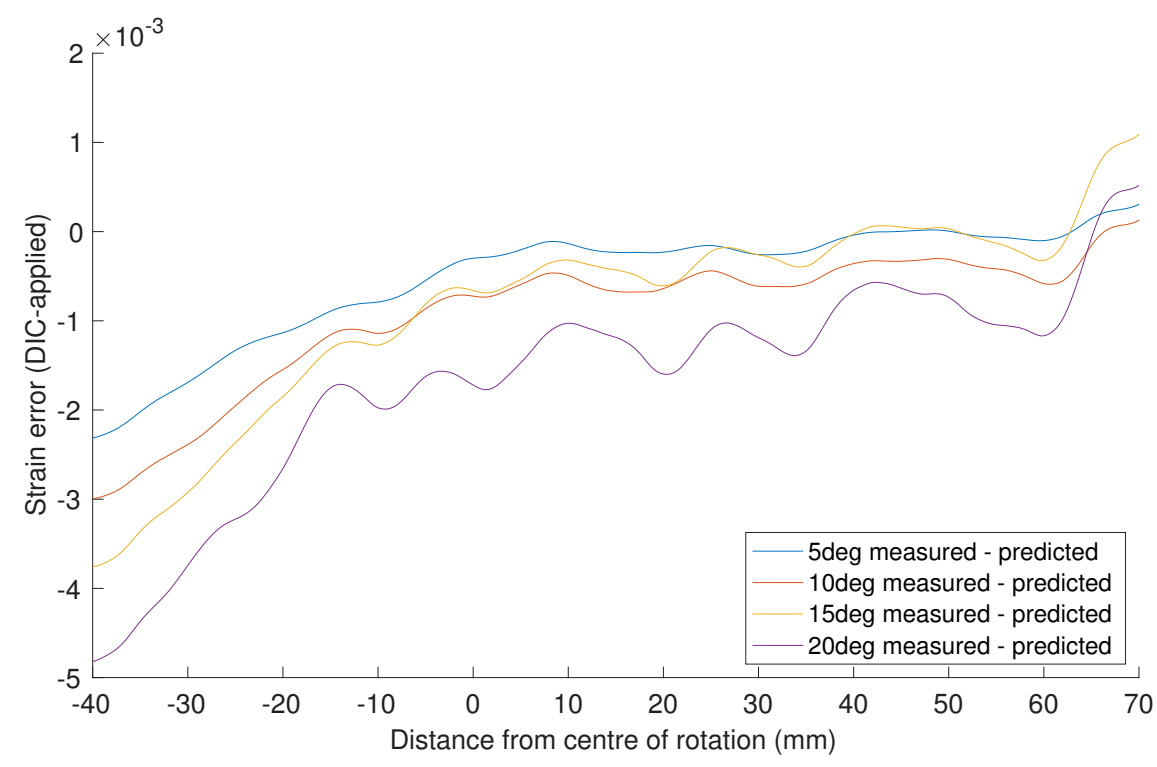

(b)

Figure 61: Residual standard error perceived strains (measured - predicted) for various relative out-of-plane rotations about the vertical axis along the $x$ position of the specimen: (a) $\varepsilon_{x x}$ and (b) $\varepsilon_{y y}$ 
Table 7: Parameters used in DIC out-of-plane rotation analysis

\begin{tabular}{c|c} 
Image Type & $\begin{array}{c}\text { Experimentally acquired } \\
\text { Nikkor f/1.2, 50mm }\end{array}$ \\
Reference ROI dims (pixels) & $1653 \times 466$ \\
Applied Deformation & Out-of-plane rotation \\
Speckle Radius (pixels) & $10-15$ \\
Subset Radius (pixels) & 25 \\
Strain Radius (pixels) & 5 \\
Subset Spacing (pixels) & 0 \\
Seeds & 1 \\
DIC Software & Ncorr [73] \\
Interpolation Method & Biquintic B splines
\end{tabular}

\subsubsection{Discussion}

The experiments performed for validation of the analytical out-of-plane motion model included both pure translation and pure rotation of a specimen containing a $3 \mathrm{~mm}$ contrastgenerating slice. Example strain fields, shown in Fig. 56(a) and Fig. 56(b), are not completely smooth; however, the majority of the strain fields are within 0.05 of the expected value (green).

Before comparing experimental to analytical data, several observations can be made of the trends. The perceived strain increases linearly with out-of-plane translation, with the same effect in $x$ and $y$ for an applied strain. The translation varies the linear projection of the whole specimen on the scintillator, resulting in a uniform magnification change.

Out-of-plane rotation analyses also show interesting trends. For example, the location of zero perceived strain in $x$ (i.e., $\varepsilon_{x x}=0$ ) is dependent upon rotation magnitude. Further, it is shown that the error increases with distance from the centre of rotation, corresponding to a larger effective out-of-plane translation. The centre of rotation has a perceived strain ( $\epsilon_{y y}$ of zero, which is consistent with the 2D model and validation performed by [15] for optical DIC. The centre of rotation does not move out of plane, and therefore should not 
change magnification in $y$, explaining the intercept. It is also shown that the error caused by out-of-plane motion will be significantly more important in the direction normal to the rotation axis.

Although the equations derived for the case of simple rotation are not identical to the solution proposed by [15], and described in Sec. 3.2.3, the solution provided in this work was derived for the general case. The previous model assumed rotation about $x=y=$ 0 , was restricted to a 1D body, and rotation and translation were derived independently when real world applications are likely to encounter both simultaneously. The 1D strain equations can be obtained from the equations derived in this work. For example, with the the following simplifications it is possible to obtain the same solution as [15] for $\varepsilon_{y y}$.

$$
\begin{aligned}
\varepsilon_{y y}= & \frac{z}{z-\left(x-O_{x}\right) \sin \theta}-1 \approx \frac{z+\left(x-O_{x}\right) \sin \theta}{z}-1=\frac{\left(x-O_{x}\right) \sin \theta}{z} \\
& =-\frac{Y \sin \theta}{Z}(\text { after changing notation to be compatible with [15] })
\end{aligned}
$$

The average value in each vertical column in the region of interest (56(a) and 56(b)) was used to generate Fig. 57(a) and Fig. 57(b). The perceived strain predicted by the analytical model are also shown for relative displacements of $-3.25 \%$ to $-16.3 \%$. The analytical model is shown to predict the measured strains in both the $x$ and $y$ directions to within 0.005 when the relative translation was less than 10\% (Fig. 58(a) and Fig. 58(b)) Strain errors caused by small out-of-plane translations are most well described by the analytical model. After large translations, the model appears to over-predict the errors, with the error increasing further from the centre of the specimen.

A similar experiment was conducted for out-of-plane rotation, with strain plots shown in Fig. 59(a) and Fig. 59(b). The corresponding analytical and experimental results are shown in Fig. 60(a) and Fig. 60(b), with differential errors shown in 61(a) and Fig. 61(b). The prediction for $\varepsilon_{y y}$ was very accurate, specifically for the portion of the specimen furthering itself from the source (erros; 0.005 ) for rotations up to $20^{\circ}$. There is a noted deviation between measured and predicted strain for the portion of the specimen rotating 
towards the source. The error in predicted versus measured perceived $\varepsilon_{x x}$ was more significant. Although there was significant variation in the results, the analytical model was able to predict trends in the data. Variations between the two methods increased with rotation angle.

An experimental factor hypothesized to play an important role in discrepancies between calculated and predicted strain is the contrast layer thickness. The model derivation assumed a zero-thickness contrast layer as is the case with optical DIC speckles; however, a $3 \mathrm{~mm}$ contrast layer is sufficient to cause blurring around the speckles. The magnitude of the blurring effect increases with magnification, therefore it is expected that out-of-plane motion towards the source will cause greater discrepancy between the model and experimental results, as seen in $\varepsilon_{y y}$ for rotation. Additionally, the differences for $\varepsilon_{x x}$ in rotation could be caused by improper contrast layer placement. It is possible that the contrast layer was not planar in the specimen. This hypothesis is supported by the similar profile of experimental results at different rotations, and would also explain why $\varepsilon_{x x}$ saw larger errors than $\varepsilon_{y y}$.

In practice, out-of-plane motion should be minimized. A threshold for allowable translation and rotation should be determined by the user based on allowable measurement uncertainties. For example, when out of plane translation occurs, the error in normal strains can be rapidly approximated as:

$$
\varepsilon^{\prime} \approx \varepsilon-f_{z}
$$

As strain increases, the error will be less important, therefore, allowable relative out-ofplane translation should be much smaller than the expected range of calculated strains. Out-of-plane rotation can also be approximated using a reduced equation. The strain parallel to the axis of rotation can also be rapidly approximated by the following:

$$
\varepsilon^{\prime} \approx \varepsilon-f_{z}=\varepsilon-\frac{x \sin \theta}{z}
$$


After an allowable experimental strain error is determined, a maximum out-of-plane rotation can be determined based on the expected centre of rotation and specimen length.

Based on the magnitude of expected errors in strain caused by out-of-plane motion, it is highly recommended to use a stereoscopic image acquisition system whenever possible. It is understood that this may not always be feasible, long-term planning for facility upgrades should include a second X-ray system. In the interrim, a series of reference figures were produced to cover the experimental error expected in a wide range of experimental situations. These plots are designed to be quick-reference guides for users to quantify uncertainty based on approximate knowledge of out-of-plane motion. The reference figures are provided in Appendix C.1.

\subsection{Analytical CNR Tool Validation}

DIC measurement rates are limited by the temporal resolution of the image acquisition system. If the captured images are free of motion blur and have sufficient contrast with low noise, the software will compute correct deformation and strain fields. Optical DIC measurements require a high contrast, patterned surface, typically black speckles on a white surface. In this configuration, it is possible to perform DIC analyses at incredibly high frame rates; for example, the Hypervision HPV-X2 (Shimadzu Corporation, Japan) is able to record at $10^{7} \mathrm{fps}$. Contrarily, in XDIC, it is not always feasible to increase the contrast due to focal spot power flux limits, required imaging rate or specimen dimensions. Therefore, in dynamic experiments $\left(10^{3}-10^{4} \mathrm{fps}\right)$, resulting images do not always achieve high contrast. For example, a headform with embedded markers will have a greyscale projection for the brain surrogate material, with darker speckles created by X-ray markers at high frame rates, which is significantly different than the black and white images captured optically. However, it should be noted that X-ray is used to image optically inaccessible planes such as inside of a skull that would be impossible using optical techniques. As contrast is not a typical concern with optical DIC, it is necessary to understand the effect of contrast-to-noise ratio on DIC accuracy. The analytical model described in Sec. 4.1 was 
designed for this purpose.

Repeatedly creating radio-opaque markers using trial-and-error to achieve sufficient contrast for DIC accuracy is not a feasible method, especially for cadaveric studies. The general method seen in Sec. 3.3.2 was to use very high contrast materials, typically lead or an equivalent for markers; however, in dynamic studies, this is not a suitable approach as material coupling could dominate measurements. Therefore, the purpose of the method developed herein is to design markers that are minimally invasive while providing sufficient contrast for accurate measurement. The analytical model should provide an equivalent error estimation to the physical process, given a potential specimen definition as described in a flowchart (Fig. 62). If the experimental error and predicted error are equivalent, then the analytical model may be used to test a range of specimen-marker pairs to evaluate their potential use prior to sample creation.

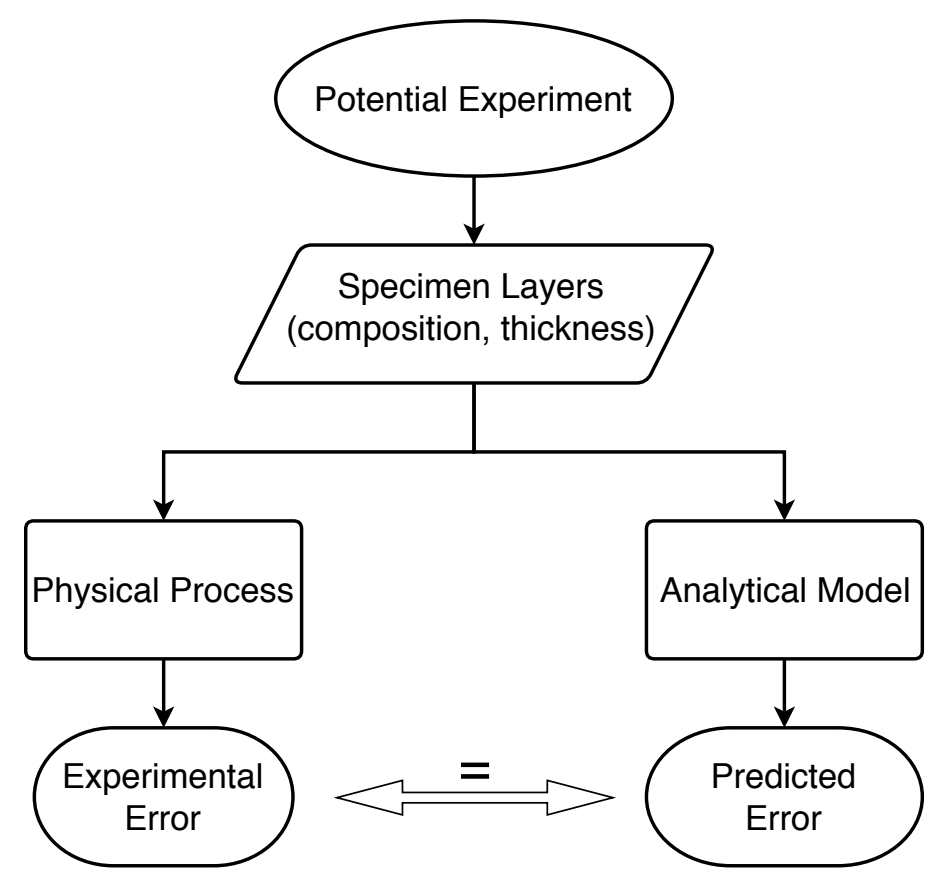

Figure 62: Flowchart demonstrating the purpose of the analytical model. 


\subsubsection{CNR DIC Error}

The first step required in implementing the flowchart shown in Fig. 62 is to quantify the effect of contrast-to-noise ratio on the accuracy of computed DIC strain fields. The flowchart is expanded to include an error function relating CNR to measurement error (Fig. 63). This error function is generally used to determine the anticipated DIC strain error based on a CNR input. Therefore, it is the same function for both the physical process and analytical model.

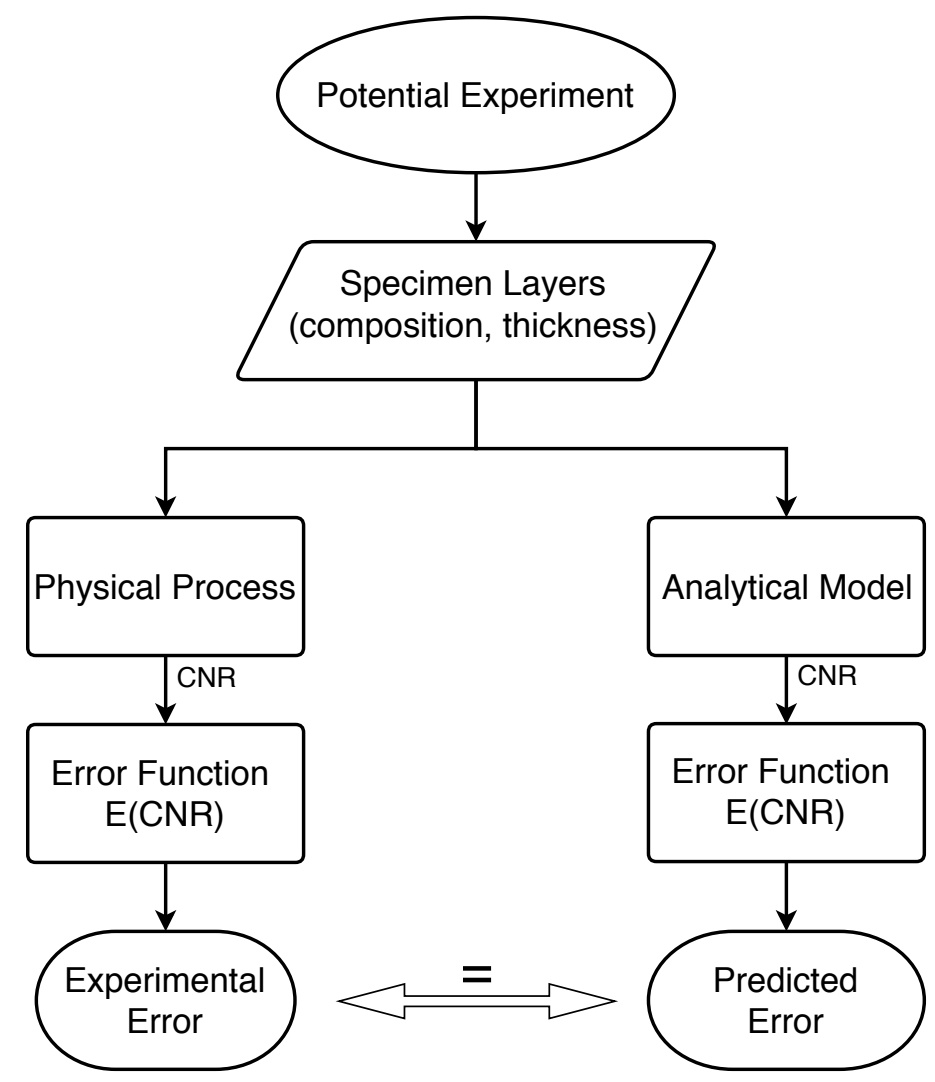

Figure 63: Flowchart demonstrating the application of the analytical model.

\section{Error Function: Method}

To quantify DIC measurement accuracy as a function of CNR, the analysis of a series of synthetic images created in Matlab was performed. The speckled images are similar in design to those used in [42]. The series of 300x300 pixel, speckled images were deformed 
Table 8: Single-pixel CNR for artificial images of set marker and background intensities

\begin{tabular}{c|cccc} 
& \multicolumn{5}{|c}{ Marker Intensity } \\
\hline & 13 & 26 & 38 & 51 \\
\hline 26 & 2.6 & - & - & - \\
38 & 4.1 & 1.9 & - & - \\
51 & 5.3 & 3.5 & 1.8 & - \\
63 & 6.4 & 4.8 & 3.3 & 1.6 \\
77 & 7.3 & 5.8 & 4.4 & 3.0 \\
89 & 8.1 & 6.7 & 5.4 & 4.0 \\
102 & 8.8 & 7.5 & 6.3 & 5.1 \\
115 & 9.5 & 8.3 & 7.2 & 6.0 \\
128 & 10.2 & 9.0 & 8.0 & 6.8
\end{tabular}

to $5 \%, 10 \%$ and $15 \%$ engineering strain with a Poisson's ratio of 0.33 . The 8 -bit images had background intensity values ranging from 26 to 128 and marker intensities of 13 to 51 . Poisson noise was added to represent the shot noise caused by the finite number of photons reaching the camera sensor. This resulted in a series of images having single-pixel CNR given by the Table 8 , where CNR is defined here as the difference between marker and background specimen pixel values $\left(P X_{m a}\right.$ and $\left.P X_{s p}\right)$, divided by the background noise:

$$
C N R=\frac{P X_{s p}-P X_{m a}}{\sqrt{P X_{s p}}}
$$

A set of four images $\epsilon_{x x}=0.00,0.05,0.10$, and 0.15 was created for each of the CNR cases shown in Table 8 with average speckle radii of 2.5, 3.5, and 4.5 pixels. Examples of speckle images created with low and high contrast, shown in Fig. 64(a) and 64(c), and with added noise corresponding to CNR values of 4.0 and 12.9 in Fig. 64(b) and 64(d).

The images shown in Fig. 64(a)-64(d) illustrate the impact of noise on image quality. Fig. 64(b), having low contrast to begin with has poor image clarity after the additions of 


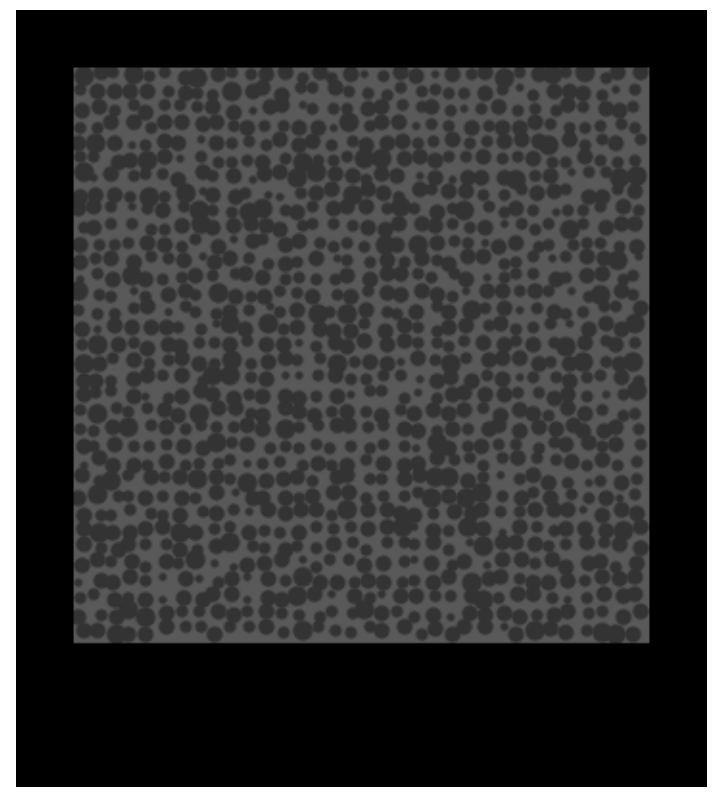

(a)

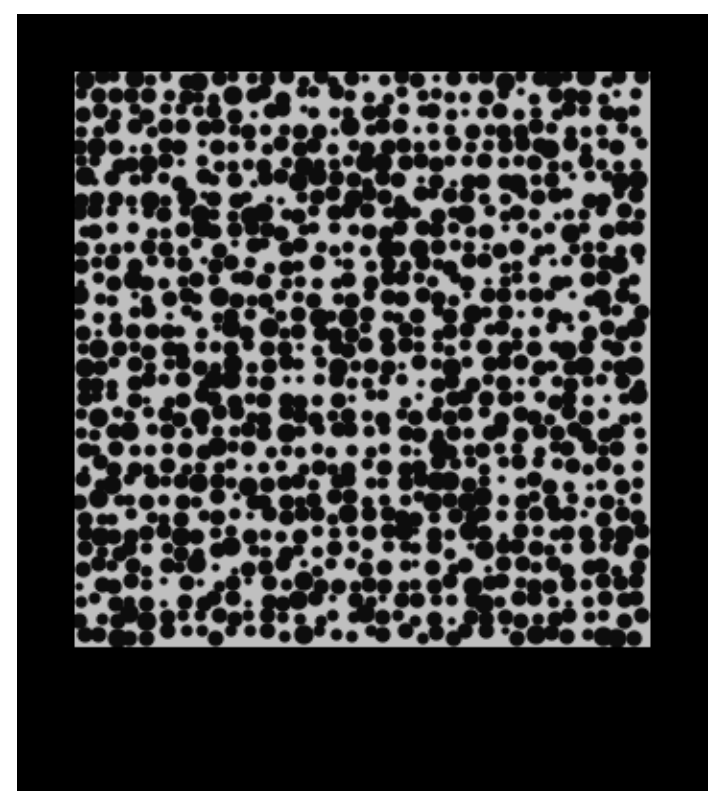

(c)

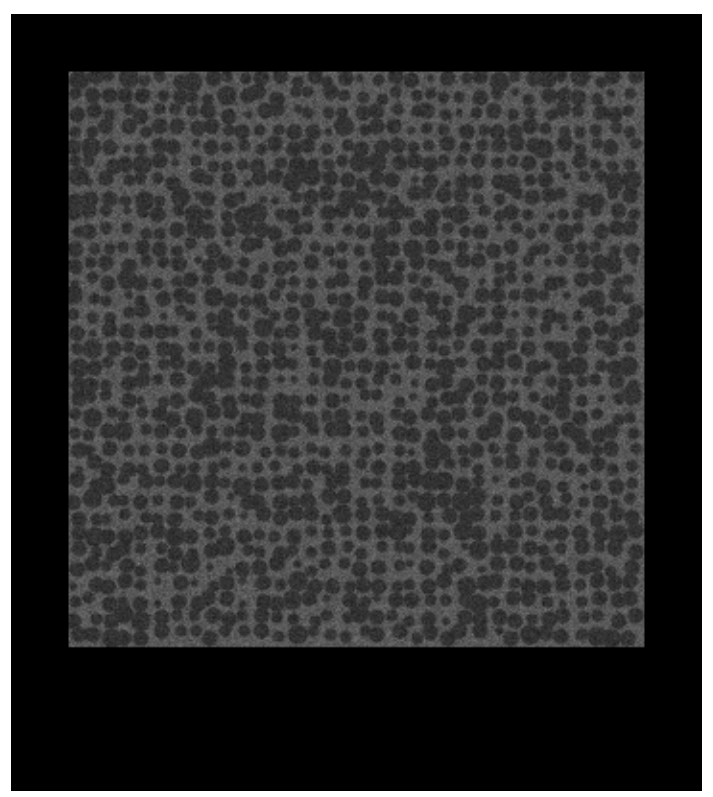

(b)

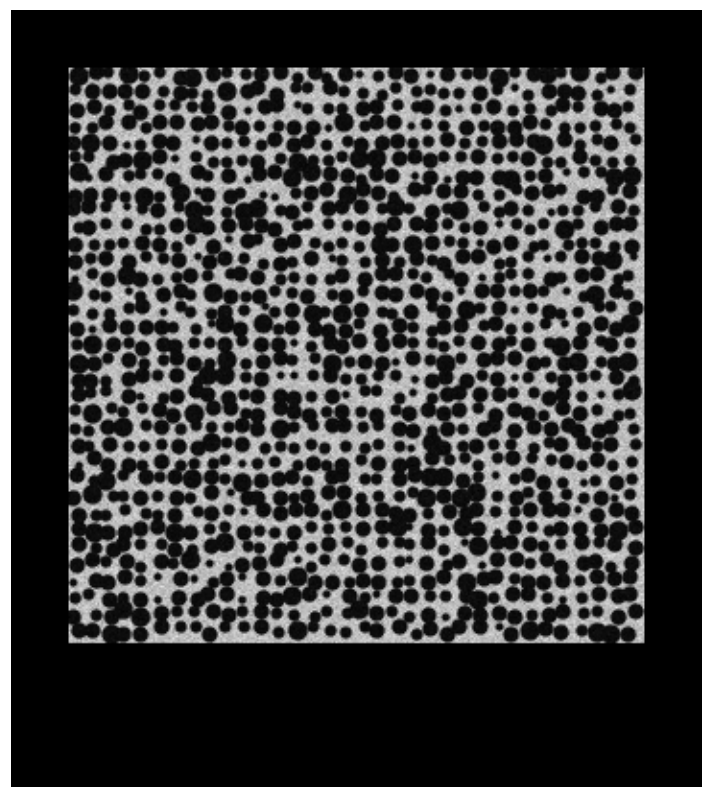

(d)

Figure 64: Examples of images used in DIC CNR error quantification analysis with average speckle radius $=3.5$ pixels: (a) Low contrast image; (b) Low contrast image with added noise $(\mathrm{CNR}=4.0)$; (c) High contrast image; and, (d) High contrast image with added noise $(\mathrm{CNR}=12.9)$. 
Table 9: Parameters used in DIC analysis of artificially produced images

\begin{tabular}{|c|c|c|c|}
\hline Image Type & \multicolumn{3}{|c|}{ Artificially produced } \\
\hline Image Noise & \multicolumn{3}{|c|}{ Artificial; Poisson noise } \\
\hline Reference ROI dims (pixels) & \multicolumn{3}{|c|}{$300 \times 300$} \\
\hline Applied Strain & \multicolumn{3}{|c|}{$5 \%, 10 \%$ and $15 \%$ with $v=1 / 3$} \\
\hline Speckle Radius (pixels) & $2.5 \pm 1.0$ & $3.5 \pm 1.4$ & $4.5 \pm 1.8$ \\
\hline Speckle Spacing (pixels) & $\sqrt{2 \pi} \pi^{*} 2.5 \pm 1.0$ & $\sqrt{2 \pi^{*}} 3.5 \pm 1.4$ & $\sqrt{2 \pi} * 4.5 \pm 1.8$ \\
\hline Subset Radius (pixels) & 14 & 20 & 26 \\
\hline Strain Radius (pixels) & 4 & 5 & 6 \\
\hline Subset Spacing (pixels) & \multicolumn{3}{|c|}{0} \\
\hline Seeds & \multicolumn{3}{|c|}{1} \\
\hline DIC Software & \multicolumn{3}{|c|}{ Ncorr [73] } \\
\hline Interpolation Method & \multicolumn{3}{|c|}{ Biquintic B splines } \\
\hline
\end{tabular}

shot noise. The noise present in Fig. 64(d) is less obvious due to the high contrast originally present.

Each set of images was analyzed in Ncorr for DIC using the parameters in Table 9. The speckles were designed to have three possible normally distributed radii: $2.5,3.5$, and 4.5, each with a standard deviation of $20 \%$ to better represent natural speckle variation and facilitate subset recognition from increased uniqueness. Speckle distribution was originally based on a square grid with spacing of $\sqrt{2 \pi}$ multiplied by the speckle radius to ensure an average $50 \%$ area coverage. The exact locations of speckles were varied normally to increase randomness while maintaining a suitable average speckle density.

\section{CNR Error Function: Results}

DIC analysis produced normal and shear strain values for every pixel at each deformed state $(5 \%, 10 \%$ and $15 \%$ strain). Two examples of the computed axial and lateral strain fields are shown in Fig. 65(a)-65(d). 


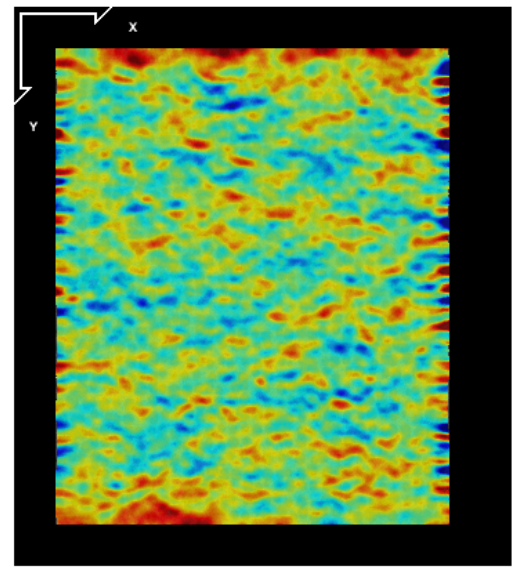

(a)

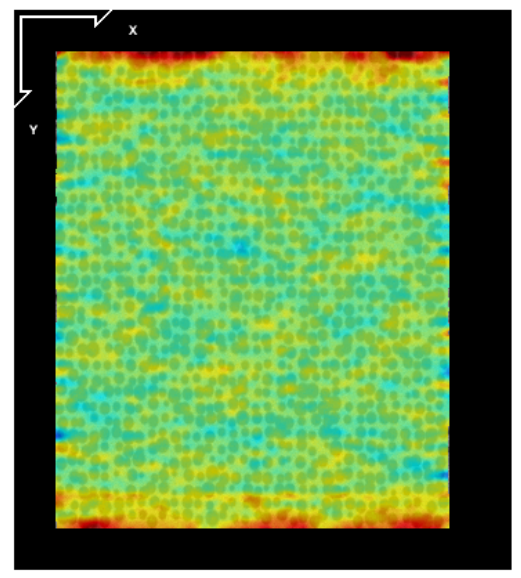

(c)
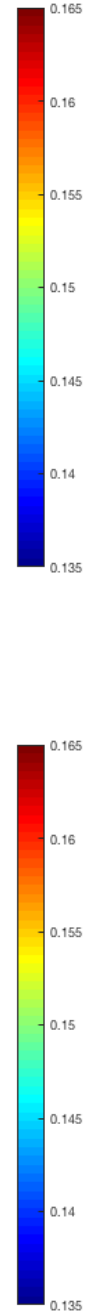
(b) $\epsilon_{x x}$ for $\mathrm{CNR}=4.8$; and, (c) $\epsilon_{y y}$ and (d) $\epsilon_{x x}$ for $\mathrm{CNR}=10.2$

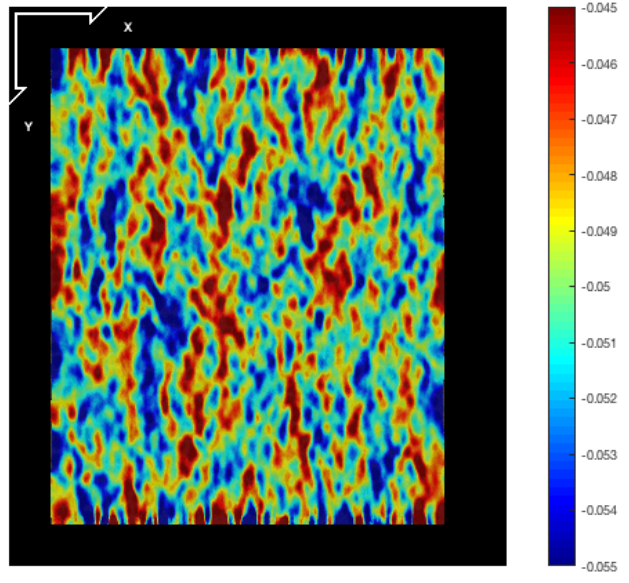

(b)

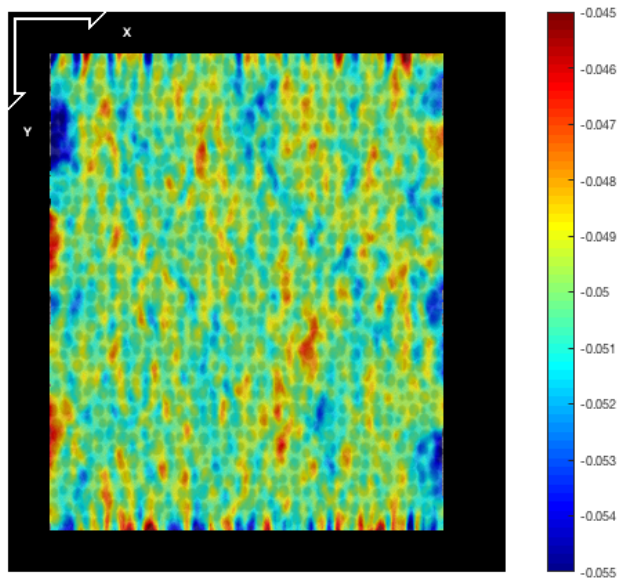

(d)

Figure 65: Sample normal strain fields computed for artificial images: (a) $\epsilon_{y y}$ and 
The axial strain fields computed by Ncorr were compared to the artificially applied strain to determine expected errors as a function of CNR. The 62,500 selected pixels were sufficiently far from the edge to avoid interpolation error effects. The residual error quantified here is the standard deviation of the difference between computed and applied strain for the analyzed pixels. First, the effect of applied strain level on residual error was an-

alyzed as a function of total speckle CNR, with the results shown in Fig. 66(b). Then, a study of the residual error for three speckle sizes at the same strain was performed, with the results shown in Fig. 66(a).

\section{CNR Error Function: Discussion}

The two sets of strain fields in Fig. 65(a)-65(d). show axial strain of 15\% and corresponding lateral contraction of $5 \%$. The colourbars show a range of $\pm 10 \%$ of the expected value (i.e., $13.5 \%-16.5 \%$ for $\epsilon_{y y}$ ). Neglecting high strains caused by interpolation errors around the edges, it is clear that in Fig. 65(a) and 65(b), where the original CNR was 4.8 , there are significantly more extreme values further from the theoretical value than in Fig. 65(c) and 65(d), where the CNR was 10.2. Due to the observed difference in DIC accuracy with varying pixel-level CNR, further discussion is required to quantify the uncertainty relationship. The CNR values discussed above correspond to single-pixel features and are scaled as per Eq. 66 to represent the total image-level CNR.

The effect of speckle size (radius of 2.5, 3.5, and 4.5 pixels $\pm 20 \%$ ) was examined and reported in Fig. 66(a). DIC parameters (Tab. 9) used in the analyses were approximately scaled with pixel radius. For example, the subset radius of 20 at a pixel radius of 3.5 should correspond to a subset radius of 14.28 and 25.71 for 2.5 and 4.5 pixel speckle radii, respectively. As integer values are required, exact parameter scaling was not possible. The first conclusion drawn from Fig. 66(a), is the absolute performace of DIC strain measurement with different size speckles was predictable when described by the image level CNR. Therefore, for accurate error prediction, speckle size should be considered in CNR calculation. 


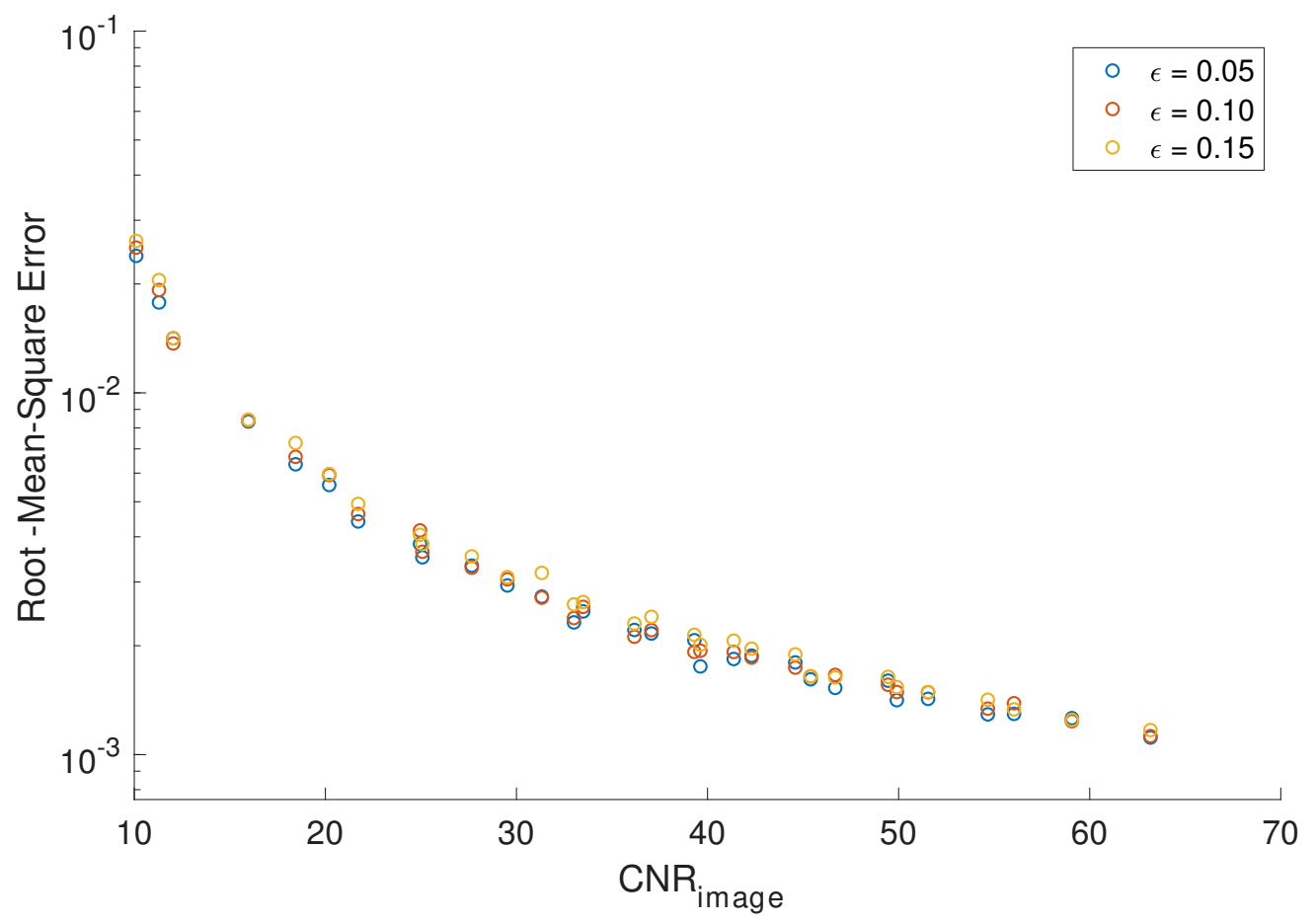

(a)

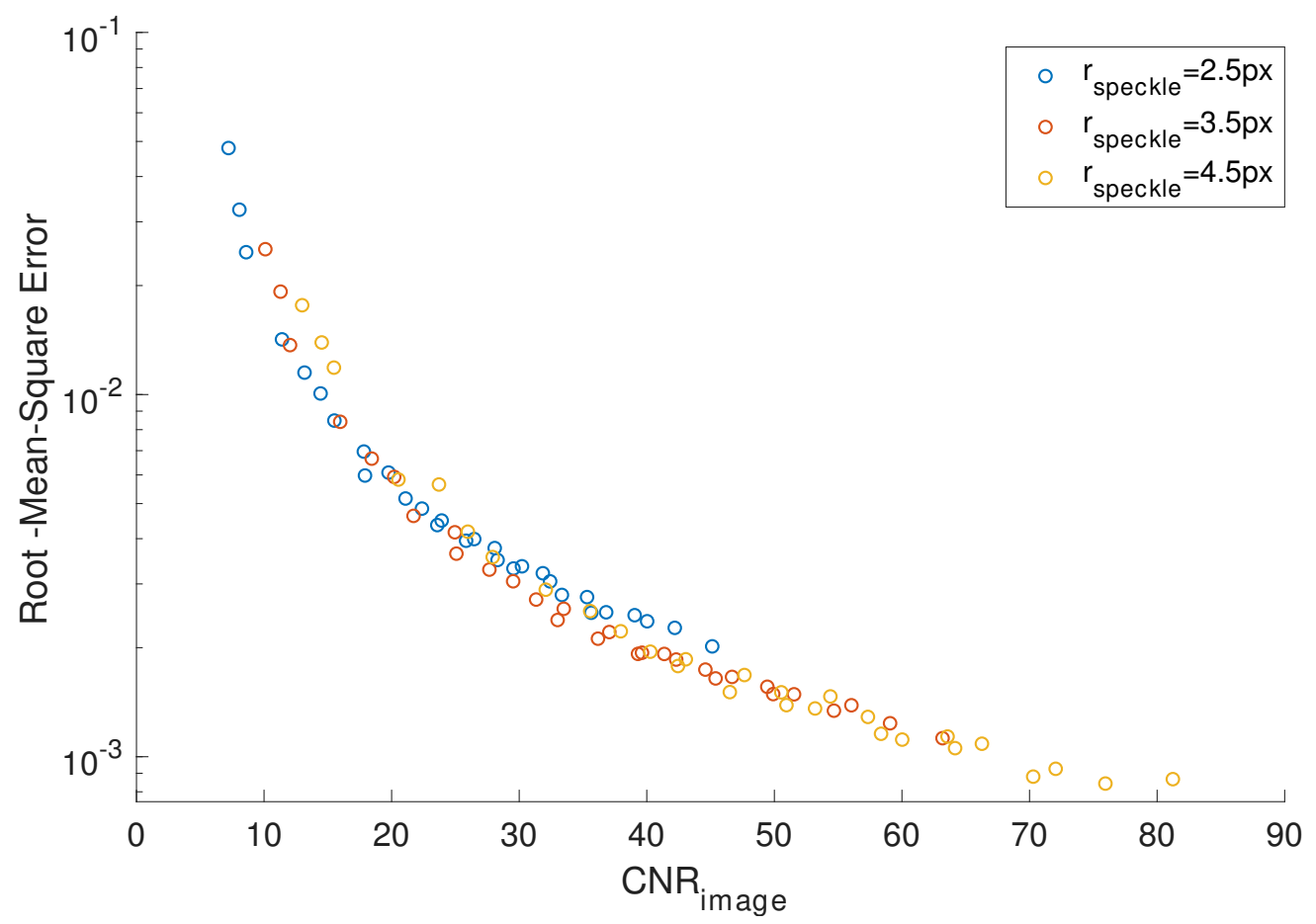

(b)

Figure 66: Relative standard error of residual pixel value (computed-theoretical) versus total contrast-to-noise ratio for (a) speckles of radius 3.5 pixels under applied strain $\epsilon_{y y}=0.05,0.1$, and 0.15 , and (b) speckles of radii $2.5,3.5$, and 4.5 pixels under applied strain $\epsilon_{y y}=0.15$. 
It is also observed from Fig. 66(a) that errors greater than 0.01 are expected when $C N R_{i}<20$. There is a diminishing return on increasing CNR to reduce computational errors. Beyond $C N R_{i}=40-50$, where the error is approximately 0.0011 , there is reduced benefit in increasing CNR. It is therefore recommended to design contrast markers to have a $C N R_{i}$ in the range of $40-50$.

As the standard deviation of pixelwise residual (actual strain - expected strain) was found to best represent trends in results, both in Fig. 66(a) and Fig. 66(b), it is suggested that the error caused by added Poisson noise is an absolute offset. This offset can be throught of as a \% pixel error in subset matching caused by finite errors in correlation coefficient minimization. The \% pixel matching error therefore increases with noise. As a result, and as supported by Fig. 66(b), higher strains will have a smaller relative uncertainty. This analysis was performed using Ncorr [73] DIC, therefore the effects on error caused by different interpolation functions was not evaluated.

Despite not being completely documented, increasing CNR did not only reduce residual measurement error, but also noticeably decreased computation time. The decrease in solution time is likely due to less iterations required to satisfy the Newton-Raphson algorithm residual requirements. As computation time may become significant for large studies at high frame rates (e.g., 100 analysis frames at $1000 \mathrm{fps}$ ), the compromise between intrusive markers and sufficient contrast may be further influenced by computation time. If convergence iterations are in fact the most important factor leading to increased computation time, the increase would be proportional to the number of subsets solved, therefore subset spacing could decrease computation time with only small changes in results when the CNR can not be increased by other means. The cause of the increased solution time is currently hypothesized to be attributed to increased Newton-Raphson convergence iterations; however, additional studies are required to quantify this effect.

As discussed in this section, image noise contributes to an absolute error in DIC strain measurement. The range of errors expected $\left(0.01\right.$ at $C N R_{i}=20$, and 0.001 at $\left.C N R_{i}=60\right)$, suggest that while noise does cause uncertainty, other sources of error such as out-of-plane motion may have more significant contributions. 
The error function relating an input image CNR to output error, as shown in Fig. 63, has now been quantified. As the error function outputs the same value for an identical input, the analytical model must output a similar result to those measured by the physical process. Therefore, the flowchart is further developed to include a calibration step (if needed). The calibration was performed by evaluating the physical process, the analytical model and the relationship between the two, as shown in Fig. 67.

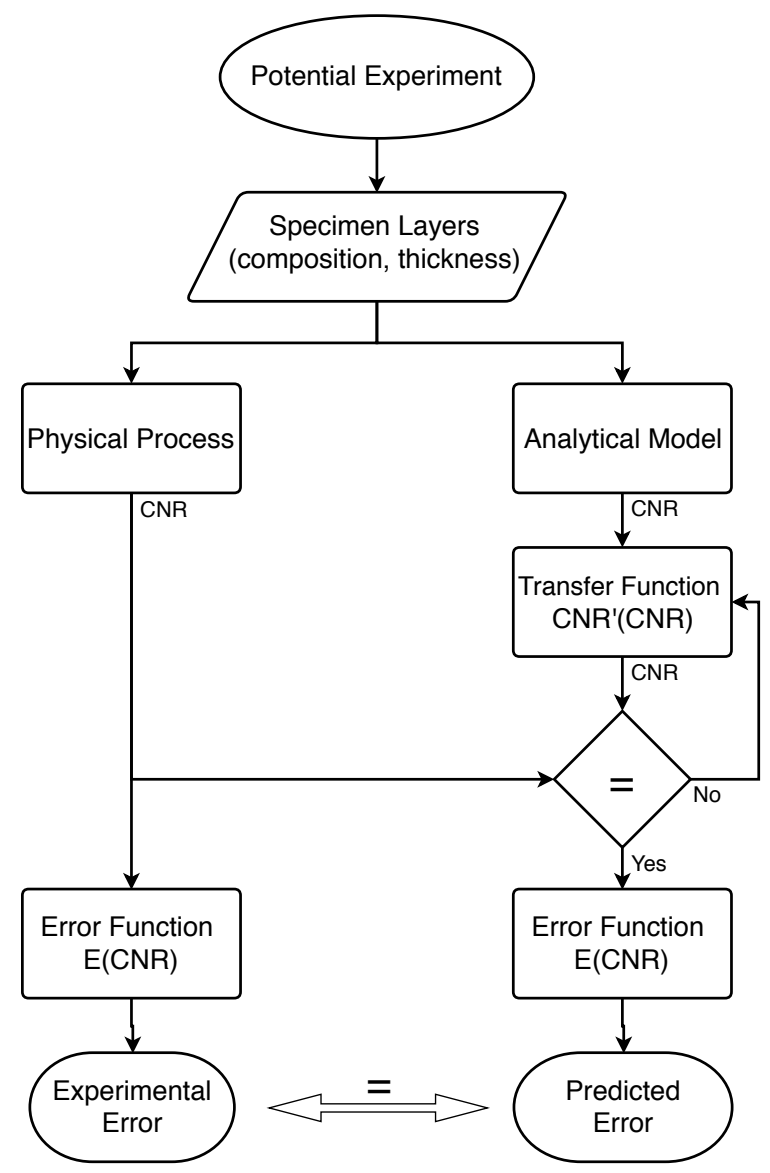

Figure 67: Flowchart demonstrating the steps required for analytical model calibration.

\subsubsection{Experimental DIC Error}

As a continuation of the artificial image DIC error analysis in the previous section, experimental data was collected to quantify measurement errors of the system caused by image 
Table 10: Measured pixel intensity and noise and corresponding CNR estimates at several power levels.

\begin{tabular}{cc|cc|cc} 
& \multirow{2}{*}{$\mathrm{k}$} & \multicolumn{2}{|c}{$I_{p x}$} & $\sigma_{p x}^{2}$ & $C N R_{p x}$ \\
$\mathrm{kV}$ & $\mathrm{mA}$ & Spec. & Mark. & & \\
\hline 70 & 100 & 22.7 & 17.9 & 0.17 & 11.9 \\
80 & 125 & 31.7 & 23.2 & 0.37 & 15.2 \\
90 & 160 & 46.4 & 32.3 & 0.57 & 18.7 \\
100 & 200 & 67.2 & 45.6 & 1.14 & 20.2 \\
110 & 260 & 97.1 & 65.1 & 2.27 & 21.2 \\
120 & 320 & 141.4 & 94.4 & 4.76 & 21.6
\end{tabular}

noise.

\section{Experimental DIC Error: Method}

The out-of-plane translation experiments (Sec. 6.1.1) with out of plane motion causing perceived strains of $3.4 \%, 7.0 \%, 10.8 \%$, and $15.0 \%$ were imaged at the power levels shown in Tab. 10. Data at $19.7 \%$ perceived strain was not included in this analysis due to variations between the computed and predicted results in Sec. 6.1.3, therefore error could not be isolated to contributions from noise. The $34 \mathrm{~mm}$ Humimic specimen containing a $3 \mathrm{~mm}$ contrast sheet composed of $50 \mathrm{wt} \% \mathrm{BaSO}_{4}-50 \mathrm{wt} \%$ Humimic results in different contrastto-noise ratios at each power level due to spectral attenuation variations. The pixel-level contrast-to-noise ratio, $C N R_{p x}$, was determined by measuring both the average value and variance in a 100x100 pixel subset of uniform attenuation (repeated for contrast layer and bulk medium). The generator current and voltage and corresponding measured average pixel intensity, $I_{p x}$, variance, $\sigma_{p x}^{2}$, and $C N R_{p x}$ are all shown in Tab. 10.

The reference images for each analysis are shown in Fig. 68(a)-68(f). DIC was performed on each of the sets of images corresponding to a generator voltage and current setting. A 100x100 pixel subset was used to determine the RMS error for each CNR. Otherwise, the DIC parameters used in this analysis are the same as in Tab. 7. The subset was 
Table 11: Measured CNR and RMS residual error at several input power levels.

\begin{tabular}{cc|c|cccc} 
& & & \multicolumn{5}{|c}{ RMS $_{e}$} \\
$\mathrm{kV}$ & $\mathrm{mA}$ & $C N R_{p x}$ & $\epsilon=3.4 \%$ & $\epsilon=7.0 \%$ & $\epsilon=10.8 \%$ & $\epsilon=15.0 \%$ \\
\hline 70 & 100 & 11.9 & 0.0043 & 0.0041 & 0.0047 & 0.0045 \\
80 & 125 & 15.2 & 0.0023 & 0.0028 & 0.0027 & 0.0030 \\
90 & 160 & 18.7 & 0.0016 & 0.0019 & 0.0020 & 0.0023 \\
100 & 200 & 20.2 & 0.0015 & 0.0016 & 0.0018 & 0.0021 \\
110 & 260 & 21.2 & 0.0011 & 0.0011 & 0.0013 & 0.0017 \\
120 & 320 & 21.6 & 0.0008 & 0.0010 & 0.0013 & 0.0017
\end{tabular}

centred in the X-ray exposure to reduce edge effects seen in out-of plane translations in Sec. 6.1.3, isolating the error to noise contribution.

\section{Experimental DIC Error: Results}

The measured RMS error is defined here as the root mean squared value of the predicted strain subtracted from the measured strain. It was computed for all cases shown in Tab. 10. The results for each applied strain level are shown numerically in Tab. 11 and graphically in Fig. 69.

As a comparison to results in Sec.6.2, the RMSe was plotted as a function of the CNR for both artificial and experimental images. The results are shown in Fig. 70 for $C N R_{p x}$ and Fig. $71 C N R_{i}$.

\section{Experimental DIC Error: Discussion}

It was originally hypothesized in Sec. 6.2 that DIC accuracy for a speckled image with random Poisson distributed noise would be comparable to images acquired by our system. It is clear from Fig. 70 that this hypothesis is not valid. Upon further examination, these artificially created images do not accurately represent the noise in X-ray images acquired 

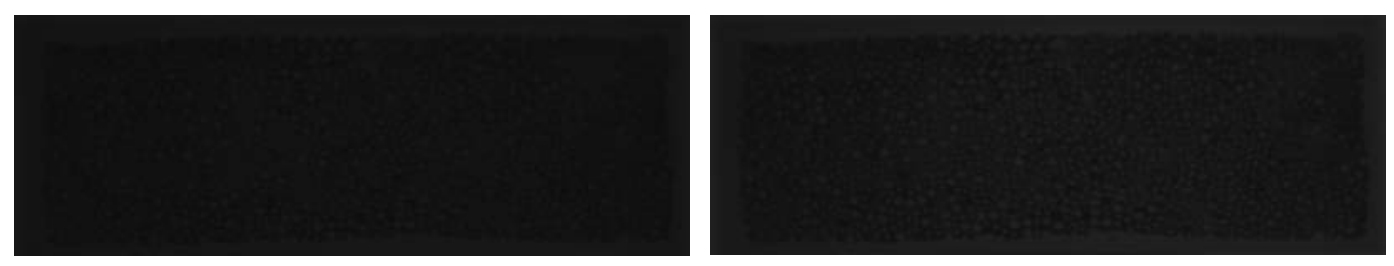

(a)

(b)
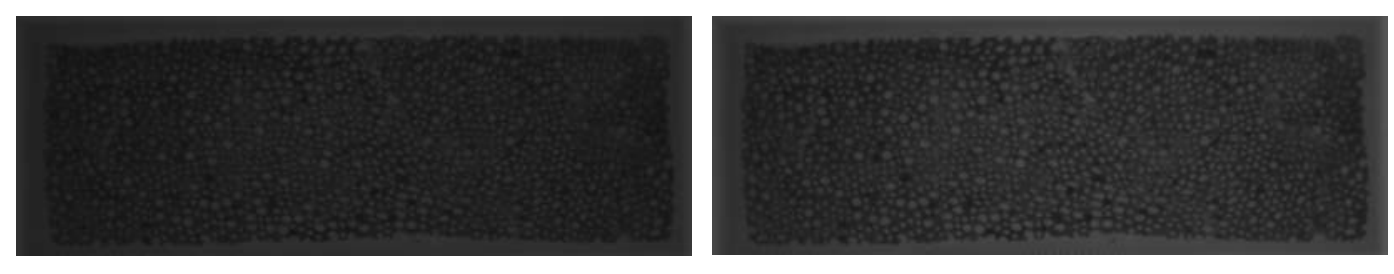

(c)

(d)
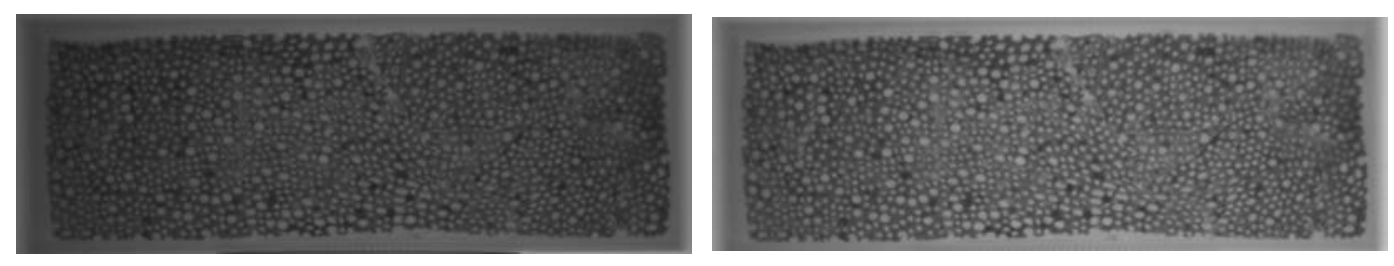

(e)

(f)

Figure 68: Reference images for experimental error analysis at (a) $70 \mathrm{kV}$ and $100 \mathrm{~mA}$, (b) $80 \mathrm{kV}$ and $125 \mathrm{~mA}$, (c) $90 \mathrm{kV}$ and $160 \mathrm{~mA}$, (d) $100 \mathrm{kV}$ and $200 \mathrm{~mA}$, (e) $110 \mathrm{kV}$ and $250 \mathrm{~mA}$, and (f) $120 \mathrm{kV}$ and $320 \mathrm{~mA}$. 


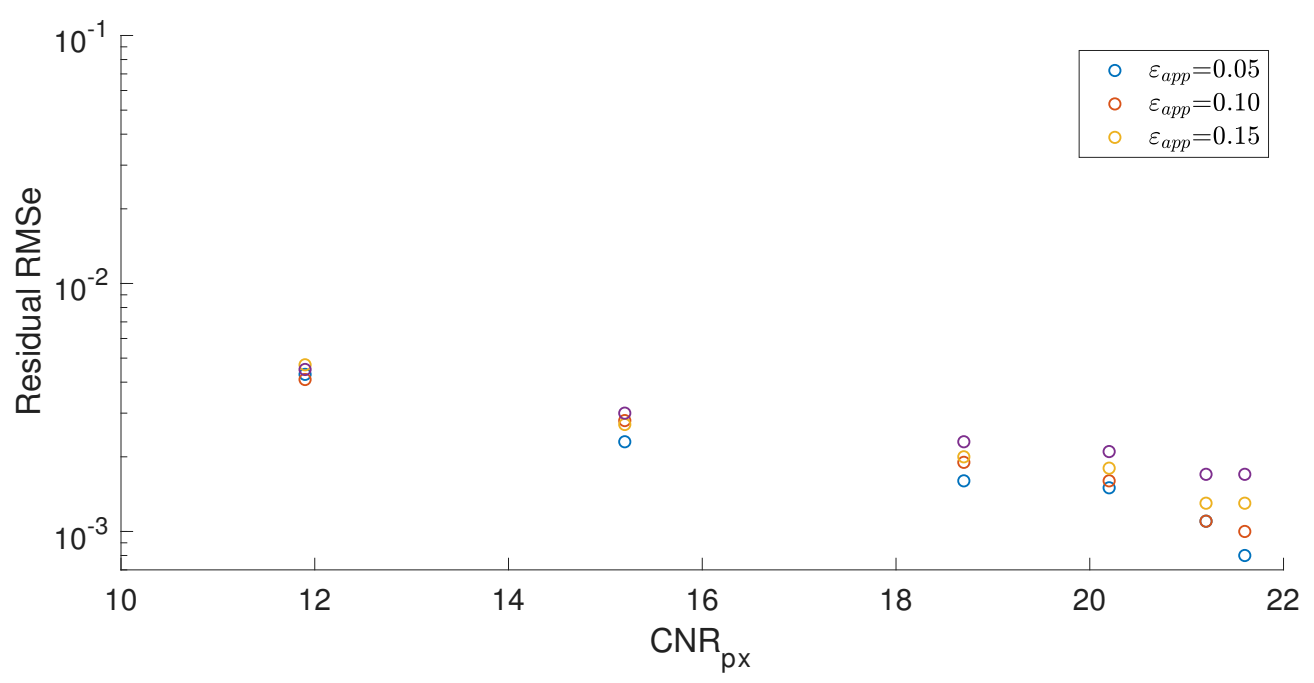

Figure 69: Measured residual RMSe of DIC analyses versus pixel-level contrast-tonoise ratio at varying power levels.

using our system. Although the noise basis is originally Poissonian, this distribution applies to the number of photons reaching the sensor at each energy level. Therefore, the sum of variances from spectral energy-weighted photons, and cascading secondary quantum processes change the noise characteristic. As such, the results of Sec. 6.2 correspond to an idealized lower bound on DIC error for a monoenergetic beam. Therefore, it does not accurately represent the physical processes of image acquisition. The range of anticipated errors are found to be similar; however, the range of pixel-level CNRs is quite different for the two. It could be suggested that the four experimental points having the lowest CNR values follow a trend resembling the results from the artificial images. There is a notable step down in error before the two final points. Although the exact cause is not known, either a smaller than expected difference in signal, or a larger variance for these two data points could be responsible. It is hypothesized that a relative increase in X-ray transmission (i.e., lower attenuation) at increased energy decreases the effective contrast between the two layers. Therefore suggesting that it is a less significant increase in signal difference than anticipated. Alternatively, an increased in signal variance from a larger quantity of high energy photons being absorbed by the material may be the cause, as per Eq. 55 .

An interesting result from Fig. 71 and 68(a)-68(f), is the range of errors expected due to 


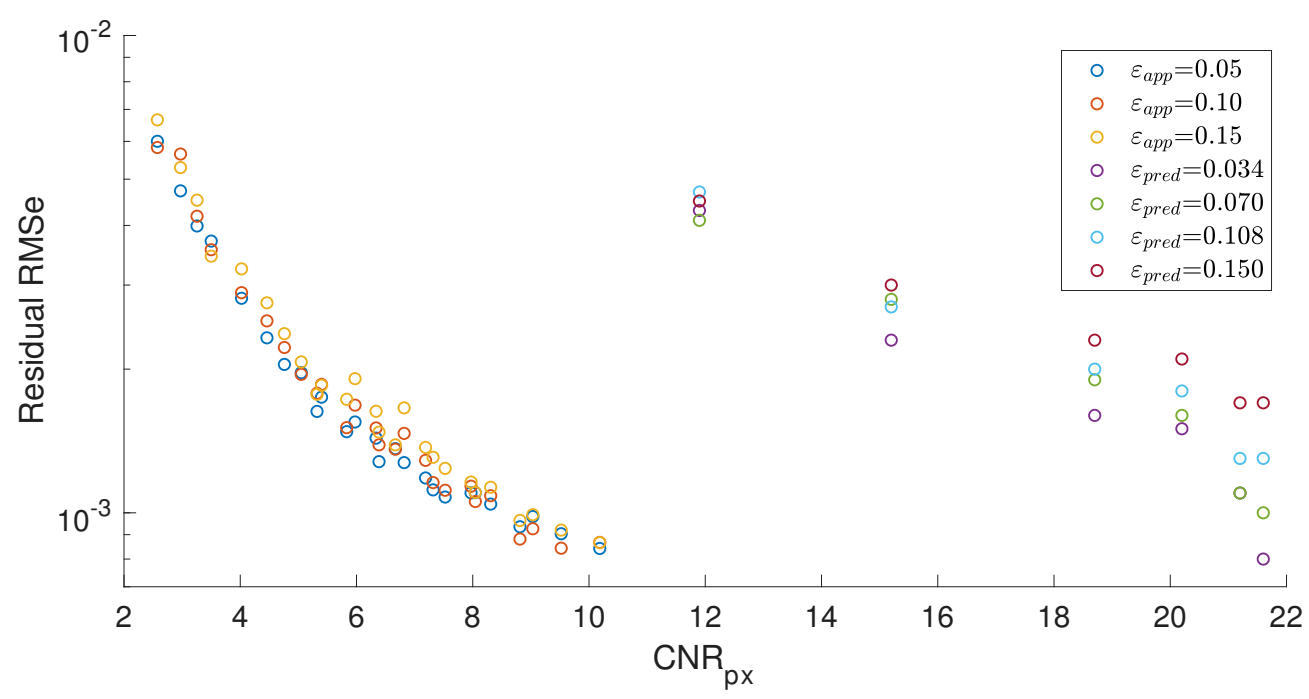

Figure 70: Root mean squared error of residual (predicted-measured) as a function of pixel-level CNR for strain applied to artificially generated images $(r=4.5$ pixels) and experimentally produced images ( $r \geq 5$ pixels).

contrast-to-noise ratio. Even in images with very low luminosity (Fig. 68(a)), the variance is sufficiently low such that the RMS error is below $10^{-2}$. It is recommended that the image-level CNR approach 200 to reduce errors below $10^{-3}$. In practice, this is achieved by either increasing doping concentration, contrast layer thickness, or marker size. A further conclusion from the previous two chapters is the relatively small contribution of expected error from CNR as compared to out-of-plane motion. It appears that reducing out-of-plane motion is therefore more critical to accurate DIC measurements than noise thresholds.

\subsubsection{Analytical Model Validation}

As described in Sec. 4.1, the analytical model requires specific input values including generator current and voltage, dimensions, and specimen composition. After transfer function calibration, the analytical model should output a similar CNR to experimental measurements with identical parameters. Calibration was performed in two steps, first, the camera gain was approximated, then CNR calibration was performed. Both were based on the measured value from images used in previous analyses. 


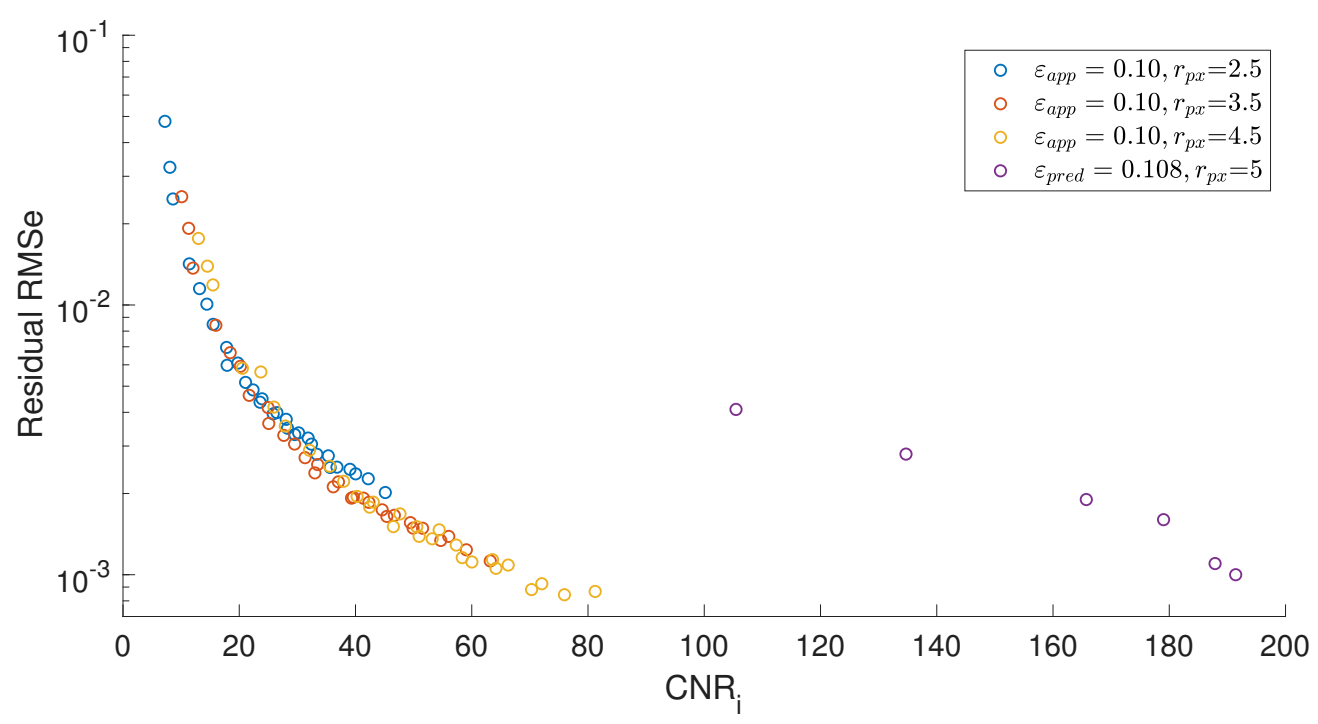

Figure 71: Root mean squared error of residual (predicted-measured) as a function of image-level CNR for strain $(\epsilon \approx 10)$ applied to artificially generated images $(\mathrm{r}=2.5,3.5$, and 4.5 pixels) and experimentally produced images ( $\mathrm{r} \geq 5$ pixels).

\section{Transfer Function: Calibration}

The first step required for model calibration is to approximate the camera gain. The remaining gains and parameters were determined using manufacturer or published literature and are generally well approximated. At this point, the analytical model output is at the electron level in the camera sensor. In order to translate this value to pixel intensity, the specimen and experimental parameters from Tab. 10 were used as inputs to the analytical model. The resulting estimates of electron count with measured pixel intensities are shown in Tab. 12 and Fig. 72.

The slope in Fig. 72 is used to approximate the camera gain. Therefore, an approximate relationship between analytical and measured values is established. After gain calibration, the contrast-to-noise ratio was compared for the experimental results and computational model. To determine the ability of the model to predict contrast-to-noise ratio between models. The CNR was determined between images at all energies (e.g., $70 \mathrm{kV}-100 \mathrm{ma}$ and $120 \mathrm{kV}-320 \mathrm{ma}$ ) as a controlled surrogate for two intensities in the same image. Values 
Table 12: Measured pixel intensity and noise and analytical approximations at several power levels.

\begin{tabular}{cc|cc|cc} 
& \multirow{2}{*}{$\mathrm{k}$} & \multicolumn{2}{|c}{$I_{p x}$} & \multicolumn{2}{c}{ electroncount } \\
$\mathrm{kV}$ & $\mathrm{mA}$ & Spec. & Mark. & Spec. & Mark. \\
\hline 70 & 100 & 22.7 & 17.9 & 136 & 55 \\
80 & 125 & 31.7 & 23.2 & 491 & 235 \\
90 & 160 & 46.4 & 32.3 & 819 & 414 \\
100 & 200 & 67.2 & 45.6 & 1301 & 686 \\
110 & 260 & 97.1 & 65.1 & 2040 & 1112 \\
120 & 320 & 141.4 & 94.4 & 3038 & 1701
\end{tabular}

were calculated for the analytical and experimental data and plotted in Fig. 73.

\section{Transfer Function: Discussion}

The camera gain calibration is shown to be successful in Fig. 72, as a linear relationship $(\mathrm{m}=0.072$ or $\mathrm{m}=0.062$ and $\mathrm{b}=17.816)$. In theory, a line passing through the origin would better-represent the ideal system; however, due to readout noise in the camera (i.e., dynamic range floor), a minimum pixel value will always be produced, even in complete darkness. Therefore, the affine transformation should be used to represent the physical process, and can be used reliably.

After calibration of camera gain, CNR was compared between experimental analyses and the physical experiment (Fig. 73). The process is non-linear, and displays characteristics of $y=x^{1 / p}$, with $\mathrm{p}>1$. A linear best-fit curve for the first four data points was plotted as an approximate relationship for low CNR values; however, it is not a sound indicator of the relationship between the two contrast-to-noise ratios. The analytical model is able to predict pixel intensity, yet is limited in its ability to predict CNR. Therefore, it can be concluded that limitations in the model are caused either by incorrect approximation of the noise variance, or by approaching sensitivity limits on the camera. That is, the analytical model does not account for the full well capacity (i.e., maximum number of electrons 


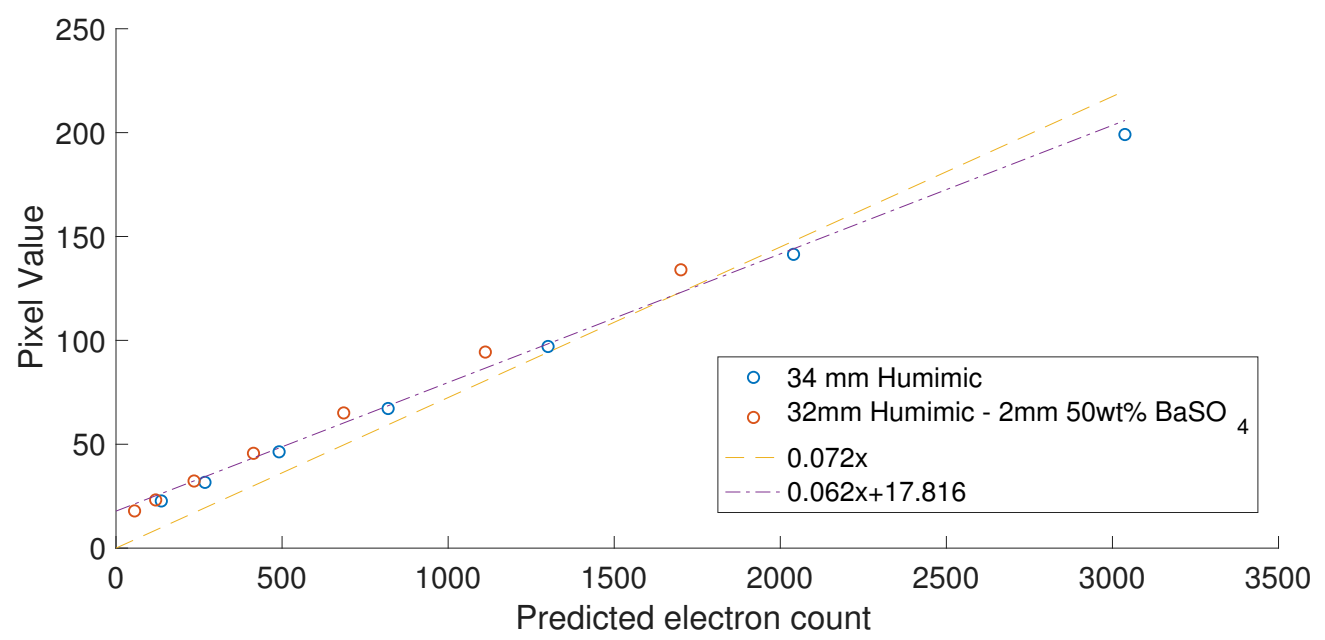

Figure 72: Pixel intensity as a function of electron count estimated by the analytical model camera gain calibration.

per pixel), leading to errors near saturation. As our high-speed applications are generally far from saturation, only the lower 3-4 data points in Fig. 73 are meaningful. One specific assumption that may not have been valid was the approximation that for our system, the modulation transfer function (MTF) in Eq. 60 was approximately equal to one as only the zero-frequency term was considered. The validity of this assumption was tested by approximating the MTF via the contrast transfer function (CTF). A Leeds Test Object, with resolution of 0.6-2.2 line-pairs/ $\mathrm{mm}$ was used to evaluate the CTF for a range of input energies. The results are shown in Fig. 74.

Unfortunately, the approximated MTF shown in Fig. 74 invalidates the simplifying assumption used in Eq. 60. The previous justification was the following: if the marker size is sufficiently large, and the MTF is concave down towards decreasing resolution (i.e., approaching $100 \%$ ), then a zero-frequency approximation is valid. As the MTF curve is concave up, it is unclear where the function will tend towards $100 \%$, meaning markers significantly larger than $1 \mathrm{~mm}$ may be required for the method used to be valid. Alternatively, the following frequency domain integral must be determined for correct variance 


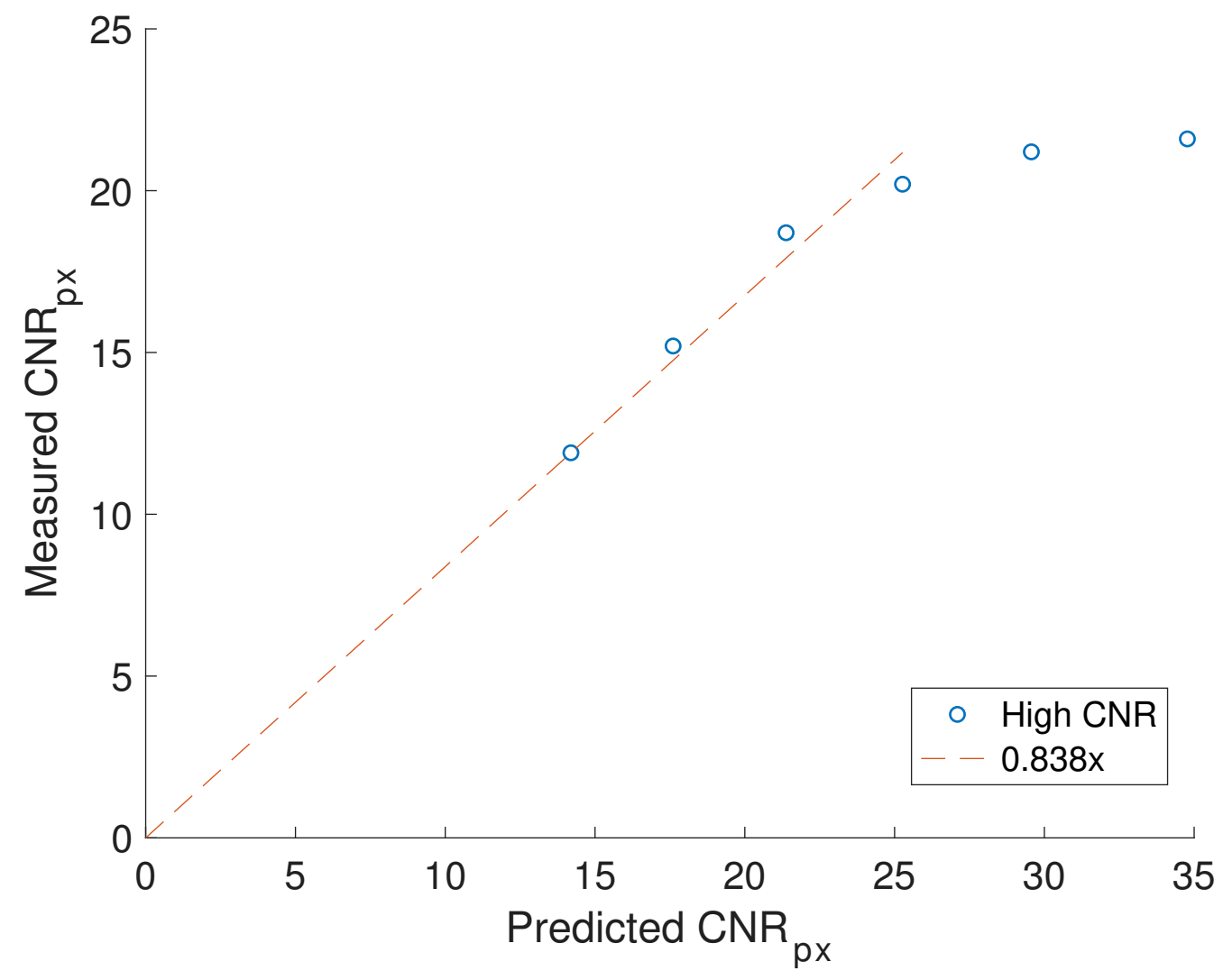

Figure 73: Measured versus actual $C N R_{p x}$ for a range of energy levels.

determination [26].

$$
\int_{-\infty}^{\infty} \int_{-\infty}^{\infty} \operatorname{MTF}^{2}(u, v) d u d v
$$

Another area where the variance could have been poorly approximated is in the final step of camera gain. Readout noise and dark current both contribute to final quantum noise power spectrum amplification, but were not quantified or included in the analysis. Despite severe limitations in the CNR approximation, it can be used to determine the order of magnitude of anticipated experimental results and assist in marker design, but cannot be used as a direct tool to determine the CNR of the physical process. 


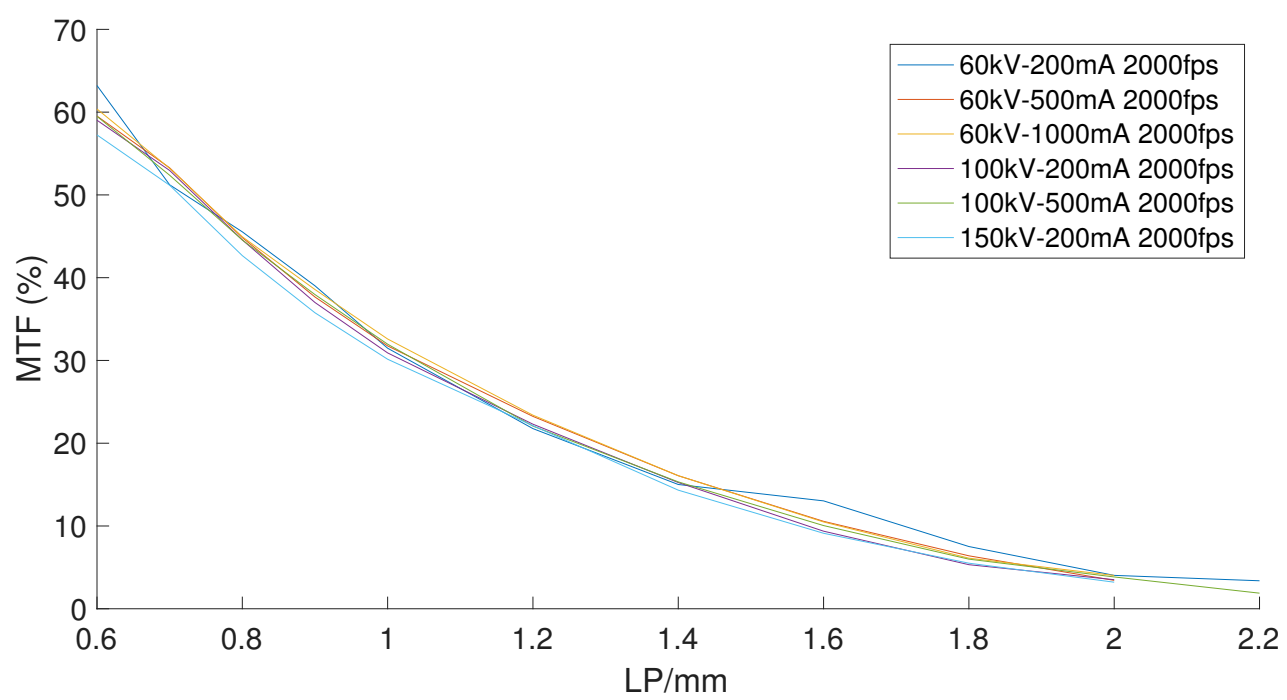

Figure 74: Modulation Transfer Function for a range of powers at 2000 frames per second.

\subsection{Preliminary Headform Study}

To demonstrate a possible application of the method discussed in this paper, a preliminary headform study was conducted. A drop test, using a NOCSAE compliant twin-wire carriage [74], was performed from a height of $55 \mathrm{~cm}$, and imaged at $1000 \mathrm{fps}$. The $\mathrm{BI}^{2} \mathrm{PED}$ headform used in this analysis, shown previously in Fig. 51, was provided by our partners at DRDC Valcartier.

Marker integration was first performed by partially sectioning the Sylgard 527 brain $\left(\rho=0.97 \mathrm{~g} / \mathrm{cm}^{3}\right)$ in a parasagittal plane $25 \mathrm{~mm}$ from the central plane (Fig. 75$)$. Pre-cast contrast markers composed of a $40 \mathrm{wt} \% \mathrm{BaSO}_{4}-60 \mathrm{wt} \%$ Sylgard 184 mixture $\left(\rho=* \mathrm{~g} / \mathrm{cm}^{3}\right)$ were randomly distributed across the section. The brain was re-fused using Sylgard 527 and placed in the headform. The headform was perfused with water to simulate cerebrospinal fluid, making the brain neutrally buoyant in the skull cavity. 


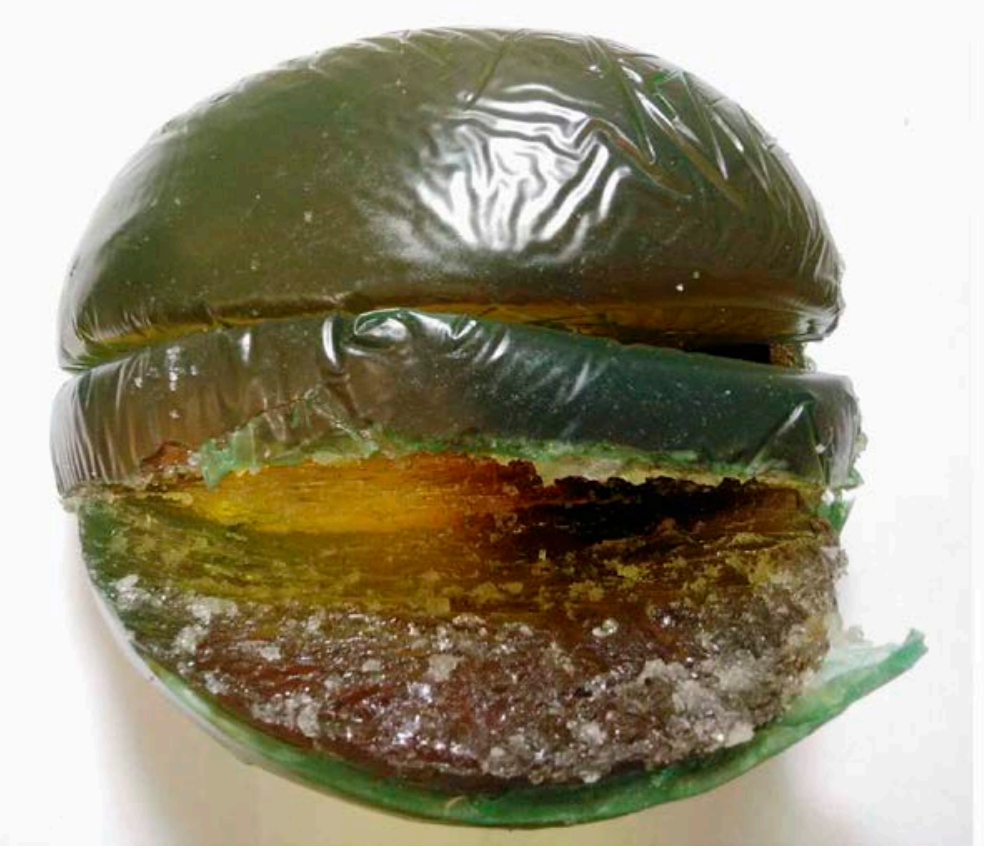

Figure 75: Top view of $\mathrm{BI}^{2} \mathrm{PED}$ brain sectioned for marker integration

\subsubsection{Results}

An X-ray image of the headform with an exposure time of $<1 \mathrm{~ms}$ is shown in Fig. 76. This image also shows the hardware mounting the $\mathrm{BI}^{2} \mathrm{PED}$ headform to the NOCSAE drop carriage [74], [72].

Trial image correlation was performed on a series of images from the drop test shown in Fig. 76. The purpose of this study was to validate the technique for displacement measurement inside a headform. Select quiver plots, from $10 \mathrm{~ms}$ to $25 \mathrm{~ms}$ post-impact, built using Ncorr DIC displacement data are shown in Fig. 77. The quiver plots for $5 \mathrm{~ms}$ to $50 \mathrm{~ms}$ post-impact are shown in larger format in Appendix D. Large displacements have long, red arrows, and small displacements have short, blue arrows. The DIC parameters used in this analysis are shown in Tab. 13. 


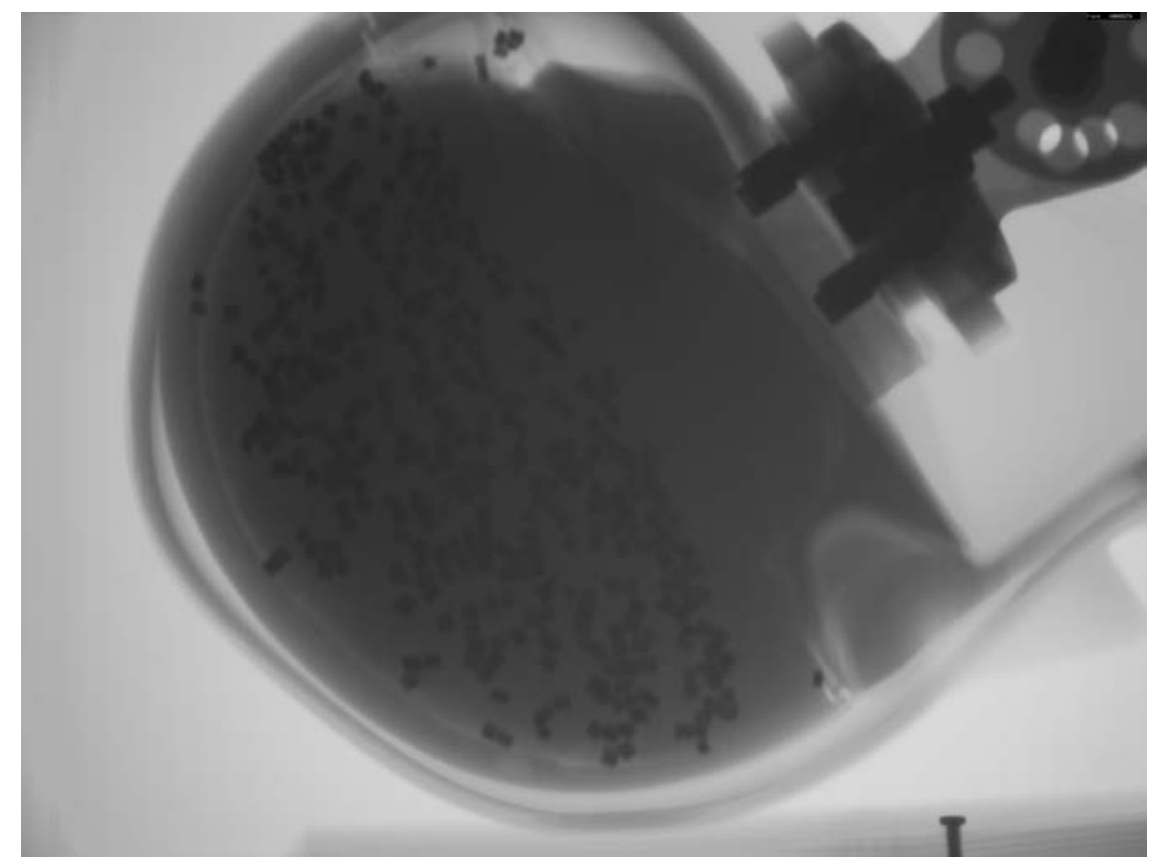

Figure 76: X-ray image of the BIPED headforms with individually placed markers integrated in a pre-cast brain. Dark regions represent integrated contrast markers.

Table 13: Parameters used in DIC analysis of $\mathrm{BI}^{2} \mathrm{PED}$ headform.

Image Type
Lens
Reference ROI dims (pixels)
Applied Deformation
Speckle Radius (pixels)
Subset Radius (pixels)
Strain Radius (pixels)
Subset Spacing (pixels)
Seeds
DIC Software
Interpolation Method

Experimentally acquired

Nikkor f/1.2, 50mm

irregular: 1118x1394

$55 \mathrm{~cm}$ drop

10-15

50

15

5

1

Ncorr [73]

Biquintic B splines 


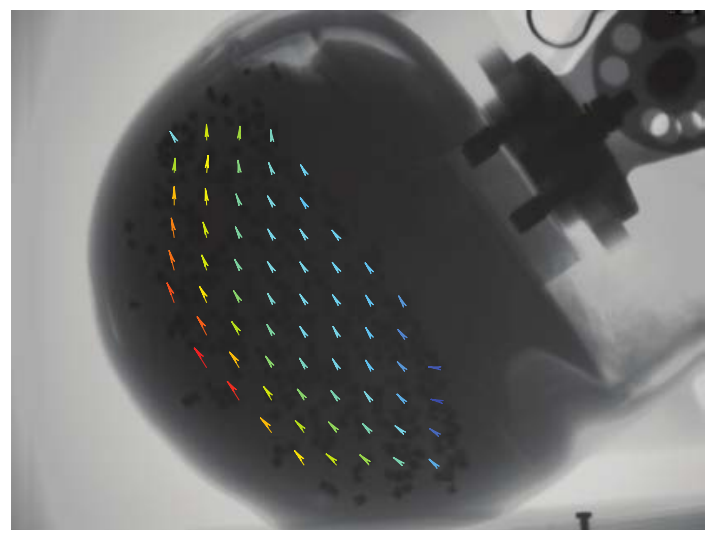

$10 \mathrm{~ms}$

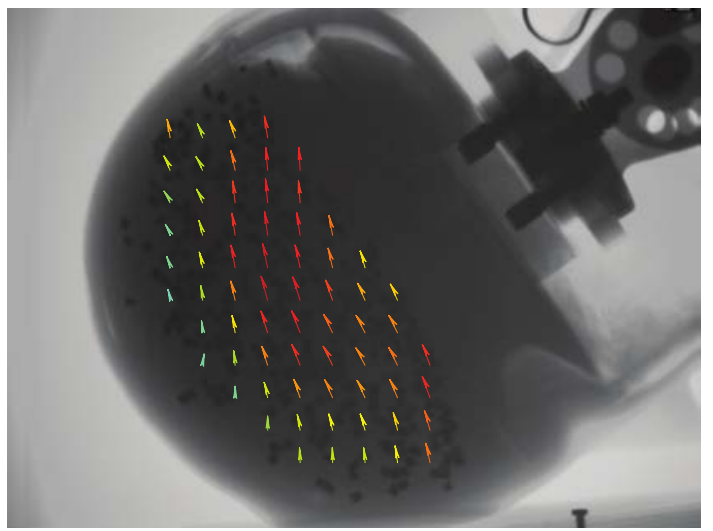

$20 \mathrm{~ms}$

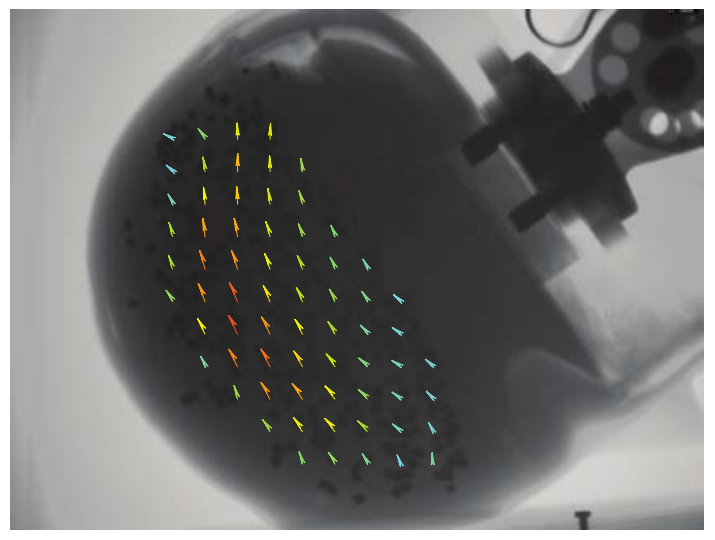

$15 \mathrm{~ms}$

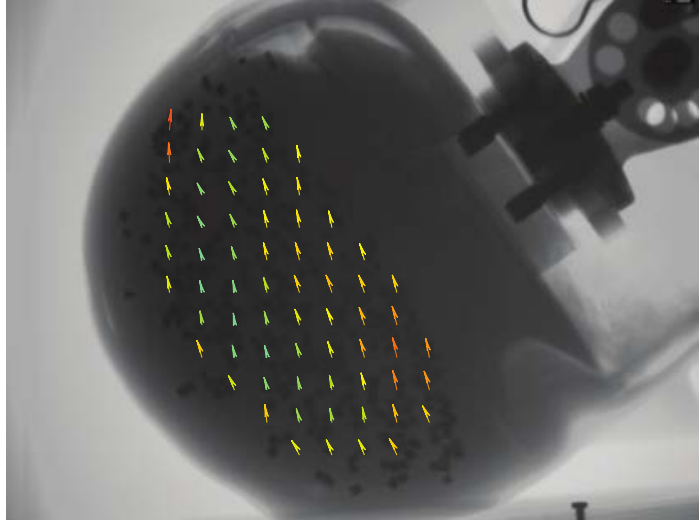

$25 \mathrm{~ms}$

Figure 77: Displacement quiver plot from DIC data for the $\mathrm{BI}^{2} \mathrm{PED}$ headform after a $55 \mathrm{~cm}$ drop captured at $1000 \mathrm{fps}$. 


\subsubsection{Discussion}

Despite being a preliminary method validation experiment, several interesting observations are possible. First, Ncorr [73] was reliably able to compute displacement and strain fields in the headform despite low contrast in the high-speed X-ray images. This suggests the method described in this work can be extended to upcoming cadaveric studies or for future helmet evaluation using biofidelic headforms.

Notably, in Fig. 77, there is propagation of a stress wave from left to right in the period of $10 \mathrm{~ms}$ to $25 \mathrm{~ms}$ post-impact. This is interesting as it supports work from [60] in cadaveric brain studies, where implanted markers movement followed distinct looping patterns. These looping patterns are consistent with the propagation of stress waves as shown in the $\mathrm{BI}^{2} \mathrm{PED}$ headform.

This experiment also suggests temporal resolution requirements for future experiments. Although not directly measured, the impact velocity was approximately 3.0$3.5 \mathrm{~m} / \mathrm{s}$, determined from the drop height and energy conservation. In this case, a temporal resolution of $5 \mathrm{~ms}$ would be insufficient to capture peak displacements and strains based on the stress wave propagation velocity (10-15 ms to travel the width of the view studied).

A previous attempt at marker integration involved the casting of a low melting temperature thermoplastic brain. This material demonstrated limitations in the technique as marker migration occurs when the bulk medium is prone to creep. As demonstrated earlier in this section, planarity is critical to proper displacement and strain characterization. Unfortunately, with marker creep, it is difficult to maintain planarity. Additionally, the markers may creep relative to the bulk material during dynamic impacts, further biasing the results as their displacement does not represent the motion of the specimen. Therefore, it is strongly recommended that either a high melting temperature thermoplastic that does not exhibit creep at ambient temperature, or, a thermosetting polymer be used as a tissue simulant forming the bulk medium. 


\section{Chapter 7}

\section{Conclusion}

This work describes the implementation of an uncoupled, high-speed cineradiographic facility for dynamic material characterization. The system was designed to study brain deformation in cadavers during impact events to generate material data critical to the development and validation of computational brain injury models. Increased understanding of biological tissue mechanical response, coupled with modern injury surrogate headforms will increase the understanding of impact induced brain injury, potentially improving future helmet design. The purpose of this thesis was to finalize the design of a high-speed X-ray system, to develop and validate a method of quantifying displacement and strain of optically inaccessible internal planes, to identify and quantify sources of error and measurement uncertainty, and provide technical recommendations on the future implementation of this method in upcoming cadaver studies. The following is a summary of the five research objectives described at the onset of this study, and the final comments and recommendations on each topic.

\section{High-speed X-ray lab implementation}

The high-speed X-ray lab is able to record dynamic impact events at rates exceeding $10^{4}$ frames per second. The current configuration allows for the study of impact-induced events using a free-flying linear impactor from 3 to $10 \mathrm{~m} / \mathrm{s}$. An $80 \mathrm{~kW}$ tungsten-rhenium rotating anode is used to produce a continuous X-ray photon flux with sufficient intensity 
to penetrate a head at up to $10,000 \mathrm{fps}$. As the system is used for mechanical characterization of materials, absorbed X-ray dose significantly higher than those used in medical diagnostic systems is possible. Therefore, commercially available X-ray detectors, designed for imaging living subjects do not achieve sufficient recording rates as this unnecessarily increases patients exposure and corresponding long term cancer risk without a significant increase in diagnostic information. Image acquisition is performed using an uncoupled optical system composed of a thallium-doped cesium iodide scintillator and a high-speed camera capable of recording at much higher rates than commercially available flat panel detectors. The system was designed to optimize light sensitivity by selecting a camera and scintillator with similar spectral responses. That is, the camera quantum efficiency curve is well-coupled to the spectrum produced by a CsI(Tl) scintillator.

\section{Contrast marker method development}

Homogeneous materials do not provide sufficient contrast for X-ray image correlation. Therefore, the design and implementation of radiographic contrast markers is an essential step in XDIC. Implanting markers having a different material composition inside a specimen produces speckles in images suitable for DIC. In most studies (i.e., cadaveric heads), the bulk specimen specimen and thickness are experimental parameters that cannot be changed, resulting in a fixed spectral attenuation profile. Generating sufficient X-ray photons to produce high-quality images through the specimen at high-speeds may not always be feasible. A method of designing suitable contrast markers was proposed. In literature, most applications used lead or tin shavings as a contrast medium; however, the importance of dynamic coupling of the markers with the bulk material is often ignored. Therefore, the method proposed designing markers that provide just enough contrast for accurate DIC analyses thereby maximizing dynamic coupling of the different materials.

Optical measurement methods achieve high imaging speeds by increasing ambient lighting. This is not possible in high-speed radiography with thick specimens due to inefficient X-ray photon generation. Only a fraction of energy in tube generators is converted to X-ray photons, while the remainder is lost as heat. An upper bound for energy incident 
on the anode to avoid damage from insufficient heat dissipation prevents increasing X-ray photon flux to increase image brightness. Therefore, as limited light reaches the sensor, shot noise from the finite number of photons reaching the sensor may become significant and affect measurement accuracy.

\section{Experimental validation}

Two methods were used to validate the contrast marker integration procedure described previously. First, a contrast sheet was placed in the mid-plane of a specimen during casting. The object was then translated or rotated out of plane. This method was found to provide sufficient contrast for measurement using DIC as the computed strains were sufficiently close to the predicted values. A second method, whereby a large quantity of similarly sized markers are distributed in the specimen after partial casting was performed in a biofidelic headform. The deformation studied after a drop test showed the propagation of stress waves inside the brain.

It was determined that a pre-made contrast sheet was a more effective and repeatable process for marker integration than randomly placing individual markers, where possible. It should be noted that the validation was performed on specimens cast partially before marker integration with the remainder subsequently cast after embedding. No attempts were made to surgically embed markers in a pre-made specimen or biological sample.

\section{Error quantification}

Two primary sources of error were identified and quantified in this work. First, shot noise caused by the finite number of X-ray photons reaching the sensor was studied. Both artificially created images and experimental results suggest that increased image noise, quantified herein as the contrast-to-noise ratio, decreases DIC accuracy. Although hypothesized to be an important consideration, quantification of errors caused by improper dynamic coupling between the bulk medium and contrast markers was beyond the scope of this work. Second, out-of-plane motion was analyzed as a potential source of error for $\mathrm{XDIC}$, as seen in its optical DIC counterpart. In radiography, out-of-plane motion results 
in an increased or decreased magnification of the object projection on the scintillator. This change in magnification results in a discrepancy between applied and perceived strains, influencing DIC accuracy.

It was found that out-of-plane motion is likely to be the dominant source of error. Although it may be impossible to avoid in certain dynamic studies, out-of-plane motion error should be minimized by placing the object as close as possible to the scintillator and far from the source. As perceived strain is dependent on the out-of-plane motion of a particular region, both the location of the centre of rotation and the corresponding magnitude of rotation will impact DIC accuracy.

\section{Technical recommendations for future studies}

The method of marker design discussed in this work can be extended for use in specific applications such as the upcoming cadeveric studies at Carleton University; however, there are several key considerations that must be respected in order for proper characterization of deformation behaviour using the X-ray facility described previously.

The first requirement for proper characterization of brain deformation is the marker visibility. There is a minimum contrast requirement for proper image correlation measurement. The method of contrast determination described in Sec. 4.1 provides a minimum contrast required to achieve a suitable error bound. It should be noted that in practice, increasing the contrast beyond this lower limit may be preferred in the case of non-repeatable sample preparation. As such, a range of marker compositions should be imaged through cadaveric specimens to confirm optimal marker design before embedding in the brains.

One of the single most important factors in measurement accuracy is planarity. First, the markers must be placed in a flat plane with very little variability. With cast materials, this is best achieved by waiting for the first half of the specimen to cure almost completely before placing the markers. With PMHS, surgical techniques will be required and the best way to confirm proper marker placement will likely be repeated X-ray images during the procedure. These methods will ensure planarity of markers, but it is also critical that the marker plane remain parallel to the scintillator throughout the deformation. It may not 
be possible to fully prevent out-of-plane motion in certain impact studies, or due to Poisson's ratio causing out-of-plane deformation; however, two methods can be used to help reduce, or at minimum, quantify possible strain errors. First, placing a second high-speed camera parallel to the impact plane may help quantify the out-of-plane motion. Second, implementing a bi-planar X-ray system to increase measurement accuracy, effectively eliminating out-of-plane motion errors should be a priority moving forward. 


\section{List of References}

[1] Wikipedia contributor: Ulflund, "X-ray applications - Wikipedia, the free encyclopedia," 2012. [Online; accessed 31-July-2018].

[2] J. M. Boone, "X-ray Production, Interaction , and Detection in Diagnostic Imaging," in Handbook of Medical Imaging, vol. 1, ch. 1, pp. 3-77, SPIE - The International Society for Optical Engineering, 2000.

[3] J. Prince and J. Links, Medical imaging signals and systems. Upper Saddle River, NJ: Pearson, 2 ed., 2006.

[4] A. Londe, "X-ray 1896 - nouvelle iconographie de la salptrire - Wikipedia, the free encyclopedia," 2012. [Online; accessed 31-July-2018].

[5] "Biomedical imaging and therapy (bmit) - tech info - technology overview." https: //bmit.lightsource.ca/tech-info/technology-overview/. [Online; accessed 31July-2018].

[6] Canadian Light Source Inc, "Cls synchrotron - Wikipedia, the free encyclopedia," 2008. [Online; accessed 31-July-2018].

[7] R. Nowotny, "X-ray Production, OIAEA," in Diagnostic Radiology Physics: A Handbook for Teachers and Students (D. Dance, S. Christofides, A. Maidment, I. McLean, and K. Ng, eds.), ch. 5, pp. 89-115, Vienna: International Atomic Energy Agency (IAEA), 2014.

[8] R. Nowotny, "X-ray Production, OIAEA ," in Diagnostic Radiology Physics: A Handbook for Teachers and Students (D. Dance, S. Christofides, A. Maidment, I. McLean, and K. Ng, eds.), ch. 5, p. 93, Vienna: International Atomic Energy Agency (IAEA), 2014.

[9] P. Sprawls, Physical Principles of Medical Imaging. Sprawls, 2 ed., 1993.

[10] Scandiflash, "Scandiflash Systems."

[11] B. G. Cour-Palais, A. J. Piekutowski, K. V. Dahl, and K. L. Poormon, "Analysis of the UDRI Tests on NEXTEL Multi-Shock Shields," Int. J. Impact Engng VoI, vol. 14, pp. 193-204, 1993. 
[12] G. Cervantes, "X-ray tubes," in Technical Fundamentals of Radiology and CT, ch. 5, IOPscience, 2016.

[13] Siemens, "Simulation of X-ray Spectra," 2018.

[14] J. M. Boone and J. A. Seibert, "An accurate method for computer-generating tungsten anode x-ray spectra from 30 to $140 \mathrm{kV}$," Medical Physics, vol. 24, pp. 1661-1670, nov 1997.

[15] M. A. Sutton, J. H. Yan, V. Tiwari, H. W. Schreier, and J.-J. Orteu, "The effect of outof-plane motion on 2D and 3D digital image correlation measurements," Optics and Lasers in Engineering, vol. 46, no. 10, pp. 746-757, 2008.

[16] International Electrotechnical Commission, "IEC 60336: Medical electrical equipment - X-ray tube assemblies for medical diagnosis - Characteristics of focal spots," tech. rep., International Electrotechnical Commission, 2005.

[17] J. Cunningham and D. Dance, "Interactions of Radiation with Matter@IAEA," in Diagnostic Radiology Physics: A Handbook for Teachers and Students (D. Dance, S. Christofides, A. Maidment, I. McLean, and K. Ng, eds.), ch. 6, pp. 11-32, Vienna: International Atomic Energy Agency, 2014.

[18] R. Nowotny, "X-ray Production, OIAEA," in Diagnostic Radiology Physics: A Handbook for Teachers and Students (D. Dance, S. Christofides, A. Maidment, I. McLean, and K. Ng, eds.), ch. 5, p. 96, Vienna: International Atomic Energy Agency (IAEA), 2014.

[19] D. M. Cunha, A. Tomal, and M. E. Poletti, "Physics in Medicine \& Biology Evaluation of scatter-to-primary ratio, grid performance and normalized average glandular dose in mammography by Monte Carlo simulation including interference and energy broadening effects," Physics in Medicine \& Biology Phys. Med. Biol, vol. 55, pp. 4335$4359,2010$.

[20] M. Berger, J. Hubbell, S. Seltzer, J. Chang, J. Coursey, R. Sukumar, D. Zucker, and K. Olsen, “XCOM: Photon Cross Sections Database -," 1998.

[21] S. M. Seltzer, "Calculation of photon mass energy-transfer and mass energyabsorption coefficients.," Radiation research, vol. 136, no. 2, pp. 147-170, 1993.

[22] E. Yoshimura, "X-ray Dosimetry, CIAEA," in Diagnostic Radiology Physics: A Handbook for Teachers and Students (D. Dance, S. Christofides, A. Maidment, I. McLean, and K. Ng, eds.), ch. 3, pp. 89-113, Vienna: International Atomic Energy Agency, 2014.

[23] M. Nikl, "Scintillation detectors for x-rays," Measurement Science and Technology, vol. 17, no. 4, pp. 37-54, 2006. 
[24] P. Synnergren, H. T. Goldrein, and W. G. Proud, "Application of digital speckle photography to flash impact experiments," Applied optics, vol. 38, no. 19, pp. 4030-4036, 1999.

[25] D. Goodenough, "Tomographic Imaging," in Handbook of Medical Imaging, no. 1, ch. 8, pp. 1-43, SPIE - The International Society for Optical Engineering, 1979.

[26] I. A. Cunningham, "Applied Linear-Systems Theory," in Handbook of Medical Imaging, ch. 2, pp. 79-159, SPIE - The International Society for Optical Engineering, 2000.

[27] Wikipedia contributor: Pleriche, "Unmounted strain gauge - Wikipedia, the free encyclopedia," 2017. [Online; accessed 31-July-2018].

[28] G. Cloud, "Unifying concepts in teaching optical methods," Experimental Techniques, vol. 26, no. 2, pp. 31-34, 2002.

[29] G. Cloud, "Optical methods in experimental mechanics - Part 19: Basic strain measurement by geometric moiré," Experimental Techniques, vol. 29, no. 6, pp. 25-27, 2005.

[30] B. Pan, K. Qian, H. Xie, and A. Asundi, "Two-dimensional digital image correlation for in-plane displacement and strain measurement: A review," Measurement Science and Technology, vol. 20, no. 6, pp. 62001-17, 2009.

[31] B. K. Bay, T. S. Smith, D. P. Fyhrie, and M. Saad, "Digital Volume Correlation: Three-dimensional Strain Mapping Using X-ray Tomography," Experimental Mechanics, vol. 39, pp. 217-226, 1999.

[32] X. Wang, J. F. Witz, A. El Bartali, and C. Jiang, "Infrared thermography coupled with digital image correlation in studying plastic deformation on the mesoscale level," Optics and Lasers in Engineering, vol. 86, pp. 264-274, 2016.

[33] J.-S. Affagard, P. Feissel, and S. F. Bensamoun, "Measurement of the quadriceps muscle displacement and strain fields with ultrasound and Digital Image Correlation (DIC) techniques," IRBM, vol. 36, pp. 170-177, 2015.

[34] L. Lu, D. Fan, B. X. Bie, X. X. Ran, M. L. Qi, N. Parab, J. Z. Sun, H. J. Liao, M. C. Hudspeth, B. Claus, K. Fezzaa, T. Sun, W. Chen, X. L. Gong, and S. N. Luo, "Note: Dynamic strain field mapping with synchrotron $\mathrm{X}$-ray digital image correlation," Review of Scientific Instruments, vol. 85, no. 076101, 2014.

[35] H. Bruck, S. McNeill, M. Sutton, and W. Peters III, "Digital Image Correlation Using Newton-Raphson Method of Partial Differential Correction," Experimental Mechanics, vol. 29, pp. 261-267, 1989.

[36] G. Vendroux and W. G. Knauss, "Submicron deformation field measurements: Part 2. Improved digital image correlation," Experimental Mechanics, vol. 38, no. 2, pp. 86-92, 1998. 
[37] B. Pan, "Full-field strain measurement using a two-dimensional Savitzky-Golay digital differentiator in digital image correlation," Optical Engineering, vol. 46, no. 3, p. 033601, 2007.

[38] W. Tong, "An evaluation of digital image correlation criteria for strain mapping applications," Strain, vol. 41, no. 4, pp. 167-175, 2005.

[39] B. Pan, H. Xie, Z. Wang, K. Qian, and Z. Wang, "Study on subset size selection in digital image correlation for speckle patterns," Optics Express, vol. 16, no. 10, p. 7037, 2008.

[40] S. Yaofeng and J. H. L. Pang, "Study of optimal subset size in digital image correlation of speckle pattern images," Optics and Lasers in Engineering, vol. 45, no. 9, pp. 967-974, 2007.

[41] B. Pan and K. Li, "A fast digital image correlation method for deformation measurement," Optics and Lasers in Engineering, vol. 49, pp. 841-847, 2011.

[42] P. Mazzoleni, F. Matta, E. Zappa, M. A. Sutton, and A. Cigada, “Gaussian pre-filtering for uncertainty minimization in digital image correlation using numerically-designed speckle patterns," Optics and Lasers in Engineering, vol. 66, pp. 19-33, 2018.

[43] N. Lenoir, M. Bornert, J. Desrues, P. Bésuelle, and G. Viggiani, "Volumetric digital image correlation applied to x-ray microtomography images from triaxial compression tests on argillaceous rock," in Strain, vol. 43, pp. 193-205, Wiley/Blackwell (10.1111), aug 2007.

[44] Z. Y. Wang, H. Q. Li, \&. J. W. Tong, and J. T. Ruan, "Statistical Analysis of the Effect of Intensity Pattern Noise on the Displacement Measurement Precision of Digital Image Correlation Using Self-correlated Images," Experimental Mechanics, vol. 47, pp. 701$707,2007$.

[45] Z. Gao, X. Xu, Y. Su, and Q. Zhang, "Experimental analysis of image noise and interpolation bias in digital image correlation," Optics and Lasers in Engineering, vol. 81, pp. 46-53, 2016.

[46] B. Wang and B. Pan, "Subset-based local vs. finite element-based global digital image correlation: A comparison study," Theoretical \& Applied Mechanics Letters, vol. 6, pp. 200-208, 2016.

[47] Light Works, LLC, “Why use telecentric lenses?." http://1w4u.com/index.php/ telecentric-lenses/why-telecentric/. [Online; accessed 31-July-2018].

[48] M. Z. Siddiqui, "Towards eliminating the displacement bias due to out-of-plane motion in 2D inverse problems: A case of general rigid-body motion," Strain, vol. 51, no. 1, pp. 55-70, 2015. 
[49] M. Hudspeth, B. Claus, S. Dubelman, J. Black, A. Mondal, N. Parab, C. Funnell, F. Hai, M. L. Qi, K. Fezzaa, S. N. Luo, and W. Chen, "High speed synchrotron x-ray phase contrast imaging of dynamic material response to split Hopkinson bar loading," Review of Scientific Instruments, vol. 84, p. 25102, 2013.

[50] K. Haldrup, S. F. Nielsen, and J. A. Wert, "A general methodology for full-field plastic strain measurements using X-ray absorption tomography and internal markers," Experimental Mechanics, vol. 48, no. 2, pp. 199-211, 2008.

[51] S. S. Russell and M. A. Sutton, "Strain-field analysis acquired through correlation of X-ray radiographs of a fiber-reinforced composite laminate," Experimental Mechanics, vol. 29, no. 2, pp. 237-240, 1989.

[52] Y. Luo, S. Wu, Y. Hu, and Y. Fu, "High resolution synchrotron-based X-ray microtomography cracking mechanism of aluminum materials: A review," Frontiers of Mechanical Engineering in China, no. December, 2016.

[53] W.-Y. Jang and S. Kyriakides, "On the crushing of aluminum open-cell foams: Part I. Experiments," International Journal of Solids and Structures, vol. 46, 2009.

[54] S. E. Hamdi, R. Moutou Pitti, M. C. Teguedi, B. Odounga, and B. Blaysat, "3D crack growth monitoring in wood-based materials by means of X-ray computed microtomography and 2D DIC analysis method," in 23ème Congrès Français de Mécanique, 2017.

[55] T. Goldrein, S. G. Grantham, G. Proud, and J. E. Field, "the Study of Internal Deformation Fields in Granular Materials Using 3D Digital Speckle X-Ray Flash Photography," in Proc. of SPIE, 25th International Congress on High-Speed Photography and Photonics, vol. 4948, pp. 592-597, SPIE - The International Society for Optical Engineering, 2003.

[56] D. Takano, N. Lenoir, J. Otani, and S. A. Hall, "Localised deformation in a widegrained sand under triaxial compression revealed by $\mathrm{X}$-ray tomography and digital image correlation," Soils and Foundations, vol. 55, no. 4, pp. 906-915, 2015.

[57] N. Bourne, L. Forde, J. Millett, and J. Field, "Impact and Penetration of a Borosilicate Glass," Journal de Physique IV Colloque, no. C3, p. 7, 1997.

[58] E. Strassburger, S. Bauer, S. Weber, and H. Gedon, “Flash X-ray cinematography analysis of dwell and penetration of small caliber projectiles with three types of $\mathrm{SiC}$ ceramics," Defence Technology, vol. 12, no. 3, pp. 277-283, 2016.

[59] A. S. Iwaskiw, K. A. Ott, R. S. Armiger, A. C. Wickwire, V. D. Alphonse, L. M. Voo, C. M. Carneal, and A. C. Merkle, "The measurement of intracranial pressure and brain displacement due to short-duration dynamic overpressure loading," Shock Waves, vol. 28, pp. 63-83, 2018. 
[60] W. N. Hardy, M. J. Mason, C. D. Foster, C. S. Shah, J. M. Kopacz, K. H. Yang, A. I. King, J. Bishop, M. Bey, W. Anderst, and S. Tashman, "A Study of the Response of the Human Cadaver Head to Impact," Stapp car crash journal, vol. 51, pp. 17-80, 2007.

[61] S. Y. Fu, X. Q. Feng, B. Lauke, and Y. W. Mai, “Effects of particle size, particle/matrix interface adhesion and particle loading on mechanical properties of particulatepolymer composites," Composites Part B: Engineering, vol. 39, no. 6, pp. 933-961, 2008.

[62] J. M. Boone, T. R. Fewell, and R. J. Jennings, “Molybdenum, rhodium, and tungsten anode spectral models using interpolating polynomials with application to mammography," Medical Physics, vol. 24, no. 12, pp. 1863-1874, 1997.

[63] R. Kramer, H. J. Khoury, and J. W. Vieira, “CALDose_X - A software tool for the assessment of organ and tissue absorbed doses, effective dose and cancer risks in diagnostic radiology," Physics in Medicine and Biology, vol. 53, no. 22, pp. 6437-6459, 2008.

[64] Edmund Optics, "Understanding focal length and field of view." https://www.edmundoptics.com/resources/application-notes/imaging/ understanding-focal-length-and-field-of-view/. [Online; accessed 31July-2018].

[65] DXOMARK Image Labs, "Explore lens database." https://www.dxomark. com/lenses/brand-nikon/launched-between-1987-and-2017/mount_ type-Nikon_F_DX-Nikon_F_FX/aperture_max-from-0.95-to-1.4/launch_ price-from-0-to-13000-usd/lens_zoom-prime/sensor_brand-Nikon\# hideAdvancedOptions=false\&viewMode=list\&yDataType=global. [Online; accessed 31-July-2018].

[66] V. B. Mikhailik, V. Kapustyanyk, V. Tsybulskyi, V. Rudyk, and H. Kraus, "Luminescence and scintillation properties of CsI-a potential cryogenic scintillator," tech. rep., Cornell University, 2014.

[67] J. Yorkston and J. a. Rowlands, "Flat Panel Detectors for Digital Radiography," in Handbook of Medical Imaging, ch. 4, pp. 1-102, SPIE - The International Society for Optical Engineering.

[68] Wikipedia contributor: Kieranmaher, "Heeleffect - Wikipedia, the free encyclopedia," 2001. [Online; accessed 31-July-2018].

[69] R. Nowotny, "X-ray Production, CIAEA ," in Diagnostic Radiology Physics: A Handbook for Teachers and Students (D. Dance, S. Christofides, A. Maidment, I. McLean, and K. Ng, eds.), ch. 5, p. 97, Vienna: International Atomic Energy Agency (IAEA), 2014.

[70] Z. I. Kalcioglu, R. A. Mrozek, R. Mahmoodian, M. R. VanLandingham, J. L. Lenhart, and K. J. Van Vliet, "Tunable mechanical behavior of synthetic organogels as biofidelic tissue simulants," Journal of Biomechanics, vol. 46, no. 9, pp. 1583-1591, 2013. 
[71] PubChem, "PubChem Compound 24414," tech. rep., National Center for Biotechnology Information., 2012.

[72] S. Ouellet and M. Philippens, "The multi-modal responses of a physical head model subjected to various blast exposure conditions," Shock Waves, vol. 28, p. 1, jan 2017.

[73] J. Blaber, B. Adair, and A. Antoniou, "Ncorr: Open-Source 2D Digital Image Correlation Matlab Software," Experimental Mechanics, vol. 55, pp. 1105-1122, 2015.

[74] NOCSAE, "Standard Test Method and Equipment Used in Evaluating the Performance Characteristics of Protective Headgear / Equipment NOCSAE DOC ( ND ) 001- 11m13 National Operating Committee," Tech. Rep. February, 2017.

[75] Wikipedia contributor: Dscraggs, "Klein-nishina distribution - Wikipedia, the free encyclopedia," 2009. [Online; accessed 31-July-2018]. 


\section{Appendix A}

\section{X-ray-Matter Interactions}

X-rays incident on a material have interaction probabilities based on wavelength, material density, composition and thickness. At energy levels below $1.02 \mathrm{MeV}$, Rayleigh scattering, Compton scattering and the photoelectric effect occur [2] . Supplemental descriptions of these physical processes is provided in this section.

\section{A.1 Rayleigh (Coherent) Scatter}

An incident X-ray wave can interact with a whole atom simultaneously resulting in Rayleigh scattering. This is an elastic process by which the incident wave is absorbed by the atom, thereby causing synchronized electron vibration. The photon is instantaneously reimmited at the same energy levels, but on a deviated trajectory [2] .

The contributions of Rayleigh scattering above $70 \mathrm{keV}$ are below $5 \%$ when considering all types of scatter. [2] Importantly, Rayleigh scatter decreases image quality because reemmited waves have not lost energy, but have a different trajectory, and could still reach the sensor to provide incorrect information. 


\section{A.2 Compton (Incoherent) Scatter}

Compton scattering is a process whereby a photon havin energy $E_{0}$ will interact directly with an electron, resulting in ionization [2] . Sufficient energy is transfered such that the electron will also gain kinetic energy (which will be lost as heat). This process does not consume the entirety of the photon energy, therefore the incident X-ray photon trajectory will be deviated and energy level reduced to $E^{\prime}$ (as the electron absorbed a portion of its energy) [2].

The Compton process described above can result in significant image noise. The scattered photons tend to continue in a deviated forward trajectory, with less deviation at higher energy levels, making the photons more likely to reach the sensor, increasing noise [2] . This behaviour is governed by the Klein-Nishina formula, which is derived from energy and moment balance equations using the electron mass, $m_{e}$, and the speed of light, $c[2]$.

$$
E^{\prime}=\frac{E_{0}}{\left(1+E_{0} / m_{e} c^{2}\right)(1-\cos \theta)}
$$

As a direct result of Eq. A.1 higher energy photons are more likely to scatter at small, forward angles. High energy photons also have a lower probability of interacting with atoms or electrons. The deviated photons are thus more likely to continue interacting with other electrons/atoms due to their lower energy states [2]. Fig. A.1 shows a radial plot of the probabilistic emission direction of photons by the Compton process for different incident energy levels.

Electron density is the most important factor influencing the probability of Compton scattering [2]. As the quantity of electrons present in a material increases, the likelihood of photon-electron interactions occuring also increases. In non-hydrogenous materials, the electron density per unit mass or material remains relatively constant (ie. not directly dependent on the atomic number), while it is approximately equal to material density per unit volume. Hydrogeneous materials have smaller nuclei absent of neutrons, therefore 


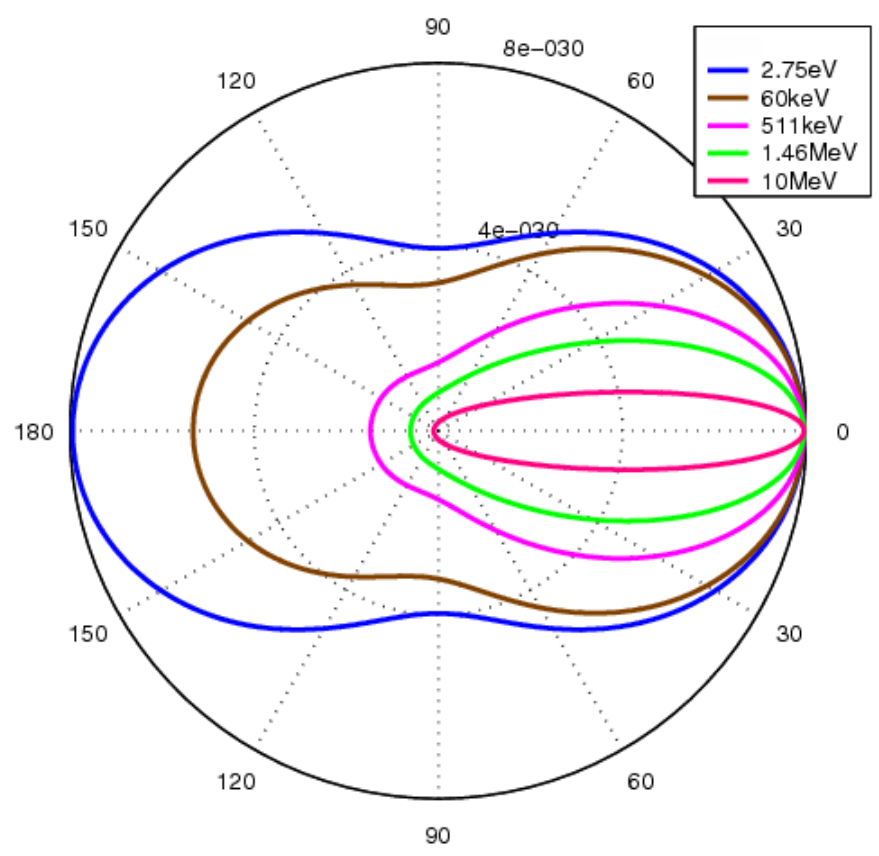

Figure A.1: Illustration of Compton scatter probabilistic emission direction at several power levels [75].

approximately the electron density can be double that of a non-hydogeneous material [2]. The probability of compton scatter can be approximated as constant for photon energies below $100 \mathrm{keV}$ and proportional to 1/E when $\mathrm{E}>300 \mathrm{keV}$ [2].

\section{A.3 Photoelectric Effect}

A special case of photon-electron interaction is photoelastic absorption. In this situation, much like for Compton scattering, a photon transfers energy to an electron; however, the photoelectric effect describes the process by which a photon transfers all of its energy to the electron, overcoming the atomic bonding energy of the nucleus, with any remaining energy transfered as kinetic energy [2]. As the primary photon is fully consumed by the photoelectric effect, there is no direct scatter and therefore no negative effect on image 
quality.

The photoelectric effect is more likely to occur when the photon energy is close to the binding energy of an electron [2]. Although significantly less likely, a photon could interact with an electron having a significantly lower energy, resulting in ionization of an electron with a high kinetic energy. In this case, the electron is most likely to lose its energy as dissipated heat, but $X$-rays following a bremsstrahlung could be generated after secondary interactions with matter [2]. Additionally, the ejected electron leaves an empty space in an internal orbital, therefore an upper orbital electron must take its place. A photon will be emmitted from this process, however it is typically much lower than the incident beam photon levels and would not influence noise levels. The probability of the photoelastic effect occuring is approximately proportional to the cube of the atomic number and the inverse of incident photon energy as shown [2]:

$$
P \alpha Z_{a}^{3} / E^{3}
$$




\section{Appendix B}

\section{Out-of-Plane Motion: Error Derivation}

This chapter describes the derivation of an analytical model used to quantify error of a strained element experiencing out-of-plane motion (translation along $z$ and biaxial rotations about $x$ and $y$ ) shown in Fig. B.1.

The out-of-plane motion uncertainty analysis was performed on a strain element with initial position:

$$
P=(x, y, z)
$$

The points are linearly magnified onto the scintillator plane at Z:

$$
Q=\left(P_{x} \frac{Z}{P_{z}}, P_{y} \frac{Z}{P_{z}}\right)
$$

In the context of DIC, a scaling factor, measured in units/pixel, is used to quantify image

displacements. This scaling factor has effective value of $\frac{Z}{z}$, therefore, point $P$ corresponds to point $R$ on the image is:

$$
R=\left(Q_{x} \frac{z}{Z}, Q_{y} \frac{z}{Z}\right)=\left(P_{x} \frac{z}{P_{z}}, P_{y} \frac{z}{P_{z}}\right)
$$




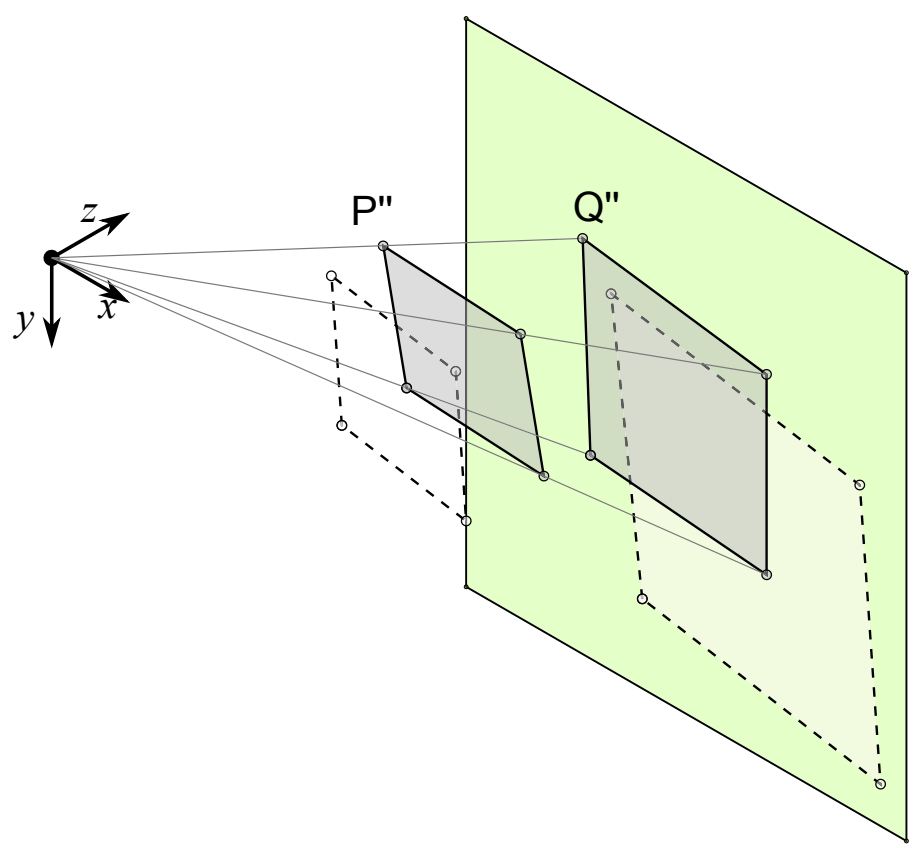

Figure B.1: Projection of the strained element after out-of-plane translation and biaxial rotation.

The infinitesimal strain element is subjected to a first-order deformation (Fig. B.2) such that

$$
\begin{aligned}
& P_{x}^{\prime}-P_{x}=u_{1}(x, y)=u(x, y)+\frac{\partial u}{\partial x} d x+\frac{\partial u}{\partial y} d y \\
& P_{y}^{\prime}-P_{y}=v_{1}(x, y)=v(x, y)+\frac{\partial v}{\partial x} d x+\frac{\partial v}{\partial y} d y
\end{aligned}
$$

the corresponding scaled image deformations are identically:

$$
\begin{aligned}
& R_{x}^{\prime}-R_{x}=u_{1}(x, y)=u(x, y)+\frac{\partial u}{\partial x} d x+\frac{\partial u}{\partial y} d y \\
& R_{y}^{\prime}-R_{y}=v_{1}(x, y)=v(x, y)+\frac{\partial v}{\partial x} d x+\frac{\partial v}{\partial y} d y
\end{aligned}
$$

From Fig. B.2 and Eq. 16 and 17, the associated first-order strains (i.e., displacement gradients) become the following: 


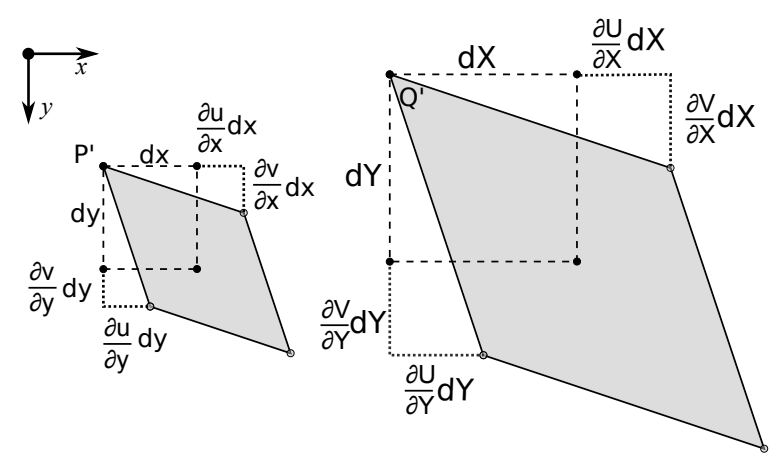

Figure B.2: Infinitesimal strain element and projection definitions

$$
\begin{array}{r}
\varepsilon_{x x}=\frac{\partial u}{\partial x} \\
\varepsilon_{y y}=\frac{\partial v}{\partial y} \\
\varepsilon_{x y} \approx \tan \varepsilon_{x y}=\frac{\frac{\partial u}{\partial y}}{1+\frac{\partial v}{\partial y}} \\
\varepsilon_{y x} \approx \tan \varepsilon_{y x}=\frac{\frac{\partial v}{\partial x}}{1+\frac{\partial u}{\partial x}}
\end{array}
$$

Eq. B.4-B.5 define the coordinates deformed using a first order transformation function. In their current configuration, they correspond to the equations that would be solved by a typical DIC algorithm. Out-of-plane motion causes error in computed strain by altering the form of image points from Eq. B.4-B.5 to a form that will be misinterpreted by firstorder DIC algorithms. C

Complete out-of-plane motion is characterized by translation in along the $z^{\prime}$ axis, and rotation about the $x^{\prime}$ and $y^{\prime}$ axes. For completeness, rotation about the $z^{\prime}$ axis is also included in the model. The rotation axes, $x^{\prime}, y^{\prime}$ and $z^{\prime}$ axes are parallel to $x, y$ and $z$, intersect at point $O_{r}$ assumed to be at an arbitrary point on the $z$ plane $\left(O_{x}, O_{y}, z\right)$. It is noted that any out-of-plane motion can be expressed as a rotation about a single point followed by a translation. The equations are derived in a manner that is condusive to use with rotation and/or translation to match experimental needs. 
The transformation order is assumed to be application of first order deformation, rotation about the $z^{\prime}$ axis, rotation about the $y^{\prime}$ axis, rotation about the $x^{\prime}$ axis, then translation along the $z^{\prime}$ axis. The general form of the transformation is the following:

$$
\left\{P_{i}^{\prime \prime}\right\}=\left[R_{z y x}\right]\left\{P_{i}^{\prime}-O_{r}\right\}+\left\{O_{r}+\Delta z\right\}
$$

where $R_{z y x}$ is the general rotation matrix about the $z^{\prime}, y^{\prime}$, then $x^{\prime}$ axes. $R_{z y x}$ is the result of pre-multiplying coordinates by individual rotation matrices $R_{z}, R_{y}$, then $R_{x}$, giving the form:

$$
\left[\begin{array}{ccc}
\cos \theta_{y} \cos \theta_{z} & -\cos \theta_{y} \sin \theta_{z} & \sin \theta_{y} \\
\cos \theta_{x} \sin \theta_{z}+\cos \theta_{z} \sin \theta_{x} \sin \theta_{y} & \cos \theta_{x} \cos \theta_{z}-\sin \theta_{x} \sin \theta_{y} \sin \theta_{z} & -\cos \theta_{y} \sin \theta_{x} \\
\sin \theta_{x} \sin \theta_{z}-\cos \theta_{x} \cos \theta_{z} \sin \theta_{y} & \cos \theta_{z} \sin \theta_{x}+\cos \theta_{x} \sin \theta_{y} \sin \theta_{z} & \cos \theta_{x} \cos \theta_{y}
\end{array}\right]
$$

Alternately expressed as:

$$
R_{z y x}=\left[\begin{array}{lll}
a_{11} & a_{12} & a_{13} \\
a_{21} & a_{22} & a_{23} \\
a_{31} & a_{32} & a_{33}
\end{array}\right]
$$

The deformed coordinates of point $P$ after first order deformation and out-of-plane motion becomes $P^{\prime \prime}$ : 


$$
\begin{array}{r}
\left\{P^{\prime \prime}\right\}=\left(\left(x+u_{1}-O_{x}\right) a_{11}+\left(y+v_{1}-O_{y}\right) a_{12}+O_{x},\right. \\
\left(x+u_{1}-O_{x}\right) a_{21}+\left(y+v_{1}-O_{y}\right) a_{22}+O_{y}, \\
\left.z+\Delta z+\left(x+u_{1}-O_{x}\right) a_{31}+\left(y+v_{1}-O_{y}\right) a_{32}\right)
\end{array}
$$

As shown in Eq. B.6 and B.7, the location of the object in the image depends on the current and original position along the $z$ axis relative to the source and scintillator. The transformation between object and image coordinates now becomes:

$$
\begin{aligned}
& R_{x}^{\prime \prime}=P_{x}^{\prime \prime} \frac{Z}{P_{z}^{\prime \prime}} \frac{P_{z}}{Z}=P_{x}^{\prime \prime} \frac{z}{P_{i, z}^{\prime \prime}} \\
& R_{y}^{\prime \prime}=P_{y}^{\prime \prime} \frac{Z}{P_{z}^{\prime \prime}} \frac{P_{z}}{Z}=P_{y}^{\prime \prime} \frac{z}{P_{z}^{\prime \prime}}
\end{aligned}
$$

which evaluates to the following:

$$
\begin{aligned}
\left\{R^{\prime \prime}\right\}= & \left(\frac{z\left(\left(x+u_{1}-O_{x}\right) a_{11}+\left(y+v_{1}-O_{y}\right) a_{12}+O_{x}\right)}{z+\Delta z+\left(x+u_{1}-O_{x}\right) a_{31}+\left(y+v_{1}-O_{y}\right) a_{32}},\right. \\
& \left.\frac{z\left(\left(x+u_{1}-O_{x}\right) a_{21}+\left(y+v_{1}-O_{y}\right) a_{22}+O_{y}\right)}{z+\Delta z+\left(x+u_{1}-O_{x}\right) a_{31}+\left(y+v_{1}-O_{y}\right) a_{32}}\right)
\end{aligned}
$$

The perceived deformation between the original and transformed image points is:

$$
\begin{aligned}
& R_{x}^{\prime \prime}-R_{x}=u_{1}^{\prime}(x, y) \\
& R_{y}^{\prime \prime}-R_{y}=v_{1}^{\prime}(x, y)
\end{aligned}
$$

with the associated perceived strains expressed as 


$$
\begin{gathered}
\varepsilon_{x x}^{\prime}=\frac{\partial u_{1}^{\prime}}{\partial x} \\
\varepsilon_{y y}^{\prime}=\frac{\partial v_{1}^{\prime}}{\partial y} \\
\varepsilon_{x y}^{\prime}=\frac{\frac{\partial u_{1}^{\prime}}{\partial y}}{1+\frac{\partial v_{1}^{\prime}}{\partial y}} \\
\varepsilon_{y x}^{\prime}=\frac{\frac{\partial v_{1}^{\prime}}{\partial x}}{1+\frac{\partial u_{1}^{\prime}}{\partial x}}
\end{gathered}
$$

The displacements expand to:

$$
\begin{aligned}
& u_{1}^{\prime}(x, y)=\frac{z\left(\left(x+u_{1}-O_{x}\right) a_{11}+\left(y+v_{1}-O_{y}\right) a_{12}+O_{x}\right)}{z+\Delta z+\left(x+u_{1}-O_{x}\right) a_{31}+\left(y+v_{1}-O_{y}\right) a_{32}}-x \\
& v_{1}^{\prime}(x, y)=\frac{z\left(\left(x+u_{1}-O_{x}\right) a_{21}+\left(y+v_{1}-O_{y}\right) a_{22}+O_{y}\right)}{z+\Delta z+\left(x+u_{1}-O_{x}\right) a_{31}+\left(y+v_{1}-O_{y}\right) a_{32}}-y
\end{aligned}
$$

The awful next step, taking the derivative to determine the perceived strains probably gives the following, written here first as the partial derivatives of termwise numerators and denominators seperately, ignoring higher order terms:

$u_{1}^{\prime}(x, y) 1$ st term, numerator:

$$
\begin{aligned}
& \frac{\partial}{\partial x}=z\left(\left(1+\frac{\partial u_{1}}{\partial x}\right) a_{11}+\left(\frac{\partial v_{1}}{\partial x}\right) a_{12}\right) \\
& \frac{\partial}{\partial y}=z\left(\left(\frac{\partial u_{1}}{\partial y}\right) a_{11}+\left(1+\frac{\partial v_{1}}{\partial y}\right) a_{12}\right)
\end{aligned}
$$


$v_{1}^{\prime}(x, y) 1$ st term, numerator:

$$
\begin{aligned}
& \frac{\partial}{\partial x}=z\left(\left(1+\frac{\partial u_{1}}{\partial x}\right) a_{21}+\left(\frac{\partial v_{1}}{\partial x}\right) a_{22}\right) \\
& \frac{\partial}{\partial y}=z\left(\left(\frac{\partial u_{1}}{\partial y}\right) a_{21}+\left(1+\frac{\partial v_{1}}{\partial y}\right) a_{22}\right)
\end{aligned}
$$

Common 1st term denominator:

$$
\begin{aligned}
& \frac{\partial}{\partial x}=\left(\left(1+\frac{\partial u_{1}}{\partial x}\right) a_{31}+\left(\frac{\partial v_{1}}{\partial x}\right) a_{32}\right) \\
& \frac{\partial}{\partial y}=\left(\left(\frac{\partial u_{1}}{\partial y}\right) a_{31}+\left(1+\frac{\partial v_{1}}{\partial y}\right) a_{32}\right)
\end{aligned}
$$

The first-order derivatives and strains are found to be:

$$
\begin{aligned}
& \frac{\partial u_{1}^{\prime}}{\partial x}=\varepsilon_{x x}^{\prime}= \\
& \left(\left(1+\frac{\partial u_{1}}{\partial x}\right) a_{11}+\left(\frac{\partial v_{1}}{\partial x}\right) a_{12}\right)\left(z+\Delta z+\left(x+u_{1}-O_{x}\right) a_{31}+\left(y+v_{1}-O_{y}\right) a_{32}\right) \\
& z \frac{-\left(\left(x+u_{1}-O_{x}\right) a_{11}+\left(y+v_{1}-O_{y}\right) a_{12}+O_{x}\right)\left(\left(1+\frac{\partial u_{1}}{\partial x}\right) a_{31}+\left(\frac{\partial v_{1}}{\partial x}\right) a_{32}\right)}{\left(z+\Delta z+\left(x+u_{1}-O_{x}\right) a_{31}+\left(y+v_{1}-O_{y}\right) a_{32}\right)^{2}}-1 \\
& \left(1+\frac{\partial u_{1}}{\partial x}\right)\left((z+\Delta z) a_{11}+\left(y+v_{1}-O_{y}\right)\left(a_{11} a_{32}-a_{12} a_{31}\right)-a_{31} O_{x}\right) \\
& =z \frac{+\left(\frac{\partial v_{1}}{\partial x}\right)\left((z+\Delta z) a_{12}+\left(x+u_{1}-O_{x}\right)\left(a_{12} a_{31}-a_{11} a_{32}\right)-a_{32} O_{x}\right)}{\left(z+\Delta z+\left(x+u_{1}-O_{x}\right) a_{31}+\left(y+v_{1}-O_{y}\right) a_{32}\right)^{2}}-1
\end{aligned}
$$




$$
\begin{aligned}
& \frac{\partial u_{1}^{\prime}}{\partial y}= \\
& \left(\left(\frac{\partial u_{1}}{\partial y}\right) a_{11}+\left(1+\frac{\partial v_{1}}{\partial y}\right) a_{12}\right)\left(z+\Delta z+\left(x+u_{1}-O_{x}\right) a_{31}+\left(y+v_{1}-O_{y}\right) a_{32}\right) \\
& z \frac{-\left(\left(x+u_{1}-O_{x}\right) a_{11}+\left(y+v_{1}-O_{y}\right) a_{12}+O_{x}\right)\left(\left(\frac{\partial u_{1}}{\partial y}\right) a_{31}+\left(1+\frac{\partial v_{1}}{\partial y}\right) a_{32}\right)}{\left(z+\Delta z+\left(x+u_{1}-O_{x}\right) a_{31}+\left(y+v_{1}-O_{y}\right) a_{32}\right)^{2}} \\
& \quad+\left(1+\frac{\partial v_{1}}{\partial y}\right)\left((z+\Delta z) a_{12}+\left(x+u_{1}-O_{x}\right)\left(a_{12} a_{31}-a_{11} a_{32}\right)-a_{32} O_{x}\right) \\
& \left(z+\Delta z+\left(x+u_{1}-O_{x}\right) a_{31}+\left(y+v_{1}-O_{y}\right) a_{32}\right)^{2}
\end{aligned}
$$

$$
\begin{gathered}
\frac{\partial v_{1}^{\prime}}{\partial x}= \\
\begin{array}{c}
\left(\left(1+\frac{\partial u_{1}}{\partial x}\right) a_{21}+\left(\frac{\partial v_{1}}{\partial x}\right) a_{22}\right)\left(z+\Delta z+\left(x+u_{1}-O_{x}\right) a_{31}+\left(y+v_{1}-O_{y}\right) a_{32}\right) \\
-\left(\left(x+u_{1}-O_{x}\right) a_{11}+\left(y+v_{1}-O_{y}\right) a_{22}+O_{y}\right)\left(\left(1+\frac{\partial u_{1}}{\partial x}\right) a_{31}+\left(\frac{\partial v_{1}}{\partial x}\right) a_{32}\right)
\end{array} \\
\left(z+\Delta z+\left(x+u_{1}-O_{x}\right) a_{31}+\left(y+v_{1}-O_{y}\right) a_{32}\right)^{2} \\
\quad z \frac{\left(1+\frac{\partial u_{1}}{\partial x}\right)\left((z+\Delta z) a_{21}+\left(y+v_{1}-O_{y}\right)\left(a_{21} a_{32}-a_{22} a_{31}\right)-a_{31} O_{y}\right)}{\left(z+\Delta z+\left(x+u_{1}-O_{x}\right) a_{31}+\left(y+v_{1}-O_{y}\right) a_{32}\right)^{2}}
\end{gathered}
$$




$$
\begin{aligned}
& \frac{\partial v_{1}^{\prime}}{\partial y}=\varepsilon_{y y}^{\prime}= \\
& \left(\left(\frac{\partial u_{1}}{\partial y}\right) a_{21}+\left(1+\frac{\partial v_{1}}{\partial y}\right) a_{22}\right)\left(z+\Delta z+\left(x+u_{1}-O_{x}\right) a_{31}+\left(y+v_{1}-O_{y}\right) a_{32}\right) \\
& z \frac{-\left(\left(x+u_{1}-O_{x}\right) a_{21}+\left(y+v_{1}-O_{y}\right) a_{22}+O_{y}\right)\left(\left(\frac{\partial u_{1}}{\partial y}\right) a_{31}+\left(1+\frac{\partial v_{1}}{\partial y}\right) a_{32}\right)}{\left(z+\Delta z+\left(x+u_{1}-O_{x}\right) a_{31}+\left(y+v_{1}-O_{y}\right) a_{32}\right)^{2}}-1 \\
& \quad+\left(1+\frac{\partial v_{1}}{\partial y}\right)\left((z+\Delta z) a_{22}+\left(x+u_{1}-O_{x}\right)\left(a_{22} a_{31}-a_{21} a_{32}\right)+a_{32} O_{y}\right) \\
& \left(z+\Delta z+\left(x+u_{1}-O_{x}\right) a_{31}+\left(y+v_{1}-O_{y}\right) a_{32}\right)^{2}
\end{aligned}
$$

$\varepsilon_{x y}^{\prime}=$

$$
\begin{aligned}
& \left(\frac{\partial u_{1}}{\partial y}\right)\left((z+\Delta z) a_{11}+\left(y+v_{1}-O_{y}\right)\left(a_{11} a_{32}-a_{12} a_{31}\right)-a_{31} O_{x}\right) \\
& +\left(1+\frac{\partial v_{1}}{\partial y}\right)\left((z+\Delta z) a_{12}+\left(x+u_{1}-O_{x}\right)\left(a_{12} a_{31}-a_{11} a_{32}\right)-a_{32} O_{x}\right) \\
& \left(\frac{\partial u_{1}}{\partial y}\right)\left((z+\Delta z) a_{21}+\left(y+v_{1}-O_{y}\right)\left(a_{21} a_{32}-a_{22} a_{31}\right)+a_{31} O_{y}\right) \\
& \quad+\left(1+\frac{\partial v_{1}}{\partial y}\right)\left((z+\Delta z) a_{22}+\left(x+u_{1}-O_{x}\right)\left(a_{22} a_{31}-a_{21} a_{32}\right)+a_{32} O_{y}\right)
\end{aligned}
$$

$$
\begin{aligned}
\varepsilon_{y x}^{\prime}= & \\
& \begin{aligned}
(1+ & \left.\frac{\partial u_{1}}{\partial x}\right)\left((z+\Delta z) a_{21}+\left(y+v_{1}-O_{y}\right)\left(a_{21} a_{32}-a_{22} a_{31}\right)-a_{31} O_{y}\right) \\
& +\left(\frac{\partial v_{1}}{\partial x}\right)\left((z+\Delta z) a_{22}+\left(x+u_{1}-O_{x}\right)\left(a_{22} a_{31}-a_{21} a_{32}\right)-a_{32} O_{y}\right) \\
& \quad+\left(\frac{\partial v_{1}}{\partial x}\right)\left((z+\Delta z) a_{12}+\left(x+u_{1}-O_{x}\right)\left(a_{12} a_{31}-a_{11} a_{32}\right)-a_{32} O_{x}\right)
\end{aligned}
\end{aligned}
$$


The following is left as an exercise in algebra to the passionate lector, and in trust to the less enthused reader:

\section{Case 0: General}

$$
\begin{aligned}
& a_{11}=\cos \theta_{y} \cos \theta_{z} \\
& a_{12}=-\cos \theta_{y} \sin \theta_{z} \\
& a_{21}=\cos \theta_{x} \sin \theta_{z}+\cos \theta_{z} \sin \theta_{x} \sin \theta_{y} \\
& a_{22}=\cos \theta_{x} \cos \theta_{z}-\sin \theta_{x} \sin \theta_{y} \sin \theta_{z} \\
& a_{31}=\sin \theta_{x} \sin \theta_{z}-\cos \theta_{x} \cos \theta_{z} \sin \theta_{y} \\
& a_{32}=\cos \theta_{z} \sin \theta_{x}+\cos \theta_{x} \sin \theta_{y} \sin \theta_{z}
\end{aligned}
$$

$$
\begin{gathered}
\varepsilon_{x x}^{\prime}= \\
=z \frac{\left(1+\frac{\partial u_{1}}{\partial x}\right)\left((z+\Delta z) a_{11}+\left(y+v_{1}-O_{y}\right)\left(a_{11} a_{32}-a_{12} a_{31}\right)-a_{31} O_{x}\right)}{\left(z+\Delta z+\left(x+u_{1}-O_{x}\right) a_{31}+\left(y+v_{1}-O_{y}\right) a_{32}\right)^{2}}-1 \\
\varepsilon_{y y}^{\prime}=\quad \begin{array}{c}
\left(\frac{\partial u_{1}}{\partial y}\right)\left((z+\Delta z) a_{21}+\left(y+v_{1}-O_{y}\right)\left(a_{21} a_{32}-a_{22} a_{31}\right)+a_{31} O_{y}\right) \\
\quad+\left(1+\frac{\partial v_{1}}{\partial y}\right)\left((z+\Delta z) a_{22}+\left(x+u_{1}-O_{x}\right)\left(a_{22} a_{31}-a_{21} a_{32}\right)+a_{32} O_{y}\right)
\end{array}-1 \\
\left(z+\Delta z+\left(x+u_{1}-O_{x}\right) a_{31}+\left(y+v_{1}-O_{y}\right) a_{32}\right)^{2}
\end{gathered}
$$




$$
\begin{aligned}
\varepsilon_{x y}^{\prime}= & \\
& \begin{aligned}
&\left.\frac{\partial u_{1}}{\partial y}\right)\left((z+\Delta z) a_{11}+\left(y+v_{1}-O_{y}\right)\left(a_{11} a_{32}-a_{12} a_{31}\right)-a_{31} O_{x}\right) \\
& \quad+\left(1+\frac{\partial v_{1}}{\partial y}\right)\left((z+\Delta z) a_{12}+\left(x+u_{1}-O_{x}\right)\left(a_{12} a_{31}-a_{11} a_{32}\right)-a_{32} O_{x}\right) \\
& \frac{\left(\frac{\partial u_{1}}{\partial y}\right)\left((z+\Delta z) a_{21}+\left(y+v_{1}-O_{y}\right)\left(a_{21} a_{32}-a_{22} a_{31}\right)+a_{31} O_{y}\right)}{} \\
&+\left(1+\frac{\partial v_{1}}{\partial y}\right)\left((z+\Delta z) a_{22}+\left(x+u_{1}-O_{x}\right)\left(a_{22} a_{31}-a_{21} a_{32}\right)+a_{32} O_{y}\right)
\end{aligned}
\end{aligned}
$$

$$
\varepsilon_{y x}^{\prime}=
$$

$$
\begin{aligned}
\left(1+\frac{\partial u_{1}}{\partial x}\right)\left((z+\Delta z) a_{21}+\left(y+v_{1}-O_{y}\right)\left(a_{21} a_{32}-a_{22} a_{31}\right)-a_{31} O_{y}\right) \\
+\left(\frac{\partial v_{1}}{\partial x}\right)\left((z+\Delta z) a_{22}+\left(x+u_{1}-O_{x}\right)\left(a_{22} a_{31}-a_{21} a_{32}\right)-a_{32} O_{y}\right) \\
\left(1+\frac{\partial u_{1}}{\partial x}\right)\left((z+\Delta z) a_{11}+\left(y+v_{1}-O_{y}\right)\left(a_{11} a_{32}-a_{12} a_{31}\right)-a_{31} O_{x}\right) \\
+\left(\frac{\partial v_{1}}{\partial x}\right)\left((z+\Delta z) a_{12}+\left(x+u_{1}-O_{x}\right)\left(a_{12} a_{31}-a_{11} a_{32}\right)-a_{32} O_{x}\right)
\end{aligned}
$$

Case 1: $\theta_{x}=0$

$$
\begin{aligned}
& a_{11}=\cos \theta_{y} \cos \theta_{z} \\
& a_{12}=-\cos \theta_{y} \sin \theta_{z} \\
& a_{21}=\sin \theta_{z} \\
& a_{22}=\cos \theta_{z} \\
& a_{31}=-\cos \theta_{z} \sin \theta_{y} \\
& a_{32}=\sin \theta_{y} \sin \theta_{z}
\end{aligned}
$$




$$
\varepsilon_{x x}^{\prime}=
$$

$$
\begin{gathered}
\left(1+\frac{\partial u_{1}}{\partial x}\right)\left((z+\Delta z) a_{11}+\left(y+v_{1}-O_{y}\right)\left(a_{11} a_{32}-a_{12} a_{31}\right)-a_{31} O_{x}\right) \\
=z \frac{+\left(\frac{\partial v_{1}}{\partial x}\right)\left((z+\Delta z) a_{12}+\left(x+u_{1}-O_{x}\right)\left(a_{12} a_{31}-a_{11} a_{32}\right)-a_{32} O_{x}\right)}{\left(z+\Delta z+\left(x+u_{1}-O_{x}\right) a_{31}+\left(y+v_{1}-O_{y}\right) a_{32}\right)^{2}}-1 \\
\varepsilon_{y y}^{\prime}=\quad \\
\left(\frac{\partial u_{1}}{\partial y}\right)\left((z+\Delta z) a_{21}+\left(y+v_{1}-O_{y}\right)\left(a_{21} a_{32}-a_{22} a_{31}\right)+a_{31} O_{y}\right) \\
+\left(1+\frac{\partial v_{1}}{\partial y}\right)\left((z+\Delta z) a_{22}+\left(x+u_{1}-O_{x}\right)\left(a_{22} a_{31}-a_{21} a_{32}\right)+a_{32} O_{y}\right) \\
\left(z+\Delta z+\left(x+u_{1}-O_{x}\right) a_{31}+\left(y+v_{1}-O_{y}\right) a_{32}\right)^{2}
\end{gathered}
$$

$$
\varepsilon_{x y}^{\prime}=
$$

$$
\begin{aligned}
& \left(\frac{\partial u_{1}}{\partial y}\right)\left((z+\Delta z) a_{11}+\left(y+v_{1}-O_{y}\right)\left(a_{11} a_{32}-a_{12} a_{31}\right)-a_{31} O_{x}\right) \\
& +\left(1+\frac{\partial v_{1}}{\partial y}\right)\left((z+\Delta z) a_{12}+\left(x+u_{1}-O_{x}\right)\left(a_{12} a_{31}-a_{11} a_{32}\right)-a_{32} O_{x}\right) \\
& +\left(\frac{\partial u_{1}}{\partial y}\right)\left((z+\Delta z) a_{21}+\left(y+v_{1}-O_{y}\right)\left(a_{21} a_{32}-a_{22} a_{31}\right)+a_{31} O_{y}\right) \\
& +\left(1+\frac{\partial v_{1}}{\partial y}\right)\left((z+\Delta z) a_{22}+\left(x+u_{1}-O_{x}\right)\left(a_{22} a_{31}-a_{21} a_{32}\right)+a_{32} O_{y}\right)
\end{aligned}
$$




$$
\varepsilon_{y x}^{\prime}=
$$

$$
\begin{aligned}
(1+ & \left.\frac{\partial u_{1}}{\partial x}\right)\left((z+\Delta z) a_{21}+\left(y+v_{1}-O_{y}\right)\left(a_{21} a_{32}-a_{22} a_{31}\right)-a_{31} O_{y}\right) \\
& +\left(\frac{\partial v_{1}}{\partial x}\right)\left((z+\Delta z) a_{22}+\left(x+u_{1}-O_{x}\right)\left(a_{22} a_{31}-a_{21} a_{32}\right)-a_{32} O_{y}\right) \\
\left(1+\frac{\partial u_{1}}{\partial x}\right)\left((z+\Delta z) a_{11}+\left(y+v_{1}-O_{y}\right)\left(a_{11} a_{32}-a_{12} a_{31}\right)-a_{31} O_{x}\right) & \\
& +\left(\frac{\partial v_{1}}{\partial x}\right)\left((z+\Delta z) a_{12}+\left(x+u_{1}-O_{x}\right)\left(a_{12} a_{31}-a_{11} a_{32}\right)-a_{32} O_{x}\right)
\end{aligned}
$$

Case 2: $\theta_{y}=0$

$$
\begin{aligned}
& a_{11}=\cos \theta_{z} \\
& a_{12}=-\sin \theta_{z} \\
& a_{21}=\cos \theta_{x} \sin \theta_{z} \\
& a_{22}=\cos \theta_{x} \cos \theta_{z} \\
& a_{31}=\sin \theta_{x} \sin \theta_{z} \\
& a_{32}=\cos \theta_{z} \sin \theta_{x}
\end{aligned}
$$

$$
\begin{gathered}
\varepsilon_{x x}^{\prime}= \\
\quad \begin{array}{c}
\left(1+\frac{\partial u_{1}}{\partial x}\right)\left((z+\Delta z) a_{11}+\left(y+v_{1}-O_{y}\right)\left(a_{11} a_{32}-a_{12} a_{31}\right)-a_{31} O_{x}\right) \\
+\left(\frac{\partial v_{1}}{\partial x}\right)\left((z+\Delta z) a_{12}+\left(x+u_{1}-O_{x}\right)\left(a_{12} a_{31}-a_{11} a_{32}\right)-a_{32} O_{x}\right)
\end{array}-1 \\
\left(z+\Delta z+\left(x+u_{1}-O_{x}\right) a_{31}+\left(y+v_{1}-O_{y}\right) a_{32}\right)^{2}
\end{gathered}
$$




$$
\begin{gathered}
\varepsilon_{y y}^{\prime}= \\
=z \frac{\left(\frac{\partial u_{1}}{\partial y}\right)\left((z+\Delta z) a_{21}+\left(y+v_{1}-O_{y}\right)\left(a_{21} a_{32}-a_{22} a_{31}\right)+a_{31} O_{y}\right)}{+\left(1+\frac{\partial v_{1}}{\partial y}\right)\left((z+\Delta z) a_{22}+\left(x+u_{1}-O_{x}\right)\left(a_{22} a_{31}-a_{21} a_{32}\right)+a_{32} O_{y}\right)}-1 \\
\left(z+\Delta z+\left(x+u_{1}-O_{x}\right) a_{31}+\left(y+v_{1}-O_{y}\right) a_{32}\right)^{2}
\end{gathered}
$$

$\varepsilon_{x y}^{\prime}=$

$$
\begin{aligned}
& \left(\frac{\partial u_{1}}{\partial y}\right)\left((z+\Delta z) a_{11}+\left(y+v_{1}-O_{y}\right)\left(a_{11} a_{32}-a_{12} a_{31}\right)-a_{31} O_{x}\right) \\
& +\left(1+\frac{\partial v_{1}}{\partial y}\right)\left((z+\Delta z) a_{12}+\left(x+u_{1}-O_{x}\right)\left(a_{12} a_{31}-a_{11} a_{32}\right)-a_{32} O_{x}\right) \\
& +\left(\frac{\partial u_{1}}{\partial y}\right)\left((z+\Delta z) a_{21}+\left(y+v_{1}-O_{y}\right)\left(a_{21} a_{32}-a_{22} a_{31}\right)+a_{31} O_{y}\right) \\
& \quad+\left(1+\frac{\partial v_{1}}{\partial y}\right)\left((z+\Delta z) a_{22}+\left(x+u_{1}-O_{x}\right)\left(a_{22} a_{31}-a_{21} a_{32}\right)+a_{32} O_{y}\right)
\end{aligned}
$$

$$
\begin{aligned}
\varepsilon_{y x}^{\prime}= & \\
& \begin{aligned}
(1+ & \left.\frac{\partial u_{1}}{\partial x}\right)\left((z+\Delta z) a_{21}+\left(y+v_{1}-O_{y}\right)\left(a_{21} a_{32}-a_{22} a_{31}\right)-a_{31} O_{y}\right) \\
& \quad+\left(\frac{\partial v_{1}}{\partial x}\right)\left((z+\Delta z) a_{22}+\left(x+u_{1}-O_{x}\right)\left(a_{22} a_{31}-a_{21} a_{32}\right)-a_{32} O_{y}\right) \\
& \quad+\left(1+\frac{\partial u_{1}}{\partial x}\right)\left((z+\Delta z) a_{11}+\left(y+v_{1}-O_{y}\right)\left(a_{11} a_{32}-a_{12} a_{31}\right)-a_{31} O_{x}\right) \\
& \left.\quad(z+\Delta z) a_{12}+\left(x+u_{1}-O_{x}\right)\left(a_{12} a_{31}-a_{11} a_{32}\right)-a_{32} O_{x}\right)
\end{aligned}
\end{aligned}
$$


Case 3: $\theta_{z}=0$

$$
\begin{aligned}
& a_{11}=\cos \theta_{y} \\
& a_{12}=0 \\
& a_{21}=\sin \theta_{x} \sin \theta_{y} \\
& a_{22}=\cos \theta_{x} \\
& a_{31}=-\cos \theta_{x} \sin \theta_{y} \\
& a_{32}=\sin \theta_{x}
\end{aligned}
$$

$$
\begin{aligned}
& \varepsilon_{x x}^{\prime}= \\
& \quad\left(1+\frac{\partial u_{1}}{\partial x}\right)\left((z+\Delta z) a_{11}+\left(y+v_{1}-O_{y}\right)\left(a_{11} a_{32}\right)-a_{31} O_{x}\right) \\
& +\left(\frac{\partial v_{1}}{\partial x}\right)\left(\left(x+u_{1}-O_{x}\right)\left(-a_{11} a_{32}\right)-a_{32} O_{x}\right) \\
& \left(z+\Delta z+\left(x+u_{1}-O_{x}\right) a_{31}+\left(y+v_{1}-O_{y}\right) a_{32}\right)^{2}
\end{aligned}
$$

$$
\begin{aligned}
\varepsilon_{y y}^{\prime}= & \left(\frac{\partial u_{1}}{\partial y}\right)\left((z+\Delta z) a_{21}+\left(y+v_{1}-O_{y}\right)\left(a_{21} a_{32}-a_{22} a_{31}\right)+a_{31} O_{y}\right) \\
= & z \frac{+\left(1+\frac{\partial v_{1}}{\partial y}\right)\left((z+\Delta z) a_{22}+\left(x+u_{1}-O_{x}\right)\left(a_{22} a_{31}-a_{21} a_{32}\right)+a_{32} O_{y}\right)}{\left(z+\Delta z+\left(x+u_{1}-O_{x}\right) a_{31}+\left(y+v_{1}-O_{y}\right) a_{32}\right)^{2}}-1
\end{aligned}
$$




$$
\begin{gathered}
\varepsilon_{x y}^{\prime}= \\
\left.\frac{\partial u_{1}}{\partial y}\right)\left((z+\Delta z) a_{11}+\left(y+v_{1}-O_{y}\right)\left(a_{11} a_{32}\right)-a_{31} O_{x}\right) \\
+\left(1+\frac{\partial v_{1}}{\partial y}\right)\left(\left(x+u_{1}-O_{x}\right)\left(-a_{11} a_{32}\right)-a_{32} O_{x}\right) \\
\quad+\left(1+\frac{\partial u_{1}}{\partial y}\right)\left((z+\Delta z) a_{21}+\left(y+v_{1}-O_{y}\right)\left(a_{21} a_{32}-a_{22} a_{31}\right)+a_{31} O_{y}\right) \\
\left.\varepsilon_{y x}^{\prime}=\quad \begin{array}{r}
\left.(1+\Delta z) a_{22}+\left(x+u_{1}-O_{x}\right)\left(a_{22} a_{31}-a_{21} a_{32}\right)+a_{32} O_{y}\right) \\
+\left(\frac{\partial u_{1}}{\partial x}\right)\left((z+\Delta z) a_{21}+\left(y+v_{1}-O_{y}\right)\left(a_{21} a_{32}-a_{22} a_{31}\right)-a_{31} O_{y}\right) \\
\quad\left(1+\frac{\partial u_{1}}{\partial x}\right)\left((z+\Delta z) a_{22}+\left(x+u_{1}-O_{x}\right)\left(a_{22} a_{31}-a_{21} a_{32}\right)-a_{32} O_{y}\right) \\
\left.+\left(y+v_{1}-O_{y}\right)\left(a_{11} a_{32}\right)-a_{31} O_{x}\right)
\end{array}\right)\left(+\left(x+u_{1}-O_{x}\right)\left(-a_{11} a_{32}\right)-a_{32} O_{x}\right)
\end{gathered}
$$

Case 4: $\theta_{x}=0, \theta_{y}=0$

$$
\begin{aligned}
& a_{11}=\cos \theta_{z} \\
& a_{12}=-\sin \theta_{z} \\
& a_{21}=\sin \theta_{z} \\
& a_{22}=\cos \theta_{z} \\
& a_{31}=0 \\
& a_{32}=0
\end{aligned}
$$




$$
\begin{aligned}
& \varepsilon_{x x}^{\prime}= \\
& =z \frac{\left(1+\frac{\partial u_{1}}{\partial x}\right)\left((z+\Delta z) a_{11}\right)}{\left(\frac{\partial v_{1}}{\partial x}\right)\left((z+\Delta z) a_{12}\right)}(z+\Delta z)^{2}-1 \\
& (z+)^{2}
\end{aligned}
$$

$$
\begin{aligned}
\varepsilon_{y y}^{\prime}= & \left(\frac{\partial u_{1}}{\partial y}\right)\left((z+\Delta z) a_{21}\right) \\
= & z \frac{+\left(1+\frac{\partial v_{1}}{\partial y}\right)\left((z+\Delta z) a_{22}\right)}{(z+\Delta z)^{2}}-1
\end{aligned}
$$

$$
\begin{aligned}
\varepsilon_{x y}^{\prime}= & \\
& \left.\frac{\partial u_{1}}{\partial y}\right)\left((z+\Delta z) a_{11}\right) \\
& +\left(1+\frac{\partial v_{1}}{\partial y}\right)\left((z+\Delta z) a_{12}\right) \\
& \left.\frac{\partial u_{1}}{\partial y}\right)\left((z+\Delta z) a_{21}\right) \\
& +\left(1+\frac{\partial v_{1}}{\partial y}\right)\left((z+\Delta z) a_{22}\right)
\end{aligned}
$$




$$
\begin{aligned}
\varepsilon_{y x}^{\prime}= & \\
& \frac{\left(1+\frac{\partial u_{1}}{\partial x}\right)\left((z+\Delta z) a_{21}\right)}{} \\
& +\left(\frac{\partial v_{1}}{\partial x}\right)\left((z+\Delta z) a_{22}\right) \\
& \left.+\frac{\partial u_{1}}{\partial x}\right)\left((z+\Delta z) a_{11}\right) \\
& +\left(\frac{\partial v_{1}}{\partial x}\right)\left((z+\Delta z) a_{12}\right)
\end{aligned}
$$

Case 5: $\theta_{x}=0, \theta_{z}=0$

$$
\begin{aligned}
& a_{11}=\cos \theta_{y} \\
& a_{12}=0 \\
& a_{21}=0 \\
& a_{22}=1 \\
& a_{31}=-\sin \theta_{y} \\
& a_{32}=0
\end{aligned}
$$

$$
\begin{gathered}
\varepsilon_{x x}^{\prime}=z \frac{\left(1+\varepsilon_{x x}\right)\left((z+\Delta z) \cos \theta_{y}+\sin \theta_{y} O_{x}\right)}{\left(z+\Delta z-\sin \theta_{y}\left(x+u_{1}-O_{x}\right)\right)^{2}}-1 \\
\varepsilon_{y y}^{\prime}=z \frac{\left(\frac{\partial u_{1}}{\partial y}\right)\left(y+v_{1}\right)\left(\sin \theta_{y}\right)}{+\left(1+\varepsilon_{y y}\right)\left((z+\Delta z)-\left(x+u_{1}-O_{x}\right)\left(\sin \theta_{y}\right)\right)}-1 \\
\left(z+\Delta z-\left(x+u_{1}-O_{x}\right)\left(\sin \theta_{y}\right)\right)^{2}
\end{gathered}
$$




$$
\begin{aligned}
\varepsilon_{x y}^{\prime}= & \frac{\left(\frac{\partial u_{1}}{\partial y}\right)\left((z+\Delta z) \cos \theta_{y}+\left(y+v_{1}-O_{y}\right)\left(\sin \theta_{y} O_{x}\right)\right.}{\left(\frac{\partial u_{1}}{\partial y}\right)\left(\left(y+v_{1}-O_{y}\right)\left(\sin \theta_{y}\right)-\sin \theta_{y} O_{y}\right)} \\
& +\left(1+\frac{\partial v_{1}}{\partial y}\right)\left((z+\Delta z)-\left(x+u_{1}-O_{x}\right)\left(\sin \theta_{y}\right)\right) \\
\varepsilon_{y x}^{\prime}= & \left.\quad+\frac{\partial u_{1}}{\partial x}\right)\left(\left(y+v_{1}-O_{y}\right)\left(\sin \theta_{y}\right)+\sin \theta_{y} O_{y}\right) \\
& +\left(\frac{\partial v_{1}}{\partial x}\right)\left((z+\Delta z)+\left(x+u_{1}-O_{x}\right)\left(\sin \theta_{y}\right)\right) \\
& \left(1+\frac{\partial u_{1}}{\partial x}\right)\left((z+\Delta z) \cos \theta_{y}+\sin \theta_{y} O_{x}\right)
\end{aligned}
$$

Case 6: $\theta_{y}=0, \theta_{z}=0$

$$
\begin{aligned}
& a_{11}=1 \\
& a_{12}=0 \\
& a_{21}=0 \\
& a_{22}=\cos \theta_{x} \\
& a_{31}=0 \\
& a_{32}=\sin \theta_{x}
\end{aligned}
$$




$$
\begin{aligned}
& \varepsilon_{x x}^{\prime}= \\
& \left(1+\frac{\partial u_{1}}{\partial x}\right)\left((z+\Delta z)+\left(y+v_{1}-O_{y}\right)\left(a_{32}\right)\right) \\
& =z \frac{+\left(\frac{\partial v_{1}}{\partial x}\right)\left(\left(x+u_{1}-O_{x}\right)\left(-a_{32}\right)-a_{32} O_{x}\right)}{\left(z+\Delta z+\left(y+v_{1}-O_{y}\right) a_{32}\right)^{2}}-1 \\
& \varepsilon_{y y}^{\prime}= \\
& =z \frac{\left(1+\frac{\partial v_{1}}{\partial y}\right)\left((z+\Delta z) a_{22}+a_{32} O_{y}\right)}{\left(z+\Delta z+\left(y+v_{1}-O_{y}\right) a_{32}\right)^{2}}-1 \\
& \varepsilon_{x y}^{\prime}= \\
& \left(\frac{\partial u_{1}}{\partial y}\right)\left((z+\Delta z)+\left(y+v_{1}-O_{y}\right)\left(a_{32}\right)\right) \\
& +\left(1+\frac{\partial v_{1}}{\partial y}\right)\left(+\left(x+u_{1}-O_{x}\right)\left(-a_{32}\right)-a_{32} O_{x}\right) \\
& \left(1+\frac{\partial v_{1}}{\partial y}\right)\left((z+\Delta z) a_{22}+a_{32} O_{y}\right) \\
& \varepsilon_{y x}^{\prime}= \\
& \left(\frac{\partial v_{1}}{\partial x}\right)\left((z+\Delta z) a_{22}-a_{32} O_{y}\right) \\
& \left(1+\frac{\partial u_{1}}{\partial x}\right)\left((z+\Delta z) a_{11}+\left(y+v_{1}-O_{y}\right)\left(a_{11} a_{32}\right)\right) \\
& +\left(\frac{\partial v_{1}}{\partial x}\right)\left(\left(x+u_{1}-O_{x}\right)\left(-a_{11} a_{32}\right)-a_{32} O_{x}\right)
\end{aligned}
$$


Case 7: $\theta_{x}=0, \theta_{y}=0, \theta_{z}=0$

$$
\begin{aligned}
& a_{11}=1 \\
& a_{12}=0 \\
& a_{21}=0 \\
& a_{22}=1 \\
& a_{31}=0 \\
& a_{32}=0
\end{aligned}
$$

$$
\begin{aligned}
& \varepsilon_{x x}^{\prime}=z \frac{\left(1+\frac{\partial u_{1}}{\partial x}\right)}{(z+\Delta z)}-1 \\
& \varepsilon_{y y}^{\prime}=z \frac{\left(1+\frac{\partial v_{1}}{\partial y}\right)}{(z+\Delta z)}-1 \\
& \varepsilon_{x y}^{\prime}=\frac{\left(\frac{\partial u_{1}}{\partial y}\right)}{\left(1+\frac{\partial v_{1}}{\partial y}\right)}=\varepsilon_{x y} \\
& \varepsilon_{y x}^{\prime}=\frac{\left(\frac{\partial v_{1}}{\partial x}\right)}{\left(1+\frac{\partial u_{1}}{\partial x}\right)}=\varepsilon_{y x}
\end{aligned}
$$




\section{Appendix C}

\section{Error Contribution Summary}

The summary is designed to be a quick-reference guide for users of the $\mathrm{X}$-ray system during experiment to quantify anticipated DIC error based on either contrastto-noise ratio, or out-of-plane motion.

\section{C.1 Contrast-to-Noise Ratio}

The following figure shows the experimental results of contrast-to-noise ratio and DIC accuracy for artificial images with pure Poisson noise, and experimentally acquired images.

\section{C.2 Out-of-Plane Motion}

Out-of-plane motion is a known caused of error in optical DIC. A 3D analytical model was developed to quantify perceived strain caused by out of plane motion of an X-ray system. This section includes general quick-reference error plots for a range of scenarios likely to be encountered experimentally. 


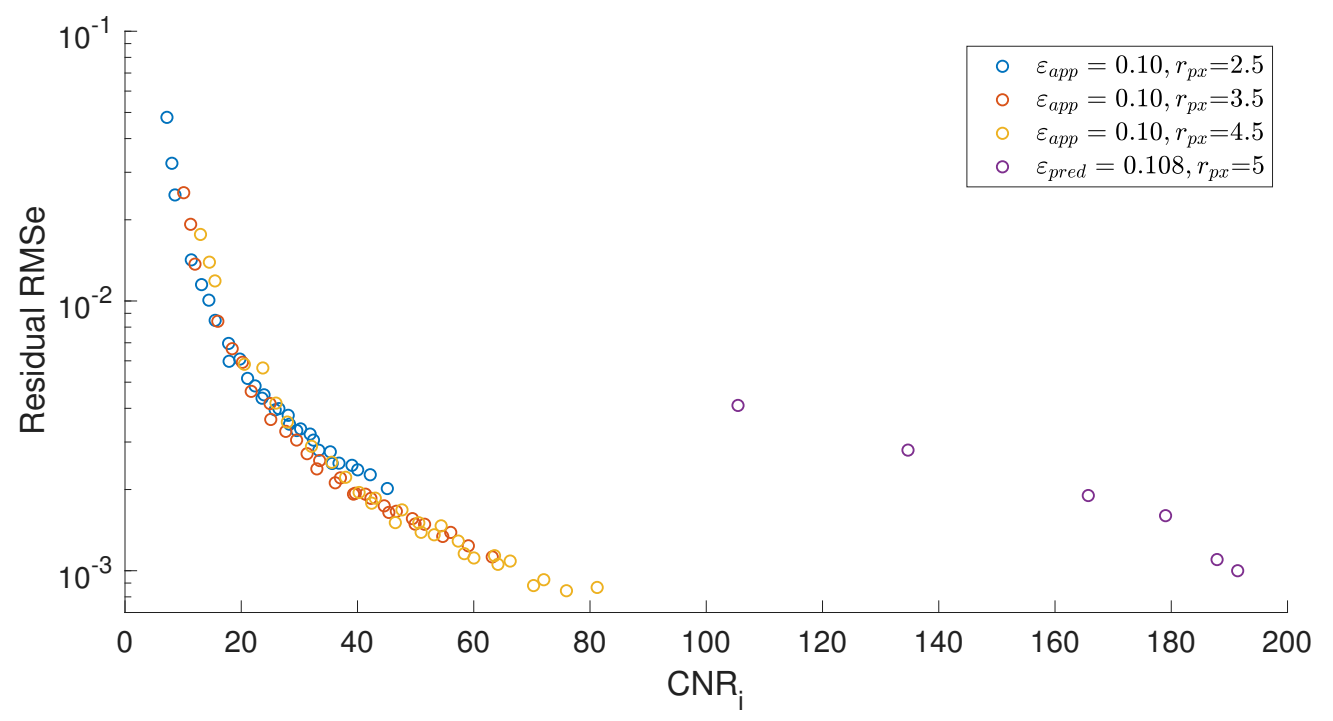

Figure C.1: Root mean squared error of residual (predicted-measured) as a function of image-level CNR for strain $(\epsilon \approx 10)$ applied to artificially generated images ( $r=2.5,3.5$, and 4.5 pixels) and experimentally produced images $(r \geq 5$ pixels).

\section{C.2.1 Out-of-Plane Translation}

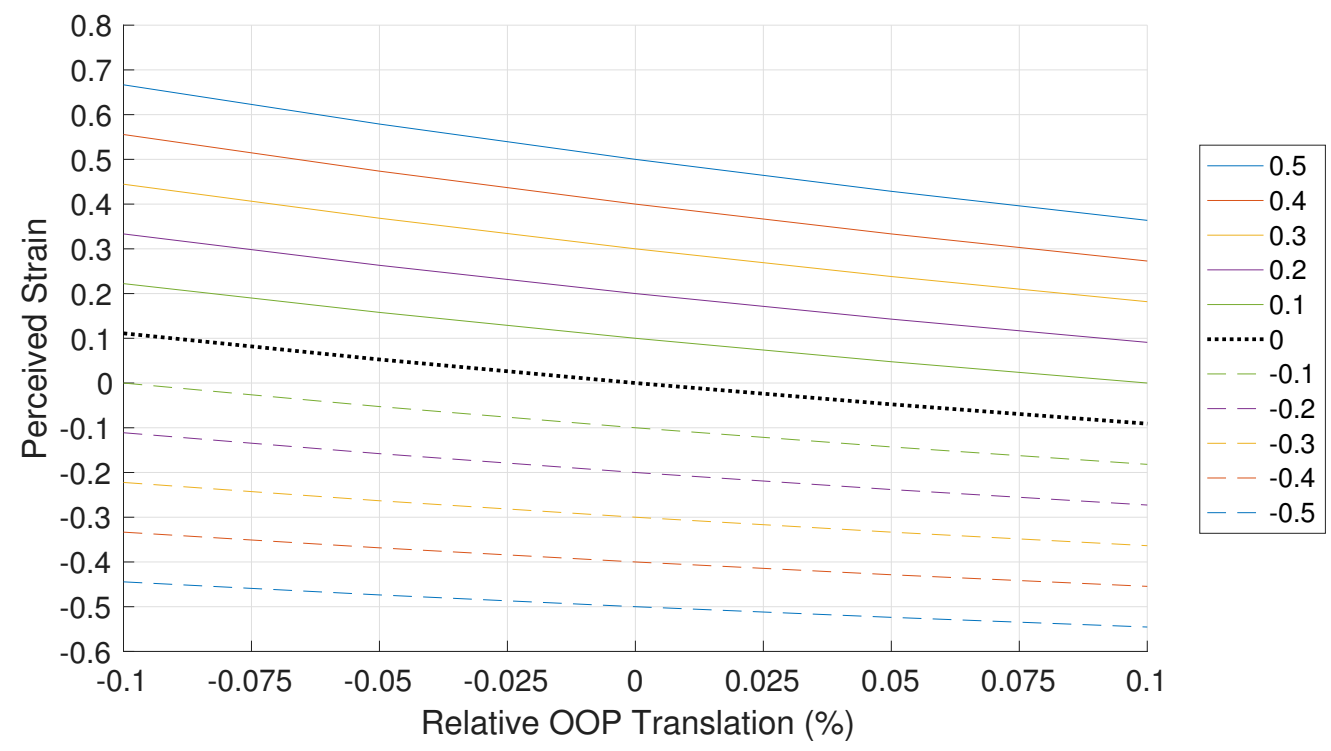

Figure C.2: Perceived normal strain versus relative out-of-plane translation for several applied strains. 


\section{C.2.2 Out-of-Plane Rotation (1 axis)}

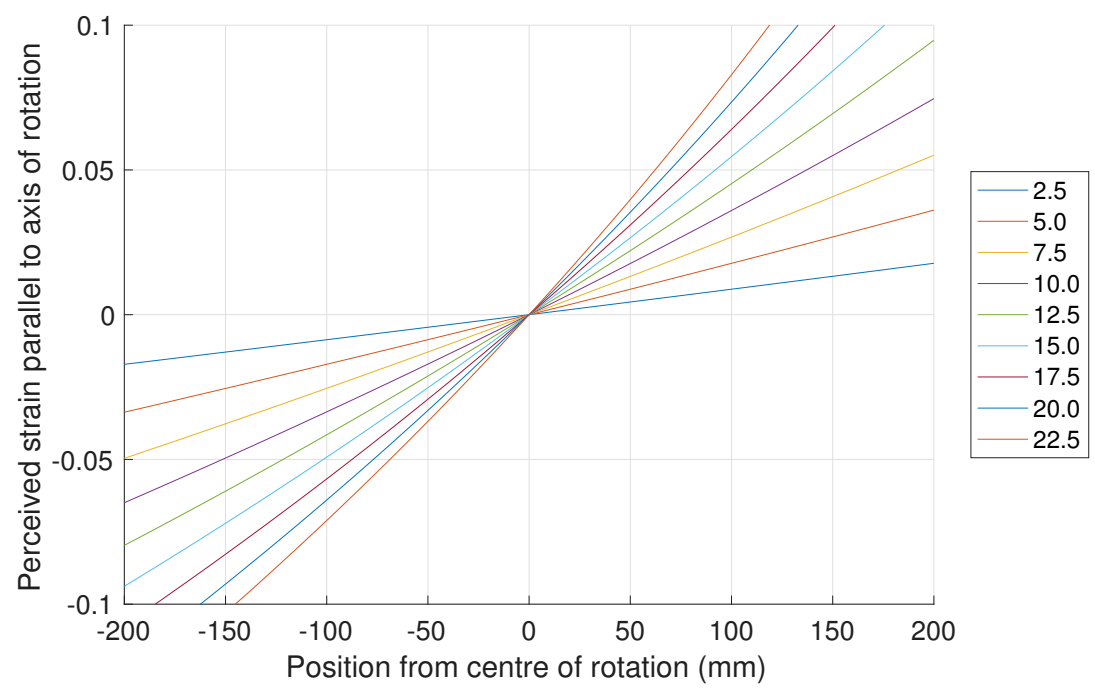

Figure C.3: Perceived normal strain parallel to the axis of rotation versus original distance from centre of rotation for several uniaxial rotation angles.

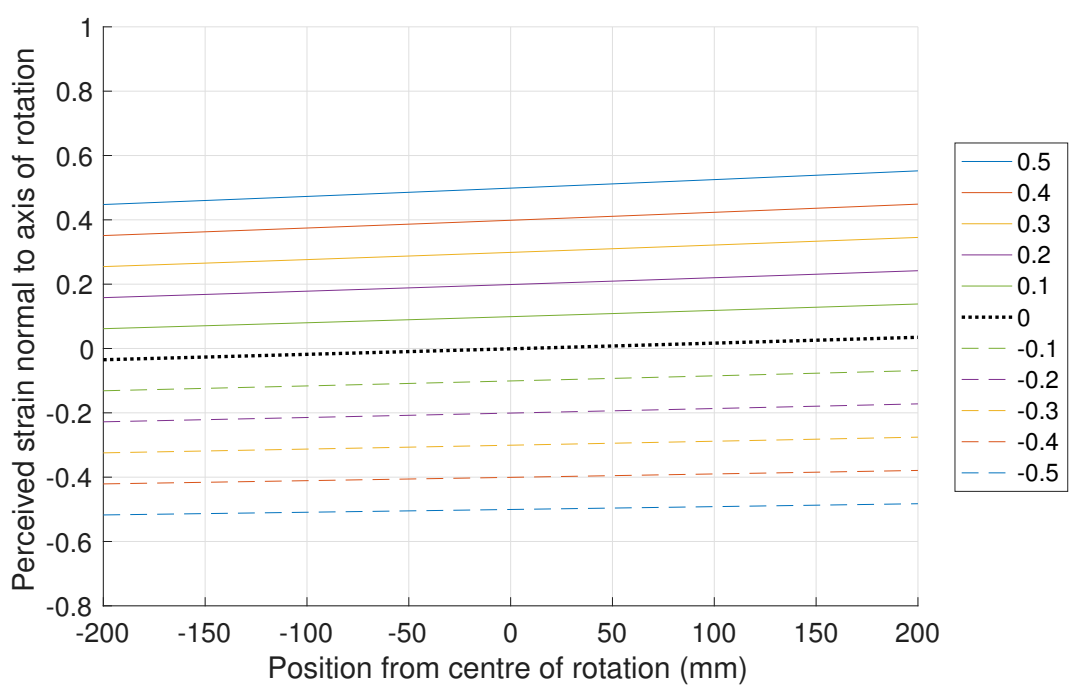

Figure C.4: Perceived normal strain perpendicular to the axis of rotation versus original distance from centre of rotation for several applied strain for uniaxial rotation of $2.5^{\circ}$. 


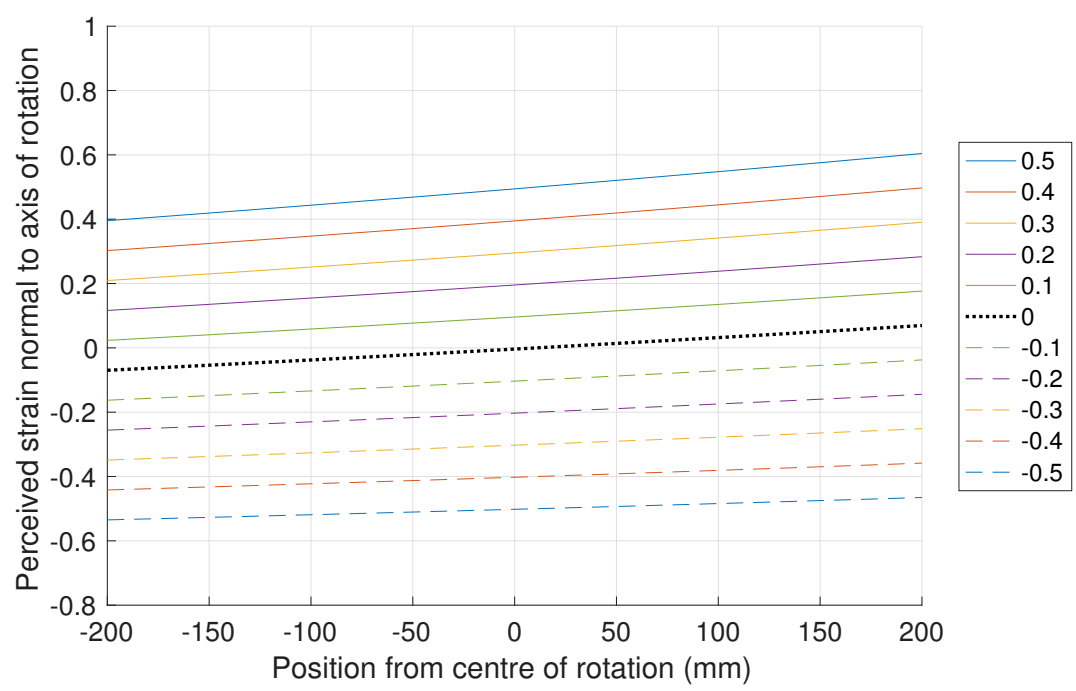

Figure C.5: Perceived normal strain perpendicular to the axis of rotation versus original distance from centre of rotation for several applied strain for uniaxial rotation of $5.0^{\circ}$.

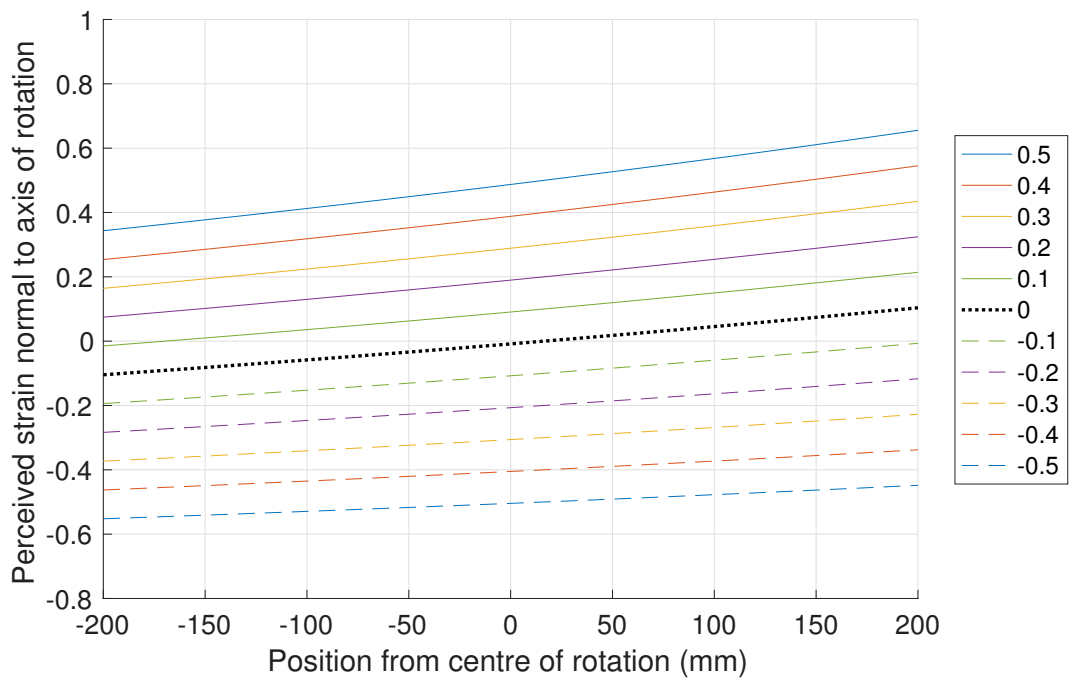

Figure C.6: Perceived normal strain perpendicular to the axis of rotation versus original distance from centre of rotation for several applied strain for uniaxial rotation of $7.5^{\circ}$. 


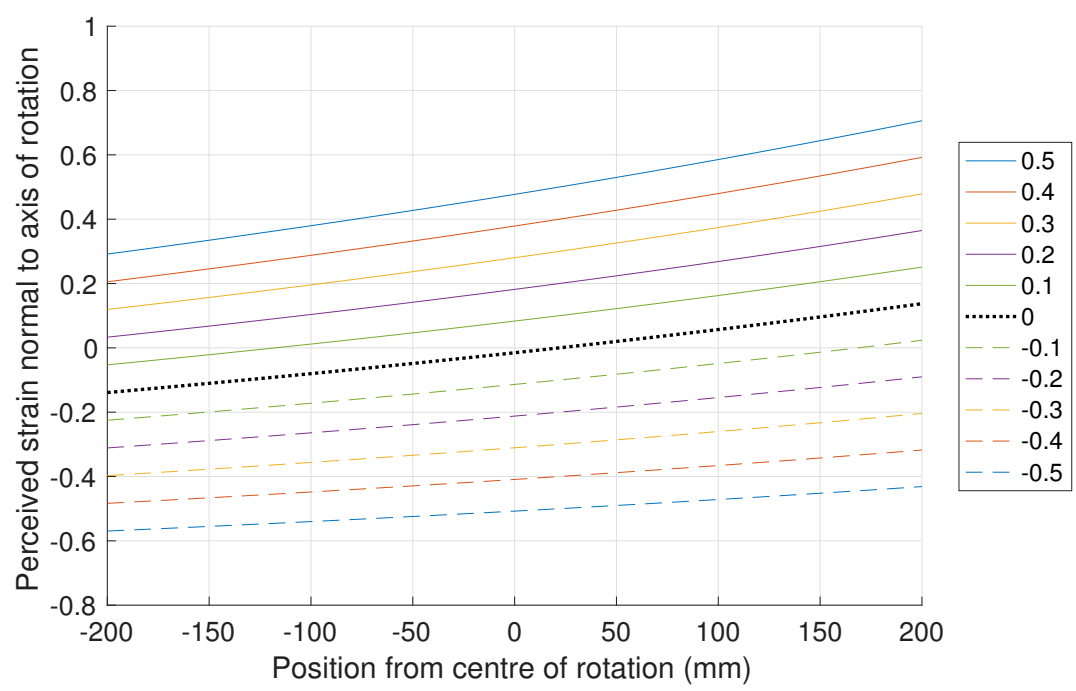

Figure C.7: Perceived normal strain perpendicular to the axis of rotation versus original distance from centre of rotation for several applied strain for uniaxial rotation of $10.0^{\circ}$.

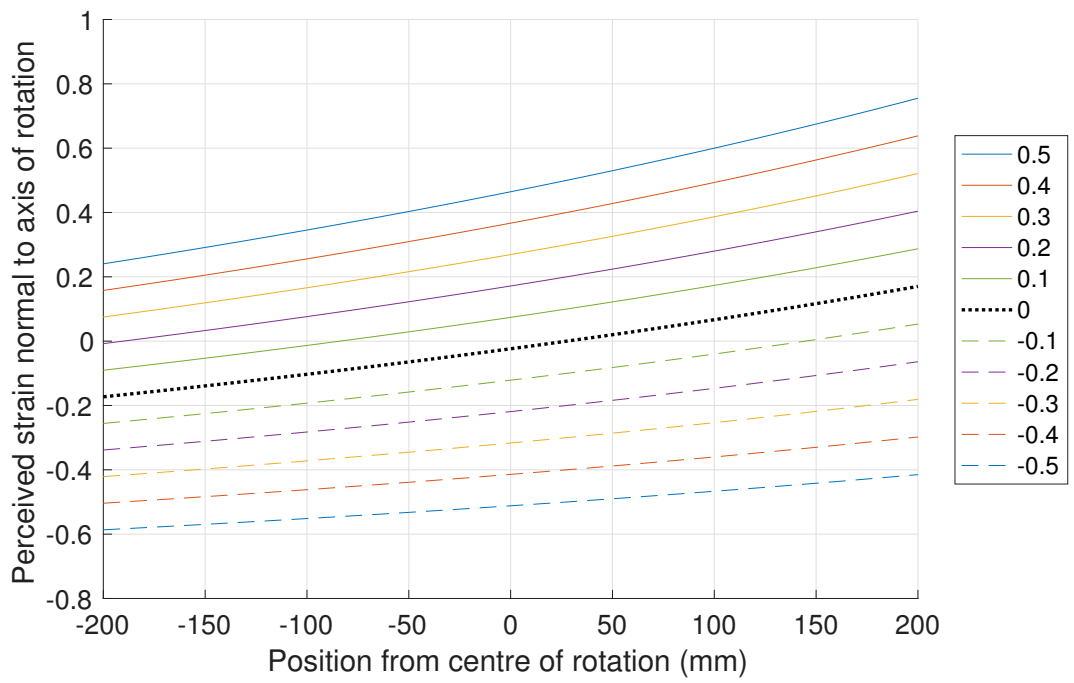

Figure C.8: Perceived normal strain perpendicular to the axis of rotation versus original distance from centre of rotation for several applied strain for uniaxial rotation of $12.5^{\circ}$. 


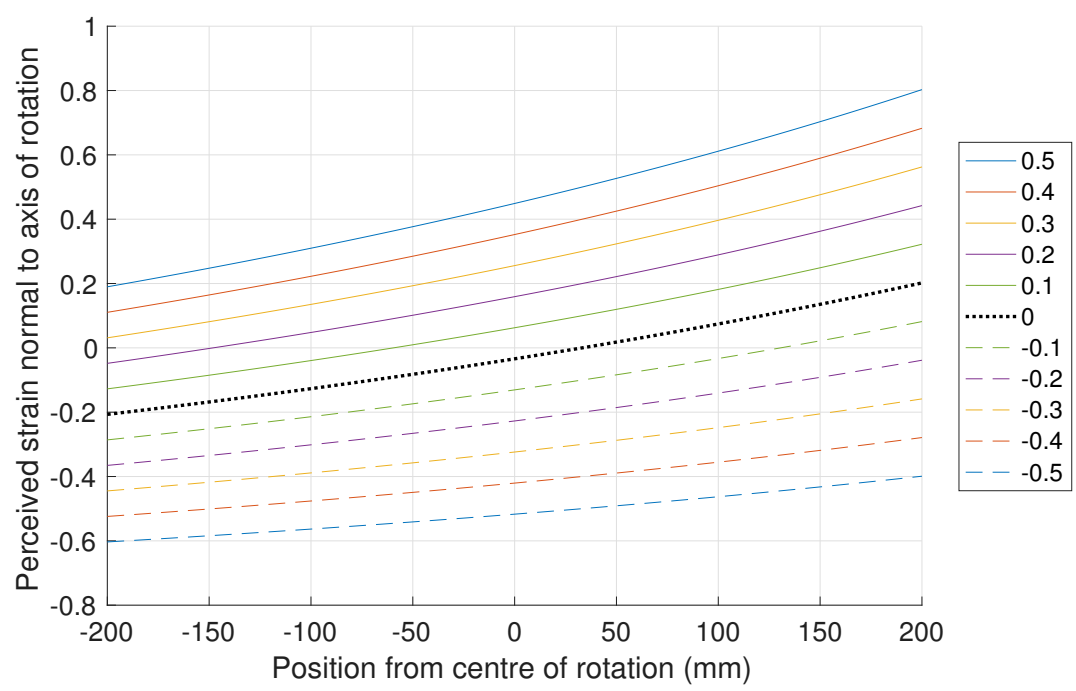

Figure C.9: Perceived normal strain perpendicular to the axis of rotation versus original distance from centre of rotation for several applied strain for uniaxial rotation of $15.0^{\circ}$.

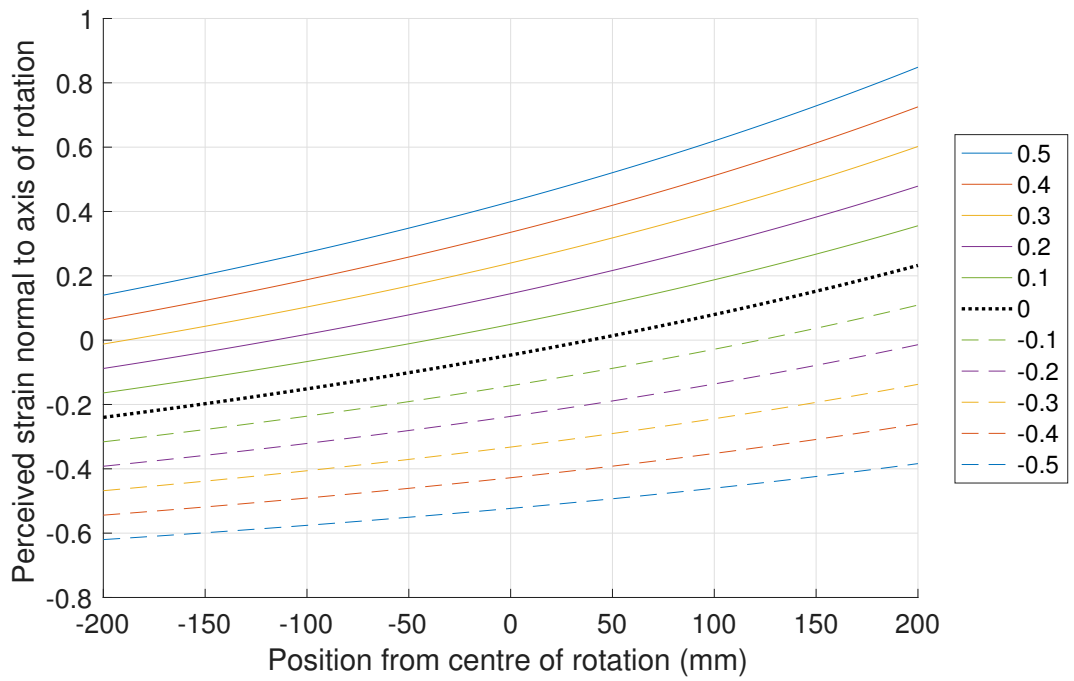

Figure C.10: Perceived normal strain perpendicular to the axis of rotation versus original distance from centre of rotation for several applied strain for uniaxial rotation of $17.5^{\circ}$. 


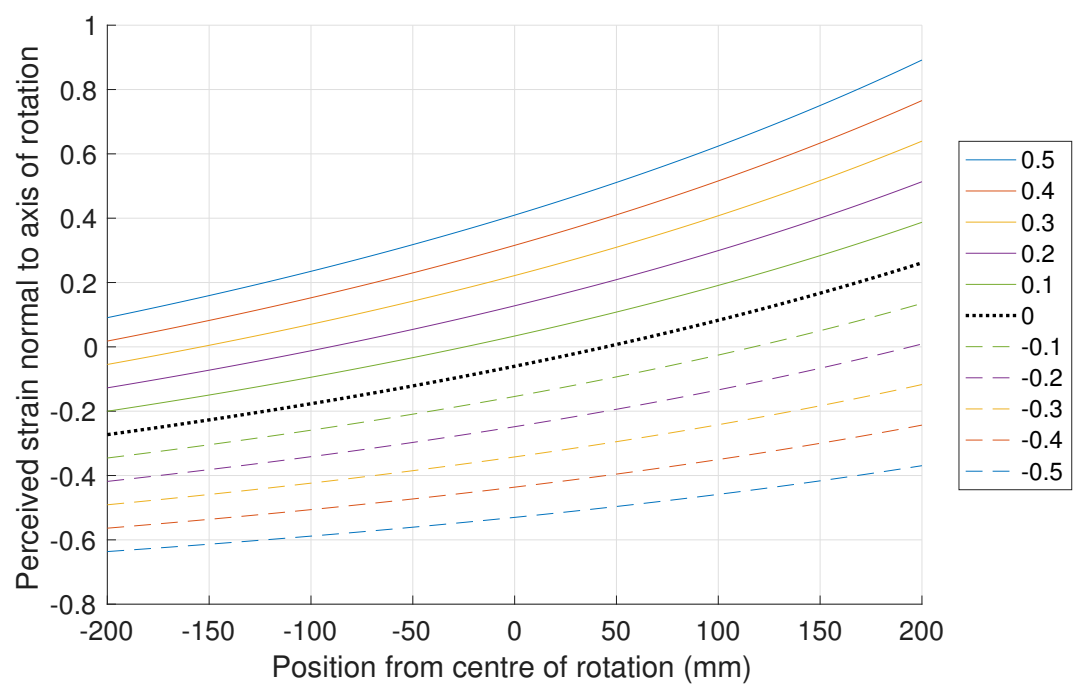

Figure C.11: Perceived normal strain perpendicular to the axis of rotation versus original distance from centre of rotation for several applied strain for uniaxial rotation of $20.0^{\circ}$.

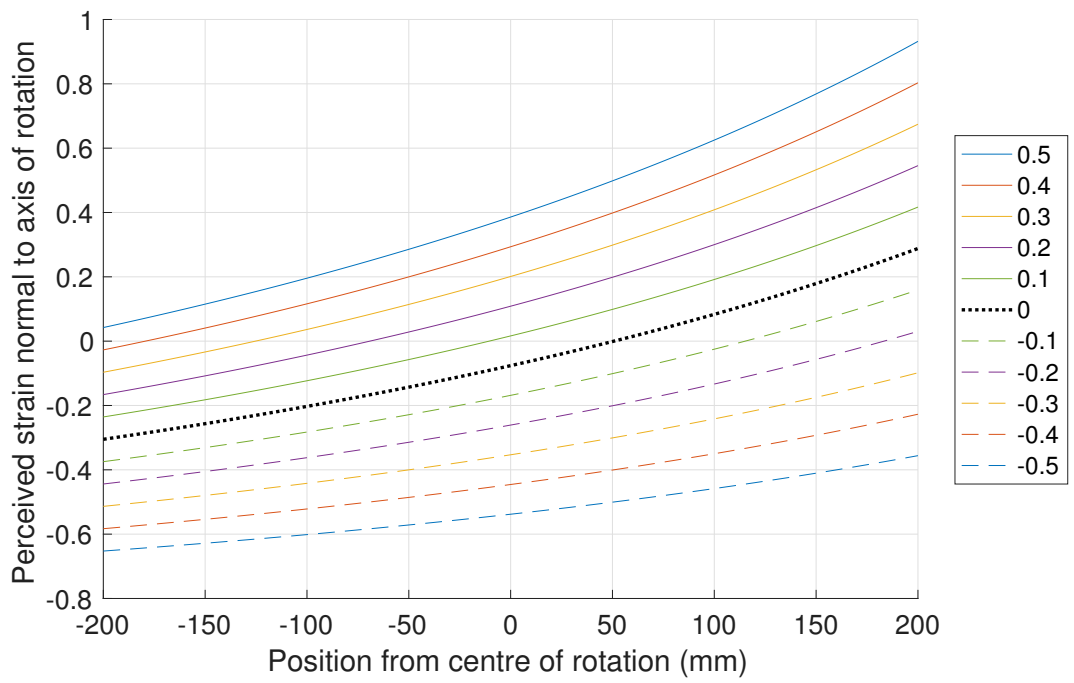

Figure C.12: Perceived normal strain perpendicular to the axis of rotation versus original distance from centre of rotation for several applied strain for uniaxial rotation of $22.5^{\circ}$. 


\section{Appendix D}

\section{Headform Displacement Plots}

Figure 77 illustrated the deformation field inside the $\mathrm{BI}^{2} \mathrm{PED}$ headform in $5 \mathrm{~ms}$ increments after a $55 \mathrm{~cm}$ drop. The images provided here, captured at $1000 \mathrm{fps}$ are in a larger format with additional frames shown.

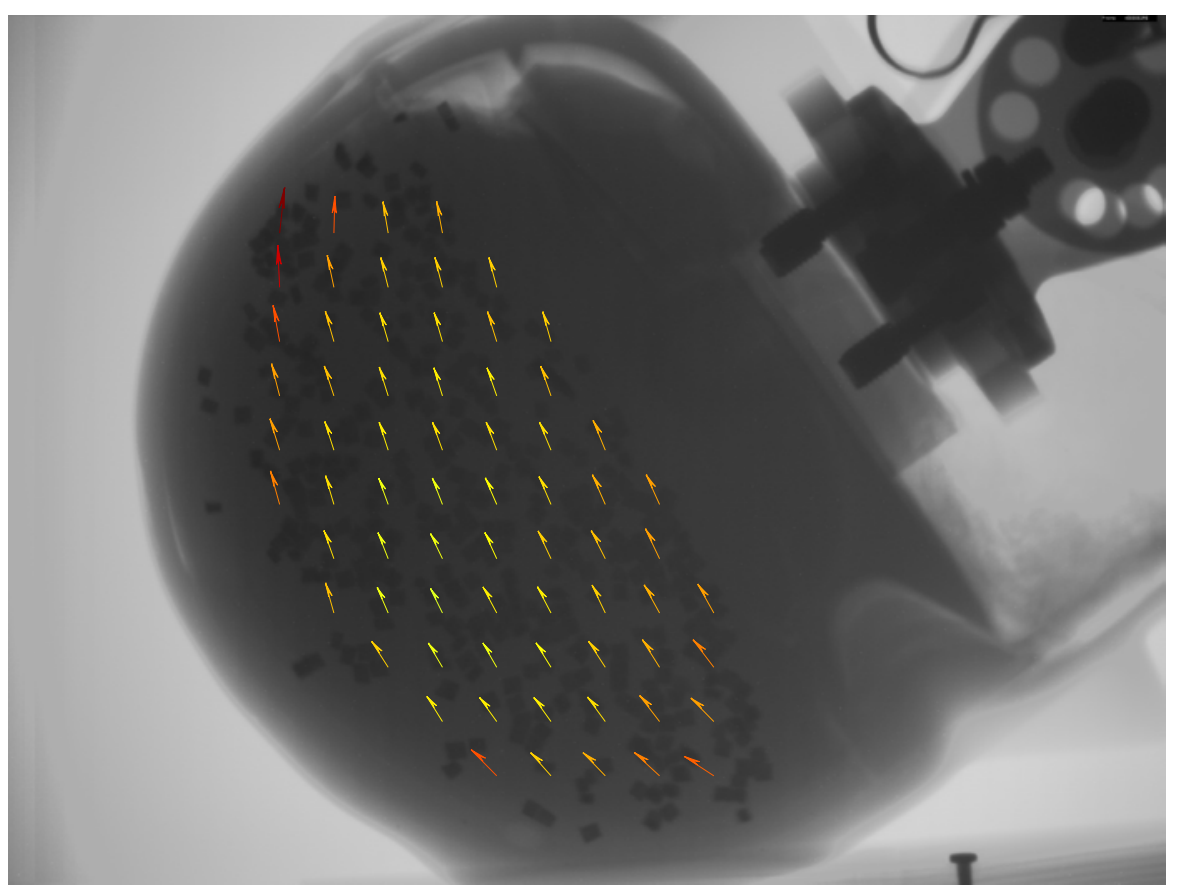

Figure D.1: $\mathrm{BI}^{2} \mathrm{PED}$ Headform: $5 \mathrm{~ms}$ post-impact 


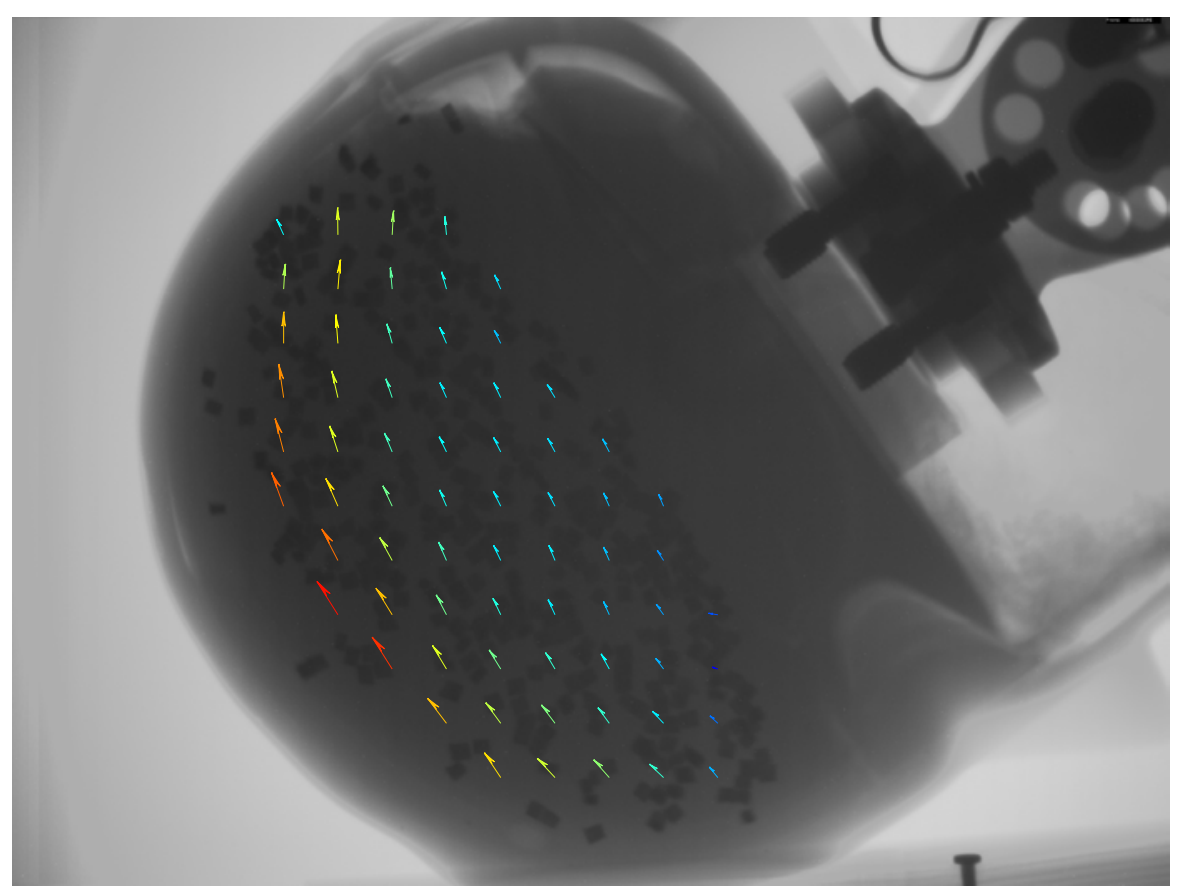

Figure D.2: $\mathrm{BI}^{2} \mathrm{PED}$ Headform: $10 \mathrm{~ms}$ post-impact

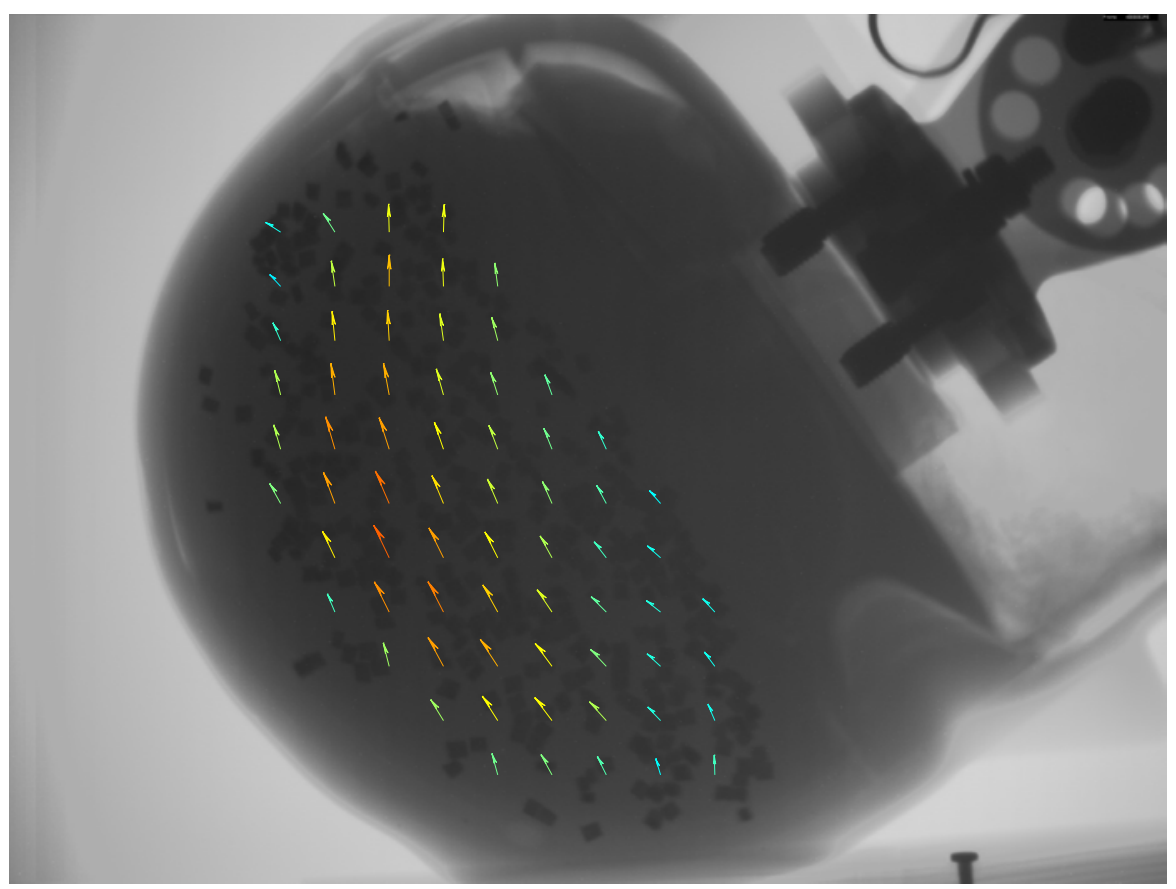

Figure D.3: $\mathrm{BI}^{2} \mathrm{PED}$ Headform: $15 \mathrm{~ms}$ post-impact 


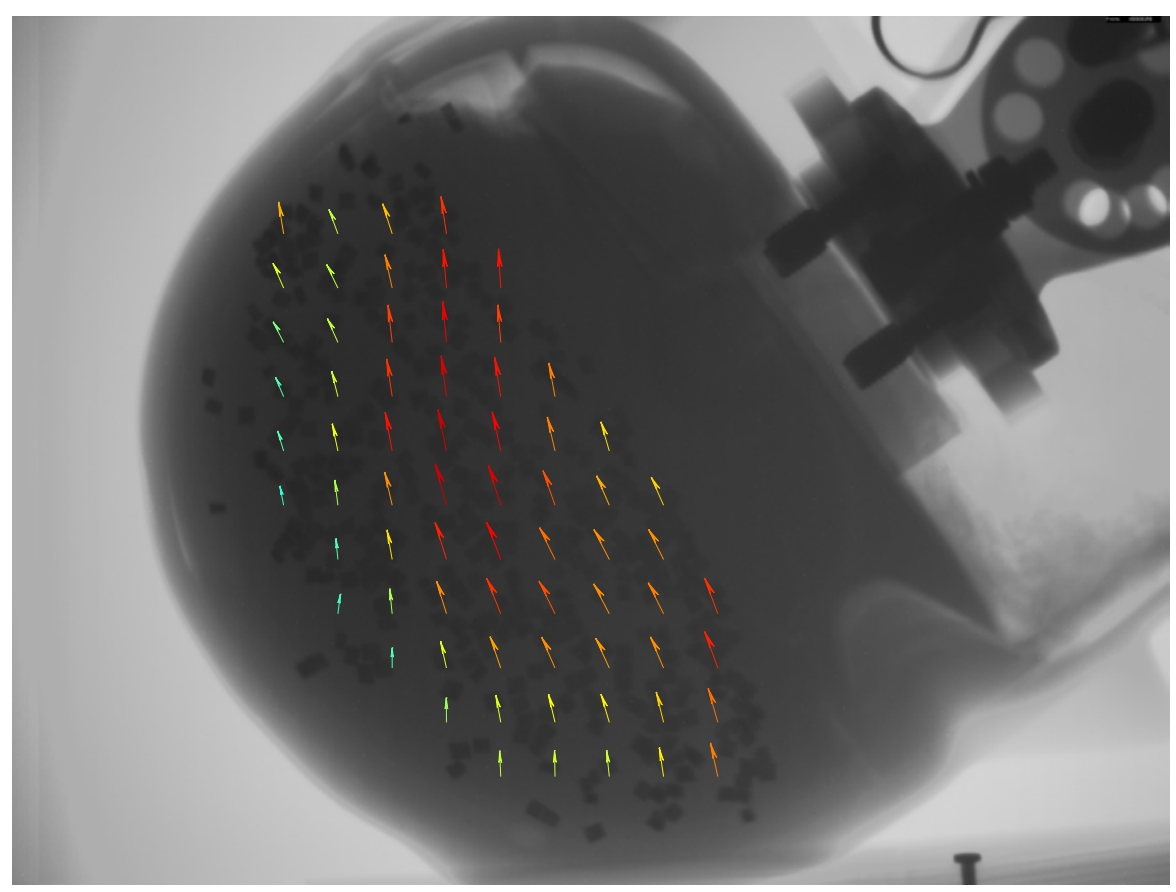

Figure D.4: $\mathrm{BI}^{2}$ PED Headform: $20 \mathrm{~ms}$ post-impact

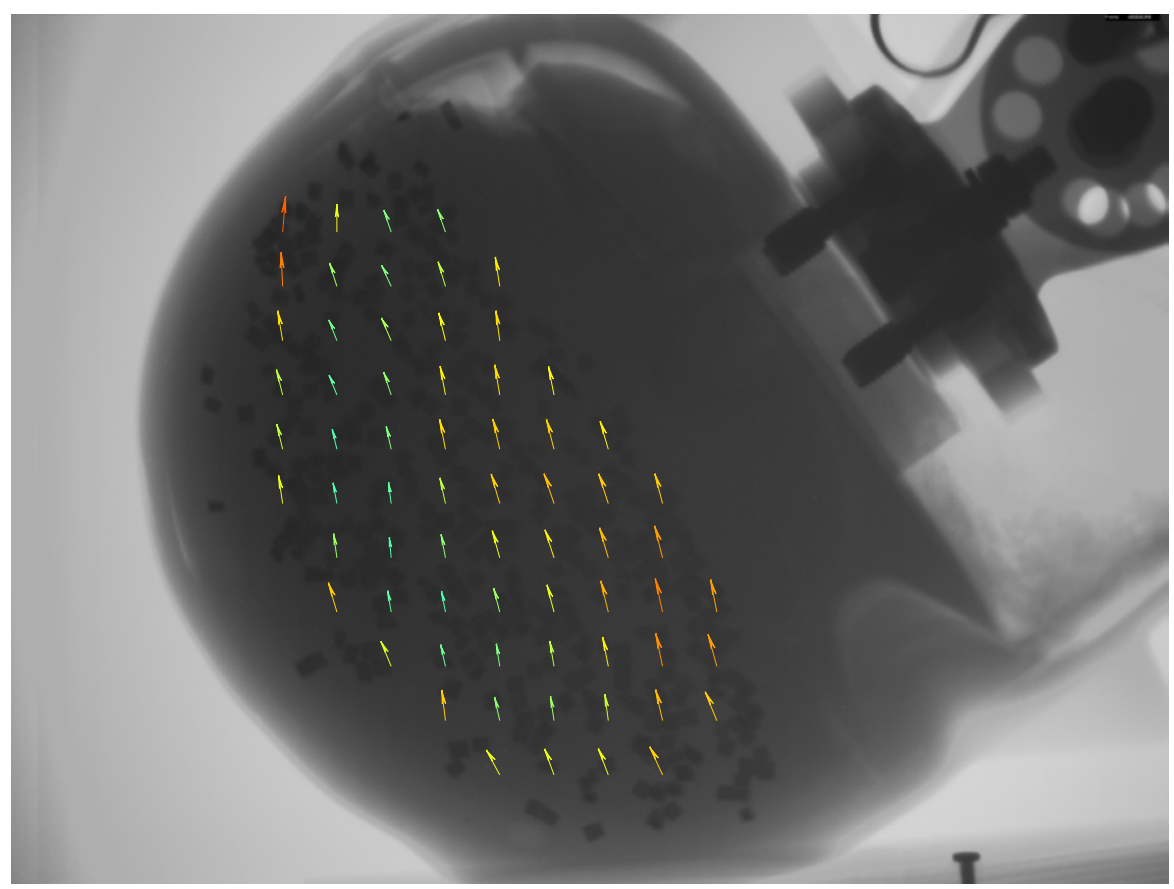

Figure D.5: $\mathrm{BI}^{2} \mathrm{PED}$ Headform: $25 \mathrm{~ms}$ post-impact 


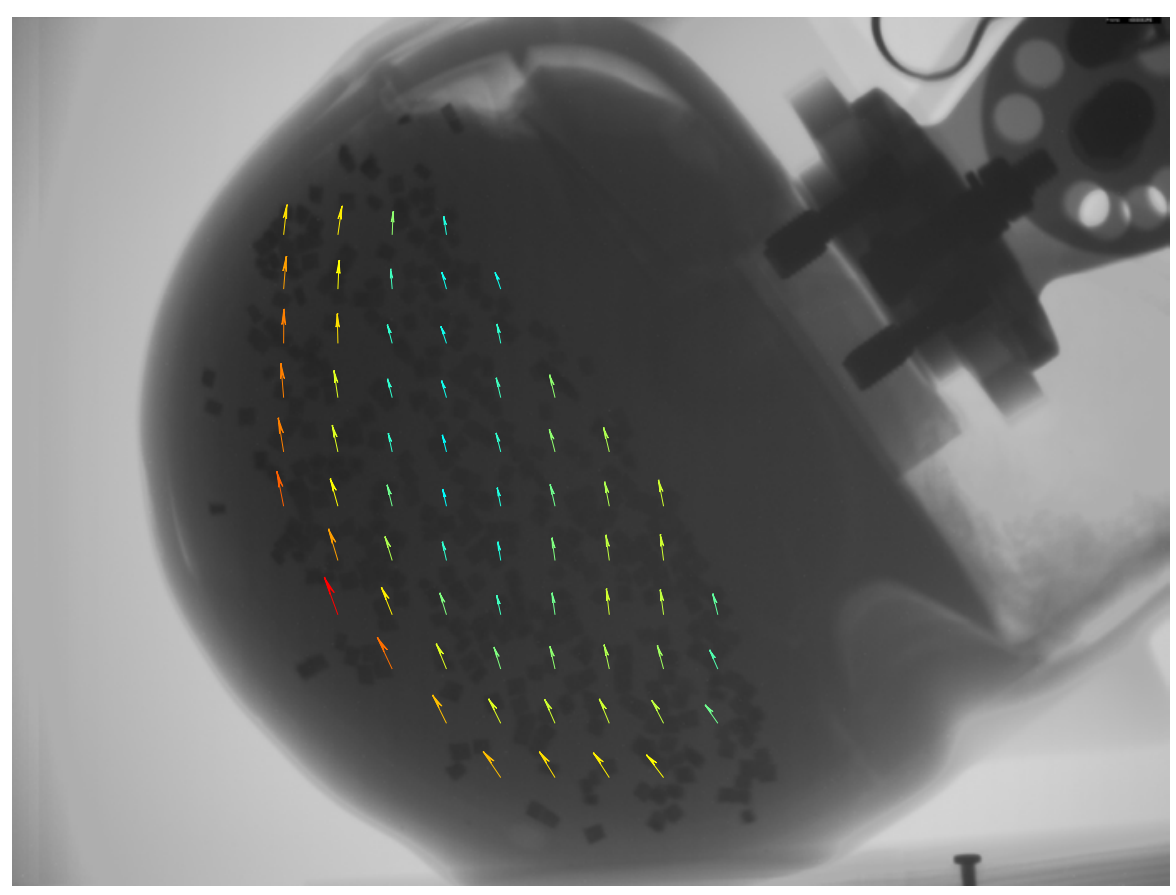

Figure D.6: $\mathrm{BI}^{2} \mathrm{PED}$ Headform: $30 \mathrm{~ms}$ post-impact

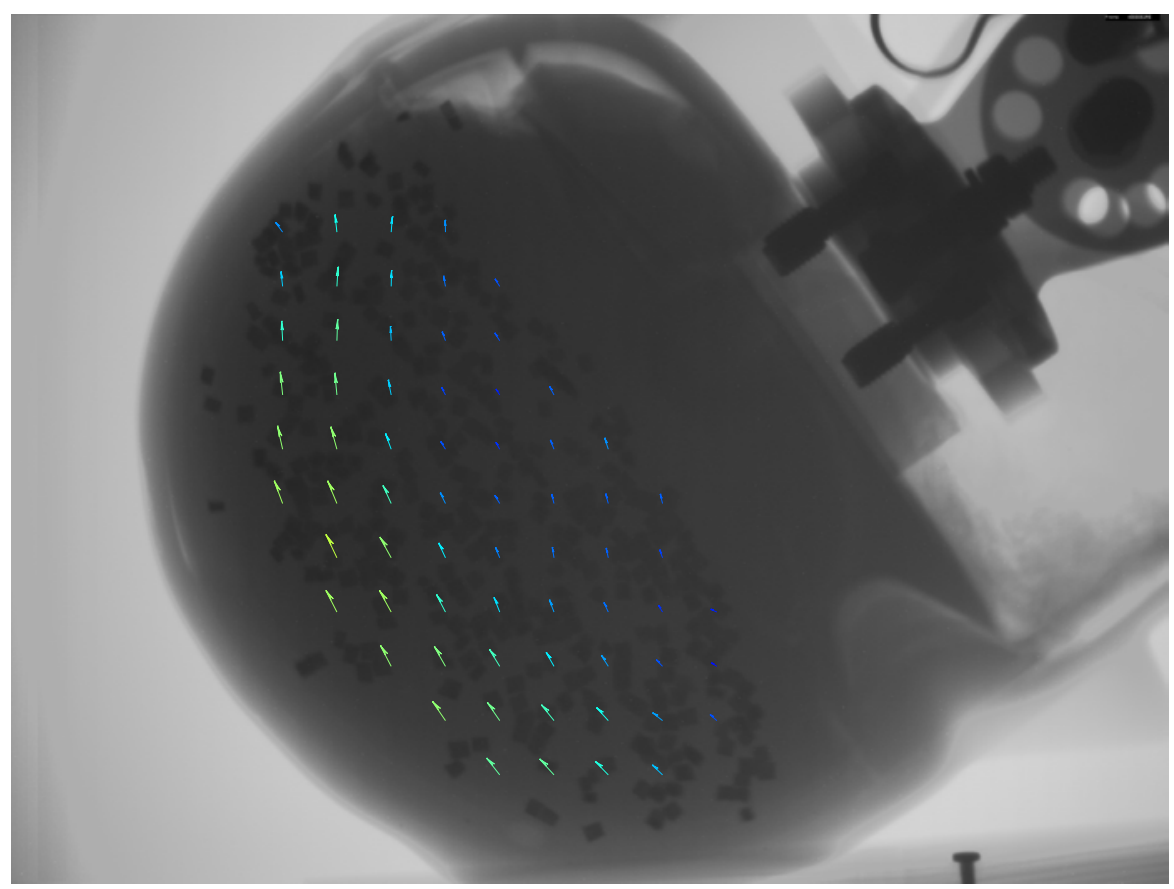

Figure D.7: $\mathrm{BI}^{2} \mathrm{PED}$ Headform: $35 \mathrm{~ms}$ post-impact 


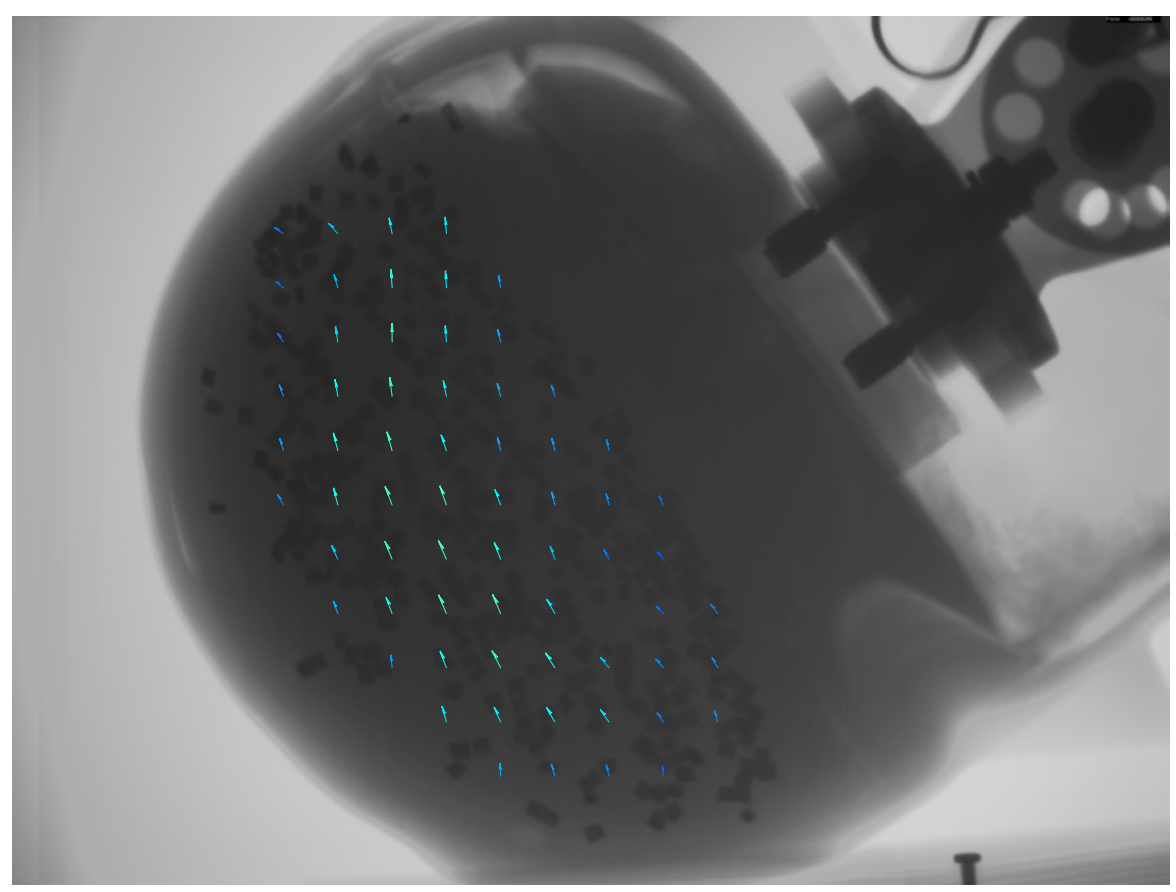

Figure D.8: $\mathrm{BI}^{2} \mathrm{PED}$ Headform: $40 \mathrm{~ms}$ post-impact

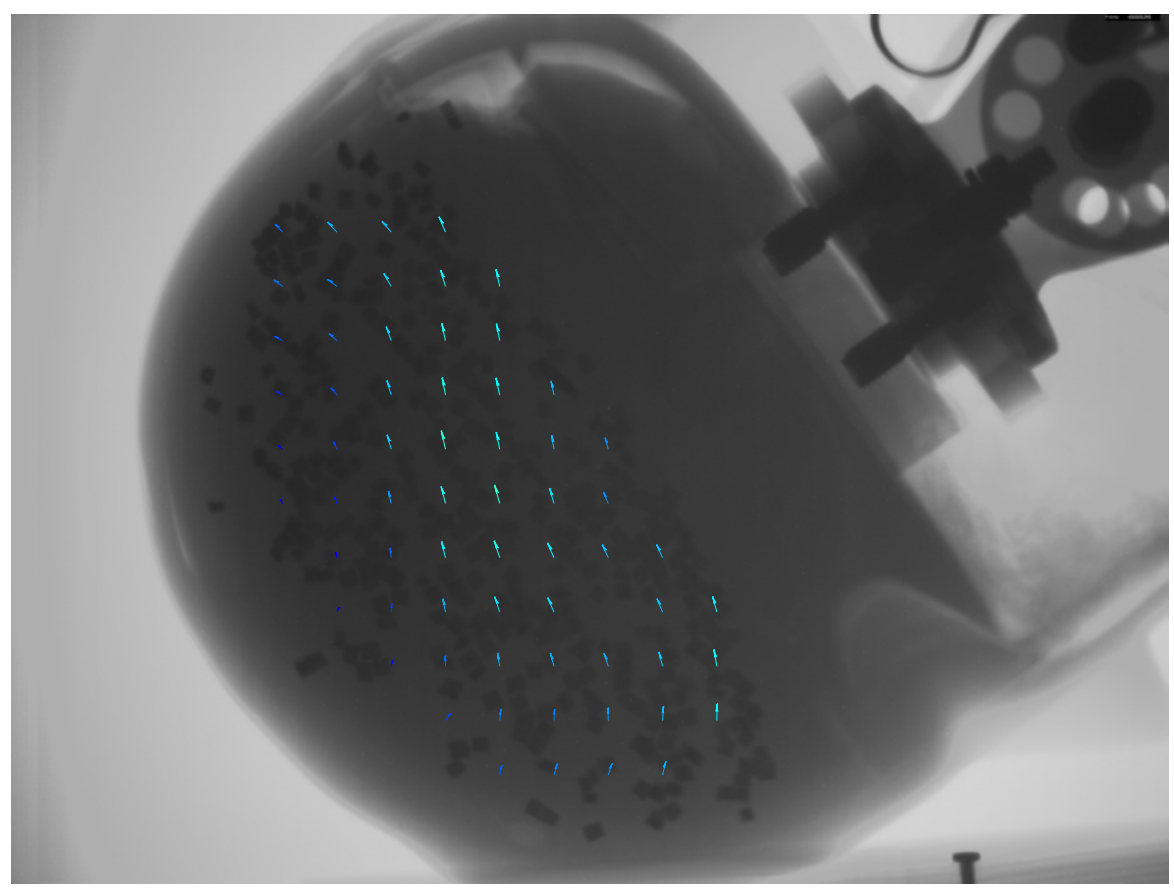

Figure D.9: $\mathrm{BI}^{2} \mathrm{PED}$ Headform: $45 \mathrm{~ms}$ post-impact 


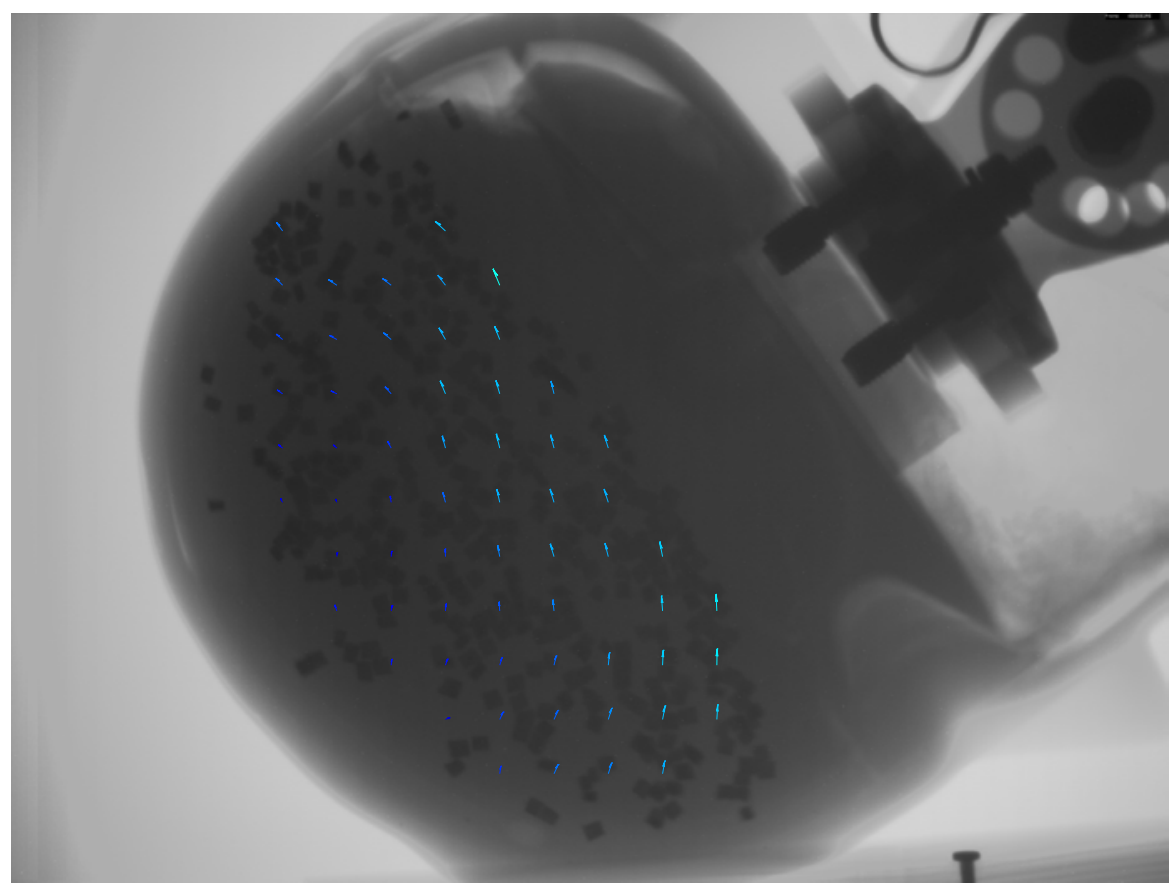

Figure D.10: $\mathrm{BI}^{2} \mathrm{PED}$ Headform: $50 \mathrm{~ms}$ post-impact 